\title{
Behavior Genetics Association 47th Annual Meeting Abstracts
}

\section{The etiology of social aggression}

Brooke Slawinski, Michigan State University, United States; Kelly Klump, Michigan State University, United States; S. Alexandra Burt, Michigan State University, United States

Social aggression (SA) is a form of antisocial behavior in which social relationships and social status are used to damage reputations and inflict emotional harm on others. Despite extensive research focused on understanding the prevalence and consequences of SA, only three studies have examined the underlying etiology of these behaviors, with markedly inconsistent results. Moreover, all of these studies relied on a classical twin design (CTD), which can result in biased heritability estimates when the strict assumptions of the design are violated. The current study sought to overcome this limitation by estimating the etiology of SA using a nuclear twin family (NTF) design, which requires far fewer assumptions. We also fit the CTD model using the same data to evaluate whether its assumptions may have biased previous heritability estimates. Maternal-report, paternalreport, and teacher-report data were collected for twin SA (N=1,030 pairs). Self-report data was collected for parental SA. The best-fitting NTF model for all informants was the ASFE model, indicating that additive genetic, sibling environmental, familial environmental, and non-shared environmental influences significantly contribute to the etiology of SA in middle childhood. Unlike the NTF model, the bestfitting CTD model varied dramatically across informants. Although the specific NTF parameter estimates varied, SA generally emerged as largely additive genetic $(\mathrm{A}=0.07-0.62)$ and sibling environmental $(\mathrm{S}$ $=0.18-0.50)$ in origin. The magnitude of $\mathrm{S}$ suggests that future research should attempt to identify specific sibling-level environmental influences that contribute to SA, such as peer groups, school experiences, parenting practices, and rearing neighborhood characteristics. Lastly, research has shown that the NTFM is superior to the CTD because it provides more nuanced and precise heritability estimates. Our results indicate that it may also be robust to informant effects and, therefore, a more valid assessment of etiology for these phenotypes in which informant biases are problematic.

\section{The origins of social conservatism: an extended twin family study using self- and peer-reports}

Edward Bell, Brescia University College, at Western University, Canada; Christian Kandler, Medical School Berlin, Germany; Rainer Riemann, Bielefeld University, Germany

It has long been recognized that social conservatism forms an important part of people's political orientations. This study examines key genetic and environmental sources of individual differences in this trait, using data taken from a German sample that included twins, their parents, and their spouses, and which incorporated both self- and peer-reports. The extended twin family design we used allowed for the examination of various aspects of social conservatism, such as: the effects of assortative mating and passive genotype-environment correlation; shared environmental influences originating from mothers only, fathers only, and both together; and non-parental environmental effects shared by twins. A comparison of self-report with peer-report findings indicated that although sex and age differences in social conservatism were comparable across the two rater perspectives, model analyses based on self-reports yielded substantially higher estimates of heritability, as well as higher levels of shared parental environmental influences, assortative mating, and genotype-environment correlation. These results, in particular the higher levels of heritability derived from self-report data, have important implications for how we understand social conservatism.

\section{Hybrid marriages and phenotypic heterosis in offspring: evidence from China}

Chen Zhu, China Agricultural University, China; Xiaohui Zhang, Nanjing University, China; Qiran Zhao, China Agricultural University, China

Qihui Chen China Agricultural University China In genetics, heterosis refers to the phenomenon that cross-breeding within species leads to offspring that are genetically fitter than their parents and exhibit improved phenotypic characteristics. Based on the theory of heterosis and existing genetic evidence, offspring of "hybrid" marriages (spouses originating from different states/provinces/countries/ 
areas), though relatively rare due to physical boundaries, may exhibit greater genetic fitness in terms of intelligence, height, or physical attractiveness ("distance-performance hypothesis"). This study explores whether heterosis is a contributing factor to offspring's educational attainment in China by applying a high-dimensional fixed effects (HDFE) modelling framework to the unique $0.1 \%$ microsample of the 2000 Chinese Population Census data $(\mathrm{N}=1,180,111)$. Concerning potential endogeneity of hybrid marriages, we conduct a series of robustness checks. Reassuringly, the estimated heterosis effect remains positive and significant among various measurements and holds after controlling for genetic heritability, environmental influences, and over a 1000 region and region $\times$ year fixed effects. Similar patterns are observed for both genders, although the effect for male offspring is generally stronger. Results are replicated when analyzing physical height using data from the China Health and Nutrition Survey. The current study sheds light on the potentially beneficial effects of interprovincial migration on population-level human capital accumulation. The implied heterosis effect could, therefore, be profound for Homo sapiens as a species from an evolutionary point of view. An additional important implication is that, besides environmental factors, the overall genetic influences of parents on offspring's performance may be further decomposed into a conventional heredity effect and a heteriosis effect that has been neglected previously.

\section{Lonely people tend to make fun of themselves}

Julie Aitken Schermer, University of Western Ontario, Canada; Rod Martin, University of Western Ontario, Canada; Philip Vernon, University of Western Ontario, Canada; Nicholas Martin, QIMR, Australia; Lucia Colodro Conde, QIMR, Australia; Michael Lynskey, King's College, United Kingdom

The present study examined the phenotypic, genetic, and environmental correlations between four humor styles (affiliative, selfenhancing, aggressive, and self-defeating) and loneliness in Australian adult twins. At the phenotypic level, the two adaptive humor style dimensions (affiliative and self-enhancing) were found to correlate negatively with loneliness and the two maladaptive humor style dimensions (aggressive and self-defeating) were found to have positive correlations with loneliness. Because both humor and loneliness were found to be heritable (ranging from $7 \%$ for loneliness to $35 \%$ for self-defeating humor style), bivariate genetic analyses were conducted. Significant genetic and unique environmental correlations were found between loneliness and humor styles with the exception of aggressive humor. These results indicate that the phenotypic association between loneliness and humor style arises from the influence of shared familial and unique environmental factors.

\section{A behavior genetic investigation of the relationship between vocational interests and measured intelligence}

Julie Aitken Schermer, University of Western Ontario, Canada; Philip Vernon, University of Western Ontario, Canada; Andrew Johnson, University of Western Ontario, Canada

Although the relationship between personality and vocational interests has received considerable attention, there has been significantly less research directed at the relationship between vocational interests and intelligence. A recent meta-analysis by Pässler, Beinicke, and Hell (2015) suggested that there is a significant phenotypic relationship between vocational interests and intelligence-and this raises the question as to whether these domains share genetic and/or environmental variance. To this end, the relationship between measured intelligence and self-report vocational interest factors was examined in an archival sample of adult twins. Participants completed selfreport vocational interest scales which fit a six factor solution. Although not large, the patterns of correlations between the intelligence scales and the vocational interest factors were meaningful. In particular, individuals with investigative interests tended to score higher on intelligence and these phenotypic correlations were also significant at the genetic level. These results suggest that there is some empirical support for a common biological antecedent that predicts both vocational interests and intelligence.

\section{Do different academic skills and IQ share the same genetic factor?}

Connie Suk-Han Ho, University of Hong Kong, Hong Kong; Jason C. -M. Lo, Chinese University of Hong Kong, Hong Kong; Mo Zheng, Chinese University, Hong Kong; Catherine Mcbride, Chinese University of Hong Kong, Hong Kong; Bonnie W. Y. Chow, City University of Hong Kong, Hong Kong; Simpson W. L. Wong, Education University of Hong Kong, Hong Kong; Mary M. Waye, Chinese University of Hong Kong, Hong Kong; Richard K. W. Choy, Chinese University of Hong Kong, Hong Kong; Richard Olson, University of Colorado Boulder, United States

Research has shown that general intelligence and academic attainment are highly heritable. One important question that remains unresolved is to what extent the same genes influence IQ and different academic skills like reading and mathematics. A total of 202 pairs of Chinese twins (with 92 pairs identical, and 110 pairs non-identical) were recruited from Hong Kong. The participants were first to fourth grade Chinese-English bilingual readers. Four measures, Chinese word reading, English word reading, Mathematics, Raven's Standard Progressive Matrices, were included in the present analyses. To adjust for age effects, the standardized residual scores regressing on age were used in the analyses. Results of univariate genetic analyses show that about one-third of the variance of Chinese word reading and mathematics were affected by genetic factors, while English word reading was more affected by common environmental factors. It was also found that the genetic correlation between Chinese and English reading was very high $(.90)$, while that between reading and mathematics were moderate (.48 to .53 ). The environmental correlation between reading and mathematics was around .35 , suggesting some shared home and school factors influencing general academic learning. We further tested two multivariate models to examine whether the three academic skills and IQ were affected by the same or different genetic factors. Results show that the best fitted model was having one common genetic factor. The present findings appear to support the generalist gene hypothesis for academic skills and general intelligence. Those who are born smarter tend to perform better academically in different school subjects. However, home and school education also plays some role on academic learning, especially on second language learning.

\section{Genetic/environmental architecture and stability of executive functions in midlife}

Daniel Gustavson, University of California, San Diego, United States; Matthew Panizzon, University of California, San Diego, United States; Carol Franz, University of California, San Diego, United States; Naomi Friedman, University of Colorado Boulder, United 
States; Chandra Reynolds, University of California, Riverside, United States; Kristen Jacobson, University of Chicago, United States; Hong Xian, St. Louis University, United States; Michael Lyons, Boston University, United States; William Kremen, University of California, San Diego, United States

Research on executive functions (EFs) has suggested that a general factor underlies performance across a number of EF tasks and domains. This Common EF factor is highly stable in adolescence through young adulthood, correlates with other cognitive abilities, and is explained largely by genetic influences. However, little is known about Common EF beyond young adulthood. We examined data from 1,464 middle-aged twins in the Vietnam Era Twin Study of Aging who completed seven neuropsychological measures of EFs at two time points (mean age 56 and 62), as well as measures of general cognitive ability and processing speed. Confirmatory factor analysis indicated that Common EF (at age 56) was moderately heritable $\left(\mathrm{a}^{2}\right.$ $=.46$ ), with additional evidence for shared environmental influences $\left(\mathrm{c}^{2}=.41\right)$ and nonshared environmental influences $\left(\mathrm{e}^{2}=.13\right)$. Common EF (at age 56) was moderately associated with higher general cognitive ability, measured both in early adulthood (age 20) and in midlife (age 56), and faster processing speed in midlife (age 56). These correlations were driven by shared genetic influences. After accounting for practice effects, there was a large mean-level performance decline in Common EF $(\mathrm{d}=-.60)$ between age 56 and 62, but individual differences in Common EF remained highly stable over time $(r=.97)$, with genetic and shared environmental influences showing perfect overlap $\left(r_{a}=1.0, r_{c}=1.0\right)$. Finally, we also identified a Working Memory Span-specific factor that captured variance in span tasks above and beyond Common EF. This span-specific factor was highly heritable at age $56\left(\mathrm{a}^{2}=.81\right)$, and also showed strong stability at the level of genetic and shared environmental influences $\left(r_{a}=1.0, r_{c}=\right.$ 1.0). These results suggest that the latent factor structure of EF is similar in midlife as in earlier ages, and that individual differences in EFs continue to show strong stability throughout midlife even as mean-level performance declines substantially.

\section{Sharply increased mortality among black females at older ages because of "genetic obesity"}

Guang Guo, University of North Carolina, Chapel Hill, United States; Hexuan Liu, University of Cincinati, United States

Longevity could be the single most important indicator of overall human health. Genetic inheritance is critically important for differences in longevity across species. Drawing on more than 10,000 genetic samples from the Health and Retirement Study, we investigate the association of "genetic obesity" (measured using polygenic scores constructed based on findings of a recent genome-wide association study) and all-cause mortality in four different racial and gender groups (white males, white females, black males, and black females) in the United States. We find that "genetic obesity" is only significantly associated with all-cause mortality among black females, but not among other three racial and gender groups. Black females who are in the category of "genetic obesity" experienced a mortality rate at ages 50 or older twice as high as those who are in the "normal" category. We found evidence that the association between "genetic obesity" and all-cause mortality is significantly greater among black females with lower education level than those with higher education level. In addition, the sharply heightened mortality among black females among the "genetically obese" tend to be in the group of those who are aged 50 and 65 .
Genetic background specific for $\operatorname{TgAb}$ discordant twins

Mikio Watanabe, Osaka University, Japan; Yoichi Takenaka, Kansai University, Japan; Chika Honda, Osaka University, Japan; Yoshinori Iwatani, Osaka University, Japan

Background: Epigenetic factors associated with the development of autoimmune diseases are unclear. Monozygotic twin pairs discordant for positive anti-thyroglobulin autoantibodies $(\mathrm{TgAb})$ are useful to examine the epigenetic factors because of their identical genetic background. To clarify the genetic background specific for $\mathrm{TgAb}$ discordant twins, we genotyped concordant and discordant twin pairs for $\mathrm{TgAb}$ positivity.

Subjects and Methods: 19 discordant and 185 concordant pairs for $\mathrm{TgAb}$ positivity were selected from the registry established by the Center for Twin Research at Osaka University. Gene polymorphisms were performed using the Omni5-4 BeadChip Kit (Illumina) to clarify genetic background.

Results: 155 polymorphisms specific for $\mathrm{TgAb}$ discordant pairs were significantly different in genotype frequencies from those of concordant pairs, and none of them was located on the HLA region of chromosome 6, which known as susceptible to autoimmune thyroid disease.

Conclusions: We found that the genetic background specific for $\mathrm{TgAb}$ discordant twins who are susceptible to epigenetic changes are different from that specific for TgAb-positive concordant twins.

(The authors are thankful to all the consultants from the Osaka Twin Research Group. This project was supported by University Grants from the Japanese Ministry of Education, Culture, Sports, Science and Technology, and by JSPS KAKENHI JP16H03261.)

\section{Sources of homophobia towards gay men: a multi-rater twin study}

Alexandra Zapko-Willmes, MSB Medical School Berlin, Germany, Christian Kandler, Medical School Berlin, Germany

The presented multi-rater twin study focused on genetic and environmental sources of individual differences in homophobia targeting gay men. We expected a substantial genetic and nonshared environmental effect on common variance across rater perspectives (hypothesis 1) and genetic influences on unique variance of selfratings (hypothesis 2). A sample of 869 mono- and dizygotic twins reared together as well as 1329 well-informed peers completed a questionnaire covering cognitive, affective, and discriminatory homophobic tendencies towards homosexual males. Both hypotheses could be confirmed. The findings will be discussed in terms of methodological consequences and socio-cultural environmental implications for homophobia.

\section{Investigating intergenerational transmission of risk in a unique Norwegian extended twin-family sample}

Eivind Ystrom, Norwegian Institute of Public Health, Norway, Fruhling Rijsdijk, King's College London, MRC Social, Genetic and Developmental Psychiatry Centre, Institute of Psychiatry, Psychology and Neuroscience, London, UK, United Kingdom; Line C. Gjerde, Norwegian Institute of Public Health, Norway; Espen Eilertsen, Norwegian Institute of Public Health, Norway; Tom McAdams, King's College London, United Kingdom 
Genetically informative intergenerational studies can disclose modes of transmission of risk from parent to offspring. However, such studies have either relied on moderate samples where the offspring are distributed across a wide range of developmental stages. We will apply the extended children-of-twin design to the Norwegian Mother and Child Birth Cohort ( $n=90,000$ families) where the offspring are assessed at several times at the same biological age. We will elaborate on extensions of the children-of-twin design to estimate both passive, active, and direct modes of transmission, and present examples using phenotypes such as ADHD, depression, substance use, and externalizing/internalizing behavior problems.

\section{Multivariate genetic architecture of DSM-5 alcohol use disorder}

Rohan Palmer, Emory University, United States; Leslie Brick, Brown University, United States; Arpana Agrawal, Washington University, St. Louis, United States; Matthew Keller, University of Colorado Boulder, United States; Andrew Heath, Washington University, St. Louis, United States; John Mcgeary, Brown University, United States; Laura Bierut, Washington University, St. Louis, United States; Valerie Knopik, Brown University, United States

Purpose: GWAS on alcoholism are challenged by the fact that it is a multifactorial disorder influenced by multiple interacting genes, each with small effect. Recent studies (Kendler, Aggen, Prescott, Crabbe, \& Neale, 2012; Palmer et al., 2015) suggest shared genetic influences across DSM-IV alcohol dependence (AD) symptoms, but these have been limited to DSM-IV criteria. We recently showed (Palmer et al., 2015) largely overlapping additive genetic effects using common autosomal markers providing support for the assumption of genetic homogeneity for DSM-IV indicators. The present study examined these same hypotheses in the recently expanded definition of DSM-5 alcohol use disorder (AUD) that incorporates new and additional domains of the disease once thought to be indicative of separate disorders.

Methods: Data from 2596 alcohol using individuals of European ancestry from the Study of Addiction: Genetics and Environment were used to examine the genomewide SNP-heritability $\left(\mathrm{h}^{2}{ }_{\mathrm{SNP}}\right)$ and SNP-covariance $\left(\mathrm{r}_{\mathrm{G}-\mathrm{SNP}}\right)$ between 11 DSM-5 AUD criteria. Phenotypic relationships between criteria were also examined to confirm an underlying liability of AUD. Likewise, parallel analysis of the observed genetic variance/covariance provided evidence of genetic homogeneity.

Results: Additive genetic effects on DSM-5 AUD criteria vary from mild (0.10) to moderate $(0.37)$ and largely overlap $\left(\mathrm{r}_{\mathrm{G}-\mathrm{SNP}}\right.$ range from 0.49 to 0.92 ). Additive genetic influences on the DSM-5 AUD factor was (0.36) and 0.23 on DSM-5 AUD symptom count (0.23).

Conclusion: Common genetic variants influence DSM-5 AUD criteria. Despite evidence for a common AUD factor, the evidence of only partially overlapping genetic effects across AUD criteria further substantiates the need to simultaneously model common and specific genetic effects across AUD criteria in molecular genetic studies of AUD in order to best index the underlying biology.

\section{Visualizing the nature and nurture of reading comprehension: geocoding a large diverse twin sample}

Sara Hart, Florida State University, United States; Florina Erbeli, Florida State University, United States; Oliver Davis, University of Bristol, United Kingdom; Jeanette Taylor, Florida State University, United States
Purpose: In 2012 an influential publication used spatial analysis in the UK twin project TEDS to visualize the genetic and environmental influences on various childhood phenotypes based on geographical location (Davis et al., 2012). Also, a growing body of literature indicates that socioeconomic status moderates the genetic influences on academic achievement, especially in the US (Tucker-Drob, Bates, 2016). With this paper, we used spatial analysis and visualization to map the genetic and environmental influences on reading comprehension based on household geographical location in a twin project based in the US, where great economic inequality is seen. Furthermore, we mapped neighborhood income as a potential environmental moderator.

Methods: The sample includes 2141 pairs of twins drawn from the Florida Twin Project on Reading, a sample that reflects the diverse population living across Florida. Reading comprehension was measured by the Florida Comprehension Assessment Test, and household income was be measured by US Census track median income.

Results and Discussion: An environmental hotspot was seen in the Miami area, in that environmental variation was greater on reading comprehension in this region. In line with this, there was greater variance in neighborhood income in Miami. This suggests that neighborhood income is a moderator of reading comprehension based on geographic location. A second result was that less environmental variance was seen on reading comprehension in a band across the middle of the state (the "I-4 Corridor", including Orlando). This was not reflected in differences in income variance, suggesting other environmental influences were serving as moderators in this region.

\section{References}

Davis, O.S., Haworth, C.M.A., Lewis, C.M., \& Plomin, R. (2012). Visual analysis of geocoded twin data puts nature and nurture on the map. Molecular Psychiatry, 17(9), 867-874.

Tucker-Drob, E.M., Bates, T.C. (2016). Large cross-national differences in gene $\times$ socioeconomic status interaction on intelligence. Psychological Science, 27(2), 138-149.

\section{Temporal stability in group favoritism is mostly attributable to genetic factors}

Gary Lewis, Royal Holloway, University of London, United Kingdom; Tim Bates, University of Edinburgh, United Kingdom

Twin studies of in-group favoritism have reported roughly equal influences of genetic and environmental factors. All, however, have solely relied on cross-sectional approaches, leaving open the question of whether genetic and environmental factors have similar roles on stability and change for in-group favoritism across time. While in-group favoritism is commonly perceived to reflect environmental influences, stable environmental effects are rare for psychological traits, thus suggesting that genetic influences may play the majority role in the stability of favoritism. Here we used addressed this issue using a 10-year (two-wave) longitudinal twin design. In-group favoritism showed high rank-order stability $(\mathrm{r}$ $=.67$ ). Seventy-four percent of this rank-order stability was attributable to genes. A broadly similar pattern was observed for race, ethnic, and religious favoritism. By contrast, changes in favoritism almost entirely reflected nonshared-environmental influences. These findings indicate that environmental influences underpin change in favoritism, while the stability of favoritism mostly reflects genetic influences. 
The genetic aetiology of subjective wellbeing and related positive traits: insights from diverse phenotyping

Robyn Wootton, University of Bristol, United Kingdom; Oliver Davis, University of Bristol, United Kingdom; Claire Haworth, University of Bristol, United Kingdom

The largest genome-wide association study of subjective wellbeing comprised almost 300,000 individuals and identified three genomewide significant hits (Okbay et al., 2016). To maximise sample size, cohorts were included with only single item measures of subjective wellbeing. Future directions encourage the use of more comprehensive measures of subjective wellbeing and related positive traits. Using the Twins Early Development Study (TEDS) and the Avon Longitudinal Study of Parents and Children (ALSPAC), we investigated three different measures of subjective wellbeing and other positive traits including: optimism, basic psychological needs, gratitude and meaning in life. We focus specifically on adolescents and young adults with data collected at 16 and 23 years in TEDS and ALSPAC respectively. Twin and SNP-based heritability estimates of these measures were calculated and compared suggesting moderate contribution of common genetic variants across all phenotypes. We ran a proxy-phenotype analysis using the GWAS SNPs aiming to identify novel SNPs associated with the related positive traits. Finally, we calculated polygenic scores from the GWAS data to explain the variance in our measures of subjective wellbeing and related traits in adolescence. These applications of the GWAS results indicate unique genetic components of the diverse measures and emphasise the requirement for larger datasets of positive traits to fully understand the genetic aetiology of subjective wellbeing.

\section{Predicting attention-deficit/hyperactivity disorder severity from psychosocial stress and stress response genes: a random forest regression approach}

\section{Dennis van der Meer, University of Oslo/NORMENT, Norway}

Identifying genetic variants contributing to attention-deficit/hyperactivity disorder (ADHD) is complicated by the involvement of numerous common genetic variants with small effects, interacting with each other as well as with environmental factors, such as stress exposure. Random forest regression is well-suited to explore this complexity, as it allows for the analysis of many predictors simultaneously, taking into account any higher-order interactions among them. Using random forest regression, we predicted ADHD severity, measured by Conners' Parent Rating Scales, from 686 adolescents and young adults (of which 281 were diagnosed with ADHD). The analysis included 17,374 single nucleotide polymorphisms (SNPs) across 29 genes previously linked to hypothalamic-pituitary-adrenal (HPA) axis activity, together with information on exposure to 24 individual long-term difficulties or stressful life events. The model explained $12.5 \%$ of variance in ADHD severity. The most important SNP for prediction, which also showed the est interaction with stress exposure, was located in a region regulating the expression of telomerase reverse transcriptase (TERT). Other high-ranking SNPs were found in or near NPSR1, ESR1, GABRA6, PER3, NR3C2, and DRD4. Chronic stressors were more influential than single, severe, life events. We conclude that random forest regression may be used to investigate how multiple genetic and environmental factors jointly contribute to ADHD. It is able to implicate novel SNPs of interest, interacting with stress exposure, and may explain inconsistent findings in ADHD genetics. This exploratory approach may be best combined with more hypothesis-driven research; top predictors, and their interactions with one another, should be replicated in independent samples.

\section{The influence of neurodevelopmental problems on the risk of sexual victimisation}

Agnes Ohlsson Gotby, Karolinska Institutet, Sweden; Niklas Långström, Karolinska Institutet, Sweden; Paul Lichtenstein, Karolinska Institutet, Sweden; Erik Pettersson, Karolinska Institutet, Sweden

Background: Autism spectrum disorders (ASD), Attention-deficit/ Hyperactivity disorder (ADHD), and other related neurodevelopmental problems (NDPs) might be potential risk factors for being sexually victimized, but few studies has considered comorbidity of other NDPs or the potential mechanism behind association.

Methods: Using a genetically informative prospective design, we examined the association between ASD- and ADHD-symptoms in childhood and sexual victimization in adolescence, and the relative contributions of genetic and environmental factors to this association. Parent-reports of NDPs were attained at age 9/12 years and selfreported sexual victimization at age 18 for 5,597 children participating in The Child and Adolescent Twin Study in Sweden (CATSS). A bifactor model was used to control for NDP comorbidity. To analyze genetic and environmental contributions to observed associations, we applied a Cholesky decomposition.

Results: Parent-reported ASD-, inattention and hyperactivity symptoms in childhood significantly predicted self-reported sexual victimization in adolescence in both boys and girls. However, when controlling for overall NDP symptom load (operationalized as a general NDP factor), there were no unique effects of ASD-, impulsivity-, or inattention symptoms. A Cholesky decomposition revealed that the association between the NDP general factor and sexual victimization was due to shared genetics.

Conclusions: General NDP-symptom load, rather than specific ASDor ADHD-traits, seems to be a risk factor for sexual victimization.

\section{Mediation and modification of genetic susceptibility to obesity by eating behaviours: the EDEN and Fenland cohort studies}

Blandine De Lauzon-Guillain*, INSERM, France; Emma Clifton, University of Cambridge, United Kingdom; Felix Day, University of Cambridge, United Kingdom; Karine Clement, Institute of Cardiometabolism and Nutrition, France; Soren Brage, University of Cambridge, United Kingdom; Nita Forouhi, University of Cambridge, United Kingdom; Simon Griffin, University of Cambridge, United Kingdom; Yves Akoli Koudou, INSERM, France; Veronique Pelloux-gervais, Institute of Cardiometabolism and Nutrition, France; Nick Wareham, University of Cambridge, United Kingdom; Marie-aline Charles, INSERM, France; Barbara Heude, INSERM, France; Ken Ong, University of Cambridge, United Kingdom

Background: Many genetic variants show highly robust associations with BMI. However, the mechanisms through which genetic susceptibility to obesity operate are not well understood. Potentially modifiable mechanisms, including eating behaviours, are of particular interest to public health. Here we explore whether eating behaviours mediate or modify genetic susceptibility to obesity. 
Methods: Genetic risk scores for BMI (BMI-GRS) were calculated for 3515 and 2154 adults in the Fenland and EDEN population-based cohort studies, respectively. The eating behaviours: emotional eating, uncontrolled eating and cognitive restraint, were measured using a validated questionnaire in both cohorts. The mediating effect of each eating behaviour on the association between the BMI-GRS and measured BMI was assessed using the Sobel test. Additionally, we tested for interactions between each eating behaviour and the BMIGRS on BMI.

Results: The association between the BMI-GRS and BMI was mediated by both emotional eating (EDEN: P-Sobel $=0.01$; Fenland: P-Sobel=0.02) and uncontrolled eating (EDEN: P-Sobel=0.04; Fenland: P-Sobel $=0.0006$ ) in both sexes combined. Cognitive restraint did not to mediate this association (P-Sobel $>0.10$ ), except amongst women in EDEN (P-Sobel=0.0009). Cognitive restraint modified the relationship between the BMI-GRS and BMI amongst men (EDEN P-interaction $=0.0001$; Fenland: P-interaction $=0.04$ ) and women in Fenland (P-interaction $=0.0004)$. By tertiles of cognitive restraint, the association between the BMI-GRS and BMI was highest in the lowest tertile of cognitive restraint, and weakest in the highest tertile of cognitive restraint.

Conclusions: Genetic susceptibility to obesity was partially mediated by the 'appetitive' eating behaviour traits (uncontrolled and emotional eating) and, in 3 of the 4 population groups studied, was modified by cognitive restraint. High levels of cognitive control over eating appear to attenuate the genetic susceptibility to obesity. Future research into interventions designed to support cognitive restraint might help to protect genetically susceptible individuals from weight gain.

\section{Disentangling genetic and environmental influences on parent personality and parenting behaviors}

Amanda Griffin, The Pennsylvania State University, United States; Amanda Ramos, The Pennsylvania State University, United States; Amanda Broderick, The Pennsylvania State University, India; Jennifer Ulbricht, The George Washington University, United States; Jody Ganiban, The George Washington University, United States; Erica Spotts, National Institutes of Health, United States; Paul Lichtenstein, Karolinska Institutet, Sweden; David Reiss, Yale University, United States; Jenae Neiderhiser, The Pennsylvania State University, United States

Understanding the determinants of parenting has been the focus of developmental and genetically informed studies for the past several decades. Examining how personality of the parent may contribute to their parenting behavior can help to clarify if parenting is simply another "personality" construct as opposed to a distinct behavior that is shaped by many different factors. The present study used a sample of adult twin pairs, each with an adolescent child (matched to co-twin same-sex child age within 4 years), collected as part of the Twin/ Offspring Study in Sweden (TOSS); N=909 twin pairs; (N=386 MZ pairs, $63 \%$ female, $\mathrm{M}$ age $=44.89$ years, $\mathrm{SD}=4.89)$ to examine genetic and environmental influences on the association between personality and parenting. Twins reported on the Temperament and Character Inventory harm avoidance subscale and the Karolinska Scales of Personality irritability, verbal, and indirect aggression subscales. Composites of parent reports of parental warmth and negativity, derived from factor scores of six scales of parent-adolescent relationship were examined. Aggressive personality and harm avoidance were correlated with negative and warm parenting $(r=.31$ and .24 , p 0.001, respectively) and were thus subjected to biometric analysis. Aggressive personality and parental negativity were explained by genetic and nonshared environmental influences $(\mathrm{A}=46 \% \mathrm{E}=54 \%$; $\mathrm{A}$ $=36 \% \mathrm{E}=64 \%$, respectively) with a significant genetic $\left(r_{A}=.44\right)$ and nonshared environmental $\left(\mathrm{r}_{\mathrm{E}}=.22\right)$ correlation. Harm avoidance and parental warmth were also explained by genetic and nonshared environmental influences $(\mathrm{A}=44 \%, \mathrm{E}=54 \% ; \mathrm{A}=43 \%, \mathrm{E}=54 \%$, respectively) with significant correlations between the genetic $\left(\mathrm{r}_{\mathrm{A}}\right.$ $=.28)$ and nonshared environmental $\left(\mathrm{r}_{\mathrm{E}}=.20\right)$ factors. Findings indicate that personality types have a differential shared genetic and environmental influence with parenting behaviors.

\section{Exploring neighborhood environmental influences on reading comprehension}

Callie Little, Florida State University, United States; Sara Hart, Florida State University, United States; Jeanette Taylor, Florida State University, United States

Purpose: Bioecological theory suggests that development, including reading development, occurs through interactions between individuals and proximal environmental contexts, though neighborhood characteristics are often underrepresented within studies of proximal processes on reading outcomes. The present study sought to further knowledge of the environmental influences on reading comprehension by proposing several hypothesized risk and protective aspects of the neighborhood environment and using a novel combination of techniques to explore their association with reading comprehension.

Methods: Participants were obtained from the longitudinal Florida Twin Project on Reading and included 2215 twin pairs (751 MZ, 1,464 DZ). Reading comprehension data from the Florida Comprehensive Assessment Test (FCAT) standardized assessment and family-level SES were obtained for the 2013-2014 school year. Community-level SES was obtained from 2015 U.S. census data. Geocoding techniques were used to determine Euclidean distance between twin's homes and several hypothesized positive and negative neighborhood features (i.e. parks, libraries, factories, shelters). Next, using a model which estimates the proportion of variance in reading predicted by a measured aspect of neighborhood quality, while simultaneously estimating A, C, and E, we examined whether distance to neighborhood characteristics accounted for a portion of the shared environmental influences on reading comprehension.

Results and Discussion: Results indicated that of the proposed neighborhood features, distance to shelters was the sole predictor of reading comprehension after accounting for family- and communitylevel SES. Additionally, distance to shelters explained a small, yet significant proportion of shared environmental influences (.01), suggesting shelters may create environmental conditions such as disorder or chaos in the neighborhood which could have important consequences for children's reading. Chaos in the nearby, external environment may influence children's reading through noise, disruption and distraction, potentially influencing children's ability to concentrate while reading or limiting the time children engage in reading at home.

\section{GWAS results for educational attainment aid in identifying genetic heterogeneity of schizophrenia}

Vikas Bansal, Max Planck Institute of Experimental Medicine, Germany; Marina Mitjans, Max Planck Institute of Experimental Medicine, Germany; Casper Burik, Vrije Universiteit Amsterdam, Netherlands; Martin Begemann, Max Planck Institute of Experimental Medicine, Germany; Stefan Bonn, German Center for Neurodegenerative Diseases (DZNE), Germany; Richard Karlsson Linnér, Vrije Universiteit Amsterdam, Netherlands; Aysu Okbay, Vrije Universiteit Amsterdam, Netherlands; Niels Rietveld, Erasmus 
University Rotterdam, Netherlands; Stephan Ripke, Charité Universitätsmedizin Berlin, Germany; Ronald de Vlaming, VU, Netherlands; Michel Nivard, Vrije Universiteit Amsterdam, Netherlands; Hannelore Ehrenreich, Max Planck Institute of Experimental Medicine, Germany; Philipp Koellinger, Vrije Universiteit Amsterdam, Netherlands

Higher educational attainment (EA) is known to have a protective effect on the severity of schizophrenia (SZ). However, recent studies have found a small positive genetic correlation between EA and SZ. We investigated possible causes of this counterintuitive finding using genome-wide association (GWAS) results for EA and SZ (N= 443,581) and a replication cohort (1,169 controls and 1,067 cases) with high-quality SZ phenotypes. We found genetic dependence between EA and SZ that cannot be explained by chance, linkage disequilibrium, or assortative mating. Instead, several genes seem to have pleiotropic effects on EA and SZ, but without a clear pattern of sign concordance. Genetic heterogeneity in both phenotypes is the most likely explanation of this finding. This insight can be exploited by using a combination of EA and SZ GWAS results to improve the polygenic prediction of clinical symptoms and disease severity of SZ. In particular, although a polygenic score for $\mathrm{SZ}$ is robustly associated with case-control status, it does not predict any of the SZ symptoms or disease severity. In contrast, co-dependent polygenic scores that split the SZ score into two parts based on the sign concordance of SNPs for SZ and EA predict symptoms and disease severity in patients to some extent. Furthermore, using EA as a proxy-phenotype for SZ, we isolate FOXO6 and SLITRK1 as additional statistically plausible candidate genes for SZ.

\section{The etiological structure of cognitive- neurophysiological impairments in ADHD in adolescence and young adulthood}

Giorgia Michelini, King's College London, Institute of Psychiatry, Psychology \& Neuroscience, United Kingdom; Celeste Cheung, King's College London, MRC Social, Genetic and Developmental Psychiatry Centre, Institute of Psychiatry, Psychology and Neuroscience, London, UK, United Kingdom; Viryanaga Kitsune, King's College London, MRC Social, Genetic and Developmental Psychiatry Centre, Institute of Psychiatry, Psychology and Neuroscience, London, UK, United Kingdom; Daniel Brandeis, Department of Child and Adolescent Psychiatry and Psychotherapy, Central Institute of Mental Health, Medical Faculty Mannheim/Heidelberg University, Mannheim, Germany, Germany; Tobia Banaschewski, Department of Child and Adolescent Psychiatry and Psychotherapy, Central Institute of Mental Health, Medical Faculty Mannheim/Heidelberg University, Mannheim, Germany, Germany; Grainne McLoughlin, King's College London, MRC Social, Genetic and Developmental Psychiatry Centre, Institute of Psychiatry, Psychology and Neuroscience, London, UK, United Kingdom; Philip Asherson, King's College London, MRC Social, Genetic and Developmental Psychiatry Centre, Institute of Psychiatry, Psychology and Neuroscience, London, UK, United Kingdom; Fruhling Rijsdijk, King's College London, MRC Social, Genetic and Developmental Psychiatry Centre, Institute of Psychiatry, Psychology and Neuroscience, London, UK, United Kingdom; Jonna Kuntsi, King's College London, MRC Social, Genetic

and Developmental Psychiatry Centre, Institute of Psychiatry, Psychology and Neuroscience, London, UK, United Kingdom

Attention-deficit/hyperactivity disorder (ADHD) is associated with multiple cognitive impairments. Previous studies have identified two partially separable familial factors underlying the cognitive impairments in childhood combined-type ADHD. The extent to which these cognitive impairments share familial influences with ADHD beyond childhood is unknown. Here, we investigate the etiological structure of cognitive-neurophysiological impairments in persistent ADHD in adolescence and young adulthood. In a sample of 356 participants from ADHD and control sibling pairs (11-27 years), factor analyses and multivariate familial models were run on data on IQ, digit span forward (DSF), digit span backward (DSB), and cognitive-performance and event-related potential (ERP) measures from a cued continuous performance task, an arrow flanker task and a fourchoice reaction time task. Three familial factors (cF1-3) were identified, which captured the familial covariation of ADHD with nine cognitive-ERP measures. $\mathrm{cF} 1$ loaded on IQ, mean reaction time (MRT) and reaction-time variability (RTV); $\mathrm{cF} 2$ on DSF and DSB; and $\mathrm{cF} 3$ on number of errors and ERPs of inhibitory control (NoGoP3) and error processing (error-related negativity; ERN). All three factors showed significant familial overlap with ADHD $\left(\mathrm{r}_{\mathrm{CF} 1-\mathrm{ADHD}}\right.$ $=.50 ; \mathrm{rCF} 2-\mathrm{ADHD}=-.36 ; \mathrm{rCF} 3-\mathrm{ADHD}=-.66)$. Non-familial influences showed the same factor structure, except for IQ that clustered with digit span measures. Non-familial influences on MRT and RTV largely overlapped with those on ADHD, while other non-familial effects were largely measure-specific. By using a broad range of cognitive and neurophysiological measures within a family study, we identified three partially separable familial factors that substantially captured influences shared between cognitive-neurophysiological measures and ADHD in adolescence and adulthood. These results reveal multiple familial and non-familial processes underlying cognitive and brain impairments in persistent ADHD.

\section{On the etiology of self-esteem: genetic and environmental effects}

Rainer Riemann, Bielefeld University, Germany; Anke Hufer, Bielefeld University, Germany; Christian Kandler, Medical School Berlin, Germany; Amelie Nikstat, Bielefeld University, Germany; Chris Hopwood, University of California, Davis, United States; Wiebke Bleidorn, University of California, Davis, United States

Self-esteem is related to a variety of positive life outcomes, including social integration, job success, or health. In contrast to the broad literature on the consequences of self-esteem, little is known about the genetic and environmental factors that shape the origin and development of self-esteem. Using data from the TwinLife project $(\sim$ 4,000 twin families) and an extended twin family design (ETFD), we examine the interplay of social (environmental) and genetic influences on self-esteem. In addition, using moderated ETFD models, we tested whether the genetic and environmental influences on self-esteem vary as a function of age, gender, and socio-economic status (SES). Results suggest that individual differences in self-esteem are influenced by genetic, nonshared environmental and shared environmental factors. Perhaps surprising, the latter were not passed from parent to offspring but reflected the influence of non-parental factors such as shared demographic factors. We further documented moderation effects of age, gender, and SES on the genetic and environmental influences on self-esteem. We discuss how genetic and environmental contributions to self-esteem might be more richly conceptualized as dynamic systems of gene-environment interplay. In addition to cohort effects, comparing 17 years old with 23/24 year old twins, we study interactions of genes with family-of-origin socioeconomic status (SES, income or education) using a nuclear twin family design that includes not only data from the twins but also data from their parents and a sibling. As expected we find substantial genetic and environmental effects and significant age trends indicating larger genetic effects on the older twins, but only marginal effects 
of gene $\times$ environment interactions. Non-shared environmental effects are examined by studying monozygotic twins discordant for environmental variables.

\section{Examination of alternative models of $\mathbf{G} \times \mathbf{E}$ moderation}

Chelsea Sawyers, Virginia Commonwealth University, United States; Michael Neale, Virginia Commonwealth University, United States

The Cholesky parameterization has been used to examine the potential shared latent structure of two variables. Several modifications have been implemented to examine gene $\times$ environment interactions with the aim of reducing overall error rates, especially in the case of moderators correlated between twins. We propose an alternative model for consideration when examining the moderating effect of one definition variable on the relationship between two other variables. A model allowing for correlations between the latent genetic and environmental factors of two variables would be appropriate to use when there is no a priori justification for testing one direction of influence over another. Additionally, moderation between the two variables by a third can be examined on each of the variables, as well as on the correlations between their latent variables. This 'correlated factors' (CF; bivariate) moderation model does not lend itself easily to comparison with a classic Cholesky (trivariate) decomposition to examine the relationships between three variables. Rather, the correlated factors model was compared to the "extended bivariate' (EB) model proposed by van der Sluis et al. (2012); with modification accounting for two variables rather than the original univariate designs. Since it is often the case that moderators of interest are correlated between twins, the alternative model is applied to all models tested during the simulations. Using simulated data (500 simulations; 1000 mono/dizygotic pairs), model fits were compared between $\mathrm{CF}$ and EB models. Ability to accurately detect simulated moderation on covariances, means and correlations was tested across all models. Likelihood ratio tests were performed to compare goodness of fit. Overall, the CF model provides a more readily interpreted framework for the study of multivariate GxE interactions.

\section{References}

van der Sluis et al., 2012. A Note on False Positives and Power in G $\times$ E Modelling of Twin Data. Behavior Genetics. 42:170-186.

\section{Social support and mental health in adolescence are correlated for genetic, as well as environmental, reasons}

Adele Wang, University of Bristol, United Kingdom; Oliver Davis, University of Bristol, United Kingdom; Claire Haworth, University of Bristol, United Kingdom

In late adolescence many individuals experience major life changes, such as moving away from home, transitioning from education to employment and starting a family. Social support during this time is important in ensuring that individuals adjust to the environmental stressors that these life events can bring. However, as adolescents transition into adulthood, their social network structure changes. Therefore, late adolescence is a crucial developmental stage to investigate the aetiology of social support and the link between support and psychological adjustment. We collected data from 1,215 18-year-old twin pairs from the Twins Early Development Study to examine this. Individuals completed measures of wellbeing and depression as well as two indices of social support: perceived support and frequency of social contact. Both support indices were moderately heritable (55 and 49\%, respectively). Phenotypic and genetic correlations between support and mental health constructs were moderate, with shared genetic influences explaining between 57 and $85 \%$ of the phenotypic correlation (mean $\mathrm{r}=.46$ ). Genetic correlations were higher between perceived support and mental health (mean $r_{A}=0.75$ ), than between frequency of social contact and mental health (mean $r_{A}=0.54$ ), reflecting the phenotypic pattern seen here and in previous literature. Finding genetic influence on social support suggests the presence of gene-environment correlation whereby individuals create, and perceive their supportive environment based upon their genetic predispositions.

\section{Dyadic perspectives in genetically informed research}

Jessica Salvatore, Virginia Commonwealth University, United States

Fundamental aspects of the human experience and the development of the self occur in the context of romantic relationships. Central aspects of these relationships (e.g., trust, commitment, satisfaction, stability) are dyadically constructed, cautioning against reductionist approaches that focus on the characteristics of either partner in isolation. There is growing interest in understanding relationship outcomes in behavioral genetic research; however, there has not yet been an in-depth treatment of whether and how traditional behavioral genetic models are appropriate for understanding romantic relationship outcomes. The overarching goals of this paper are to: (1) describe the current state of genetically informative research for dyadic romantic relationship outcomes (e.g., marital status, relationship satisfaction) with a particular emphasis on the limitations of the individual-focused statistical models that have generated this knowledge; and (2) to outline an agenda for future research in this area that incorporates a dyadic perspective. This work addresses an important gap in the literature, which is the disconnect between decades of behavioral research showing that relationship outcomes are more than the sum of the individual partners' characteristics, and the typical approach of treating the individual as the unit of analysis in genetically informed designs.

\section{Genetic influence on intergenerational educational attainment}

Ziada Ayorech, King's College London, Institute of Psychiatry, Psychology \& Neuroscience, United Kingdom; Eva Krapohl, King's College London, Institute of Psychiatry, Psychology \& Neuroscience, United Kingdom; Robert Plomin, King's College London, Institute of Psychiatry, Psychology \& Neuroscience, United Kingdom; Sophie Von Stumm, Goldsmith's University of London, United Kingdom

Using twin (6,105 twin pairs) and genomic (5,825 unrelated individuals) analyses, we tested for genetic influences on the parentoffspring correspondence in educational attainment. Genetics accounted for nearly half of the variance in intergenerational educational attainment. A genome-wide polygenic score (GPS) for years of education from a recent genome-wide association study (Okbay et al., 2016) was also associated with intergenerational educational attainment: The highest and lowest GPS means were found for offspring in stably educated $(\mathrm{M}=0.43 ; \mathrm{SD}=0.97)$ and stably uneducated $(\mathrm{M}=-$ $0.19 ; \mathrm{SD}=0.97$ ) families, while the GPS scores fell in between for families that were upwardly mobile (parents not university educated, offspring taking A-levels) $(\mathrm{M}=0.05 ; \mathrm{SD}=0.96)$ and downwardly mobile (parents university educated, offspring not taking A-levels) $(\mathrm{M}=0.28 ; \quad \mathrm{SD}=1.03)$. Genetic influences on intergenerational 
educational attainment can be viewed as an index of equality of educational opportunity.

\section{Monozygotic twins discordant for educational attainment: definitions and other challenges}

Brian Byrne, University of New England, Australia; William Coventry, University of New England, Australia; Connie Suk-Han Ho, The University of Hong Kong, Hong Kong; Sally Larsen, University of New England, Australia; Richard Olson, University of Colorado Boulder, United States

Discordant monozygotic twins offer a unique opportunity to examine environmental influences on human traits. We report on our attempts to exploit that opportunity within a database of Australian twins assessed longitudinally across four school grades in literacy and numeracy in nationally-administered "high-stakes" testing. The first challenge is to define discordancy within pairs; issues include degree of separation, longitudinal consistency, and consistency across assessment domains. Adopting a working definition of 1SD separation on one occasion, we show that longitudinal consistency is relatively weak, raising the issue of whether single instances of discordancy should be considered of interest or assigned to "error." Insisting on consistency across domains runs the risk of missing environmental factors affecting just one domain, numeracy, for instance. We entertain the hypothesis that cross-domain consistency might signal broader biological factors such as illness in one twin but also show that testing that hypothesis is challenged by a possible causal path from literacy to numeracy. The second challenge is to identify the null hypothesis, and we suggest that it is that there are as many environmental factors in operation as there are pairs of discordant twins. A third challenge, perhaps particular to our database, is the validity of the high-stakes tests against school-based assessments. A fourth challenge is whether there are factors at work that throw doubt on the generalisability of our observations to students in general, such as twin competitiveness. Finally, the relatively modest number of consistently discordant pairs for highly heritable traits like literacy and numeracy (despite a database of several 1000 families) challenges standard quantitative analyses and requires more reliance on interviews, with their subjective elements. We report the results of a series of parent interviews that shed light on these issues.

\section{Drinking-discordant MZ twin pairs: a forty-year follow-up}

Richard Rose, Indiana University, United States; Richard Viken, Psychological \& Brain Sciences, Indiana University, United States; Jaakko Kaprio, University of Helsinki, Finland

Alcohol exposure is adolescence/early adulthood predicts adverse outcomes later in life. But the persistence and causal nature of these associations remain uncertain. We address those uncertainties with a 40-year follow-up of drinking-discordant monozygotic twin pairs assessed across multiple occasions from 1975 to 2016. From the population-based Older Finnish Twin Cohort, we identified 171 pairs, born 1950-1957, known or presumed to be MZ and meeting criteria for drinking-discordance in 1975 when ages 18-25. At baseline, the mean monthly consumption reported by the heavierdrinking twins in these discordant pairs was 556 grams/month versus $170 \mathrm{~g} /$ month for co-twins. Twins in all pairs completed a postal questionnaire in 2011-2012, and in 113 pairs, both twins completed a semi-structured telephone interview during 2014-2016. Results confirm significant long-term continuity of both individual and intrapair differences in alcohol exposure with associated intra-pair differences in measured outcomes 40 years later. Individual differences in consumption correlated about 0.44 in both twin brothers and sisters across a 37-year span, and within-pairs, the heavier drinking twin in 1975 likely remained the heavier drinking twin on follow-up (p .018). Scores on a Nordic questionnaire screen for alcohol problems were significantly higher among co-twins who reported heavier drinking decades earlier. Heavier-drinking co-twins reported significantly more alcohol disorder symptoms on interview $(\mathrm{t}=2.78$, p .009), significantly lower scores on a Satisfaction with Life Scale $(t=1.86, p .034)$, and significantly higher scores indicative of poorer mental health on the 12-item General Health Questionnaire ( $t=1.96$, $p$.025). The correlation of signed intra-pair differences in consumption reported in 1975 with the number of alcohol disorder symptoms tallied from interviews in 2014-2016 was highly significant $(\mathrm{r}=.26, \mathrm{p}$.003). Controlling for familial/genetic confounds, these results are consistent with inferences that heavy alcohol exposure in late adolescence and early adulthood causes adverse consequences across life course development. Those results encourage studies of epigenetic changes and paired comparisons of brain scans in this informative sample of drinking-discordant Finnish MZ twins.

\section{Differences in exam performance between school types mirrors genotype differences between them}

Emily Smith-Woolley, King's College London, United Kingdom; Eva Krapohl, King's College London, Institute of Psychiatry, Psychology \& Neuroscience, United Kingdom; Saskia Selzam, King's College London, United Kingdom; Kaili Rimfeld, King's College London, Institute of Psychiatry, Psychology \& Neuroscience, United Kingdom; Jean-baptiste, Pingault University College London, United Kingdom; Sophie Von Stumm, Goldsmith's University of London, United Kingdom; Kathryn Asbury, York University, United Kingdom; Philip Dale, University of New Mexico, United States; Yulia Kovas, Goldsmiths, University of London, United Kingdom; Robert Plomin, King's College London, Institute of Psychiatry, Psychology \& Neuroscience, United Kingdom

On average, independent and grammar schools out-perform their state-funded, non-selective counterparts in national exams, including the General Certificate of Secondary Education (GCSEs), taken in the UK at age 16. These differences are often attributed to selection factors such as student ability or socio-economic status, however the possible role of DNA differences has not yet been considered. Here, for the first time, we use a UK-representative sample of 5,825 unrelated, genotyped individuals to investigate GCSE and genotype differences between three types of schools in the UK: state-funded schools that are non-selective, state schools that are selective ('grammar'), and private schools that are selective ('independent'). We created a polygenic score derived from a 2016 genome-wide association study of years of education and found that GCSE differences between schools were mirrored in the polygenic score differences between schools, with non-selective state schools students showing significantly lower polygenic scores compared with those in grammar schools $(\mathrm{d}=0.37)$ and independent schools $(d=0.35)$. Furthermore, we find that after controlling for student selection factors (general intelligence, prior ability in English and Maths, and SES), school type explains less than $1 \%$ of variance in GCSE scores. These results suggest that GCSE differences between school types primarily reflect genetically driven selection factors between schools, rather than educational value added by the school types. 


\section{Performing Mendelian randomization using structural equation models}

David Evans, University of Queensland, Australia; Nicole Warrington, University of Queensland, Australia

Mendelian randomization (MR) is a method of estimating the causal effect of modifiable environmental exposures on medically relevant outcomes, identifying molecular biomarkers that are likely to be causal for disease, and determining the suitability of drug targets for pharmacological intervention. However, MR studies are currently performed using very simple statistical methods based on e.g. two stage least squares and Wald ratios. These approaches lack flexibility to model more complicated causal networks involving many different variables, bidirectional relationships, and horizontal pleiotropy, which in some cases may invalidate analyses and bias estimates of causal effects. Structural Equation Modelling (SEM) is a very flexible statistical tool that allows the modelling of complex linear dependencies between variables and the estimation of causal effects. Despite the potential advantages, SEM has yet to have been employed in MR studies except in the simplest of situations. In this presentation I show how SEM can be combined with Mendelian randomization principles to estimate complicated causal effects that may not be easy to estimate using simple ratio or two stage least squares models. I illustrate the advantages afforded by SEM using large scale data from the UK Biobank Study.

\section{Large-scale genetic study of risk tolerance and risky behaviors identifies new loci and reveals shared genetic influences}

Richard Karlsson Linnér, Vrije Universiteit Amsterdam, Netherlands; Pietro Biroli, University of Zurich, Switzerland; Mark Fontana, University of Southern California, United States; Anke Hammerschlaag, Vrije Universiteit Amsterdam, Netherlands; Edward Kong, Harvard University, United States; Maël Lebreton, Universiteit van Amsterdam, Netherlands; Fleur Meddens, Vrije Universiteit Amsterdam, Netherlands; Michel Nivard, Vrije Universiteit Amsterdam, Netherlands; Aysu Okbay, Vrije Universiteit Amsterdam, Netherlands; Niels Rietveld, Erasmus University Rotterdam, Netherlands; Erdogan Taskesen, Vrije Universiteit Amsterdam, Netherlands; Patrick Turley, Broad Institute of MIT and Harvard, United States; Ronald De Vlaming, Vrije Universiteit Amsterdam, Netherlands; Robbee Wedow, University of Colorado Boulder, United States; Abdel Abdellaoui, Vrije Universiteit Amsterdam, Netherlands; Yanchun Bao, University of Essex, United Kingdom; Laura Buzdugan, University of Zurich, Switzerland; Chiayen Chen, Massachussetts General Hospital, United States; Peter Eibich, University of Oxford, United Kingdom; Pierre Fontanillas, 23andMe, United States; Peter Joshi, University of Edinburgh, United Kingdom; Ville Karhunen, University of Oulu, Finland; Christina Lill, University of Lübeck, Germany; Gerardus Meddens, Team Loyalty BV, Netherlands; Gerard Muntané, Universitat Pompeu Fabra, Spain; Sandra Sanchez-roige, University of California, San Diego, United States; Frank Van Rooij, Erasmus University Rotterdam, Netherlands; Murray Stein, University of California, San Diego, United States; Adam Auton, 23andMe, United States; David Clark, University of Edinburgh, United Kingdom; Andrew Conlin, University of Oulu, Finland; Conor Dolan, Vrije Universiteit Amsterdam, Netherlands; Urs Fischbacher, University of Konstanz, Germany; Patrick Groenen, Erasmus University Rotterdam,
Netherlands; Gregor Hasler, University of Bern, Switzerland; Albert Hofman, Erasmus University Rotterdam, Netherlands; M. Arfan Ikram, Erasmus Medical Center, Netherlands; Sonia Jain, University of California, San Diego, United States; Robert Karlsson, Karolinska Institutet, Sweden; James Mackillop, McMaster University, Canada; Minna Männikkö, University of Oulu, Finland; Carlos Morcillosuarez, Universitat Pompeu Fabra, Spain; Klaus Schmidt, University of Munich, Germany; Melissa Smart, University of Essex, United Kingdom; Matthias Sutter, University of Cologne, Germany; Roy Thurik, Erasmus University Rotterdam, Netherlands; André Uitterlinden, Erasmus Medical Center, Netherlands; Jon White, University College London, United Kingdom; Harriet de Wit, University of Chicago, United States; Lars Bertram, University of Lübeck, Germany; Dorret Boomsma, Vrije Universiteit Amsterdam, Netherlands; Ernst Fehr, University of Zurich, Switzerland; Magnus Johannesson, Stockholm School of Economics, Sweden; Meena Kumari, University of Essex, United Kingdom; Patrik KEMagnusson, Karolinska Institutet, Sweden; Arcadi Navarro, Universitat Pompeu Fabra, Spain; Abraham Palmer, University of California, San Diego, United States; Daniel Schunk, Johannes Gutenberg University, Germany; Rauli Svento, University of Oulu, Finland; Henning Tiemeier, Erasmus Medical Center, Netherlands; Paul Timmers, University of Edinburgh, United Kingdom; Robert Ursano, Uniformed Services University Health Science, United States; Gert Wagner, Max Planck Institute for Human Development, Germany; James Wilson, University of Edinburgh, United Kingdom; James Lee, University of Minnesota Twin Cities, United States; Jake Gratten, The University of Queensland, Australia; David Laibson, Harvard University, United States; Danielle Posthuma, Vrije Universiteit Amsterdam, Netherlands; David Cesarini, New York University, United States; Daniel Benjamin, University of Southern California, United States; Philipp Koellinger, Vrije Universiteit, Netherlands; Jonathan Beauchamp, University of Toronto Canada

Risk tolerance is an important variable in the behavioral sciences, but few genetic variants have so far been found to robustly associate with it or with risky behaviors. We, the Social Science Genetic Association Consortium (SSGAC), conducted genome-wide association studies (GWAS) of general risk tolerance $(n=108,689)$ and four risky behaviors: automobile speeding propensity, alcoholic drinks per week, whether one has ever been a smoker, and the lifetime number of sexual partners $(n=93,625-186,102)$. Our GWASs identified several genome-wide significant loci associated with these outcomes or their first principal component. We report evidence of substantial pleiotropy between general risk tolerance and the four risky behaviors. Our results begin to elucidate the biological mechanisms that underlie variation in risk tolerance and risky behaviors.

\section{Sociology and genetics}

\section{Torkild Lyngstad, University of Oslo/NORMENT, Norway}

In recent years, the interest for genetically informed studies has increased markedly in sociology and allied disciplines. Twin and family of various types are more frequently used, and in the last few years also molecular genetic data. A number of important research areas in sociology, such as intergenerational transmission, status attainment and social demography, all invite genetically informed studies and use of genetic data. The purpose of this symposium is to showcase some current work and provide directions for the future. The symposium includes presentations of current work social scientists are doing with twin/family data or genomic data. 
Are your covariates under control? How normalization can reintroduce covariate effects

Oliver Pain, Birkbeck College/London School of Hygiene and Tropical Medicine, United Kingdom; Frank Dudbridge, Leicester University, United Kingdom; Angelica Ronald, Birkbeck College, University of London, United Kingdom

Many statistical tests rely on the assumption that the residuals of a model are normally distributed. One of the most popular approaches to satisfy the normality assumption is rank-based inverse normal transformation (INT) of the dependent variable. It is often desirable to adjust for covariates in analysis, such as principal components of ancestry in genetic studies. When a transformation to normality is used, the covariates may be included in the analysis model after transformation, or alternatively they may be regressed against the response as a preliminary step and the residuals then transformed to normality. This study investigates the effect of applying rank-based INT to the dependent variable either before or after controlling for covariate effects. This was achieved by assessing the correlation between the dependent variable and covariates when the covariate effects are regressed from the dependent variable either before or after the dependent variable is transformed. Three factors predicted to affect the outcome of this process were investigated: the proportion of tied observations in the dependent variable, the original skew of the dependent variable, and the original correlation between the dependent variable and covariate. This procedure was performed using both simulated variables and real data examples. The results demonstrated that applying rank-based INT to the dependent variable residuals in almost all situations re-introduces a linear correlation between the dependent variable and covariates that will lead to increased type- 1 errors and reduced power. An alternative approach is recommended that allows a normally distributed dependent variable to be linearly uncorrelated with covariates.

\section{Design and implementation of a customized genotyping array for imputation-based genome-wide association studies: the avera-NTR global screening array}

Jeffrey Beck, Avera Institute for Human Genetics, United States; Erik Ehli, Avera Institute for Human Genetics, United States; Hamdi Mbarek, Vrije Universiteit Amsterdam, Netherlands; Noah Kallsen, Avera Institute for Human Genetics, United States; Shanna Peyton, Avera Institute for Human Genetics, United States; Abdel Abdellaoui, Vrije Universiteit Amsterdam, Netherlands; Gonneke Willemsen, Vrije Universiteit Amsterdam, Netherlands; Iryna Fedko, Vrije Universiteit Amsterdam, Netherlands; Eco De Geus, Vrije Universiteit Amsterdam, Netherlands; Dorret Boomsma, Vrije Universiteit Amsterdam, Netherlands; Gareth Davies, Avera Institute for Human Genetics, United States; Jouke Hottenga, Vrije Universiteit Amsterdam, Netherlands

Prior to the inception of genome wide association studies (GWAS), the primary method for identifying causal genetic variants was through inheritance studies of genetic linkage. Single gene disorders have been well characterized through linkage analyses; however, despite optimism about linkage designs for quantitative traits, the approach was not successful in studying human complex traits and diseases. With the increases in sample size, GWAS have gained utility as an informative and cost effective approach for identifying genetic variants associated with complex phenotypes. The fundamental method for generating data for GWAS is through high-density single nucleotide polymorphism (SNP) genotyping followed by imputation. The Avera Institute for Human Genetics has joined the
Infinium Global Screening Array (GSA) Consortium initiated by Illumina, Inc., to gain access to a customized array containing highly predictive markers for translational research applications. In conjunction with collaborators at the Netherlands Twin Register (NTR), the Avera-NTR GSA array was designed to include a core imputation backbone and thousands of other markers of interest concerning Alzheimer's disease, drug metabolism, fertility and twinning, and schizophrenia. Initial validation of the array was assessed by checking concordance, coverage, and imputation quality using the Genome of the Netherlands reference sequence. Preliminary application of the array was carried out with DNA extracted from a variety of tissues, including blood, buccal, and saliva samples. As of current, 10,484 samples (7,009 buccal, 3,056 blood, 419 saliva) have been genotyped on the Avera-NTR GSA array, with an overall weighted average call rate of $99.19 \%(99.04,99.60,98.66 \%$, respectively) for 697,564 markers. In the future, investigative GWAS, for a multitude of human complex traits, including genetic variants associated with dizygotic twinning, will be conducted with the genotypic data generated by the Avera-NTR GSA array which has shown great utility in generating high-quality SNP data from multiple tissue types.

\section{Aggression and co-occurring behaviors in the school setting: a multi-site population-based investigation}

Alyce Whipp, University of Helsinki, Finland; Eero Vuoksimaa, University of Helsinki, Finland; Tellervo Korhonen, University of Helsinki, Finland; Richard Rose, Indiana University United States; Lea Pulkkinen, University of Jyvaskyla, Finland; Eva Krapohl, King's College London, Institute of Psychiatry, Psychology \& Neuroscience, United Kingdom; Eveline De Zeeuw, Vrije Universiteit Amsterdam, Netherlands; Toos Van Beijsterveldt, Vrije Universiteit Amsterdam, Netherlands; Koen Bolhuis, Erasmus Medical Center, Netherlands; Henning Tiemeier, Erasmus Medical Center, Netherlands; Robert Plomin, King's College London, Institute of Psychiatry, Psychology \& Neuroscience, United Kingdom; Jaakko Kaprio, University of Helsinki, Finland; Meike Bartels, Vrije Universiteit Amsterdam, Netherlands; Dorret Boomsma, Vrije Universiteit Amsterdam, Netherlands

No abstract available

\section{Overlap between anorexia and bulimia nervosa: understanding genetic and environmental effects using a population registry in Sweden}

Shuyang Yao, Karo, Sweden; Ralf Kuja-Halkola, Karolinska Institutet, Sweden; Claes Norring, Karolinska Institutet, Sweden; Andreas Birgegård, Karolinska Institutet, Sweden; Cynthia Bulik, Karolinska Institutet, Sweden; Henrik Larsson, Karolinska Institutet, Sweden

Background: Symptomatic flux and familial co-aggregation of anorexia nervosa and bulimia nervosa suggests etiological overlap between these eating disorders. One previous twin study based on self-ratings explored the genetic and environmental aspects of the overlap on the symptom level.sup1/sup No previous study has explored the relative contribution of genetic and environmental influences on clinically diagnosed anorexia and bulimia nervosa. Method: We applied bi-variate structural equation modeling on fullsisters $(334,433$ pairs $)$ and maternal half-sisters (57,036 pairs) that were randomly selected from the Swedish female population born 
1970-2005. Anorexia nervosa included registered clinically diagnosed typical and atypical anorexia nervosa, and bulimia nervosa included registered clinically diagnosed typical and atypical bulimia nervosa.

Results: In the study sample, heritability was estimated as .44 for anorexia nervosa $(95 \%$ confidence interval $[\mathrm{CI}]=.38-.51)$ and .34 for bulimia nervosa (95\% CI .25-.44). Nonshared environmental effects explained the remaining phenotypic variance (anorexia nervosa .56 , $95 \% \mathrm{CI}=.49-.62$; bulimia nervosa $.66,95 \% \mathrm{CI}=.56-.75)$. The phenotypic correlation between the two disorders was estimated as .59 . The cross-sister-cross-disorder correlation was significantly higher in full-sisters $(.16$, s.d. $=.0019)$ than in maternal half-sisters $(.022$, s.d. $=.052$ ). Around half of the phenotypic covariance between anorexia and bulimia nervosa was attributable to genetic influences $(.52,95 \%$ $\mathrm{CI}=.41-.63)$. We observed statistically significant genetic $(.79,95 \%$ $\mathrm{CI}=.63-.95)$ and environmental $(.47,95 \% \mathrm{CI}=.37-1.00)$ correlations between these disorders.

Conclusion: In line with the only previous twin study based on selfratings, we report a moderate-to-high genetic correlation and a moderate environmental correlation between anorexia and bulimia nervosa. ${ }^{1}$ This points towards a shared mechanism that could guide future molecular genetic research and further encourage clinicians to be vigilant for symptom flux in patients with these eating disorders.

\section{References}

1. Bulik, C. M. et al. Understanding the relation between anorexia nervosa and bulimia nervosa in a Swedish national twin sample. Biol Psychiatry 67, 71-77, (2010). doi:10.1016/j.biopsych.2009.08.010

\section{The polygenic risk score for depression is associated with symptoms of antisocial personality disorder and interacts with childhood SES}

Michael Lyons, Boston University, United States; Mark Logue, Boston Univ., United States; Ruth Mckenzie, Boston Univ., United States; Rosemary Toomey, Boston Univ., United States; Matthew Panizzon, University of California, San Diego, United States; Carol Franz, University of California, San Diego, United States; Ming Tsuang, Univ. of California, San Diego, United States; William Kremen, University of California, San Diego, United States

A number of studies have demonstrated a familial relationship between depression and antisocial personality disorder (ASPD). We examined the possibility that major depression (MD) and ASPD share genetic influences. Participants were 1054 twins from the Vietnam Era Twin Registry (mean age 41.5 years (u+/u2.34)). They were administered the Diagnostic Interview Schedule III-R from which number of lifetime symptoms of MD, dysthymia, and ASPD were derived. We examined the relationship of polygenic risk scores (PRS) for depression to symptoms of MD, dysthymia, and ASPD. PRS was based on weights derived from a GWAS of MD. PRSs were computed at a range of P-value thresholds. Results are reported for the most significant threshold of $\mathrm{p} .0 .2$, which included 53,972 SNPs. We controlled for non-independence of twins using mixed models; age and race/ethnicity were used as covariates. All analyses adjusted for the first 3 principal components from SNP data to account for any cryptic population substructure. The MD PRS was significantly associated ( $p$.05) with the lifetime number of symptoms of MD, dysthymia, and ASPD. We then examined the relationship of the PRS to individual ASPD symptoms. The most significant association was with the symptom of irritable and aggressive behavior ( $p .01$ ). There was also a trend ( $p .10)$ for associations with starting fights, using a weapon in fights, and failure to conform to social norms. We investigated whether childhood SES moderates the relationship between genetic risk for depression and ASPD. We found a significant interaction between the PRS and childhood SES $(\mathrm{p}=.02)$. The relationship between depression PRS and ASPD was larger among those raised in a lower SES environment. Although the magnitudes of the associations that we observed were relatively modest, very often PRSs do not explain a great deal of the variance, even in the phenotype from which they were derived.

\section{Genetic and environmental effects on political preferences and party identification in adolescence and early adulthood: an extended twin family analysis}

Anke Hufer, Bielefeld University, Germany; Anna Elena, Kornadt Bielefeld University, Germany; Christian Kandler, Medical School Berlin, Germany; Amelie Nikstat, Bielefeld University, Germany; Rainer Riemann, Bielefeld University, Germany

Political preferences and party identification have been subject of extensive research. Both aspects have been seen as mainly socially influenced attitudes for a long time. However, recent analyses have also found substantial genetic influence on social attitudes and political traits, but still little is known about the concrete pathways through which genes and environment exert influence. Since the Classical Twin Design has several limitations, we implemented a Nuclear Twin Family Design in a study of 4097 (same-sex MZ and DZ) twins reared together, their biological parents and full siblings. The twins were 17 and 23 years old, an age which marks an important transition from adolescence to young adulthood where most people start to engage in social or political activities. This design enables us to detect additive and non-additive genetic effects simultaneously and to account for assortative mating, cultural transmission and passive gene-environment correlations as well as a twin-specific (possibly age-related) environment. We asked the participants how close they felt to the currently most successful eight German parties and arranged the parties on a left-right continuum. We found substantial additive genetic effects that increase with age while shared environmental influences are small and tend to decrease. Interestingly, direct non-genetic parental effects were significant. Concerning the twin specific environment we obtained mixed results. In addition, variables that could act as potential moderators such as sex, socioeconomic status and personality were investigated. We discuss the findings with regard to their contributions to disentangle the intertwined mechanisms that influence political attitudes.

\section{The genetic and environmental foundations of morality}

Christian Kandler, Medical School Berlin, Germany; Alexandra Zapko-Willmes, MSB Medical School Berlin, Germany

It has been consistently found that individual differences in beliefs, value priorities, and attitudes are due to both environmental and genetic influences. In this study, we investigated the effects of nature and nurture on individual differences in five universally observable but differently developed moral intuitions: Harm/Care (i.e., avoiding harm to others), Fairness/Reciprocity (i.e., doing the right things to ensure fair treatment for all), Ingroup/Loyalty (i.e., having a system that protects the social institutions of family, community, and country), Authority/Respect (i.e., complying with duty, authorities, laws, and tradition), and Purity/Sanctity (i.e., preferring religious norms, decency, and maintaining purity of mind). 
Data from 546 twins (102 monozygotic and 171 dizygotic twin pairs) who already participated in the ongoing Study of Personality Architecture and Dynamics (http://www.speady.de) were analyzed. We estimated genetic and environmental sources of individual differences in moral intuitions using a latent variable approach to control for error of measurement.

The results indicate that individual differences in Harm/Care and Fairness/Reciprocity were primarily due to genetic influences (76 and $44 \%$ ) and individual-specific environmental factors (21 and 50\%), whereas variance in Ingroup/Loyalty and Authority/Respect was primarily attributable to environmental influences shared (26 and $33 \%$ ) and not shared by twins (62 and 67\%). Purity/Sanctity showed significant genetic $(22 \%)$ and shared $(33 \%)$ as well as nonshared environmental $(45 \%)$ variance components. Genetically informative common factor analyses indicated that a common factor of Harm/ Care, Fairness/Reciprocity, and Ingroup/Loyalty mediated most of the genetic variance in these variables, whereas a common factor of Ingroup/Loyalty, Authority/Respect, and Purity/Sanctity mediated most of their shared environmental variance components. These results will be discussed against the background of the structure and theory of moral foundations and related constructs, such as value priorities and political attitudes.

(The authors received support from the Deutsche Forschungsgemeinschaft KA 4088/2-1).

\section{It really does take a village: the role of neighbors in the etiology of child antisocial behavior}

S. Alexandra, Burt Michigan State University, United States; Amber Pearson, Michigan State University, United States; Kelly Klump, Michigan State University, United States; Jenae Neiderhiser, The Pennsylvania State University, United States

Although there is growing recognition that disadvantaged contexts attenuate genetic influences on youth antisocial behavior (ASB), it is not yet clear how this dampening occurs. The current study made use of a 'geographic contagion' model to isolate specific contextual elements contributing to this effect, with a focus on neighbor behavior. Our sample included 847 families residing in or near modestly-toseverely disadvantaged neighborhoods who participated in the Michigan State University Twin Registry. Neighborhood sampling techniques were used to recruit neighbors residing within 5000 meters of a given family (the mean number of neighbors assessed per family was 13.09 , with a range of $1 \mathrm{br}$ to 47 ). Analyses revealed clear evidence of GxE by neighbor behavior, such that sibling-level shared environmental influences on child ASB increased with increasing neighbor self-reports of their own ASB, while genetic influences decreased. Put differently, neighbor ASB appears to provide such a social push towards youth ASB that it develops even in the absence of genetic risk. Sensitivity analyses further indicated that neighbor joblessness (rather than neighbor criminality) accounted for these findings. Such findings suggest that future GxE studies should incorporate social contagion theory and its dynamic social networks. br.

\section{Maternal smoking during pregnancy and offspring severe mental illness: evidence of familial confounding}

Patrick Quinn, Indiana University, United States; Martin Rickert, Indiana University, United States; Caroline Weibull, Karolinska Institutet, Sweden; Anna Johansson, Karolinska Institutet, Sweden; Paul Lichtenstein, Karolinska Institutet, Sweden; Catarina Almqvist,
Karolinska Institutet, Sweden; Henrik Larsson, Karolinska Institutet, Sweden; Anastasia Iliadou, Karolinska Institutet, Sweden; Brian D'Onofrio, Indiana University, United States

Population-based studies have demonstrated associations between exposure to maternal smoking during pregnancy and offspring bipolar disorder and schizophrenia. Although these findings could be interpreted to support the hypothesis that maternal smoking during pregnancy causally increases risk of severe mental illness in offspring, such studies may have been unable to fully rule out the possibility of familial confounding. At least some of the covariation between smoking and schizophrenia is explained by familial factors, suggesting that transmission of shared liability for both outcomes (e. g., via passive gene-environment correlation) could confound a putative environmental impact of exposure to maternal smoking. Indeed, one prior sibling comparison study failed to support an association between exposure to smoking during pregnancy and offspring schizophrenia spectrum disorders after accounting for factors shared by siblings (Meier et al., 2017, American Journal of Psychiatry, 174, 187). In the present study, we used family-based comparisons and Swedish population register data on 1,680,219 offspring born 1983-2001 to examine associations between maternal self-reported smoking during pregnancy and several indices of severe mental illness drawn from clinical diagnoses. Replicating prior population-based studies, offspring with moderate (hazard ratio [HR], 1.25; 95\% CI, 1.19-1.30) and high (HR, 1.51; 95\% CI, 1.44-1.59) maternal smoking during pregnancy exposure had greater rates of severe mental illness than did unexposed offspring. In contrast, however, sibling comparisons were weaker in magnitude and did not reach statistical significance (moderate HR, 1.09; 95\% CI, 0.94-1.26; high HR, 1.14; 95\% CI, 0.96-1.35). Cousin comparisons, associations with specific diagnoses (e.g., bipolar disorder, schizophrenia spectrum disorders), and other sensitivity analyses supported the overall pattern of results. These findings provide evidence that familial confounding explains much of the population association between maternal smoking during pregnancy and severe mental illness in offspring.

\section{BayesTwin: an $\mathbf{R}$ package for Bayesian analysis of twin data}

Inga Schwabe, Tilburg University, Netherlands; Stéphanie van den Berg, University of Twente, Netherlands

Statistical analysis of data on twins where inference is focused on determining the relative contribution of nature and nurture to individual differences has been around for a long time. Although the revolution in molecular genetics has shifted the focus of the field of behaviour genetics from twin studies to GWAS and GCTA analyses, determining the heritability coefficient for a particular trait remains relevant. Where in the last quarter of the 20supth/sup century, the focus was on using structural equation modelling (SEM) and reporting Maximum Likelihood point estimates and confidence intervals, the first decade of the 21supst/sup century has seen increased use of Bayesian modelling. This has opened up new modelling possibilities such as inference on complex models that were not easily tractable using standard frequentist techniques, including models based on Item Response Theory (IRT) or modelling gene-environment interaction in the presence of gene-environment correlation. However, this new technology with its richness of possibilities has not yet been embraced by the behaviour genetics community, partly due to a lack of standard for reporting results. To make Bayesian analysis more accessible, we introduce the $\mathrm{R}$ package BayesTwin. The package includes a wide range of twin models as well as functions that plot relevant information or determine whether the analysis was 
performed well. In all analyses included in BayesTwin, an IRT model was integrated into the genetic model to facilitate analysis on item level. The integration of such a measurement model is important since an analysis based on an aggregated measure (e.g., a sum-score based analysis) can lead to an underestimation of heritability (van den Berg et al., 2007) or the finding of spurious gene-environment interactions (Schwabe et al., 2014; Molenaar et al., 2014).

\section{Using common variant analyses (GWAS) for defining an inflammatory subtype of schizophrenia to genetically inform adjuvant anti-inflammatory treatment}

\section{Stephan Ripke, Broad Institute, United States}

Genome-wide association studies (GWAS) have been enormously successful in psychiatric research. Here we will present further advances towards our understanding of schizophrenia, through the use of optimized methodology implemented in Berlin, Germany. In a first phase we are aiming to collect 2500 individuals with schizophrenia and 2500 healthy controls within 2 years with an optimized collection strategy. Repurposed drugs for the treatment of psychiatric illness have a long history; indeed, the initial first-generation antipsychoticchlorpromazine-was originally developed to support anesthesia. Based on the proinflammatory hypothesis of schizophrenia, several small randomized placebo-controlled studies on adjuvant use of nonsteroidal anti-inflammatory drugs (NSAIDs) for schizophrenia were performed, a recent meta-analysis revealed that NSAIDs may have benefits for positive symptoms. With the method of specific polygenic risk scoring we will genetically define an inflammatory schizophrenia subgroup. We will reinvite 50 schizophrenia patients with extremely high and extremely low inflammatory risk scores and perform an adjuvant double blind treatment trial with repurposed aspirin. Here we will present the methodology for this study, involving specific polygenic risk scores.

\section{References}

Ripke, S. et al. Biological insights from 108 schizophrenia-associated genetic loci. Nature 511, 421-427 (2014).

Nitta, M. et al. Adjunctive use of nonsteroidal anti-inflammatory drugs for schizophrenia: A meta-analytic investigation of randomized controlled trials. Schizophr. Bull. 39, 1230-1241 (2013).

\section{A nuclear twin family study of cognitive abilities: results from the German TwinLife study}

Juliana Gottschling, Saarland University, Germany; Elisabeth, Saarland University, Germany; Wendy Johnson, University of Edinburgh, United Kingdom; Eric Turkheimer, University of Virginia, United States; Frank M. Spinath, Saarland University, Germany

Numerous studies of the etiology of cognitive abilities have shown that genetic influences account for approximately $50 \%$ of the variance in cognitive abilities. Research has also shown that shared environmental factors appear to be more important early in life and in childhood, whereas nonshared environmental influences as well as genetic influences become more important in early adulthood and later in life. However, most studies on the heritability of cognitive abilities relied on the classical twin model which assumes random mating (i.e., uncorrelated phenotypes of the parents), as well as the absence of gene-environment interaction and correlation, and therefore only display broad estimates of heritability. When these assumptions are not met, estimates of the heritability may be biased and potentially misinterpreted. To contribute to a deeper understanding of the sources of inter-individual differences in cognitive abilities, we leveraged a nuclear-twin family design to assess the presence of assortative mating and the presence of cultural transmission between the parent and offspring generation independent of the heritability of cognitive abilities in a sample of 4,010 twin families. Analyses were based on data from 1,837 MZ twins, 2,173 DZ twin pairs comprising four different age groups $(5,11,17$, and 23 years of age), 3,802 biological mothers and 2,456 biological fathers. Results are discussed with respect to developmental trajectories in the various age groups and the role of the family environment.

\section{Common genetic risk variants for ADHD contribute to neurodevelopmental and externalizing population traits}

Isabell Brikell, Karolinska Institutet, Sweden; Henrik Larsson, Karolinska Institutet, Sweden; Lu Yi, Karolinska Institutet, Sweden; Erik Pettersson, Karolinska Institutet, Sweden; Qi Chen, Karolinska Institutet, Sweden; Ralf Kuja-Halkola, Karolinska Institutet, Sweden; Robert Karlsson, Karolinska Institutet, Sweden; Paul Lichtenstein, Karolinska Institutet, Sweden; Joanna Martin, Karolinska Institutet, Sweden

ADHD is a highly heritable disorder, marked by comorbidity across neurodevelopmental and externalizing conditions. Polygenic risk scores (PRS) for ADHD have previously been shown to predict ADHD clinical case status and ADHD traits in the general population (Martin, Hamshere, Stergiakouli, O'Donovan, \&; Thapar, 2014; Stergiakouli et al., 2015). However, it is still unknown to what extent these genetic risk variants are disorder specific, and how they may influence related neurodevelopmental and externalizing traits. We calculated ADHD PRS for 13,471 children from the Child and Adolescent Twin Study in Sweden, using results from the latest iPSYCHsychiatric Genomics Consortium ADHD genome-wide association (GWAS) meta-analysis. We used confirmatory factor analysis and structural equation modelling to estimate the associations between ADHD PRS and neurodevelopmental and externalizing traits, whilst accounting for covariance across traits. ADHD PRS were significantly associated with elevated levels of trait inattention, impulsivity, autism, learning difficulties, oppositional-defiant and conduct problems. However, only unique associations with impulsivity and conduct problems remained after accounting for cross-trait covariance via a general latent factor, on which all symptoms loaded positively (loadings $=.31-.91, \mathrm{SD}=.004-.028$ ). ADHD PRS explained $0.01 \%(\beta=.10, \mathrm{p}=.000)$ of the variance in the latent general factor, $0.005 \%(\beta=.073, \mathrm{p}=.000)$ in impulsivity and $0.003 \%(\beta=.052, \mathrm{p}$ $=.035$ ) in conduct problems. We replicated these findings using ADHD PRS based on a GWAS of population ADHD traits. Our results suggest that common genetic variants associated with clinically diagnosed ADHD have pleiotropic effects on neurodevelopmental and externalizing traits in the general population, which indicate that these associations appear to be largely non-specific. Nonetheless, the ADHD PRS does not only reflect a general liability to childhood neurodevelopmental traits, but also seem to capture genetic risk variants with unique effects on externalizing traits. 


\section{A direct test of the diathesis-stress hypothesis for depression}

\section{Nick Martin, Queensland Institute of Medical Research, Australia}

The diathesis-stress theory for depression states that the effects of stress on the depression risk are dependent on the diathesis or vulnerability, implying multiplicative interactive effects on the liability scale. We used polygenic risk scores for major depressive disorder calculated from the results of the most recent analysis from the Psychiatric Genomics Consortium as a direct measure of the vulnerability for depression in a sample of 5221 individuals from 3083 families. In the same we also had measures of stressful life events and social support and a depression symptom score, as well as DSM-IV MDD diagnoses for most individuals. In order to estimate the variance in depression explained by the genetic vulnerability, the stressors and their interactions, we fitted linear mixed models controlling for relatedness for the whole sample as well as stratified by sex. We show a significant interaction of the polygenic risk scores with personal life events $(0.12 \%$ of variance explained, $p$-value $=0.0076)$ contributing positively to the risk of depression. Additionally, our results suggest possible differences in the aetiology of depression between women and men. In conclusion, our findings point to an extra risk for individuals with combined vulnerability and high number of reported personal life events beyond what would be expected from the additive contributions of these factors to the liability for depression, supporting the multiplicative diathesis-stress model for this disease.

\section{The Colorado adoption/twin study of lifespan behavioral development and cognitive aging (CATSLife): an introduction}

Chandra Reynolds, University of California, Riverside, United States; Robin Corley, University of Colorado Boulder, United States; John DeFries, University of Colorado Boulder, United States; Naomi Friedman, University of Colorado Boulder, United States; John Hewitt, University of Colorado Boulder, United States; Robert Plomin, King's College London, Institute of Psychiatry, Psychology \& Neuroscience, United Kingdom; Soo Rhee, University of Colorado Boulder, United States; Andrew Smolen, University of Colorado Boulder, United States; Michael Stallings, University of Colorado Boulder, United States; Sally Wadsworth, University of Colorado Boulder, United States

Cognitive development during infancy, early childhood and adolescence, coupled with health and activity patterns, may lay down crucial cognitive reserves uniquely salient to later cognitive functioning. However, relatively little is known about the developmental genetic and environmental etiologies of individual differences in rates of agerelated cognitive change from a long-term longitudinal perspective. The purpose of this presentation is to introduce the CATSLife study, and its overarching goal to examine cognitive trajectories and their moderators - spanning infancy through early adulthood - to evaluate pathways of resilience and vulnerability at the threshold of midadulthood. CATSLife brings together the Colorado Adoption Project and parallel Longitudinal Twin Study, to leverage early life assessments and design features. The primary aims are to: (a) assess individual differences in growth and maintenance of cognitive abilities approaching midlife; (b) evaluate physical and biochemical factors (e.g., blood pressure, serum lipids) and genetic factors (e.g., APOE, polygenic risk scores) that may be associated with sustaining cognitive performance; and (c) evaluate environmental factors from infancy through early adulthood that may decrease, sustain or even boost cognitive performance (e.g., geocoded neighborhood features). Our first 21 months of recruitment at ages 30-45 includes testing of 580 participants towards our target of 1600 . To date, serum lipids and brain-derived neurotrophic factor (bdnf) have been assayed for 415 samples. APOE genotyping has been completed on 464 CAP (274 probands, 190 siblings) and 894 LTS participants (480 MZ, 414 DZ) using newly collected and stored samples; chip genotyping is pending. Geocoding validation has recovered $85-87 \%$ exact matches to earliest historical addresses, and census blocks for $99 \%$ of the participants. We present study plans and initial results of new projects (e. g., cognition and associations with APOE, activity engagement, and neighborhood stress; subjective versus objective neighborhood assessments) in the context of a lifespan perspective on cognitive aging.

\section{Aggression and academic performance}

Eero Vuoksimaa, University of Jyvaskyla, Finland; Sebastian Lundström, University of Gothenburg, Sweden; Meike Bartels, Vrije Universiteit Amsterdam, Netherlands; Toos Van Beijsterveldt, Vrije Universiteit Amsterdam, Netherlands; Anne Hendriks, Vrije Universiteit Amsterdam, Netherlands; Eveline De Zeeuw, Vrije Universiteit Amsterdam, Netherlands; Kauko Heikkilä, University of Helsinki, Finland; Lea Pulkkinen, University of Jyvaskyla, Finland; Richard Rose, Indiana University, United States; Paul Lichtenstein, Karolinska Institutet, Sweden; Dorret Boomsma, Vrije Universiteit Amsterdam, Netherlands; Jaakko Kaprio, University of Helsinki, Finland

Introduction: A negative relationship between aggression and academic performance has been reported. Here, we assess the effects of age, of different measures of aggression, and of different raters on the association and then ask whether the association between aggression and academic performance can be confirmed in within-family comparisons.

Methods: We studied three twin cohorts participating in the ACTION consortium (Aggression in Children: Unraveling gene-environment interplay to inform Treatment and InterventiON strategies; Ćhttp:// www.action-euproject.eu/): FinnTwin12 (from Finland, N=3,122), CATSS (from Sweden, N=5,387) and NTR (from Netherlands, N= 10,430). Grade point average (GPA) at ages 12 (FinnTwin12; NTR), 14 (FinnTwin12) and 15 (CATSS) was used as a measure of academic performance. Aggression at ages 7 (NTR), 10 (NTR), 12 (NTR; FinnTwin12), 14 (FinnTwin12), and 15 (CATTS) was measured with three different instruments: Child Behavior Checklist in NTR, Multidimensional Peer Nomination Inventory in FinnTwin12, and Strengths and Difficulties questionnaire in CATSS. We investigated the association between aggression and GPA by conducting betweenfamily and within-family analyses in each cohort separately for different aggression raters (parent, teacher, self) at different ages.

Results: Between-family analyses indicated consistently significant negative associations between aggression and GPA across different ages, instruments and raters. In within-family analyses of all twins, the negative association between aggression and GPA was statistically significant in 13 out of 15 analyses. In separate analyses limited to $\mathrm{MZ}$ twins, most of the associations were non-significant. However, within-family analyses of DZ twins indicated more significant associations between aggression and GPA. Conclusions: There is a robust negative association between aggression and academic performance as measured with GPA. The lack of association in within-family analyses of MZ twins indicate that this relationship is due to shared genetic influences between childhood aggression and academic performance. 


\section{Long-term prenatal exposure to paracetamol is associated with DNA methylation differences in children diagnosed with ADHD}

Kristina Gervin, University of Oslo, Norway; Hedvig Nordeng, University of Oslo, Norway; Eivind Ystrom, Norwegian Institute of Public Health, Norway; Ted Reichborn-kjennerud, Norwegian Institute of Public Health, Norway; Robert Lyle, Oslo University Hospital, Norway

Epidemiological studies have shown that long-term exposure to paracetamol during pregnancy is associated with attention-deficit/ hyperactivity disorder (ADHD). The mechanism by which paracetamol may modulate the increased risk of developing ADHD is currently unknown. We have conducted an epigenome-wide association study and investigated whether prenatal exposure to paracetamol is associated with DNA methylation changes, and if such changes were associated with ADHD. The participants $(n=384)$ were selected from a large prospective birth cohort (Norwegian Mother and Child Cohort Study, MoBa), which contains information about medication use during pregnancy. The ADHD diagnoses were obtained from the Norwegian Patient Registry. Stratified analyses on ADHD and paracetamol separately did not identify any differentially methylation. However, comparison of samples with ADHD exposed to paracetamol for more than 20 days to healthy controls identified differentially methylated $\mathrm{CpGs}(\mathrm{n}=6211)$. In addition, these samples were differentially methylated compared to samples with ADHD exposed to paracetamol for less than 20 days ( $n=2089 \mathrm{CpGs}$ ) and not exposed to paracetamol ( $\mathrm{n}=193 \mathrm{CpGs})$. Interestingly, several of the top genes ranked according to significance and effect size have been linked to ADHD, neural development and neurotransmission. Gene ontology analysis revealed enrichment of pathways involved in oxidative stress, neurological processes and the olfactory sensory system, which have previously been implicated in the etiology of ADHD. These initial findings require replication, but suggest a possible mechanism by which prenatal long-term exposure to paracetamol modifies the risk of developing ADHD. Further, these results lend novel insights into the etiology of ADHD and may serve as disease biomarkers in blood.

\section{Stability and change in the etiology of educational achievement across school years}

Kaili Rimfeld, King's College London, Institute of Psychiatry, Psychology \& Neuroscience, United Kingdom; Margherita Malanchini, King's College London, Institute of Psychiatry, Psychology \& Neuroscience, United Kingdom; Robert Plomin, King's College London, Institute of Psychiatry, Psychology \& Neuroscience, United Kingdom

Individual differences in educational achievement are highly heritable $(60 \%)$. However, little is known about developmental change and continuity. Here, we study the etiology of educational achievement longitudinally across four ages from primary school (age 7) until the end of compulsory education (age 16) using both teacher ratings and exam scores from the UK representative Twins Early Development Study (TEDS) sample (N=6000 twin pairs). Genetic and environmental estimates were as expected (A 60\%, C 20\% and $\mathrm{E} \sim 20 \%$ ) with little change across the school years or across subjects. Educational achievement is highly stable across all four ages (phenotypic correlations of about 0.70$)$ and this stability is largely $(80 \%)$ explained by genetic factors, while non-shared environmental factors were mostly $(55 \%)$ age specific, contributing to change in educational achievement across the school years. About half of the phenotypic stability is explained by intelligence, but the etiology and stability of educational achievement did not change when controlling for intelligence. This pattern was found for overall achievement as well as for specific school subjects. Polygenic score analyses, using years of education (EduYears) summary statistics, indicated that EduYears explained more variance in overall achievement $(\sim 9 \%)$ compared to specific school subjects $(\sim 5 \%)$ at the end of the compulsory education. In addition, EduYears explained more variance in later $(\sim$ $9 \%)$ compared to earlier school years $(\sim 4 \%)$. We conclude that educational achievement throughout the school years is highly stable for all academic subjects and that this stability is driven genetically, even after controlling for intelligence. Educational achievement is an extreme example of the general behavioral genetic rubric of 'genetic stability, environmental change'. For this reason, adult EduYears predicts educational achievement even in the early school years.

\section{Does association between APOE e4 genotype and brain structure increase with older age in UK Biobank? $(\mathrm{N}=$ 1,217)}

Donald Lyall, University of Glasgow, United Kingdom, Simon Cox, University of Edinburgh, United Kingdom, Carlos Celis-Morales, University of Glasgow, United Kingdom, Daniel Mackay, University of Glasgow, United Kingdom, Rona Strawbridge, University of Glasgow, United Kingdom, Laura Pidgeon, University of Glasgow, United Kingdom, Breda Cullen, University of Glasgow, United Kingdom, Joey Ward, University of Glasgow, United Kingdom, Andrew Mcintosh, University of Edinburgh, United Kingdom, Daniel Smith, University of Edinburgh, United Kingdom, Ian Deary, University of Edinburgh, United Kingdom, Naveed Sattar, University of Glasgow, United Kingdom, Jill Pell, University of Glasgow, United Kingdom

The apolipoprotein e (APOE) e4 genotype is a known risk factor for cognitive decline and dementia, though the differences in brain anatomy which underpin this are unclear. The deleterious effects of this genotype on brain structure may increase in magnitude with increasing age. Structural magnetic resonance imaging of the brain, and genetic data, both from the general-population UK Biobank cohort $(\mathrm{N}=1,217)$ were used to test for association between APOE e4 allele presence (vs. absence) and brain structural imaging phenotypes, and to test whether there was significant interaction with cross-sectional age (at time of imaging). There was evidence of significant interaction between APOE e4 allele presence and increasing age on reduced white matter tract integrity, using tract fractional anisotropy as a proxy; where increased age was associated with less healthy white matter, and possessing an e4 allele exaggerated that association. These findings survived adjustments for age and sex, Townsend social deprivation scores, and cardiometabolic diseases, however did not survive false discovery rate correction. There were no interactions between e4 genotype and increasing age on: hippocampal volumes, total grey matter, or total white matter volume. Associations between APOE genotype and white matter integrity may be partly dependent on age at assessment, where there may be a modestly greater effect of e4 allele presence (vs. e4 absence) in older people. This may explain equivocal findings across different participant samples. 


\section{Results from a longitudinal twin study combining diagnostic interviews and registry data on personality, mental disorders, and substance use}

Fartein Ask, Torvik Norwe, Norway, Tom Rosenström, Norwegian Institute of Public Health, Norway; Nikolai Czajkowski, University of Oslo, Norway; Elizabeth Long, Virginia Commonwealth

University, United States; Nathan Gillespie, Virginia Commonwealth University, United States; Ted Reichborn-Kjennerud, Norwegian Institute of Public Health, Norway

The Norwegian Institute of Public Health Twin Panel has followed a population-based twin sample since 1998 with questionnaires and interviews and in governmental health registries. The diagnostic interviews include DSM criteria based measures (CIDI and SIDP) on internalizing and externalizing mental disorders, substance use and abuse and personality disorders. In addition, we have a rich set of questionnaires, covering normal personality, alternative conceptualization of malfunctioning personality, life events, and lifestyle. The registry data include measures of education, income, employment, disability, and primary and secondary health service use. In total, questionnaire and registry data are available for 11,727 individuals and interview data are available for 2,801 twins. We will present results from these rich data. The symposium covers methodological issues such as whether governmental health registries reflect the same etiological factors as mental disorders assessed at interview. We then proceed to discuss whether different conceptualization of personality and personality disorders measure the same etiological factors, how personality disorders relate to substance use, and how substance use relates to stressful life events and psychosocial outcomes.

\section{Genetic and environmental contribution to the overlap between ADHD and ASD dimensions in adults: a twin study}

Laura Ghirardi, Karolinska Institutet, Sweden; Erik Pettersson, Karolinska Institutet, Sweden; Paul Lichtenstein, Karolinska Institutet, Sweden; Henrik Larsson, Karolinska Institutet, Sweden; Ralf Kuja-Halkola Karolinska Institutet, Sweden

Introduction: Attention-deficit/hyperactivity disorder (ADHD) and autism spectrum disorder (ASD) frequently co-occur. One previous twin study in adults has found a er phenotypic and genetic overlap between traits of ADHD and traits of ASD related to repetitive and restrictive behaviours and interests (Polderman TJ, Hoekstra RA, Posthuma D, Larsson $\mathrm{H}$. The co-occurrence of autistic and ADHD dimensions in adults: an etiological study in 17,770 twins. Transl Psychiatry. 2014). To obtain more insight into the aetiology of the cooccurrence of these disorders in adulthood, we estimated the contribution of genetic and non-genetic effects to the association between ADHD traits and ASD traits in adults.

Methods: We use data from the Swedish Study of Young Adult Twins (YATSS) on 2122 twin pairs aged 20-28. Two dimensions of ADHD -inattention (IA) and hyperactivity/impulsivity (HI) - were assessed using the WHO Adult ADHD Self-Report Scale-V1.1. Two dimensions of ASD-repetitive and restrictive behaviours (RRB) and social interaction and communication (SIC)-were assessed using the Autism-Tics, AD/HD and other Comorbidities inventory. We used structural equation modelling to decompose co-variance between ADHD and ASD dimensions into genetic and environmental contribution and to estimate correlations between genetic and environmental effects acting on each ADHD and ASD dimension. The model was adjusted for age and sex.
Results: At the phenotypic level, IA was associated with both dimensions of ASD $(r=.36 ; r=.35)$, while HI was more highly associated with RRB ( $r=.40)$, than with SIC $(r=.24)$. Genetic and nonshared environmental effects accounted for a similar proportion of the phenotypic correlations. The highest genetic correlation was observed between HI and RRB (0.57), while the lowest was observed between $\mathrm{HI}$ and SIC (0.31).

Conclusions: The phenotypic and etiologic overlap between ADHD and ASD seems to be, at least partially, dimension-specific in adults. Both genetic and environmental effects seem to be important for such overlap.

\section{The relationship between maternal positivity and child positive affectivity: a cross-lagged analysis}

Dorothy White, The George Washington University, United States; Megan Flom, Boston University, United States; Julia Mancini, The George Washington University, United States; Jody Ganiban, The George Washington University, United States; Kimberly Saudino, Boston University, United States

Few studies have examined the relationship between maternal positivity (MP) and child positive affectivity (CP) across toddlerhood, and if it is best explained by genetic or environmental factors. The current study addressed this question by using a cross-lagged design to examine the stability, change, and reciprocal influences of these constructs from ages 2 to 3 years. Participants included 314 same-sex twin pairs from the Boston University Twin Project $\left(\mathrm{N}_{\mathrm{MZ}}=144, \mathrm{~N}_{\mathrm{DZ}}\right.$ $=168$ ) assessed at ages 2 and 3. CP was assessed via the Pleasure subscale of the Toddler Behavior Assessment Questionnaire (parent report). This subscale captures individual differences in the intensity of positive reactions to expected pleasurable activities. MP was assessed via self-report of feelings toward the child (Parent Feelings Questionnaire) and items from a parent discipline questionnaire. MP showed shared environmental influences at ages $2\left(C^{2}=.69\right)$ and $3\left(C^{2}\right.$ $=.58$ ). Genetic and nonshared environmental influences were observed at ages $2\left(\mathrm{~A}^{2}=.16 ; \mathrm{E}^{2}=.15\right)$ and $3\left(\mathrm{~A}^{2}=.18 ; \mathrm{E}^{2}=.21\right)$. $\mathrm{CP}$ showed significant shared and nonshared environmental influences at ages $2\left(C^{2}=.44 ; E^{2}=.17\right)$ and $3\left(C^{2}=.40 ; E 2=.35\right)$. Genetic influences on $\mathrm{CP}$ were significant at age $2\left(\mathrm{~A}^{2}=.39\right)$ and at $3\left(\mathrm{~A}^{2}=.25\right)$. The phenotypic associations between MP and $\mathrm{CP}$ at ages 2 and 3 were respectively $r=.28$ and $r=.31$. Both associations were explained by shared environmental factors. Both MP and CP were moderately stable over time $\left(\beta_{\mathrm{MP}}=.43, \beta_{\mathrm{CP}}=.52\right) . \mathrm{CP}$ at age 2 significantly predicted MP at age $3\left(\beta_{21}=.10\right)$, suggesting that CP affects MP over time. This association was primarily driven by shared environmental factors. These findings suggest that child positive affectivity does affect parental warmth and affection. Shared environmental influences may be driving this relationship. These influences could reflect a family-level affective style or climate that fosters positivity and warmth for mothers and children.

\section{Childhood aggression and adulthood adversities: general and specific associations}

Paul Lichtenstein, Karolinska Institutet, Sweden; Erik Pettersson, Karolinska Institutet, Sweden; Sebastian Lundström, University of Gothenburg, Sweden; Martin Cederlof, Karolinska Institutet, Sweden

Childhood aggression is associated with the development of externalizing problems in adulthood (e.g., criminality, substance misuse). 
A few studies have found that aggressive children develop also other types of problem, but it is unknown whether childhood aggression is a non-specific predictor of adult adversities or whether it is specific for externalizing behaviors. This prospective twin study is based on a telephone interview with parents to 18,649 9/12 year old twins from the Child \& Adolescent Twin Study in Sweden (CATSS). Childhood aggression was assessed with eight items. Outcomes at age 15-21 (severe mental illness, depression, anxiety, anti-depressant/anxiolytic medications, suicide attempts, alcohol/substance use disorders, school failure, social welfare, criminality) were ascertained through national health, demographic and crime registers. Childhood aggression predicted all the adversities in young adulthood. The outcomes clustered into one general (including all outcomes), one internalizing, and one externalizing factor. Aggression had substantial associations with all factors (general: $\beta=0.43$; externalizing: $\beta=0.43$; internalizing: $\beta=$ $0.35)$. Genes contributed to the association between childhood aggression and the general $(\beta=0.35)$ and internalizing $(\beta=0.33)$ factors, whereas both genes $(\beta=0.23)$ and shared environments $(\beta=0.41)$ contributed to the association with the specific externalizing factor. Childhood aggression is associated with all types of adversities in adolescence/young adulthood and not just externalizing outcomes. Whereas genetic effects are important for the development into all types of adulthood adversities, shared environmental effects seem to be important specifically for developing externalizing problems. This suggests that interventions for aggressive children should depend on whether the aim is to prevent externalizing or other types of adulthood adversities.

\section{Parenting quality and mathematics performance in early childhood: using an adoption design to disentangle genetic and environmental}

Giulia Borriello, The Pennsylvania State University, United States; Amanda Ramos, The Pennsylvania State University, United States; Leslie Leve, University of Oregon, United States; Misaki Natsuaki, University of California, Riverside, United States; David Reiss, Yale University, United States; Daniel Shaw, University of Pittsburgh, United States; Jenae Neiderhiser, The Pennsylvania State University, United States

Early mathematics knowledge is predictive of later mathematical abilities. Supportive parenting behaviors (e.g., maternal warmth) have been associated with children's math performance, although few studies have examined these associations using genetically-informed designs. Here, we used data from Cohort I of the Early Growth and Development Study, a parent-offspring adoption sample $(\mathrm{N}=361)$, to examine the potential moderation of adoptive parent (AP) supportive parenting (e.g., positive reinforcement) and hostile parenting on associations between birth parent (BP) math performance and child math performance during middle childhood. BP and child math performance (at age 7) was assessed using the mathematics fluency measure of the Woodcock-Johnson III Achievement Test. APs reported on their supportive parenting using the Alabama Parenting Questionnaire and hostile parenting using the IOWA Family Interaction Rating Scales at child age $41 / 2$ years. Preliminary findings indicate direct effects of BP math fluency on child math fluency, but no direct effects of AP support and hostility on child math fluency. We found significant moderation of adoptive mothers' supportive parenting $(\beta=.23, \mathrm{t}=3.53, \mathrm{p}<.01)$ and hostility $(\beta=-0.18, \mathrm{t}=-2.54, \mathrm{p}$ $=.01$ ) on child math fluency. Simple slope analyses reveal that in cases where the adopted child experienced high levels (but not low) of support, high BP math fluency (heritable influences) was related to high child math performance, $(\beta=.46, t=4.63, p<.001)$. Similarly, for children who experienced lower levels of maternal hostility (but not high), high BP math fluency was related to high child math performance $(\beta=.37, \mathrm{t}=3.65, \mathrm{p}<.001)$. These findings suggest that heritable influences on children's mathematics performance emerge when the child's rearing environment provides heightened maternal support.

\section{Sibling comparisons of polygenic scores: evidence for quasi-causal effects of educational attainment polygenes on substance use disorders}

Jessica Salvatore, Virginia Commonwealth University, United States; Fazil Aliev, Virginia Commonwealth University, United States; Peter Barr, Virginia Commonwealth University, United States; Sally Kuo, Virginia Commonwealth University, United States; inni Su, Virginia Commonwealth University, United States

Low educational attainment is associated with higher rates of substance use disorder (SUD) diagnoses. We took a genetically informed perspective to understand the nature of these associations and examined whether (1) genome-wide polygenic scores for educational attainment (EDU-GPS) also predict SUDs (i.e., pleiotropic effects), and (2) whether the associations between EDU-GPS and SUDs are likely to be "causal". Our sample included 864 families of EuropeanAmerican siblings who were 25 years of age or older (2-12 siblings per family; 2317 total individuals; $53 \%$ female) collected as part of the Collaborative Study on the Genetics of Alcoholism (COGA). Our SUD outcomes included lifetime criterion counts for DSM-5 Alcohol Use Disorder (AUDSX); Cannabis Use Disorder (CUDSX); and Fagerstrom Nicotine Dependence (NDSX). Educational attainment polygenic scores (EDU-GPS) were calculated in the COGA sample using genome-wide association weights from the Social Science Genetic Association Consortium. We used mixed models to examine whether EDU-GPS was associated with the three SUD criterion counts at the population level. Covariates included age; cohort; sex; the number of variants available for polygenic scoring; and two genetic ancestry principal components. We found that higher EDUGPS predicted lower AUDSX, CUDSX, and NDSX (ps<0.01). We then used sibling comparisons to examine whether the populationlevel associations between EDU-GPS and SUDs were likely to be "causal". Sibling comparisons control for the other genetic and environmental background factors that siblings share, and thus permit a quasi-causal test of the effects of EDU-GPS on SUDs. Those with higher EDU-GPS compared to their siblings met fewer SUD criteria, although the effect for AUDSX was only at the trend level (AUDSX: $\mathrm{b}=-5.84, \mathrm{p}=.06$; CUDSX: $\mathrm{b}=-119.81, \mathrm{p}<.01$; and NDSX: $\mathrm{b}=-6.65$, $\mathrm{p}=.03$ ). This suggests that EDU-GPS may have a causal, protective effect against the development of SUDs.

\section{Etiological (dis)similarity between individual alcohol use disorder symptoms}

\section{Arielle Deutsch, Pennsylvania State University, United States}

Previous literature indicates that overall heritability of alcohol use disorder (AUD) is approximately 50\%. However, there is little research examining etiological similarity between individual AUD symptoms or hazardous drinking behavior. Waves 1 and 3 of the National Longitudinal Study for Adolescent to Adult Health (Add Health) sibling subsample [319 MZ pairs (145 male; 144 female) and 476 DZ pairs (117 male; 131 female; 204 opposite)] were analyzed. Past-year endorsement of outcomes related to three AUD symptoms were assessed: hazardous behavior ([a] fighting or [b] having 
regretted sex while drinking), interference (having trouble with [a] school or work, [b] romantic partners, or [c] friends), and sickness/ aftereffects ([a] vomiting or [b] hangovers). Past-year risky drinking based on the NIAAA guidelines was also assessed: drinking more than 7/14 drinks per week (for women and men respectively) and no binge drinking. Waves 1 and 3 were assessed to examine both adolescent (W1 age $\mathrm{M}=15.65, \mathrm{SD}=1.74$ ) and young adult (W3 age $\mathrm{M}=$ $21.89, \mathrm{SD}=1.75$ ) etiology, as well as stability across time. Univariate biometric models indicated that heritability varied across outcomes. Percentage attributed to additive genetic variance ranged from $0 \%$ (trouble with school/work; fighting) to $60 \%$ (having regrettable sex) in adolescence, and from $0 \%$ (fighting) to $36 \%$ (vomiting) in young adulthood. Variance was typically non-significant across all outcomes at both age periods. Variance attributed to unique environment in young adulthood was the most consistent factor, ranging from $43 \%$ (trouble at school/work) to $73 \%$ (having regrettable sex). Unique environment factors were typically significant in adolescence and young adulthood. Bivariate biometric analyses were then estimated in which all outcomes were paired with each other individually and with risky drinking, at each age period (i.e., 28 models per wave), as well as biometric models that analyzed similarity over waves. Results indicated a range of $\mathrm{rG}, \mathrm{rC}$, and $\mathrm{rE}$ scores, highlighting the variability in etiological similarity across symptoms, and in relation to risky drinking behavior itself. There was also little stability in relations between additive genetic and environmental variance over adolescence and young adulthood for symptoms over adolescence and young adulthood.

\section{Sleep problems from 18 months to 6 years: a mechanism in the intergenerational transmission of psychopathology?}

Kimberly Rhoades, Washington State University, United States; Leslie Leve, University of Oregon, United States; Misaki Natsuaki, University of California, Riverside, United States; Jenae Neiderhiser, The Pennsylvania State University, United States; Daniel Shaw, University of Pittsburgh, United States; Jody Ganiban, The George Washington University, United States; David Reiss, Yale University, United States

Parental psychopathology is a robust predictor of psychopathology in children and has been shown to operate via both genetic and environmental pathways. Childhood sleep problems may serve a mediating role in these pathways via bioregulatory mechanisms. Although parental psychopathology is associated with child sleep problems and psychopathology, it is unclear whether sleep problems serve as a mechanism in the intergenerational transmission of psychopathology. We examined the potential mediating role of child sleep problems from 18 months to 6 years of age on associations between birth parent and adoptive parent depressive symptoms and child emotional and behavioral disorders in a longitudinal prospective parent-offspring adoption sample ( $\mathrm{N}=561$ linked triads). We included parental depressive symptoms due to the genetic overlap between depressive symptoms and sleep problems and environmental associations between parental depression and child sleep problems. We examined: (1) associations between birth and adoptive parent depressive symptoms and child emotional and behavioral disorders; and (2) whether sleep problems mediated associations between depressive symptoms and child emotional and disruptive behavior disorders. Analyses identified significant associations between birth mothers' depressive symptoms and the number of child emotional and disruptive behavior disorders diagnosed on the PAPA at age 6 (cohort
2) or 8 (cohort 1$)(\beta=.11 ; \mathrm{p}<.05)$. Adoptive mother depressive symptoms significantly predicted child sleep problems $(\beta=.20$; $\mathrm{p}<.001)$ and sleep problems significantly predicted the number of child emotional and disruptive behavior disorders $(\beta=.36 ; \mathrm{p}<.001)$. The indirect effect between adoptive mother depressive symptoms and child emotional and disruptive behavior disorders via child sleep was significant $(\beta=.07 ; \mathrm{p}<.05)$. Thus, birth mothers' depressive symptoms were directly associated with child emotional and behavioral disorders whereas adoptive mothers' depressive symptoms were indirectly associated with child emotional and behavioral disorders via child sleep problems.

\section{Genetic correlation between nausea and vomiting during pregnancy and major depressive disorder}

Lucia Colodro Conde, QIMR, Australia; Lavinia Paternoster, University of Bristol, United Kingdom; Penelope Lind, QIMR, Berghofer Medical Research Institute, Australia; Jodie Painter, QIMR, Berghofer Medical Research Institute, Australia; Margaret Wright, The University of Queensland, Australia; Grant Montgomery, The University of Queensland, Australia; Nicholas Martin, QIMR, Australia; Dale Nyholt, Queensland University of Technology, Australia; Sarah Medland, QIMR, Berghofer Medical Research Institute, Australia

Nausea and vomiting in pregnancy (NVP) affects $\sim 70 \%$ of pregnant women to different degrees. Historically, the dominant aetiological theory of these conditions was psychogenic in origin, being commonly described as a conversion disorder. Recent studies have shown that women who experience severe NVP have higher rates of major depressive disorder (MDD) prior to the index pregnancy. NVP and depression are significantly heritable. We therefore hypothesize that there is a genetic correlation between NVP and depression. We first carried out a genomewide association study (GWAS) meta-analysis on the presence of NVP with the summary statistics provided by two samples which are part of the NVP Genetics Consortium: QIMR Berghofer Medical Research Institute $(n=1441)$ and the Avon Longitudinal Study of Parents and Children $(n=7053)$. The GWAS analyses included age and age squared at time of interview, singleton/ twin pregnancy variables, and the first four ancestry principal components as covariates. Both autosomal and $\mathrm{X}$ chromosomes were analysed and genotypes were analysed as allele doses. Meta-analysis of summary statistics of the two samples was carried out with METAL simultaneously. We conducted a linkage disequilibrium score regression with the PGC-MDD results to calculate the genetic correlation of both traits using SECA (SNP effect concordance analysis using genome-wide association summary results, Nyholt 2014). The GWAS meta-analysis did not show any genome-wide significant hit. The SNP with the lowest p-value (rs2238144, p= $1.237 \mathrm{e}-07$ ) is located within the RAPGEF3 gene (Rap guanine nucleotide exchange Factor GEF 3) in a DNAse hypersensitivity region on chromosome 12 , with signalling pathway participating in the stimulation of the placental cell fusion. There was a significant concordance of genetic risk between NVP and MDD, with allelic effects that increase risk for both traits. The implications of these results for the treatment of depression and anxiety with severe NVP will be discussed.

\section{References}

Nyholt D.R. (2014). SECA: SNP effect concordance analysis using genome-wide association summary results. Bioinformatics 30, 2086-2088. 


\section{The challenge of genetics to social inequality research}

\author{
Martin Diewald, Bielefeld University, Germany
}

In the social sciences the long-standing reluctance against genetically informative studies has diminished, and overcoming the traditional "biophobia", interest has grown in the varieties of how and when genetic and social influences interfere to shape different life chances over the life course. Recognising the roles of genetic factors in producing social inequality is important for methodological and substantial reasons. Purely social investigations of family-of-origin influences obscure the statistical confounding that genetic transmission introduces. However, acknowledging the role genes does not at all mean to deny or even to relativize the role of social forces. Quite in contrast, the last years of genetically informed research made the paramount importance of social forces to understand genetic expression more evident. I discuss this along some promising questions for future research where social scientists could profit a lot from genetic information and vice versa geneticists for mechanisms of genetic expression.

(1) The role of individual agency and exogeneity of environmental influences over the life course: ACE decomposition methods, including rGE, allow for a more comprehensive understanding of one of the core questions of social science research, namely the relation between structuration and agency.

(2) The interplay between genetic and social forces structuring the puzzle of the intergenerational transmission of advantage and disadvantage: Against the too simple of notion of genes influencing social outcomes via psychological characteristics, the role of genetic and social forces, and the degree to which social forces are shared environment, varies a lot between different social transitions and individual development of cognitive and non-cognitive skills, with changing patterns over individual lifetime. For these two aspects first results based on the recently launched German TWINLIFE data are presented. However, for disentangling obvious contradictions between commonly modest to null shared environment estimates in BG research (except education) versus the sociological focus on the importance of parental resources and behaviors, there is still a lot to be done.

A third important aspect is a move from almost exclusive interest in proximal to more research on the role of distal environments: there is definitely a need for systematic international comparisons in $B G$ research.

\section{Phenotypic and genetic correlations between diagnosed neurodevelopmental and internalizing disorders}

Ralf Kuja-Halkola, Karolinska Institutet, Sweden; Isabell Brikell, Karolinska Institutet, Sweden; Laura Ghirardi, Karolinska Institutet, Sweden; Qi Chen, Karolinska Institutet, Sweden; Paul Lichtenstein, Karolinska Institutet, Sweden; Henrik Larsson, Karolinska Institutet, Sweden

Backgound: Neurodevelopmental disorders (NDDs) such as ADHD, Intellectual Disability (ID), and Autism Spectrum Disorders (ASD), with onset in childhood, have been thoroughly researched with regards to phenotypic and genetic overlaps. Similarly, internalizing disorders such as anxiety, depression, and phobias, with onset in adolescence/adulthood, although not as thoroughly, has been assessed for overlaps. Research efforts have been made both for symptomlevel data and diagnoses within the disorder groups. Although the disorder spectrums are associated, e.g. by NDDs later developing into internalizing disorders, to our knowledge, no previous study has used family-relation data to specifically investigate the phenotypic, genetic, and environmental overlap between NDD and internalizing disorder spectrums.

Therefore, we estimated the phenotypic and genetic associations between NDDs (ADHD, ASD and ID) and internalizing disorders (anxiety, depression, and phobias) using clinical diagnoses and familial relations in the Swedish population.

Method: In a cohort born 1982 through $1996(\mathrm{~N}=1,456,683)$ we identified individuals with and without observed diagnoses in the Swedish National Patient Register. We used the Swedish Twin Register and Multi-Generation Register to identify siblings (MZ and DZ twins, full- and half-siblings). Using the liability-threshold model we then proceeded to estimate the phenotypic (tetrachoric) correlation between all disorders, and the genetic and environmental correlations between all disorders.

Results: The phenotypic correlations, within individuals between disorders, ranged between 0.47 and 0.64 within NDDs and between 0.64 and 0.74 within internalizing disorders. Between NDDs and internalizing disorders correlations ranged from 0.15 to 0.52 . Additive genetics explained between 57 and $83 \%$ of the correlations between NDDs, between 33 and 55\% between internalizing disorders, and between 60 and $100 \%$ between NDDs and internalizing disorders. Additive genetic correlations were weaker, 0.53-0.69, within NDDs than within internalizing disorders, $0.87-1.00$, and similar in strength between internalizing disorders and NDDs as within NDDs, 0.240.85 .

\section{Maternal smoking during pregnancy does not affect externalizing problem behavior in children}

Tinca Polderman, Vrije Universiteit Amsterdam, Netherlands; Philip Jansen Vrije, Universiteit Amsterdam, Complex Trait Genetics, Center for Neuroscience and Cognitive Research; Department of Child and Adolescent Psychiatry, Erasmus Medical Center, Rotterdam, The Netherlands, Netherlands; Jorim Tielbeek, Vrije Universiteit Amsterdam, Complex Trait Genetics, Center for Neuroscience and Cognitive Research; Department Child and Adolescent Psychiatry, VU University Medical Center Amsterdam, The Netherlands, Netherlands; Henning Tiemeier, Erasmus Medical Center, Netherlands; Hadi Zafarmand, Universiteit van Amsterdam, Amsterdam Medical Center, Dept. Public Health, Netherlands; Tanja Vrijkotte, Universiteit van Amsterdam, Amsterdam Medical Center, Dept. Public Health, Netherlands

Externalizing problem behaviours are associated with negative longterm outcomes such as antisocial behaviour, substance abuse, and socioeconomic problems. Externalizing behaviour is heritable $(\sim$ $50 \%$ ) but is also associated with environmental risk factors such as maternal smoking during pregnancy (MSDP). A crucial question is: do environmental risk and genetic factors pose an independent effect on externalizing problems, or do they interact? We tested in two large samples (ABCD; $N=1,154$ and Generation $\mathrm{R}, \mathrm{N}=2,200$ ) the effects of MSDP and genetic risk on externalizing behavioural problems. MSDP was derived from mother reports, or interviews. Externalizing problems in ABCD were assessed with mother and teacher reports at age 5 , and with mother and self reports at age 12 using the Strengths and Difficulties Questionnaire (SDQ). In Generation R, the Child Behavior Checklist was assessed at age 5 and 9 with mother reports. Genetic risk was based on polygenic risk scores (PRS) derived from the largest GWAS to date on antisocial behaviour, (BroadABC N= $16,400)$ and on ADHD $(\mathrm{N}=55,374)$. Our model included PRS of antisocial behaviour or ADHD, MSDP, sex, and educational attainment of the mother. We observed no main effect of antisocial PRS on 
externalizing problems in both samples. We did observe a significant positive main effect of MSDP on mother reported externalizing problems, and an interaction effect with antisocial PRS, but only at age 5, and only in ABCD. The ADHD PRS was significantly associated with externalizing problems in both samples, at both ages, but we found no significant effects of MSDP. Our observations do not support a main or interaction effect of MSDP on externalizing problem behaviors in young children. Future studies should include genetic risk when exploring environmental risk factors for psychopathological problems, such as externalizing problem behaviors.

\section{The role of cultural conditions: genetic influences on alcohol misuse across adolescence and early adulthood in Germany and the US}

Elisabeth Hahn, Saarland University, Germany; Juliana Gottschling, Saarland University, Germany; Frank M. Spinath, Saarland University, Germany; Matt McGue, University of Minnesota, United States; Wendy Johnson, University of Edinburgh, United Kingdom

During lifetime, alcohol misuse can cause serious problems including physical, social, and economic complications. In the last decade, potential biological and social markers of risk have been identified indicating that both genetic and environmental influences play a role. However, the importance of genetic influences on alcohol misuse can also vary as a function of environmental conditions. So far, knowledge is restricted with respect to the question when and how the society with its restrictions on alcohol consumption probably interacts with the persons' individual genetic makeup. In the current study, we investigated genetic and environmental factors on the etiology of problematic alcohol consumption including drinking initiation, regular and problematic drinking in countries with differing rules regarding alcohol consumption, i.e. the US and Germany. We compared mean levels and variances as well as genetic and environmental influences on problematic alcohol consumption for samples of twins and their parents drawn from the German twin family study TwinLife $(\mathrm{N}=3.078$ twin families) and the Minnesota Twin Family Study ( $\mathrm{N}=4.153$ twin families). Each study clustered twins into three age cohorts $(11,17$ and $23 / 24$ years) to investigate developmental trajectories. The results showed substantial mean differences between the countries for regular and problematic drinking especially at age of 23/24 as well as consistent sex-effects in both countries across ages. Regarding the importance of genetic influences on drinking behavior, analyses showed differing results depending on the respective age group. Findings and implications will be discussed with respect to the role of cultural conditions.

\section{Genetic evidence for psychiatric disorders as the extreme end of a continuum of population traits}

Joanna Martin, Karolinska Institutet, Sweden; Mark Taylor, Karolinska Institutet, Sweden; Lu Yi, Karolinska Institutet, Sweden; Isabell Brikell, Karolinska Institutet, Sweden; Sebastian Lundström, University of Gothenburg, Sweden; Paul Lichtenstein, Karolinska Institutet, Sweden

Psychiatric disorders are highly heritable and polygenic, with numerous common genetic risk variants implicated. Twin and molecular genetic studies suggest that some clinically diagnosed psychiatric disorders (e.g. attention-deficit/hyperactivity disorder (ADHD) or autism) are also linked with quantitative variation in milder traits of the same disorder throughout the general population. We utilized the latest emerging results from genome-wide association studies (GWAS) of 8 psychiatric disorders to test for genetic overlap across a range of clinically diagnosed psychiatric disorders and related continuously distributed population traits.

GWAS summary statistics for 8 psychiatric phenotypes were used to derive polygenic risk scores (PRS) in a Swedish population of twins ( $\mathrm{N}=13,472$ individuals) with data available at ages 9, 12, 15 and 18 years (Child Adolescent Twin Study in Sweden). We tested for association between PRS for a given psychiatric disorder with traits related to the same disorder, assessed using parent- and self-report questionnaires. After applying a false discovery rate to account for multiple testing, the results show support for a significant association between PRS for psychiatric disorder with corresponding childhood population traits for the following phenotypes: ADHD (beta $(\mathrm{SE})=$ $0.278(0.0295), p=6.8 \mathrm{E}-20)$, autism (beta $(\mathrm{SE})=0.0366(0.0137), \mathrm{p}$ $=.019)$, tic disorders (beta $(\mathrm{SE})=0.0126$ (0.00387), $\mathrm{p}=.0063)$, depression (beta $(\mathrm{SE})=0.273(0.105), \mathrm{p}=.021)$, anxiety disorders (beta $(\mathrm{SE})=0.202 \quad(0.0795), \quad \mathrm{p}=.023), \quad$ obsessive-compulsive disorder $(\mathrm{OCD}$; beta $(\mathrm{SE})=0.122(0.0447), \mathrm{p}=.019)$ and schizophrenia (beta $(\mathrm{SE})=0.0561(0.0208), \mathrm{p}=.019)$. No association is seen for bipolar disorder PRS and adolescent mania symptoms. Results persisted after excluding individuals with relevant ICD diagnoses from analyses.

This study provides evidence for shared genetic risks across clinical disorder and corresponding population traits assessed in childhood, for a number of psychiatric conditions. The results support the hypothesis that psychiatric disorders are the extreme ends of continuous distributions of population traits.

\section{Epigenome-wide association study meta-analysis of aggressive behavior}

Jenny van Dongen, VU Amsterdam, Netherlands; Matthew Suderman, University of Bristol, United Kingdom; Karen Sugden, Duke University, United States; Khadeeja Ismail, University of Helsinki, Finland; Rosa H. Mulder, Universiteit Leiden, Netherlands; Leanne K. Küpers, University of Bristol, United Kingdom; Mariona Bustamante, ISGlobal, Center for Research in Environmental Epidemiology (CREAL), Spain; Silvia Alemany ISGlobal, Center for Research in Environmental Epidemiology (CREAL), Spain; Marc Jan Bonder, University Medical Centre Groningen, Netherlands; Priyanka Parmar, University of Oulu, Finland; Estelle Lowry, University of Oulu, Finland; Rick Jansen, VU University Medical Centre, Netherlands; Bart Baselmans, Vrije Universiteit Amsterdam, Netherlands; Michel Nivard, Vrije Universiteit Amsterdam, Netherlands; David Corcoran, Duke University, United States; Richie Poulton, University of Otago, New Zealand; Jon Mill, University of Exeter, United Kingdom; Eilis Hannon, University of Exeter, United Kingdom; Louise Arseneault, King's College London, United Kingdom; Eero Vuoksimaa, University of Oulu, Finland; Miina Ollikainen, University of Helsinki, Finland; Eva Corpeleijn, University Medical Centre Groningen, Netherlands; Lannie Ligthart, Vrije Universiteit Amsterdam, Netherlands; BIOS Consortium, NA, Netherlands; Caroline Relton, University of Bristol, United Kingdom; Terrie Moffitt, Duke University, United States; Avshalom Caspi, Duke University, United States; Jaakko Kaprio, University of Helsinki, Finland; Henning Tiemeier, Erasmus Medical Center, Netherlands; Harold Snieder, University Medical Centre Groningen, Netherlands; Jordi Sunyer, ISGlobal, Center for Research in Environmental Epidemiology (CREAL), Spain; Lude Franke, University Medical Centre Groningen, Netherlands; Sylvain Sebert, University of Oulu, Finland; Marjo-riitta, Jarvelin Imperial College, London, United Kingdom; Meike Bartels, Vrije Universiteit Amsterdam, Netherlands; Dorret Boomsma, Vrije Universiteit Amsterdam, Netherlands 
Aggressive behavior emerges early in childhood, peaking between the age of two and four, which is considered to be a normal aspect of development (Alink et al. 2006; Cote et al. 2006). Thereafter, aggression decreases in most children, along with the development of self-control and the learning of socially-preferred behavior. Adverse early life conditions, including low parental income, maternal smoking during pregnancy, and family dysfunction are associated with a higher level of aggressive behavior in childhood and with increased odds that a person continues on a chronic physical aggression trajectory in adolescence (Tremblay et al. 2004). Twin studies highlight the importance of genetic variation: heritability estimates of aggression range between 50 and $80 \%$ in children and adults (Porsch et al. 2016; Yeh et al. 2010). Life conditions may impact on genetic pathways involved in aggressive behavior by inducing changes to epigenetic modifications, such as DNA methylation, that regulate gene expression (Provencal et al. 2015). As part of the ACTION project (Aggression in Children: Unraveling geneenvironment interplay to inform Treatment and InterventiON strategies; http://www.action-euproject.eu/) we are performing an EWAS meta-analysis (EWAMA) of aggressive behavior and genome-wide DNA methylation assessed with the Illumina $450 \mathrm{k}$ array. This EWAMA includes 11 cohorts (total sample size $=9389$ ) with data for 8170 peripheral blood samples (mean age at blood sampling ranging from 4 to 44 ) and 2195 cord blood samples. The mean age at phenotypic assessment ranges from 6 to 44 and multiple instruments were used to assess aggressive behavior or conduct problems, such as the Child Behavior Check List (CBCL), Strength and Difficulty Questionnaire (SDQ), Multidimensional Peer Nomination Inventory (MNPI), ASEBA adult self-report (ASR), DSM Conduct Disorder Symptom Scale, Multidimensional Personality Questionnaire (MPQ), Temperament and Character Inventory (TCI), and the NEO personality questionnaire. Results of the meta-analysis will be presented at BGA.

\section{Correspondence between common mental disorders assessed in diagnostic interviews and health registries: implications for genetic studies}

Fartein Ask Torvik, Norwegian Institute for Public Health, Norway; Eivind Ystrom, Norwegian Institute of Public Health, Norway; Kristin Gustavson, Norwegian Institute of Public Health, Norway; Tom Rosenström, Norwegian Institute of Public Health, Norway; Kristian Tambs, Norwegian Institute of Public Health, Norway; Jørgen Bramness, University of Oslo, Norway; Nathan Gillespie, Virginia Commonwealth University, United States; Steven Aggen Virginia, Commonwealth University, United States; Kenneth Kendler, Virginia Commonwealth University, United States; Ted Reichborn-Kjennerud, Norwegian Institute of Public Health, Norway

Background: An increasing number of studies use administrative health registries. It is unknown to what extent findings on common mental disorders are generalizable to the population at large. We study the correspondence between structured interviews of a population-based twin sample and primary and specialist health care registries for major depressive disorder (MDD), anxiety disorders (AD) and alcohol use disorder (AUD) with regard to prevalence, comorbidity and genetic influences.

Methods: Registry data from primary and specialist care were available for 11,727 twins and diagnostic interview data for 2,271 of these. We used logistic regression analyses and biometric twin modelling to investigate the diagnostic overlap between interview, primary care and specialist care.

Results: Most individuals meeting criteria for common mental disorders according to interview data did not have a corresponding diagnosis in the health registries. The rates of registration were higher for MDD (36\% in primary care and $15 \%$ in specialist care) and AD (21 and 18\%) than for AUD (3 and 7\%). Comorbidity estimates were higher in the registries than in the general population. Genetic factors for the disorders were highly correlated across data sources (median $r$ $=.81$ ) and close to unity for MDD and AD.

Conclusion: Only a subset of individuals with common mental disorders as assessed at interview receive treatment. Research relying on data from health registries may substantially underestimate true prevalence and overestimate comorbidity. Diagnoses based on health registries reflect the same genetic influences as common mental disorders in the general population, indicating generalizability across data sources.

\section{New developments in the genetics of psychiatric comorbidity}

Elliot Tucker-Drob, University of Texas, United States; Paige Harden, University of Texas at Austin, United States; Irwin Waldman, Emory University United States; Adela-Maria Isvoranu, University of Amsterdam, Netherlands

A growing literature indicates that, in late childhood, adolescence and adulthood, genetic liabilities for psychopathology are largely nonspecific and that a general factor of psychopathology ("p") is negatively associated with intelligence. This symposium brings together four papers that seek to further elucidate these patterns. Harden presents results of an analysis of nearly 2000 twins ages 818 years from the Texas Twin Project, reporting cross-cutting associations between low intelligence and executive functioning, on the one hand, and a range of psychiatric symptomologies on the other hand. These associations are primarily mediated by shared genetic etiology between a general dimension of cognitive function and a general factor of psychopathology. Tucker-Drob examines the $g$ factor $-p$ factor association in the first 6 years of life. He uses integrative data analysis to examine genetic and environmental links between psychopathology and cognitive ability in the Texas "tiny" Twin Project and the twin subsample of the Early Childhood Longitudinal Study-Birth Cohort. Waldman uses both simulation and analysis of empirical data from approximately 1,600 twins ages 917 years to examine the robustness of the General Factor of Psychopathology and its external correlates to model specification. Finally, Isvoranu provides an overview of how genetic risk scores can be incorporated into formal graph theoretic model of symptom networks to represent how genetic influences on psychiatric symptom comorbidity might arise dynamically over time.

\section{Genetic and environmental links between general factors of psychopathology and cognitive ability in early childhood}

Elliot Tucker-Drob, University of Texas, United States; Paige Harden, University of Texas at Austin, United States; Amanda Cheung, University of Texas at Austin, United States; Andrew Grotzinger, University of Texas at Austin, United States; Megan Patterson, University of Texas at Austin, United States

Background: Recent work in adolescent and adult populations indicates that the comorbidity between a diverse range of psychiatric disorders and symptomologies is largely attributable to the operation of non-specific genetic factors that confer liability for a broad range of psychiatric symptoms, and are themselves inversely correlated with 
genetic influences on cognitive functioning. Although pairwise associations between specific markers of cognitive development and psychopathology have been reported in very early childhood, psychopathology comorbidity, its etiology, and its relations with general cognitive function in early development have gone unstudied.

Methods: We synthesized individual level data from two American twin studies of early child development: the Texas "Tiny" Twin project (age range $=0-5$ years, $\mathrm{N}=626$ twins, measured up to 11 occasions, for a total of 1,398 observations), and the twin subsample from the Early Childhood Longitudinal Study-Birth Cohort (age range $=3-7$ years; $N=\sim 1,300$ twins, measured up to 3 occasions for a total of $\sim 2,700$ observations), each of which provided parent report measures of internalizing, externalizing, and attentional/self-regulatory problems, and included five measures of cognitive and psychomotor development. We fit confirmatory factor models to test the dimensionality of the 8 phenotypes and, using an integrative data analysis approach, went on to estimate multivariate biometric models of the associations between abilities and psychopathology.

Results: Confirmatory factor models indicated that two factor solutions fit the data well, with the internalizing and externalizing symptoms loading on a general psychopathology (p) factor, the five cognitive psychomotor measures loading on a general ability ( $\mathrm{g}$ ) factor, and attention/self-regulatory problems loading on both $\mathrm{p}$ and $\mathrm{g}$. These general factors were moderately correlated in both samples ( $\mathrm{r}=$ -.21 and -.34). Biometric analyses indicated that genetic and environmental influences on $\mathrm{p}$ and $\mathrm{g}$ were moderately correlated, and that the total correlation between these factors was largely due to overlapping genetic and shared environmental influences.

Conclusions: General psychopathology in early childhood is negatively associated with general cognitive development. The current findings indicate this inverse association is attributable to common genetic and shared environmental factors.

\section{Socioeconomic status and heritability of cognitive performance in the Louisville Twin study}

Evan Giangrande, University of Virginia, United States; Lucas Matthews, University of Virginia, United States; Sarah Carroll, University of Virginia, United States; Eric Turkheimer, University of Virginia, United States

Turkheimer et al. (2003) found that heritability of IQ varies as a function of socioeconomic status (SES) in 7 year-old children, with children from impoverished families showing reduced heritability compared to affluent peers. The current study extended this line of work by examining interactions of SES and IQ heritability in children of other ages, using data from the recently resurrected Louisville Twin Study (LTS). It also built upon preliminary, previously reported analyses of the updated LTS (Turkheimer et al., 2015).

Comprehensive Wechsler IQ data (including Full Scale IQ, Verbal IQ, Performance IQ, and all subtest scores) were available for twins aged 7-15 years. Sample sizes ranged from 374 to 505 twin pairs (ages 15 and 8, respectively). We modeled the interaction of additive genetic (A) and shared environmental (C) variance in IQ with SES, which was also included as a linear and quadratic main effect (i.e., SES and SES2). We used a revised Purcell model of the interaction, which allows for phenotypic changes in variance that are independent of changes in the standardized twin correlations. The expected patterns of association (i.e., decreased heritability of IQ at low levels of SES and increased heritability at high levels) were observed for most analyses. However, the strength and significance of poverty $\times$ IQ heritability interaction fluctuated across ages and index scores/subtests in univariate analyses, likely due to a lack of power. Results of multivariate analyses (hierarchical and latent factor models) were more consistently significant.

\section{References}

Turkheimer, E., Haley, A., Waldron, M., D’Onofrio, B., \&; Gottesman, I.I. (2003). Socioeconomic status modifies heritability of IQ in young children. Psychological Science, 14, 623-628.

Turkheimer, E., Beam, C.R., Finkel, D., Davis, D.W., \&; Dickens, W.T. (2015). Investigating poverty by heritability interactions in the Louisville Twin Study. Paper presented at the Behavior Genetics Association annual meeting, San Diego, CA.

\section{A causal association between smoking and body mass index? Evidence from discordant twin pair design utilizing pooled data on 21 twin cohorts}

\section{Maarit Piirtola, University of Helsinki, Finland}

Background: Current daily smokers have generally been found to have lower body mass index (BMI) as compared to never or former smokers, but this association may have reversed in recent times. Because of genetic influences on both smoking and BMI, familial confounding (genetics and shared environment) is important to take into account to get more information about the causality of this association.

Aims: We investigated whether familial factors confound the associations between smoking status and BMI and whether these associations have changed over time.

Methods: Data on 55,290 same-sex twin pairs, 18-69 years of age, with 80,712 paired tests from 21 twin cohorts representing 14 countries were derived from the CODATwins database. We analyzed pairs discordant for their smoking status. One concurrent measurement of BMI and smoking status for each individual per a 10-year period (1960-1969, 1970-1979, 1980-1989, 1990-1999, and 2000-2010) was allowed. Conditional (fixed-effect) linear regression models adjusted for age and age-squared were used.

Results: Current daily smokers had lower BMI $(-0.37 \mathrm{~kg} / \mathrm{msup} 2 / \mathrm{sup}$, 95\% CI: $-0.43,-0.31$ in men, and $-0.44: 95 \%$ CI: $-0.52,-0.37$ in women) and former smokers higher BMI $(0.27,95 \%$ CI: $0.21,0.33$ in men, and 0.16 : $95 \% \mathrm{CI}: 0.08,0.23$ in women) compared to their never-smoker co-twins. Compared to current daily smokers, former smokers had higher BMI $(0.64,95 \%$ CI: $0.59,0.70$ in men, and 0.60 : $95 \%$ CI: $0.53,0.67$ in women). Similar trends were seen both in dizygotic and monozygotic pairs in both sexes across all 10-year periods except among former smokers. The BMI difference of former smokers compared to never-smokers attenuated in monozygotic pairs and was overall less clear in women.

Conclusions: Smoking is independently associated with lower BMI both in men and women, and this association has not changed over time. BMI change after smoking cessation may be confounded by genetics and other behavioral factors.

\section{Exploring the phenotypic structure and etiology of the big 5 personality dimensions in youth}

Ashley Watts, Emory University, United States; Holly Poore, Emory University, United States; Scott Lilienfeld, Emory University, United States; Irwin Waldman, Emory University, United States

Numerous researchers have examined the phenotypic structure of (McCrae et al., 1996) and genetic and environmental influences on 
(Loehlin, McCrae, Costa, \& John, 1998) adult personality, but little research has examined these issues in youth. The present study used a representative twin sample $(\mathrm{N}=507)$ aged $6-16(51 \%$ female $)$ to examine two specific aims. First, we tested alternative competing models for the phenotypic structure of youth personality using confirmatory factor analytic (CFA) and exploratory structural equation (ESEM) models. Second, we fit alternative biometric models to elucidate the etiology of the personality dimensions derived from the best-fitting phenotypic model. Consistent with our hypotheses, the factor analyses broadly supported the five-factor model in youth. Nevertheless, a strict five-factor CFA model did not fit as well as the five-factor ESEM model (Marsh et al., 2010). Full measurement and structural invariance across zygosity was met for the best-fitting ESEM model, suggesting that this factor structure replicated across MZ and DZ twins. All big five dimensions were substantially heritable, with estimates ranging from $42 \%$ (Neuroticism) to $77 \%$ (Openness to Experience). There also were moderate nonshared environmental influences, with estimates ranging from $58 \%$ (Neuroticism) to $23 \%$ (Openness to Experience), but no evidence for shared environmental influences. Significant rater contrasts were found for Extraversion and Openness to Experience, which is broadly consistent with rater contrasts detected in the ADHD literature (Freitag, Rohde, Lempp, \& Romanos, 2010), given the relevance of these traits (e.g., activity, cognitive flexibility) for ADHD (Martel et al., 2011). Taken together, our findings replicate and extend personality research on adults by suggesting that the big five domains can be reliably and validly assessed in youth, and that these dimensions are substantially heritable.

\section{Overlapping genetic influences on executive functions, general intelligence, and a general factor of psychopathology}

Paige Harden, University of Texas at Austin, United States; Laura Engelhardt, University of Texas at Austin, United States; Frank Mann, University of Texas at Austin, United States; Megan Patterson, University of Texas at Austin, United States; Andrew Grotzinger, University of Texas at Austin, United States; Stephanie Savicki, University of Texas at Austin, United States; Megan Thibodeaux, University of Texas at Austin, United States; Jennifer Tackett, Northwestern University, United States; Elliot Tucker-Drob, University of Texas, United States

Psychiatric disorders are widely comorbid, which can be represented as a latent factor, $p$, representing a general vulnerability to psychopathology. We used a population-based sample of child and adolescent twins $(n=1,913$, ages $7.8-20.1$ years, $M$ age $=13.1$ years, 49\% female, 58\% non-Hispanic White, 35\% monozygotic) from the Texas Twin Project (Harden KP, Tucker-Drob EM, Tackett JL). The Texas twin project. (Twin Res. Hum. Genet. 2013 Feb; 16(1):385) to examine the relationship between $p$ and cognitive abilities, specifically, executive functions (EFs) and general intelligence (g). Psychopathology was assessed using child self-report and parent report of 12 symptom domains, including thought problems, somatic problems, neuroticism, and symptoms of externalizing, internalizing, and attention-related DSM-defined disorders. Executive functions (EF) were measured using a battery of tests assessing inhibition, switching, updating, and working memory. Intelligence was measured using the WASI-2, comprising two tests of verbal reasoning and two tests of spatial reasoning. The mean full-scale IQ in the sample was $103(\mathrm{SD}=$ 14), mirroring population norms. Replicating previous work, a bifactor model with a general $\mathrm{p}$ factor fit well. Agreement between child- and parent-report of psychopathology was moderate $(r=.4$ for $p$ factor). Consistent with previous reports from this sample (Engelhardt LE, et al.
Genetic overlap between executive functions and intelligence. J. Exp. Psychol.-Gen. 2016 Sep;145(9):1141), latent factors representing general EF and $g$ were highly heritable $\left(\mathrm{h}^{2}=92 \%\right.$ for $\mathrm{EF}, \mathrm{h}^{2}=90 \%$ for $\left.\mathrm{g}\right)$ and were nearly synonymous genetically. Across the entire IQ range and across reporters, cognitive abilities were negatively related to $\mathrm{p}(\mathrm{r}$ $\sim-.25$ ) with a higher association at the low end of ability for parentreported psychopathology. The associations between cognitive abilities and $p$ were driven by shared genetic variants. Results are interpreted within a cognitive epidemiology framework.

\section{The association between personality disorders with alcohol use and misuse: a population-based twin study}

Elizabeth Long, Virginia Commonwealth University, United States; Steven Aggen, Virginia Commonwealth University, United States; Michael Neale Virginia Commonwealth University, United States; GunPeggy Knudsen, Norwegian Institute of Public Health, Norway; Robert Krueger, University of Minnesota, United States; Susan South, Purdue University, United States; Nikolai Czajkowski, University of Oslo, Norway; Ragnar Nesvag, Norwegian Institute of Public Health, Norway; Eivind Ystrom, Norwegian Institute of Public Health, Norway; Fartein Torvik, Norwegian Institute of Public Health, Norway; Kenneth Kendler, Virginia Commonwealth University, United States; Nathan Gillespie, Virginia Commonwealth University, United States; Ted Reichborn-Kjennerud, Norwegian Institute of Public Health, Norway

Background: Because previous studies have nearly always investigated single personality disorders (PDs), a clearer understanding of the etiological overlap between all DSM-IV PDs, alcohol use (AU), and alcohol use disorder (AUD) is needed (Bornovalova et al., 2013; Fu et al., 2002; McAdams et al., 2012). To our knowledge, no study has modeled the association between all 10 DSM-IV PDs and lifetime AU and AUD. The aim of the present study is to identify which PDs are mostly associated with the phenotypic, genetic, and environmental risks of lifetime AU and AUD, and to determine if these associations are stable across time.

Methods: Participants were Norwegian twins assessed at two waves. At Wave 1, 2,801 twins were assessed for all 10 DSM-IV PD criteria, lifetime AU, and DSM-IV AUD criteria. At Wave 2, six of the 10 PDs were again assessed along with AU and AUD among 2,393 twins. Univariate and multiple logistic regressions were run. Significant predictors were further analyzed using bivariate twin Cholesky decompositions. Results. Borderline and antisocial PD criteria were the best predictors of AU and AUD across the two waves. Despite moderate phenotypic and genetic correlations, genetic variation in these PD criteria explained only 4 and $3 \%$ of the risks in AU, and 5$10 \%$ of the risks in AUD criteria, respectively. At Wave 2, these estimates increased to 8 and 23\% for AU, and 17 and 33\% for AUD. Conclusions: Among a large Norwegian twin sample, borderline and antisocial PD criteria were the best predictors of the phenotypic and genotypic liability to AU and AUD. This effect remained consistent across time.

\section{Parental socioeconomic status moderates genetic influences on cognitive ability in a diverse sample of young adults}

Peter Barr, Virginia Commonwealth University, United States; Jeanne Savage, Virginia Commonwealth University, United States; Kenneth 
Kendler, Virginia Commonwealth University, United States; Danielle Dick, Virginia Commonwealth University, United States

Cognitive ability is an important predictor of multiple life outcomes including educational attainment and better health. And while cognitive ability is heritable, there are significant environmental influences that moderate genetic ability (Tucker-Drob and Bates; 2016; Psych Science 2, 138-149). We examined whether genomewide polygenic scores for educational attainment (EDU PGS) predict cognitive ability (SAT scores), and whether this influence is moderated by parental socioeconomic status (SES). Data for these analyses come from a longitudinal study of college students enrolled in a large, public university $(\mathrm{N}=5,953)$ who provided DNA. SAT scores were ascertained through student registry data. EDU PGS were created using summary statistics from a recent large scale GWAS of educational attainment (Okbay et al., 2016; Nature; 533, 539-542). Parental SES was derived from survey reports of parental education. We fit a series of linear models across ancestral population and meta-analyzed the results with sex and ancestry principal components as covariates. EDU PGS explained 1.5-3.5\% of the variance in SAT scores across ancestral groups. Main effects for EDU PGS and parental SES were significant in the meta-analysis. We found significant interactions between parental SES and EDU PGS for those of European and South Asian ancestry, such that the association between EDU PGS and SAT scores was er for those from high SES families. For those of African ancestry, parental SES moderated EDU PGS such that the association was er under conditions of lower SES. Genome-wide polygenic scores significantly predicted cognitive ability in a diverse sample of college students. Additionally, for some groups, genetic associations were er as parental SES increased, similar to previous findings among twins in US samples. These results underscore the importance of examining genetic and environmental interplay in complex outcomes and the heterogeneity of environmental influences of those from diverse backgrounds.

\section{Evidence for genetic correlation of DSM-IV opioid dependence and childhood trauma based on additive genome-wide effects}

Leslie Brick, Brown University, United States; Lauren Micalizzi, Brown University, United States; Marisa Marraccini, Brown University, United States; Valerie Knopik, Brown University, United States; Rohan Palmer, Emory University, United States

Purpose: The unprecedented increases in morbidity and mortality associated with the opioid epidemic are indicative of the worst drug epidemic in US history. Previous research has demonstrated a moderate genetic correlation $\left(\mathrm{r}_{\mathrm{G}}=.49\right)$ between interpersonal trauma and generalized vulnerability to drug dependence (Palmer et al., 2016). The current study aims to expand on this work and investigate whether common genetic markers implicated in childhood trauma are implicated in opioid dependence (OD).

Methods:Genome-wide data ( $\mathrm{N}=3,894$ unrelated individuals) were drawn from several studies and imputed, including the Comorbidity and Trauma Study, Heroin Dependence in Western Australia, OZALC, and ongoing genetic studies of substance dependence at Yale. Case/control genomic-relatedness-matrix restricted maximum likelihood estimation was used to determine the proportion of variance in each phenotype attributed to additive genetic variance $\left(h^{2}{ }_{S N P}\right)$. Bivariate models were used to determine the genetic correlation $\left(\mathrm{r}_{\mathrm{G}}\right.$ -SNP) across the phenotypes.

Analyses controlled for sex, age, and other drug dependence and parameter estimates for OD were adjusted using a transformation to a liability scale based on disease prevalence in the population. A subset of individuals $(\mathrm{N}=1,346)$ had data on childhood trauma.

Results: Moderate additive genetic influences on ODem, $\mathrm{h}^{2} \mathrm{SNP}-\mathrm{OD}$ $=.41(\mathrm{SE}=.03, \mathrm{p}<.001)$ are partially shared with additive genetic effects on childhood trauma, $\mathrm{h}_{\mathrm{SNP}-\mathrm{Trauma}}^{2}=.44(\mathrm{SE}=.24, \mathrm{p}=.03) ; \mathrm{r}_{\mathrm{G}}$ $-\mathrm{SNP}-\mathrm{OD}-\mathrm{Trauma}=.48(\mathrm{SE}=.06, \mathrm{p}<.001)$. The heritability of OD for males and females was similar $\left(\mathrm{h}^{2}\right.$ SNP-Males $=.47, \mathrm{SE}=.06, \mathrm{p}<.001$; $\left.\mathrm{h}^{2}{ }_{\text {SNP-Females }}=.33, \mathrm{SE}=.08, \mathrm{p}<.001\right)$ and highly correlated, $\mathrm{r}_{\mathrm{G}-\mathrm{NP}}$ -Males-Females $=.87(\mathrm{SE}=.16, \mathrm{p}<.001)$.

Conclusion: Findings suggest that a moderate portion of the additive genetic effects on trauma is shared with the additive genetic effects on OD. Further, the high $\mathrm{r}_{\mathrm{G}-\mathrm{SNP}}$ across males and females for OD indicates that largely the same polymorphisms contribute to heritability across each sex, despite findings from previous literature suggesting that genetic influences across males and females may be differential. Potential reasons for this overlap will be discussed along with trends of h2SNP stratification across the genome.

\section{Heritability of spontaneous eye blink rate}

Andrey Anokhin, Washington University in St. Louis, United States; Simon Golosheykin, Washington University, School of Medicine, United States

Spontaneous eye blink rate (EBR) is a simple involuntary behavior that can be measured unobtrusively in a variety of settings. EBR shows substantial inter-individual variability and has been associated with differences in cognitive function, personality, and psychopathology. Increasing evidence suggests that EBR can serve as an indirect indicator of central dopamine function. However, little is known about the extent to what individual differences in EBR are influenced by genetic factors. Here we investigated heritability of EBR in a population-based sample of young adult twins. Eye blinks were detected using an electro-oculographic (EOG) recording in a resting-state condition while the subjects were instructed to keep their gaze on a fixation cross. Four 1-min resting periods were sampled during a 2-h laboratory session, and individual EBR was computed as the average number of blinks per minute. Linear structural equation modeling of twin data showed that $55 \%$ of EBR variance can be attributed to additive genetic factors, with little evidence for shared environmental influences.

\section{Association between the oxytocin receptor gene (OXTR) and childhood aggression}

Holly Poore, Emory University, United States; Devon LoParo, Emory University, United States; Rohan Palmer, Emory University, United States; Hasse Walum, Emory University, United States; Kimberly Kerley, Emory University, United States; Larry Young, Emory University, United States; Kerry Ressler, Harvard University, United States; Irwin Waldman, Emory University, United States

Human aggression has important implications for long-term life outcomes and is a complex trait, influenced by a multitude of factors. Behavior genetic studies have found that aggression is highly heritable across the lifespan. Despite its high heritability, molecular genetic studies of aggression have had mixed results, with few genetic variants showing reliable associations. Recent research suggests that Oxytocin and the Oxytocin Receptor Gene (OXTR) influences social cognition and behavior in humans and animal models. The primary aim of this study is to examine associations between OXTR and measures of aggression in children and adolescents. Based on current 
animal models, it is hypothesized that OXTR will be associated with various forms of aggression. In the current investigation we collected DNA and parent ratings of aggression for a total of 636 children ages 6-18 years old sampled from both unreferred twins as well as clinically-referred children. The investigation included 31 SNPs in OXTR. To operationalize OXTR in a gene-based test, a series of Exploratory Structural Equation Models (ESEMs) of the OXTR /SNPs were conducted. The model with five factors fit best while still favoring parsimony and was used to characterize the underlying structure of the gene. Aggression was also modeled as both a unitary aggression factor and as two separate reactive and proactive aggression factors. A series of gene-based tests were then conducted such that the aggression factors were regressed on the latent OXTR factors and all covariates. The OXTR factors accounted for a significant portion of the variance in the unitary aggression factor $\left(\mathrm{R}^{2}=1.8 \%, \mathrm{p}<.001\right)$, reactive aggression $\left(\mathrm{R}^{2}=1.2 \%, \mathrm{p}<.001\right)$, and proactive aggression $\left(\mathrm{R}^{2}=5.8 \%, \mathrm{p}<.001\right)$ over and above the contribution of the covariates. Future research will focus on replicating these results in larger samples from a diverse group of cohorts.

\section{Genetic analysis reveals etiologic heterogeneity in major depression}

Roseann Peterson, Virginia Institute for Psychiatric and Behavioral Genetics, United States; Na Cai, Wellcome Trust Sanger Institute, United Kingdom; Tim Bigdeli, SUNY Downstate, United States; Alexis Edwards, Virginia Institute for Psychiatric and Behavioral Genetics, United States; Bradley Webb, Virginia Institute for Psychiatric and Behavioral Genetics, United States; Jonathan Flint, UCLA, United States; Kenneth Kendler, Virginia Commonwealth University, United States

Results from genome-wide association studies have led to the view that the genetic architecture of psychiatric disorders arises primarily from multiple variants each contributing a small effect to disease risk. Using a highly selected cohort of 4,785 women with recurrent major depression (MD) and 4,814 screened controls, we show that the $26 \%$ of cases who report major adverse life events only partly share genetic risk factors with the $74 \%$ who did not. We report three loci whose effects are only significantly associated with MD in those reporting no history of adversity. Significant interaction occurs between self-reported adversity and genotype at all three loci. Findings are consistent with etiologic heterogeneity within MD. Our results indicate that the relationship between environmental and genetic susceptibility varies across loci, and have implications for the molecular dissection of MD and other complex traits.

\section{Increased heritability of disordered eating across puberty: a replication in a developmentally diverse sample}

Laura Mayhall, Michigan State University, United States; Shannon O'Connor, Michigan State University, United States; Kristen Culbert, University of Nevada, Las Vegas, United States; S. Alexandra Burt, Michigan State University, United States; Kelly Klump, Michigan State University, United States

Objective: Previous twin research has shown that genetic influences on disordered eating symptoms increase across puberty (Culbert, Burt, McGue, Iacono, \& Klump, J Abnorm Psychol, 2009; Klump, McGue, \& Iacono, Int J Eat Disord, 2003; Klump, Perkins, Burt, McGue, \& Iacono, Psychol Med, 2007). However, almost all studies were conducted in the same sample of twins, and this sample had limited variability in pubertal development (e.g., studying twins who were age 11 or age 14 only). The current study aimed to replicate past findings in a new twin sample that included the full range of variability in age (ages 8-25 years old) and pubertal development (prepuberty through post-puberty).

Methods: Participants included 1,568 same-sex female twins from the Michigan State University Twin Registry. Disordered eating symptoms were assessed with the total score (assessing body dissatisfaction, weight preoccupation, binge eating, and compensatory behaviors) from the Minnesota Eating Behavior Survey, while pubertal development was assessed with the Pubertal Development Scale.

Results: Twin moderation models showed significant linear increases in genetic effects from pre-puberty (3\%) to post-puberty (45\%), even after controlling for the effects of age and body mass index (BMI). Discussion: Results replicated previous findings that genetic effects on disordered eating increase across pubertal development. Because analyses controlled for other relevant factors (i.e., age and BMI), these results indirectly suggest that ovarian hormones, a key element in pubertal development, may be responsible for this increase in heritability. Further research should directly determine the role of ovarian hormones in this heritability increase.

\section{Genome-wide analyses of smoking behaviors in schizophrenia: findings from the Psychiatric Genomics Consortium}

Roseann Peterson, Virginia Institute for Psychiatric and Behavioral Genetics, United States; Tim Bigdeli, SUNY Downstate, United States; Schizophrenia Working Group Of The Psychiatric Genomics Consortium, United Kingdom; Kenneth Kendler, Virginia Commonwealth University, United States; Ayman Fanous, SUNY Downstate, United States

Objective: While $17 \%$ of US adults use tobacco regularly, smoking rates among persons with schizophrenia (SCZ) spectrum disorders are upwards of $60 \%$. Several lines of evidence support a shared etiological basis for smoking and SCZ, including recent findings from genome-wide association studies (GWAS). However, few studies have considered whether genetic variants also influence smoking behavior among SCZ cases.

Methods: We combined single nucleotide polymorphism (SNP) genotypes with self-reported nicotine-use and symptom data from the Psychiatric Genomics Consortium (PGC) study of SCZ. We evaluated whether polygenic risk scores (PRS) constructed from the results of the Tobacco and Genetics (TAG) meta-analyses of smoking behaviors are associated with SCZ risk, or predict these same outcomes in cases. Using genome-wide summary statistics for SCZ, and results from exploratory case-only GWAS of smoking initiation (SI) and cigarettes-smoked-per-day (CPD), we estimated the genetic correlation with TAG phenotypes.

Results: We demonstrate significant genetic correlations of SCZ with SI $(\rho g=0.159 ; \mathrm{P}=5.05 \times 10-10), \mathrm{CPD}(\rho g=0.094 ; \mathrm{P}=.006)$, and ageof-onset of smoking ( $\rho g=0.1 ; \mathrm{P}=.009)$ in the general population, and successfully replicate findings for SI and age-of-onset in an independent East-Asian cohort. Comparing SCZ-cases to the general population, we observe a significant positive genetic correlation for SI $(\rho g=0.624, P=.002)$. Similarly, TAG-based PRS for SI and CPD were significantly associated with SI $\left(\mathrm{P}=3.49 \times 10^{-5}\right)$ and $\mathrm{CPD}(\mathrm{P}=.007)$ among cases. We also successfully replicated a novel SNP association with CPD among cases upstream of TMEM106B on chromosome 7 (rs148253479; $\left.\mathrm{P}=3.18 \times 10^{-8}, \mathrm{~N}=3,520\right)$. 
Conclusion: We provide evidence of a partially shared genetic basis for SCZ and smoking behaviors, and for smoking behaviors among SCZ patients and the general population. Future research needs to address mechanisms underlying associations between these traits to aid both SCZ and smoking treatment and prevention efforts.

\section{Genetic and environmental contributions to level and change in conscientiousness among Japanese adolescent twins}

Yusuke Takahashi, Kyoto University, Japan; Koken Ozaki, University of Tsukuba, Japan; Shinji Yamagata, Kyushu University, Japan; Juko Ando, Keio University, Japan

In this study, using genetically informed longitudinal analysis, we sought to examine the genetic and environmental contributions to stability and change in conscientiousness, one of the big five personality traits. Although the stability was due to genetic influence according to accumulated evidence on heritability of various variables, the etiology of changes is more likely to be unclear. We then focused more on changes in conscientiousness to test whether the variance of changes was significant, and to what extent the variance of changes can be explained by the genetic or environmental factor. We used two-wave longitudinal data collected from Japanese adolescent twins whose ages ranged from 9 to 18. Data collections were 1 year apart. Completed online questionnaires were received from 2,022 twin pairs, comprising $708 \mathrm{mMZ}, 671 \mathrm{fMZ}, 111 \mathrm{mDZ}, 110$ fDZ, and $422 \mathrm{oDZ}$ pairs at Wave 1, and from 1,285 twin pairs, comprising $436 \mathrm{mMZ}, 445 \mathrm{fMZ}, 71 \mathrm{mDZ}, 62 \mathrm{fDZ}$, and $271 \mathrm{oDZ}$ pairs at Wave 2. Conscientiousness was assessed by the following five scales: Conscientiousness items form Big Five Inventory-44, Chernyshenko conscientiousness scales, Effortful Control items from Adult Temperament Questionnaire, Self-control scale, and grit scale. At both time points, common pathway models fit best to the data, and there was a heritable general factor $\left(h^{2}=72.1,70.6 \%\right.$, respectively). Latent change analysis with longitudinal twin data revealed that cross-time stability was largely accounted for by genetic influence $(66.1 \%)$, whereas change over time was by non-shared environmental influence $(63.0 \%)$. Our results demonstrated that heritability for level was greater than heritability for change, while environmentability for change was greater than environmentability for level, suggesting that the stability of conscientiousness over time appears to be primarily a function of enduring genetic influences, and that non-shared environmental influence is primarily responsible for change in conscientiousness over time. In addition, there was a high non-shared environmental correlation between level and change $(r=-.623)$, indicating that the non-shared environmental factor that affects the stability of trait conscientiousness influences change in opposite direction.

\section{Substance abuse and criminality in the offspring of substance abusing parents: children-of-siblings analyses in nationwide register data}

Antti Latvala, University of Helsinki, Finland; Ralf Kuja-Halkola, Karolinska Institutet, Sweden; Nitya Jayaram-Lindström, Karolinska Institutet, Sweden; Henrik Larsson, Karolinska Institutet, Sweden; Paul Lichtenstein, Karolinska Institutet, Sweden

Parental substance abuse (SA) is associated with elevated risks for substance use problems and antisocial behavior in the offspring.
The children-of-twins design has been used to distinguish direct parental effects from genetic confounding but studies have been based on just two different datasets of twin parents and relatively small samples. Using data from Swedish nationwide registries, we conducted children-of-siblings analyses in offspring of monozygotic and dizygotic twin, full sibling, and half-sibling parents. Parental SA was identified from medical and legal registries as diagnoses of alcohol/drug use disorders and alcohol/drug related criminal convictions. Offspring SA and any criminal convictions were identified from the same registries. Individual-level analyses were conducted among nearly 2.5 million offspring, born 1958-1995, of whom $15 \%$ had paternal and 5\% had maternal SA registrations. Both maternal and paternal SA more than doubled the risk for offspring SA (maternal hazard ratio $[\mathrm{HR}]=2.81$ [95\% CI: 2.77-2.86]; paternal $\mathrm{HR}=2.51$ [2.49-2.54]) and criminality (maternal $\mathrm{HR}=2.20$ [2.17-2.23]; paternal $\mathrm{HR}=2.07$ [2.05-2.09]). Associations were attenuated in stratified analyses among full cousins suggesting genetic confounding. Using data from cousins and siblings from the different extended families $(n=1.6$ million) we conducted quantitative genetic structural equation modeling to estimate the proportions of variance and covariance for parental SA and the offspring outcomes. Additive genetic effects were estimated to explain $42 \%$ (95\% CI: $28-56 \%$ ) and $46 \%$ (36-55\%) of the variance in maternal and paternal SA, respectively, and between $37 \%$ $(31-44 \%)$ and $53 \%(47-58 \%)$ of the variance in the offspring outcomes. Only additive genetic effects significantly contributed to the intergenerational associations between paternal SA and the offspring outcomes as well as maternal SA and offspring criminality. In contrast, the association between maternal and offspring SA was due to genetic (54\% [95\% CI: 25-84\%]), extended family environmental $(17 \%[2-31 \%])$ and nuclear family environmental (29\% [12-45\%]) components, suggesting also potential environmental transmission.

\section{Childhood aggression: results from the ACTION and EAGLE Consortia}

Tellervo Korhonen, University of Helsinki, Finland; Meike Bartels, Vrije Universiteit Amsterdam, Netherlands; Dorret Boomsma, Vrije Universiteit Amsterdam, Netherlands

Tellervo Korhonen, Meike Bartels and Dorret Boomsma on behalf of the ACTION consortium.

Childhood aggression and its comorbidities result in a huge burden on affected children, their relatives, teachers, peers, and society as a whole. This symposium is based on data analysed within the ACTION (Aggression in Children: Unravelling geneenvironment interplay to inform Treatment and InterventiON strategies) and EAGLE (EArly Genetics and Lifecourse Epidemiology) consortia and focuses on phenotypes and (genetic) epidemiology analyses, including achievements in phenotype harmonisation of aggressive behaviour, comorbidities from teacherrated behavioural and emotional problem data, and comorbidity between aggression and academic performance. Based on epidemiological data from Sweden, we present the first results regarding childhood aggression and adverse outcomes in young adulthood. This set of presentations is followed by presentations of results from the first large GWA (Genome-wide Association) on aggression in children and adults and a meta-analysis of EWA (Epigenome-wide Association) studies across the lifespan. We carried out a multivariate, multi-rater and multi-age GWAS of aggression and attention problems and present the genetic correlations with adult psychiatric outcomes. 


\section{Early life stress and genetic risk for neuroticism predicting health outcomes in older Swedish twins}

Kelli Lehto, Karolinska Institutet, Sweden; Ida Karlsson, Karolinska Institutet, Sweden; Cecilia Lundholm, Karolinska Institutet, Sweden; Nancy L. Pedersen, Karolinska Institutet, Sweden

Introduction: High neuroticism is a predictor of many negative health outcomes and lower well-being in older populations. However, neuroticism itself is likely shaped by gene-environment (GE) interplay already early in life and thus, these genetic and childhood environmental factors might influence various health outcomes across the life span. To date, the interplay between early life stress and genes underlying neuroticism remain poorly understood. Since neuroticism has a complex genetic architecture, a polygenic risk score approach would help to further improve our understanding of such GE interplay.

Aim: This study explored how early life stress exposure, polygenic risk scores for neuroticism (PRSn) and their interplay influence psychosocial and health outcomes in the second half of the life-span.

Methods: The Swedish Adoption/Twin Study of Aging followed twins reared apart and matched twins reared together over a 30-year time period. The current study uses cross-sectional data from the first measurement occasion, resulting in a total of 1956 individuals. Genotype data were available for a subset of 637 individuals. Rearing status (reared apart or together) is considered an objective indicator of early life stress (median age at separation $=2$ ). PRSn were created at seven $\mathrm{p}$-value thresholds. Outcomes included psychosocial factors (socioeconomic status, life stress, family environment) as well as several emotional health (neuroticism, depressive and anxiety symptoms, life satisfaction) and physical health (self-rated health, disease burden, functionality level) measures.

Results: Early stress exposure predicted higher levels of neuroticism, life stress and loneliness, and lower life satisfaction and self-rated health in late-life. PRSn consisting of approximately 10000 SNPs under p-value threshold of .01 was the most powerful predictor of measured neuroticism late in life. PRSn also predicted high depressive symptoms, loneliness and poor family environment. Interestingly, rearing status and PRSn interacted in predicting depressive symptoms and loneliness, wherein genetic effects were higher in twins not exposed to early life stress, indicating possible underlying gene-environment interplay.

Conclusions: These results suggest the importance of early life stress and genetic predisposition to emotional instability in shaping health and well-being in old age.

\section{Symposium: risk tolerance}

Philipp Koellinger, Vrije University, Netherlands; Renato Frey, University of Basel, Switzerland; Paige Harden, University of Texas at Austin, United States; Richard Karlsson Linnér, Vrije Universiteit Amsterdam, Netherlands; Edward Kong, Harvard University, United States

Risk tolerance, or the willingness to take risks to obtain rewards, is a fundamental parameter in a wide range of theories in economics, finance, and behavioral decision theory. Different measures of risk preferences have previously been linked to real-world behaviors such as portfolio allocation, occupational choice, as well as health behaviors such as smoking, exercise, alcohol and drug use. General risk tolerance has been found to be moderately heritable in twin studies-with heritability estimates ranging from 20 to $60 \%$. Yet, little is known about the molecular genetic architecture of risk tolerance and related risky behaviors. Our symposium presents novel evidence on four related questions:

(1) How are different measures of risk tolerance related on a phenotypic level? Is there a general factor of risk preference, $\mathrm{r}$ akin to g, the general factor of intelligence-that complements domain- and situation-specific dimensions?

(2) Do different phenotypic measures of risk taking share a common genetic architecture?

(3) Is it possible to identify specific, replicable genetic associations for risk tolerance and risky behaviors with currently available GWAS sample sizes?

(4) How are risk tolerance and risky behaviors linked to mental and physical health?

\section{Drinking motives underlying internalizing and externalizing pathways to alcohol misuse in college students}

Jeanne Savage, Virginia Commonwealth University, United States; John Hettema, Virginia Commonwealth University, United States; Roxanne Roberson-Nay, Virginia Commonwealth University, United States; Alexis Edwards, Virginia Commonwealth University, United States; Nathan Gillespie, Virginia Commonwealth University, United States; Kenneth Kendler, Virginia Commonwealth University, United States; Danielle Dick, Virginia Commonwealth University, United States

Alcohol misuse is widespread among college students and is believed to have a heterogeneous genetic and environmental etiology. Different types of drinking motives, such as drinking to cope with stress versus drinking to enhance positive feelings, provide a theoretical mechanism behind distinct "internalizing" and "externalizing" pathways to alcohol misuse. Drinking motives are heritable and are robust proximal predictors of alcohol misuse; however, little is known about the nature of these relationships, and whether they are due to a shared liability or direct etiological mechanisms. The nature of the relationships between drinking motives and internalizing/externalizing psychopathology is also uncertain. Our aim is therefore to test different models of the relationships between these outcomes using a genetically informative longitudinal sample of college students ("Spit for Science", $\mathrm{N}=$ 9,889 ) who completed self-report surveys with measures of drinking motives, alcohol use disorder symptoms (AUDsx), and internalizing and externalizing (INT/EXT) psychopathology. Cross-lagged panel analyses were used to test direct phenotypic relationships between these outcomes across the college years. Genome-wide complex trait analysis (GCTA) and LD score regression (LDsc) were used to test for a shared genetic etiology. Cross-lagged model results indicated a higher directional association from drinking motives to AUDsx than vice versa, indicative of a direct mechanism by which drinking motives shape alcohol misuse. Shared liability, or reciprocal causation, appeared to drive the associations between INT/EXT and drinking motives. Preliminary results from GCTA/LDsc suggest genetic overlap across types of motives, but not between motives and INT. The findings help to clarify the nature of the relationships between drinking motives, alcohol misuse, and internalizing and externalizing psychopathology, and indicate a mechanism by which drinking motives directly influence alcohol misuse. Future analyses will further investigate shared genetic etiologies to provide insight into the role that drinking motives play in internalizing and externalizing pathways to alcohol misuse. 
A modified Purcell model for the analysis of heritability by socioeconomic status interactions

Eric Turkheimer, University of Virginia, United States; Christopher Beam, University of Southern California, United States; Evan Giangrande, University of Virginia, United States; Sarah Carroll, University of Virginia, United States

We propose a structural equation model, which we call a modified twin correlation model, for the analysis of pair-level modifiers of biometric analyses continuous phenotypes. The standard model for this type of analysis, which was developed by Purcell (2002), expresses biometric modification of ACE variances by modeling paths between standardized ACE variances and a phenotype as quadratic functions of the moderator. Our proposed modifications of this highly successful model are in response to several theoretical and empirical considerations. First, we have commonly observed that phenotypic variance is systematically related to the moderator: in particular, phenotypic variance of cognitive ability is often reduced in high quality environments. Reductions in phenotypic variance are confounded with changes in ACE components; our model treats changes in phenotypic variance and changes in standardized components separately. Second, we have recently observed that modification models of ACE components of cognitive ability sometimes lead to estimates outside the permitted parameter space of the classical twin model, especially by predicting DZ twin correlations that are less than half the corresponding MZ correlation. Our modified model therefore focuses the analysis on changes in the twin correlations themselves, rather than on the ACE components that are derived from them. Finally, when modeling variances we prefer exponential to quadratic models. Exponential models have the advantage of being monotonic with respect to the modifier, and they are more consistent with similar models for heteroscedasticity in the general analysis of variance literature. Parameters describing the ACE components can be added to the model if they are desired. We demonstrate the validity of the model by analyzing simulated data with known parameters. We then apply it to cognitive ability data from Norwegian conscripts, longitudinal data from the Louisville Twin Study, and the contemporary TwinLife project in Germany. Results are compared to those obtained using the classical twin model.

\section{References}

Purcell, S. (2002). Variance components models for gene-environment interaction in twin analysis. Twin research, 5/(06), 554-571.

\section{Childhood psychosocial home environment modifies genetic and environmental influences on leisure-time physical activity in young adulthood}

Sari Aaltonen, University of Helsinki, Finland; Richard Rose, Indiana University, United States; Urho Kujala, University of Jyvaskyla, Finland; Lea Pulkkinen, University of Jyvaskyla, Finland; Jaakko Kaprio, University of Helsinki, Finland; Karri Silventoinen, University of Helsinki, Finland

Both genetic factors and childhood home environment explain individual differences in physical activity, but the interaction between them is poorly understood. This study aims to examine to what extent childhood psychosocial home environment modifies the relative roles of genetic and environmental influences of leisure-time physical activity in young adulthood. The participants were Finnish twins $(\mathrm{N}=$ 3305) who self-reported their leisure-time physical activity at age 24. Psychosocial childhood home environment was assessed by twins at ages 12,14 and 17 as well as by parents when the twins were 12 years old. Statistical modeling of gene-environment interaction was carried out with OpenMx software. Twins' ratings of higher positive home atmosphere at ages 14 and 17 along with lower relational tensions at home at ages 12 and 14 predicted higher leisure-time physical activity levels in young adulthood (regression coefficients from 0.35 (95\% CI $0.02-0.67)$ to 0.84 (95\% CI $0.47-1.20)$ ). Twins' perceived positive home atmosphere at age 14 significantly increased both the additive genetic variation (moderation effect 0.59 (95\% CI $0.15-0.99)$ ) and specific environmental variation (moderation effect 0.55 (95\% CI 0.31-0.74)) of leisure-time physical activity in young adulthood. The lower levels of relational tensions rated by twins at ages 12 and 14 increased also the specific environmental variation in leisure-time physical activity (moderation effects 0.54 (95\% CI $0.26-0.69)$ and 1.41 (95\% CI 0.69-1.95), respectively). Similarly, parents' ratings of lower levels of relational tensions were associated with the increase of specific environmental variation (moderation effect 0.44 (95\% CI 0.02-0.62)) in twins' physical activity, while the additive genetic variation was significantly decreased (moderation effect -0.41 (95\% CI -0.80 to -0.02$)$ ). Our study suggests that warm and emotionally supportive childhood home environment can increase the level as well as genetic and environmental variability of leisure-time physical activity several years later.

\section{Shared genetic variants in speech and language traits}

Chin Yang Shapland, Max Planck Institute of Psycholingiustics, Netherlands; Ellen Verhoef, Max Planck Institute of Psycholinguistics, Netherlands; George Davey Smith, University of Bristol, United Kingdom; Susan M. Ring, University of Bristol, United Kingdom; Simon E. Fisher, Max Planck Institute for Psycholinguistics, Netherlands; Beate St Pourcain, Max Planck Institute for Psycholinguistics, Netherlands

Introduction: Oral and written language abilities during childhood are related to educational attainment, occupational success and health during later life. Many of these traits are highly heritable and share genetic links with verbal intelligence quotient (VIQ) scores. The nature of this shared genetic basis is, however, not well understood. We aim to identify and characterise the proportion of genetic variation in the genome, as tagged by common markers, that can explain phenotypic variance across multiple oral and written language abilities during childhood.

Method: We studied 13 traits (comprehension, reasoning, reading, spelling, phonological awareness/memory abilities) in 7-12 years-old children from the Avon Longitudinal Study of Parents and Children $(\mathrm{N} \leq 5,919)$. The traits were heritable $\left(\mathrm{SNP}-\mathrm{h}^{2}\right.$ from $0.25(\mathrm{SE}=0.07)$ to $0.49(\mathrm{SE}=0.07))$ and genetically correlated with VIQ scores $\left(\mathrm{r}_{\mathrm{g}}\right.$ from $0.62(\mathrm{SE}=0.10)$ to $0.89(\mathrm{SE}=0.07))$. We carried out a cross-phenotype meta-analysis of correlated traits based on GWAS summary statistics to identify genetic variants that share similar effects across traits (core-SNPs), restricting the analysis to HapMap3 SNPs.

Results: Tagging $\sim 10 \%$ of the genome, the core-SNPs explained a large proportion of SNP- $\mathrm{h}^{2}$ per trait (minimum enrichment 11-fold ( $\mathrm{SE}=0.22), \mathrm{P}=1 \times 10^{-5}$ ), although these results might be inflated because of over-fitting. Evidence for enrichment was also observed for performance IQ scores (10-fold ( $\mathrm{SE}=0.21), \mathrm{P}=8 \times 10^{-6}$ ), but not for height $(\mathrm{P}=.06)$ or gestational age $(\mathrm{P}=.32)$. An analysis of coreSNPs in the UK Biobank ( $N=36,035,40-69$ years) also showed some evidence of enrichment with respect to fluid IQ scores (twofold ( $\mathrm{SE}=$ $0.03), \mathrm{P}=.004$ ) and its verbal subset (twofold $(\mathrm{SE}=0.03), \mathrm{P}=.02$ ).

Discussion: Our analyses suggest that a defined set of variants in the genome can explain a large proportion of phenotypic variance across multiple traits that require directly and indirectly the mastering of 
language skills. However, genetic variants contributing to phenotypic variation during childhood may differ from those in adulthood, and our findings require further replication in age-matched populationbased cohorts

\section{Estimating developmental changes within the genetic architecture of social communication traits: a multivariate study of genetic variance in unrelated individuals}

Beate St Pourcain, Max Planck Institute for Psycholinguistics, Netherlands; Lindon Eaves, Virginia Commonwealth University, United States; Ellen Verhoef, Max Planck Institute of Psycholinguistics, Netherlands; Chin Yang Shapland, Max Planck Institute of Psycholingiustics, Netherlands; Susan M. Ring, University of Bristol, United Kingdom; Simon E. Fisher, Max Planck Institute for Psycholinguistics, Netherlands; Sarah Medland, QIMR, Brisbane, Australia; David Evans, University of Queensland, Australia; George Davey Smith, University of Bristol, United Kingdom

Background: Social-communication problems are heritable traits that share genetic influences with multiple psychiatric conditions, as tagged by common variants. The degree of trait-disorder overlap depends, however, on the developmental stage of the behaviour studied. This suggests changes in the genetic trait architecture that are part of human maturation processes. We developed a multivariate analysis framework in unrelated individuals to assess the developmental profile of genetic influences contributing to socialcommunication difficulties during a $\sim 10$-year period spanning childhood and adolescence.

Methods: Standardised longitudinally assessed quantitative socialcommunication problems ( $\mathrm{N} \leq 5,551$, Social Communication Disorders Checklist, 8-17 years) were studied in children and adolescents from the Avon Longitudinal Study of Parents and Children (ALSPAC), a UK birth cohort. Genetic influences across development were investigated using multivariate genetic-relationship-matrix structural equation models (GSEM). This analysis combines whole-genome genotyping information with structural equation modelling techniques. Analogous to twin research, GSEM included a Cholesky decomposition model, a common pathway model and an independent pathway model.

Results: A Cholesky decomposition model, specifying two distinct genetic factors arising during childhood, fitted the data best. One genetic factor was common to SCDC measures across development (path coefficients: $0.20(\mathrm{SE}=0.07)$ to $0.54(\mathrm{SE}=0.07))$. The other factor accounted for independent phenotypic variation at 11 and 17 years of age (path coefficients: $0.20-0.44)$. Genetic factors operating at age 8 years explained $\sim 60 \%(\mathrm{SE}=0.13)$ of genetic variation at age 17 years.

Conclusion: We identified dynamic changes in genetic factors influencing social-communication difficulties during child and adolescent development, consistent with distinct developmental trait profiles in genetic overlap with psychiatric illness. Our findings have relevance for studies investigating shared genetic aetiologies across multiple phenotypes.

\section{Quadratic effects of socioeconomic status on IQ}

Lucas Matthews, University of Virginia, United States; Evan Giangrande, University of Virginia, United States; Sarah Carroll, University of Virginia, United States; Eric Turkheimer, University of Virginia, United States
Previous behavior genetic research has demonstrated correlations between socioeconomic status (SES) and IQ; higher SES is associated with higher IQ while lower SES is associated with lower IQ. While these associations implicate a significant environmental component to intelligence, the heritability of IQ is also well documented. Further research demonstrates, however, that SES moderates IQ heritability in American populations; the heritability of intelligence is enhanced by higher SES and diminished by lower SES. Analyses by Tucker-Drob (2013) and Ritchie (2015) have identified developmental pathways underlying SES differences in the development of cognition and achievement. Here we advance this research by conducting similar longitudinal analyses using a genetically informative, longitudinal design. While previous studies evince a linear effect of SES on IQ, our analysis reveals a consistently nonlinear quadratic effect, with a negative relationship in which the slopes of IQ are greater at lower levels of SES. The effect is greatest for PIQ and Fluid IQ.

Analyzing data on 365 monozygotic and 376 dizygotic twin pairs from the Louisville Twin Study, we assess the quadratic effect of SES by IQ. The Louisville twins were administered full WISC tests up to five times between the ages of seven and 15 . We examine the extent to which the effects of SES on subtest performance are mediated by $\mathrm{g}$. We then decompose the relationship between SES and subtests into ACE components, and use these components to explore SES quadratic effects on stability and change in $\mathrm{g}$ and subtest performance over time. Finally, we develop two factor models of WISC subtests in which the rotation of the factors is determined by differential relations with SES rather than simple structure.

\section{References}

Ritchie, Stuart J., Timothy C. Bates, and Ian J. Deary. 2015. "Is Education Associated with Improvements in General Cognitive Ability, or in Specific Skills?" Developmental Psychology 51 (5): 573-82.

Tucker-Drob, Elliot M. 2013. "How Many Pathways Underlie Socioeconomic Differences in the Development of Cognition and Achievement?" Learning and Individual Differences 25 (June): 1220.

\section{Ecstasy use: demographics, heritability and genetic association with use of other substances}

Karin Verweij, Behavioural Science Institute, Australia; Jorien Treur, Behavioural Science Institute, Netherlands; Annabel Vreeker, Trimbos Instituut, Netherlands; Abdel Abdellaoui, Vrije Universiteit Amsterdam, Netherlands; Jouke Hottenga, Vrije Universiteit Amsterdam, Netherlands; Gonneke Willemsen, Vrije Universiteit Amsterdam, Netherlands; Tibor Brunt, Trimbos Instituut, Netherlands; Dorret Boomsma, Vrije Universiteit Amsterdam, Netherlands; Jacqueline Vink, Radboud University, Behavioural Science Institute, Netherlands

Ecstasy is a widely used psychoactive drug that users often take because they experience positive effects such as increased euphoria, sociability, elevated mood, and heightened sensations. Ecstasy use is not harmless and several immediate and long term side effects have been identified. We examined the demographics of ecstasy users and determined the relationship of ecstasy use with use of other substances (legal and illegal). We also estimated the heritability of ecstasy use and the genetic association of ecstasy use with smoking, alcohol, and cannabis use. The sample comprised of 8,500 twins and siblings aged 18-45 years from twin families registered at the Netherlands Twin Registry. In 2013-2014 participants filled out a questionnaire including questions about ecstasy use. A subset of these 
individuals ( $\mathrm{N} 4000$ ) were also genome-widely genotyped. We used the classical twin design to partition the individual differences in liability to ecstasy use into genetic, shared environmental, and residual components. Moreover, we created polygenic risk scores for tobacco, alcohol, and cannabis use and determined to what extent these polygenic scores could predict ecstasy use. Approximately $10 \%$ of the sample had used ecstasy during their lifetime, with a somewhat higher prevalence in males than females. On average, ecstasy users more often lived in urbanized areas and were higher educated than non-users. Ecstasy users were significantly more likely to have used other substances (legal or illegal) compared to non-users. Twin modelling indicated that individual differences in liability to lifetime ecstasy use are for $74 \%$ due to genetic differences between individuals, whereas shared environmental and residual factors explain a small proportion of its liability ( 5 and $21 \%$, respectively). Polygenic risk scores for cannabis use (but not tobacco or alcohol use) significantly predicted lifetime ecstasy use in our sample. This indicates that there is overlap in the genetic vulnerability between use of cannabis and ecstasy.

\section{Unravelling the link between reading ability and print exposure}

Elsje van Bergen, Vrije, Netherlands; Maggie Snowling, University of Oxford, United Kingdom; Eveline De Zeeuw, Vrije Universiteit Amsterdam, Netherlands; Toos Van Beijsterveldt, Vrije Universiteit Amsterdam, Netherlands; Conor Dolan, Vrije Universiteit Amsterdam, Netherlands; Dorret Boomsma, Vrije Universiteit Amsterdam, Netherlands

Purpose: What is the relation between how much children read for pleasure and how well they read? And why are they related at all? Do children who read a great deal become better readers, do good readers spent more and more time on reading, or is a third underlying variable at play?

Method: Partial data were available for a large sample of twin children $(\mathrm{N}=11,408)$ and 389 siblings, all enrolled in the Netherlands Twin Register. Children were assessed around 7.5 years of age. Mothers completed questionnaires on children's reading ability and time spent on reading activities. In addition, information on reading ability was available through teacher ratings and performance on national reading tests. For siblings only test results were available.

Results: Reading ability did not differ between twin and non-twin children, supporting that twin findings can be generalized to the population. For the twin sample, a measurement model was specified with two latent variables, Reading Ability and Print Exposure, which correlated .41. Heritability analyses showed that Reading Ability was highly heritable, while for Print Exposure genetic and environmental influences were equally important. We employed the fact that the two differed in genetic architecture and fitted direction of causality models (see Duffy \&amp; Martin, 1994). These models supported a causative relationship running from Reading Ability to Print Exposure.

Conclusion: How much and how well children read are moderately correlated. Individual differences in print exposure show higher environmental influences than individual differences in reading ability. Importantly, it seems that children's reading ability influences how much they choose to read, rather than vice versa.

\section{References}

Duffy, D.L. \& Martin, N.G. (1994). Inferring the direction of causation in cross-sectional twin data: Theoretical and empirical considerations. Genetic Epidemiology, 11(6), 483-502.

\section{Personality features of women-carriers of different diplotypes on polymorphic loci Val158Met COMT and-uVNTR MAOA}

Elena Vorobyeva, Southern Federal University, Russian Federation; Paver Ermakov, Southern Federal University, Russian Federation; Ekaterina Kovsh, Southern Federal University, Russian Federation; Irina Abakumova, Southern Federal University, Russian Federation

This investigation has been carried out with financial support from RSF (16-18-10222).

The study of the influence of diplotypes genes of neurotransmitter systems on personal characteristics-important topic of modern researches. The aim of the study is to investigate the personality traits of women, carriers of different diplotypes on polymorphic loci Val158Met COMT (GG, GA, AA) and -uVNTR MAOA (H, L, M). Participants: 115 women 18-24 years old. Methods. Genotyping; psychodiagnostics: Buss-Durkee Hostility Inventory, Thomas K.W. test, Questionnaire of formal-dynamic properties of individuality, Diagnostication of personal inclination to deviant behavior. Statistical processing: ANOVA, discriminant analysis, $\mathrm{p} \leq 0.01$. Results. Women with heterozygous diplotype (MAOA M+GA COMT) display low level of aggression and medium intellectual rate; Carriers of diplotype MAOA L+GA COMT are characterized as having high degree of aggression, negativism and communicative plasticity; they also usually display competitive strategies in conflict situations; Women with diplotype MAOA H+GA COMT show low level of aggression, high degree of intellectual ergicity; they can be characterized as conformal; Women with high-activity diplotype (MAOA H+GG COMT) have sanguine temperament; they display high level of aggression, intellectual rate and activity; they show low degree of emotionality in communication, tend to be conformal and do not usually take offence. Carriers of diplotype MAOA M+AA COMT have melancholic temperament, display low level of aggression, slow intellectual rate and decreased activity; they show high emotionality in communication as well as low communicative plasticity; they tend to take offence and behave in non-conformal ways. As seen from stated above, the psychological portraits we describe include antagonistic psychological characteristics. That is why we can suggest that some combinations of genotypes MAOA and COMT might have the mutually opposite impact and that could probably be so due to the peculiarities of neurotransmitter metabolism.

\section{Did I inherit my moral compass?}

Amanda Ramos, The Pennsylvania State University, United States; Amanda Griffin, The Pennsylvania State University, United States; Jenae Neiderhiser, The Pennsylvania State University, United States; David Reiss, Yale University, United States

The development of virtuous character (e.g., social responsibility) has generally been assumed to be due to environmental influences (i.e., social interactions). However, there is support for genetic influences on virtuous character during adolescence and young adulthood. Therefore, studies need to consider how virtuous character can develop not only due to environmental influences (i.e., parenting), but genetic influences to expand how philosophers and researchers understand moral development. Using the Nonshared Environment in Adolescent Development (NEAD; Neiderhiser, J. M., Reiss, D., \& Hetherington, E. M. 2007. The Nonshared Environment in Adolescent Development (NEAD) Project: A longitudinal family study of twins and siblings from adolescence to young adulthood. Twin Research and Human Genetics, 10(1), 74-83), a longitudinal twin/sibling family study $(\mathrm{N}=720$; Time 1 (T1) $\mathrm{M}$ age $=14.8$ for older siblings and 
12.9 for younger siblings, Time 3 (T3) M age $=26.8$ for older siblings and 25.5 for younger siblings), we examined genetic and environmental influences on associations among negative parenting and adolescent self-responsibility at $\mathrm{T} 1$, and virtuous character at $\mathrm{T} 3$. Negative parent-adolescent relationship was comprised of parent-reports on the Parent-Child Relationships scale, Child Rearing Issues, and Conflict Tactics Scale at T1. Self-responsibility was comprised of adolescent and parent report on the Harter and California Personality Inventory at $\mathrm{T} 1$. Virtuous character was assessed by self-report on the NEO Personality Inventory at T3. A trivariate Cholesky decomposition indicated that negative parenting was associated with the development of virtuous character for both genetic and environmental reasons. Specifically, negative parenting was associated with both adolescent self-responsibility and young adult virtuous character due to shared genetic influences, while non-shared environmental influences accounted for associations between negative parenting and adolescent self-responsibility. Finally, genetic influences accounted for associations between adolescent self-responsibility and virtuous character. These findings help to clarify that the correlations among parenting and adolescent and young adult characteristics consistent with virtuous character are largely due to common genetic influences. Therefore, studies that focus only on the "environmental" causes of virtuous character development may miss an opportunity to understand the more complex mechanisms involved.

\section{Genetic influences on risk preference in adolescence: results from a multivariate twin study}

Paige Harden, University of Texas at Austin, United States; Frank Mann, University of Texas at Austin, United States; Natalie Kretsch, University of Miami, United States; Kathrin Herzhoff, Northwestern University, United States; Jennifer Tackett, Northwestern University, United States; Laurence Steinberg, Temple University, United States; Elliot Tucker-Drob, University of Texas at Austin, United States

Risk preference is an important concept in the behavioral sciences that captures individual differences in people's decisions and behaviors in situations involving possible losses and rewards. Risk preferences change with development, and adolescents are particularly likely to engage in risk-taking behaviors. Developmental theories of risk-taking in adolescence have suggested that, rather than being a unitary construct, risk preference actually captures a number of distinct psychological processes. In a diverse sample of 810 adolescent twins and triplets ( $\mathrm{M}$ age $=15.9$ years, $\mathrm{SD}=1.4$ years) from the Texas Twin Project, we investigated the factor structure of 15 selfreport and task-based measures relevant to risk preference in adolescence. Factor analyses suggest that these measures can be organized into four, genetically-separable factors, which we labeled premeditation, fearlessness, cognitive dyscontrol, and reward seeking. Most behavioral measures contained large amounts of task-specific variance; however, most genetic variance in each measure was shared with other measures of the corresponding factor. Behavior genetic analyses further indicated that genetic influences on cognitive dyscontrol overlapped nearly perfectly with genetic influences on IQ $\left(r_{A}=-0.91\right)$, whereas other dimensions of risk preference were largely distinct from IQ. These findings underscore the limitations of using single measures of risk preference in isolation, and indicate that the study of adolescent risk-taking will benefit from applying multimethod approaches. Finally, we discuss how this line of research can be integrated with results from recent genome-wide association studies of risk preference.

\section{Personality traits and low-active diplotype on polymorphic loci Val158Met COMT, -uVNTR MAOA in men}

Ekaterina Kovsh, Southern Federal University, Russian Federation; Paver Ermakov, Southern Federal University, Russian Federation; Elena Vorobyeva, Southern Federal University, Russian Federation

This investigation has been carried out with financial support from Russian Science Foundation (project No. 16-18-10222).

The influence of low-active diplotype (COMT AA, MAOA L) on psychological traits of men is not studied sufficiently, and that is what defined the aim of our work under discussion. Subject: men, aged 1830 years, carrying combination of genotypes of the COMT and MAOA genes (61 people, among them men-carriers of low-active diplotype (COMT AA and MAOA L) - 21 people). Methods. Genotyping; psychodiagnostics: MMPI, Buss-Durkee Hostility Inventory, test of legitimized aggression, Diagnostication of personal inclination to deviant behavior. Statistical methods: Analysis of variance and discriminant analysis, $p \leq 0.01$. Results. In comparison with carriers of different genes combinations, men with combination of MAOA L and COMT AA genotypes display higher levels of physical and verbal aggression, aggressive motivation and higher index of the overall aggression. They show high indices on the integral scale of legitimized aggression, marked inclination to addictive and delinquent behavior and also tend to express aggression and violence freely in the immediate behavior. The sum total of all these indications might testify the highest aggression level in this group. The increase in mean values on the pessimism and paranoia scales (MMPI) might be the evidence of intense anxiety characteristic of this male group and reflect their inclination to depressive and paranoid reactions, hostility and sticking to the negative emotions. The peculiar personality traits of the people with low-active varieties of the genes under study listed above might have destructive influence and contribute to social maladjustment. Judging by the result we get, such people approve of expressing aggression and violence in the society, they are highly inclined to committing illegal actions and try to solve their problems by withdrawal from reality with the help of chemical agents or other, non-chemical addictive behaviors.

\section{Cognitive-behavioral severity in three subtelomeric microdeletion disorders are not related to deletion size}

Gene Fisch, CUNY/Baruch College, United States; Sarah South, University of Utah, United States

Introduction: Chromosomal microdeletions are associated with various behavioral syndromes, intellectual disability (ID) and developmental delay (DD). The phenotype is usually identified first, possibly followed by the discovery of its molecular etiology. Chromosomal microdeletions constitute a small proportion of copynumber variants $(\mathrm{CNVs})$ on the human genome. Microdeletions vary in size and involve one or more genes related to the phenotype. Conventional wisdom in genetics is that disorder severity is associated with the deletion size.

Objective: The aim of this study was to examine the relationship between deletion size and phenotypic severity with respect to cognitive ability and adaptive behavior.

Method: We recruited N=50 children ages 3-20 years, diagnosed with one of three subtelomeric microdeletions: 2q37, 8pinvdupdel, or 11q23-5. All participants were administered a battery of cognitive- 
behavioral tests including the Stanford-Binet 4supth/sup Ed (SBFE) and Vineland Adaptive Behavior Scales (VABS) to assess intellectual ability (IQ) and adaptive behavior (DQ). Of the 50 participants, blood was drawn or buccal smear employed to examine DNA and deletion size in $\mathrm{N}=17$ subjects. Deletion size, IQ and DQ were analyzed using SAS Ver. 9.4.

Results: Results show no significant correlation of deletion size with IQ $(r=-0.17 ; p 0.51)$ or with DQ $(r=-0.10 ; p 0.67)$. Linear regression analysis also indicates no significant decrease in IQ as a function of deletion size (slope parameter $=-1.2 ; \mathrm{p} 0.32$ ), nor was there a significant decrease in DQ $(\mathrm{r}=-0.60 ; \mathrm{p} 0.62)$. Contrary to earlier investigations by researchers who occasionally find significant negative relationships between deletion size and cognitive-behavioral severity, we do not find this to be the case for the 3 subtelomeric deletions we examined, whether the subjects' genetic disorders were analyzed jointly or severally.

Discussion: Results appear to be at variance to the concept of severity in genetic disorders. The concept of severity in behavioral phenotypes will be reconsidered and redefined.

\section{Specificity in etiology of subtypes of bipolar disorder: evidence from a Swedish population-based family study}

Jie Song, Karolinska Institutet, Sweden; Ralf Kuja-Halkola, Karolinska Institutet, Sweden; Arvid Sjölander, Karolinska Institutet, Sweden; Sarah Bergen, Karolinska Institutet, Sweden; Henrik Larsson, Karolinska Institutet, Sweden; Mikael Landén, Institute of Neuroscience and Physiology, The Sahlgrenska Academy at Gothenburg University, Gothenburg, Sweden, Sweden; Paul Lichtenstein, Karolinska Institutet, Sweden

Objectives: The discrete boundaries between current diagnostic classifications of psychiatric disorders have been increasingly challenged by emerging evidences of shared genetic determinants between disorders (1). This challenge is particularly relevant for subtypes of mood disorders. To examine whether bipolar I disorder (BDI) and bipolar II disorder (BDII) have etiological differences, we used a population-based sample to assess the familial aggregation for each subtype and co-aggregation between them. Furthermore, we explored their correlations with schizophrenia, depression, attentiondeficit/hyperactivity disorder, eating disorders, autism spectrum disorders, substance use disorders, anxiety disorders and personality disorders.

Methods: By linking Swedish national registers we established a population-based cohort $(\mathrm{N}=15,685,511)$ and identified relatives with different biological relationships. Odds ratios (ORs) were used to measure the risk of disorders in relatives of individuals diagnosed with BDI $(\mathrm{N}=4,309)$ and BDII $(\mathrm{N}=4,178)$. The heritabilities for BDI and BDII and the genetic correlation across psychiatric disorders were estimated by variance decomposition analysis.

Results: Compared with the general population, the OR of BDI was 17.0 (95\% confidence interval (CI) 13.1-22.0) in first-degree relatives of BDI patients, higher than that of BDII patients (OR 9.8, 95\% CI 7.7-12.5). By contrast, the ORs of BDII were 13.6 (95\% CI 10.218.2) in first-degree relatives of BDII patients and 9.8 (95\% CI 7.712.4) in first-degree relatives of BDI patients. The heritabilities for BDI and BDII were estimated as 0.60 (95\% CI 0.33-0.82) and 0.41 (95\% CI 0.13-0.65), respectively, with a substantial genetic correlation between them $(0.69$, 95\% CI $0.34-0.98)$. The familial coaggregation with other psychiatric disorders, in particular schizophrenia, showed different patterns for BDI and BDII.

Conclusions: Our results suggest a distinction between BDI and BDII in etiology, partly due to genetic differences.

\section{References}

1. Cross-Disorder Group of the PGC, Identification of risk loci with shared effects on five major psychiatric disorders: a genomewide analysis. Lancet. 2013; 381:1371-9.

\section{Parent and offspring polygenic scores as predictors of offspring years of education}

Emily Willoughby, University of Minnesota, Twin Cities, United States; James Lee, University of Minnesota, Twin Cities, United States

A polygenic score (PGS) derived from a 2013 genome-wide association study $(\mathrm{N}=127,000)$ explains $\sim 2 \%$ of the variance in years of education (EduYears). Here, using a PGS derived from a 2016 study $(\mathrm{N}=$ 329,000 ), we tested the association between the PGS and EduYears within families in an independent sample of 2,517 twins and their parents from the Minnesota Center for Twin and Family Research. Our first important result is that within the same DZ twinship, the twin with the higher EduYears PGS is significantly more likely to have a higher IQ $(\mathrm{p}<.01)$ but not a higher EduYears. This is perhaps consistent with the ability of parents to provide educational opportunities to their less able children. We also found that while offspring PGS predicts up to 9\% of the variance in their own EduYears, adding the midparent PGS significantly increases the $\mathrm{R}^{2}$ by about a percentage point. Furthermore, the addition of parental characteristics such as SES, years of education, and IQ to the model diminishes the value of the midparent PGS in predicting offspring EduYears, suggesting that these variables lie on an environmental causal path between parental genotype and offspring EduYears. Interestingly, we observe no such pattern of results when the outcome variable is offspring IQ. That is, while the offspring PGS significantly predicts offspring IQ, the midparent PGS adds no significant increment to the $\mathrm{R}^{2}$. These findings represent the first use of polygenic scores for years of education in clarifying the effects of passive gene-environment correlation on trans-generational outcomes, which may be crucial for disentangling the transactions between genetic and environmental influences on educational attainment.

\section{Effects of occult heterogeneity of binary traits on estimates of genetic variation}

Michael Neale, Virginia Commonwealth University, United States; Kenneth Kendler, Virginia Commonwealth University, United States

Diagnostic criteria may not distinguish between two or more subtypes of a disorder, and these subtypes may have entirely different etiologies. Such 'occult' heterogeneity could systematically affect familial resemblance for a disorder, and lead to biased estimates of genetic or other sources of variation. We explored this possibility using simulation studies, in which two or more independent, equally frequent subtypes exist, any one of which is sufficient to cause the disorder. In the classical twin study, both MZ and DZ correlations are attenuated, but DZ resemblance decreases by a larger proportion, which has the effect of converting additive genetic to non-additive genetic variance. These effects are more pronounced for rarer (e.g., schizophrenia, 1\%) than more common (e.g., depression, 10\%) disorders. We consider different numbers of subtypes, various genetic and environmental correlations between them, and disorder prevalence to understand how hidden subtypes may bias estimates of heritability from studies of twins and genomic assays of individuals with no ostensible family relationship. 


\section{Discrimination and cultural socialization among transracially and internationally adopted adolescents: a longitudinal study}

Samantha Schires, Michigan State University, United States; Nicole T. Buchanan, Michigan State University, United States; Ignacio D. Acevedo-Polakovich, Michigan State University, United States; Richard M. Lee, University of Minnesota, United States; Matt McGue, University of Minnesota, United States; William G. Iacono, University of Minnesota, United States; S. Alexandra Burt, Michigan State University, United States

Despite the increasing prevalence of transracial, international adoption over recent decades, adjustment outcomes for racial and ethnic minority adopted youth placed into White families and the environmental influences of parenting on outcomes are not well understood (Lee, 2006). The current study examined how experiences of racial discrimination affect adjustment outcomes in a sample of internationally and transracially adopted adolescents from the Minnesota Sibling Interaction and Behavior Study (SIBS), and the potential moderating role of racial socialization by parents. Racial socialization refers to the process by which parents teach children about their racial identity and prepare them to cope with experiences of discrimination (Hughes, 1997). Analyses were conducted separately for adolescent internalizing and externalizing problems, using a longitudinal, crosslagged design. Results indicated that, as expected, discrimination predicted higher levels of both internalizing and externalizing problems. However, these associations were moderated by parental racial socialization, such that experiences of discrimination predicted higher levels of adolescent psychopathology only in those adolescents experiencing low levels of racial socialization. In adolescents experiencing high levels of racial socialization, by contrast, the associations between discrimination and psychopathology were notably attenuated and no longer significantly different from zero. Because the adolescents in this study do not share genes with their adoptive parents, the effects of racial socialization cannot be attributed to genetic effects shared between biological family members, and are thus likely to represent an important environmental influence on adolescent outcomes.

\section{Personality similarity in unrelated look-alike pairs: further tests of a twin study criticism}

Nancy Segal, CSU Fullerton, United States; Brittney Hernandez, CSU Fullerton, United States; Jamie Graham, University of Texas at Austin, United States; Ulrich Ettinger, University of Bonn, Germany

The nature of relationships between physical resemblance and personality similarity have generated debate among behavioral science investigators. Research has yielded mixed findings, but positive associations have been advanced by some critics to challenge genetically-based interpretations of personality development. Critics argue that monozygotic (MZ) co-twins' matched physical features invite similar treatment by others, explaining their similar behaviors. Another hypothesis is that MZ co-twins' matched personality traits are largely due to evocative gene-environment correlation-i.e., twins' similar behaviors elicit similar responses from others. To assess these competing explanations, the Personality for Professionals Inventory, Rosenberg Self-Esteem Scale and a social relationship survey were completed by 20 unrelated, look-alike (U-LA) male pairs and 24 U-LA female pairs, Mage $=42.01$ years, $(\mathrm{SD}=16.24)$. This study extends findings by Segal (2013) and Segal, Graham and Ulrich
(2013), based on 23-25 U-LA pairs: correlations were negligible for all five personality factors. In contrast, big five personality correlations are higher for reared-apart MZA $\left(\mathrm{Mr}_{\mathrm{i}}=.53\right)$ and dizygotic (DZA) twins $\left(\mathrm{Mr}_{\mathrm{i}}=.15\right)$. The U-LA correlation was also negligible for selfesteem, compared with the correlations for $\mathrm{MZ}\left(\mathrm{r}_{\mathrm{i}}=.30-.35\right)$ and $\mathrm{DZ}$ reared-together twins $\left(r_{i}=.11-.16\right)$. The criticism that monozygotic co-twins' matched looks explain their similar treatment is refuted in favor of a genetic component underlying twins' personality similarity. Moreover, a smaller proportion of U-LAs expressed close initial and current social relationships than did MZA and DZA twins, consistent with 2013 findings. It is argued that U-LAs do not perceive behavioral similarities in one another that maintain close social relations.

\section{References}

Segal, N.L. (2013). Personality similarity in unrelated look-alike pairs: Addressing a twin study challenge. Personality and Individual Differences, 54, 23-28.

Segal, N.L., Graham, J.L., \& Ettinger, U. (2013). Unrelated lookalikes: A replicated study of personality similarity and new qualitative findings on social relatedness. Personality and Individual Differences, $55,169-176$.

\section{Modeling psychological dynamics with random events}

Kevin McKee, Virginia Commonwealth University, United Kingdom; Michael Neale, Virginia Commonwealth University, United States; Steven Boker, University of Virginia, United States

Background: Cross-sectional measures of affect suffer from interpretive ambiguity and imprecision due to reliance on respondents' retrospective abilities. Causal inferences are limited by their exclusion of temporal information. With state-based measures of affect, respondents only use short-term recall in frequently repeated assessments. Individual traits may be estimated from the statistical properties of resulting time series. If the limitations with trait-based self-assessment are ameliorated by estimating traits from repeated momentary assessments, then trait scores in the latter design should provide more valid and reliable phenotype for use in genetic studies. A wide variety of methods exist for analyzing time series data. General tendencies may include simple means and variances within-individual. With a dynamical systems approach, a latent differential equation (LDE) is estimated with parameters that characterize the damping and frequency of oscillations around equilibrium, given random disturbances to the system $[1,2]$. It is demonstrated that without a method to detect and model each random disturbance or event, parameter estimates are severely biased. Simulations were run to test parameter estimation with and without a novel event-detection algorithm.

Objective: To examine the minimum data requirements for parameter recovery with the Latent Differential Equation model, and to propose additional event-detection methods to improve parameter estimates. Method: Data were simulated under various conditions including different series length, signal/noise ratio, and event probability per time point. The model was fit to 250 randomly generated series per condition to examine the distributions of parameters estimates. The simulations were re-run with a novel event-detection algorithm.

Results: Basic simulations of the Latent Differential Equation model were not robust to multiple disturbances or initial conditions with peaks located at any time point other than the first of the series. Analysis with event-detection corrected parameter bias under a wide variety of conditions, with degrading performance under 3:1 signalto-noise ratio and $\sim 20 \%$ event probability. 


\section{References}

1. Boker, S., Neale, M. \& Rausch, J. (2004). Latent differential equation...indicators. In Recent developments on structural equation models (pp. 151-174). Springer.

2. Chow, S.-M., Ram, N., Boker, S. M., Fujita, F., \& Clore, G. (2005). Emotion...damped oscillator model. Emotion, 5 (2), 208.

\section{The differences in developmental stages in genetic and environmental influences on family social support and depression in late childhood and adolescence}

Mami Tanaka, Chiba University, Japan; Masumi Sugawara, Ochanomizu University, Japan

Depression is the second leading cause of disability in the world (Ferrari et al., 2013) and is the top cause of illness and disability among adolescents (WHO, 2016). Studies have shown the protective role of social support in helping prevent occurrence of depression in adolescence (Dingfelder et al., 2010). In order to prevent depression among children, it is necessary to pay attention to them even before they reach puberty. In this study, we examined the differences in developmental stages in genetic and environmental influences on maternal or paternal support and depression. A total of 530 twins took part in this study, and we divided them into late childhood (age 912 years) and adolescence (age 13-18 years) to compare the two developmental stages. The results of the correlated factor model showed that the magnitude of genetic effect seemed to be more important in adolescence than in late childhood, in the relationship between maternal support and depression. Moreover, the most interesting results were that in late childhood the phenotypic correlation $(\mathrm{r}$ $=-.42, \mathrm{p}<.01$ ) between maternal support and depression was explained by shared common and unique environmental factors $\left(r_{c}=\right.$ -.53 , which explains $60 \%$ of covariation; $r_{e}=-.31$, which explains $40 \%$ of covariation, respectively). In contrast, the phenotypic correlation $(\mathrm{r}=-.47, \mathrm{p}<.01)$ between paternal support and depression was explained by shared genetic $\left(r_{g}=-1.00\right.$, which explains $69 \%$ of covariation) and unique environmental $\left(\mathrm{r}_{\mathrm{e}}=-.25\right.$, which explains $31 \%$ of covariation) factors. The present findings showed that genetic factors had a greater influence than did environmental factors on the relationship between maternal or paternal support and depression in adolescence. In late childhood, however, environmental factors had a greater influence than did genetic factors on the correlations between the variables of maternal support and depression. These results suggest that according to stages of development, it might be necessary to change contents and quality of support provided by mother and father for prevention of children's depression.

\section{Issues in the validation of the general factor of psychopathology}

Irwin Waldman, Emory University, United States; Holly Poore, Emory University, United States; Ashley Watts, Emory University, United States; Paul Rathouz, University of Wisconsin School of Medicine and Public Health, United States; Carol Van Hulle, University of Wisconsin, Madison, United States; David Zald, Vanderbilt University, United States; Benjamin Lahey, University of Chicago, United States

There is increasing interest in a General Psychopathology factor that has been invoked to explain the comorbidity among disorders and symptom dimensions across the Externalizing and Internalizing domains, as well as unresolved issues in the classification and etiology of psychopathology. Despite this enthusiasm, various researchers have questioned the validity and value of the General Psychopathology factor, and the bifactor model that is most often used to characterize it. Using both simulations and real data from 1,568 9-17 year-old twin pairs, we examined several issues in the validation of the General factor of psychopathology. We contrasted the fit and external validity of a bifactor model containing a General and two (i.e., Externalizing, Internalizing) or three (i.e., Externalizing, Fears, Distress) correlated specific factors with a model that only contained the two (or three) correlated factors. We used several criteria to evaluate and compare the validity and utility of these two competing models. In factor analyses, these included various indices of model fit and construct replicability, as well as the sensitivity of the General factor to its constituent first-order symptom dimensions. In external validity analyses, we examined discriminant validity of the factors by characterizing the percent of variance in and patterns of relations with relevant criteria accounted for by the bifactor versus the correlated factors models. In simulations, we examined model fit, statistical power, and parameter bias across the same models. We found evidence for better fit of, and greater discriminant validity in, the bifactor than the correlated factors model and found that the nature of the General factor was largely insensitive to the inclusion of any particular symptom dimension. These validation criteria and our results have implications for the existence and nature of the General psychopathology factor and for evaluating models of the classification of psychopathology in general.

\section{Analysis of genetic similarity among friends and schoolmates in the National Longitudinal Study of Adolescent to Adult Health (Add Health)}

\section{Ben Domingue, Stanford University, United States}

It has been long known that human relationships are genetically stratified. Whether genetic similarity among those in a relationship is due to complex ancestral patterns linked to historical migration, macro-level social structures in modern society, or individual-level peer selection remains unsettled. We use data from 9,500 adolescents from the National Longitudinal Study of Adolescent to Adult Health to examine genetic similarity among school-based friends. While friends have correlated genotypes, both at the whole-genome level as well as at traitassociated loci (via polygenic scores), the results suggest that macrolevel forces, such as school assignment, are a prime source of genetic similarity between friends. Further, we find evidence consistent with the existence of social genetic effects as an individual's educational attainment is highly associated with the polygenic scores of those in their broader social environment (e.g., school) and of their friends (net of their own score). In contrast, individual BMI and height are largely unassociated with the genetics of peers.

\section{The application of ecological momentary assessment and geolocation to a longitudinal twin study of substance use}

David Brazel, University of Colorado Boulder, United States; Robin Corley, University of Colorado Boulder, United States; Chanda Phelan, University of Michigan, United States; Maia Frieser, University of Colorado Boulder, United States; Hariharan Subramonyam, University of Michigan, United States; Sally-Ann Rhea, University of Colorado Boulder, United States; Helen Vernier, University of Colorado Boulder, United States; John Hewitt, 
University of Colorado Boulder, United States; Paul Resnick, University of Michigan, United States; Scott Vrieze, University of Colorado Boulder, United States

Substance use and dependence are a set of heritable behaviors that have a very significant impact on global morbidity and mortality. (1) Adolescence is a sensitive and high-risk period for substance experimentation, use, and dependence. Early substance use in adolescence greatly increases the risk of addiction for an individual. (2) The Colorado Online Twin Study (CoTwins) aims to expand our understanding of the genetic and environmental influences on adolescent substance use. CoTwins consists of 335 twin pairs, between the ages of 14 and 18 at enrollment, who underwent both in-person and remote internet-based ecological momentary assessments. The in-person assessments consisted of standard measures of substance use behavior, parental monitoring, cognitive ability, executive function, and other relevant traits. The remote assessments were administered with smartphone apps and web browser extensions, installed on the twins' devices during the in-person assessment. The apps and the extension were used to regularly administer questionnaires regarding substance use and related factors. They were also used to monitor social media use, web browsing activity, and mobile app usage. Smartphone GPS sensors were used to track the twins' physical location over time. To date, we have logged more than 3.5 million participant locations and will present results demonstrating that the locations are accurate, heritable, and hint at substantial sex-limitation. The twins have completed a total of 50,280 remote assessments, which show a high concordance with the in-person assessment results. Finally, we will present analyses that relate the location data to standard measures of parental monitoring and that explore the relationship between twin geographic distance and phenotypic similarity.

\section{References}

1. Ezzati, M., Lopez, A. D., Rodgers. A., Vander-Hoorn, S., \& Murray, C. J. L. (2002). Selected major risk factors and global and regional burden of disease. Lancet, 360, 1347-1360.

2. Jordan, C. J., \& Andersen, S. L. (2016). Sensitive periods of substance abuse: Early risk for the transition to dependence. Developmental Cognitive Neuroscience.

\section{Personality change in late adulthood: a reciprocal effects modeling approach}

Christopher Beam, University of Southern, United States; Emily Sharp, Yale University, United States; Chandra Reynolds, University of California, Riverside, United States; Margaret Gatz, University of Southern California, United States

Personality traits have been found to undergo changes across the lifespan (Roberts et al., 2006). Numerous developmental behavioral genetic studies have decomposed stable and measurement-specific variability in personality into independent genetic and environmental components to understand whether genetic influences, environmental influences, or both account for stability and change in personality (Briley \& Tucker-Drob 2014), yet no longitudinal twin study to date have explicitly correlated genetic and environmental components that might underlie personality change. Further twin models that assume independence of genetic and environmental components may missrepresent real developmental processes underlying personality development, as these models misspecify how genetic and environmental influences correlate over real time (Beam \& Turkheimer, 2013). In this study we tested whether the reciprocal exchange between people and their environments induces er gene-environment correlation as people age. We present a longitudinal twin study of personality development (neuroticism, extraversion, and openness to experience) using samples of MZ and DZ twins from the Swedish Adoption/Twin Study of Aging (SATSA; Finkel \& Pedersen, 2004) from 1984 to 2010 (MZ pairs=589; DZ pairs=1194). We specified a genetic simplex model that includes parameters that model the reciprocal exchange between DZ twins and their unique environments. The parameter allows for the accrual of within-family geneenvironment correlation in DZ twins, which may explain why DZ twins' personality scores diverge more rapidly than MZ twins' scores. Results suggest that within-family gene-environment correlation does not explain why DZ twins' personality scores diverge over time. Within-family differences in personality development appear to be attributed to unique environmental factors. We comment on the conditions under which including phenotype-environment parameters should be tested in longitudinal twin models.

\section{Genetic risk shared between autism and schizophrenia}

Elizabeth Schafer, University of Illinois at Urbana-Champaign, United States; Jaime Derringer, University of Illinois at UrbanaChampaign, United States; Howard Berenbaum, University of Illinois, at Urbana-Champaign, United States

Prior research demonstrating phenotypic and genotypic overlap between schizophrenia and autism ( $\mathrm{rg}=0.14$, Bulik-Sullivan et al., 2015, Archives Gen Psychiatry) has contributed to an increasing awareness of shared etiology for these disorders (Craddock \& Owen, 2010, British J Psychiatry). We applied the Cross Phenotype Association (CPASSOC) R package (Zhu et al., 2015, Am J Human Genet) to summary statistics from the Psychiatric Genomics Consortium (Schizophrenia Working Group, 2014, Nature; Cross-disorder Group, 2013, Lancet) to identify independent SNPs significantly associated with both disorders, as well as implicated genes and pathways. These findings have implications for shared etiology in schizophrenia and autism and may suggest potential alternative classifications for subtypes of each. More broadly, this study also builds upon previous efforts to examine the feasibility of cross-phenotype meta-analysis for maximizing GWAS sample size for complex or uncommon outcomes.

\section{Nighttime and daytime sleep. An exploration into the genetic and environmental factors for "siesta"}

Juan Ordonana, University of Murcia, Spain; Jesus Lopez-Minguez, University of Murcia, Spain; Lucia Colodro Conde, QIMR, Australia; Eduvigis Carrillo, University of Murcia, Spain; Jose A. Madrid, University of Murcia, Spain; Marta Garaulet, University of Murcia, Spain

Introduction: Siesta is a relevant aspect of sleep due to its possible relation to important aspects of human life, such as health or cognitive functioning. However, unlike nighttime sleep, studies about daytime sleep determinants and characteristics are scarce, and the genetic and environmental structure of siesta is still unknown. Our main objective was to explore the relative contribution of genetic and environmental factors to variation in the sleep-wake rhythm as measured by a continuous assessment of temperature, activity, and position (TAP), which allows for analysis of diurnal sleep.

Methods: The sample comprised 53 pairs (28 MZ and $25 \mathrm{DZ})$ of female twins selected from the Murcia-Twin Register (MTR). Mean age of participants was 52 (SD: 6) years. Zygosity was determined by DNA. We conducted separate univariate analyses to study the sources of variance of daytime and nighttime sleep parameters. 
Results: About $60 \%$ of the sample reported to take siesta at least once a week. Daytime sleep duration was positively associated to anthropometric measures (BMI, total body fat and visceral fat). Heritability of self-reported siesta taking was .65 (95\% CI: 0.19, 0.92). Sleep parameters assessed through continuous monitoring of TAP, including daytime and nighttime sleep duration, showed heritability estimates between 0.36 (95\% CI: 0, 0.68) and 0.69 (95\% CI: 0.38 , 0.85 ), suggesting a relevant impact of genetic factors on the sleep rhythm.

Conclusions: This is the first study to investigate the relative contribution of genetic factors to siesta. By using an integrative measure (TAP) we have been able to introduce a novel approach to the study of diurnal sleep characteristics. Results represent a significant step in the understanding of siesta and daytime sleep nature that are relevant to assess the chronobiological system condition and its possible association with pathologies such as obesity.

\section{Effect of age on the heritability of sleep quality}

Juan J. Madrid-Valero, University of Murcia, Spain; Jose J Morosoli, University of Murcia, Spain; Juan F. Sanchez-Romera, University of Murcia, Spain; Leticia Serrano-Belmonte, University of Murcia, Spain; Jose M. Martinez-Selva, University of Murcia, Spain; Juan Ordonana, University of Murcia, Spain

Introduction: Poor sleep quality has important consequences for psychological well-being. The quality of sleep has an important genetic influence. Heritability estimates between 34 and $43 \%$ have been reported in middle-aged and young adults, respectively. On the other hand, age has a fundamental influence on this phenotype. Sleep quality deteriorates markedly with age and most of the sleep problems usually increase their prevalence and intensity after adulthood. However, little is known about the variation in genetic influences on sleep quality due to age. Our objective is to analyze the role of age as a possible moderating factor of the heritability of sleep quality.

Method: Sample comprise 1912 subjects (55.9\% female), aged 4371 years $($ mean $=53.4)$, participating in the Murcia Twins Registry (644 monozygotic and 1268 dizygotic). Sleep quality was measured using the Pittsburgh Sleep Quality Index. Heritability estimation and moderation analysis were performed using structural equation models.

Results: Heritability of sleep quality in the total sample was estimated at 34\% (95\% CI: $24-43 \%$ ), with a significant effect of genetic factors (AE model). No significant effect of shared environmental factors was observed. Age moderated the relative impact of genetic and environmental factors, such that the importance of non-shared environmental factors (E) was significantly reduced in older women. Consequently, heritability increased up to $85 \%$ in this age group. No similar effect was found in males.

Discussion: Our results are consistent with previous studies that find a moderate genetic influence on sleep quality in the total sample. However, the relative importance of genetic factors appears to increase with age. These results provide new information on the evolution of the differences in sleep quality, between men and women, in adulthood.

\section{Initiation of cannabis use during the 1980s in Spain. Social perception and heritability}

Jose J. Morosoli, University of Murcia, Spain; Laura Mezquita, University Jaume I, Spain; Manuel I Ibañez, University Jaume I, Spain; Lucia Colodro Conde, QIMR, Australia; Generos Ortet, University Jaume I, Spain; Cintia Serrano-Belmonte, University of Murcia, Spain; Francisca Gonzalez-Javier, University of Murcia, Spain; Juan Ordonana, University of Murcia, Spain

Introduction: Environmental factors, like social perception or tolerance, may influence the heritability of recreational drug use. Such heritability would increase or decrease depending on the role of environmental factors in inter-individual variability. The analysis of the heritability of initiation and maintenance in the consumption, in key moments related to the social perception of this consumption, can shed light on this question. Our objective is to analyze the relative contribution of genetic and environmental factors at the initiation of cannabis use in a sample of young twins at the time of the massive introduction of cannabis in Spain (1980s).

Method: The sample comprised 1609 subjects (55.0\% female), aged between 47 and 73 years (mean=56.8; SD: 7.1), participating in the Murcia Twin Registry (564 monozygotic and 1045 dizygotic). Information on cannabis initiation and current use was collected through a telephone interview. Heritability estimation was performed using structural equation models.

Results: $14.6 \%$ of the sample had ever used cannabis. The model fitting suggests that twin resemblance for initiation in cannabis use is due mainly to genetic factors (AE model), with an estimated heritability of $62.4 \%, 95 \%$ CI [44.2, 76.6].

Discussion: The heritability of initiation in cannabis use was high in restrictive social circumstances but with favorable pressure on the part of the reference group. The effect of shared environmental factors is undetectable under such conditions.

\section{Combining multiple genetic risk scores with social environmental factors in explaining childlessness}

Renske Verweij, University of Groningen, Netherlands; Harold Snieder, University Medical Centre Groningen, Netherlands; Gert Stulp, University of Groningen, Netherlands; Melinda Mills, University of Oxford, United Kingdom; Nicola Barban, University of Oxford, United Kingdom; Felix Tropf, University of Oxford, United Kingdom; Guang Guo, University of North Carolina, Chapel Hill, United States

Using GWAS meta-analysis we previously found genes for number of children ever born (NEB) and age at first birth (AFB). Based on these results, we created polygenic risk scores (PGS) and found that genes related to AFB have an impact on childlessness in a Swedish sample. In the current study we extend these findings by examining the relative importance of, and interplay between, the genetic and social environmental influences in predicting childlessness. We take into account multiple PGS for fertility-related traits in addition to modelling social environmental factors using data from men and women from the Health and Retirement Study $(\mathrm{N}=12,205)$. We created PGS for NEB and AFB and will do the same for endometriosis, age at menopause, polycystic ovary syndrome, azoospermia, oligozoospermia and testicular dysgenesis syndrome. We further include the influence of marital status, educational and occupational behavior and birth cohort. Initial results show a positive relationship between the AFB PGS and childlessness: the odds of remaining childless are around 1.18 times higher for women and 1.14 times higher for men with a 1 SD higher score on the AFB PGS. About half of this effect is mediated by education, meaning that these genes influence educational attainment which affects childlessness. In line with twin heritability studies suggesting higher heritability of AFB in more recent cohorts, we find that the PGS also have a higher effect on childlessness in more recent birth cohorts $(\mathrm{p}=.0117)$ and is higher for women with low and relatively high educational attainment $(\mathrm{p}=.0089$ $\& \mathrm{p}=.002)$. In the next analytical steps we will add the individual and 
combined effects of the PGS for the other fertility-related traits on childlessness to our models and results will be presented at the conference.

\section{Genetic heterogeneity in neuroticism}

Mats Nagel, VU, Netherlands; Sven Stringer, VU Amsterdam, Netherlands; Kyoko Watanabe, VU Amsterdam, Netherlands; Sophie van der Sluis, VU, Netherlands; Danielle Posthuma, VU Amsterdam, Netherlands

Genome-wide association (GWA) studies have revolutionized the field of genetics and identified many genetic variants associated to complex traits. However, a large part of the phenotypic variance remains unexplained. The limited yield is often attributed to traits being genetically highly complex (i.e., many variants are involved, each of small effect), and GWA studies may be underpowered to robustly detect very small genetic effects. An alternative explanation, however, can be found in how complex traits are operationalized in genetics studies. Especially in studies on traits like depression, personality and schizophrenia trait measures often consist of composite scores (e.g., case-control status, sum scores), aggregating information from many different symptoms (e.g., in depression: irritable mood, insomnia, worrying, concentration problems, and suicidal ideation). If the aggregated symptoms are genetically heterogeneous, item-specific genetic signals are highly diluted when composite scores are analyzed. In a large population sample (UK biobank; N 113,000), we studied in detail the genetic heterogeneity of 12 neuroticism items, which correlate highly with depression. GWA analyses were conducted on the 12 items separately, and their sum. To assess genetic heterogeneity, we (1) estimated the SNP-based heritability of individual items, (2) established genetic correlations among the items and with external variables, and (3) examined the overlap in associated SNPs and genes across items. We identified 19 independent genomic loci that reached genome-wide significance (GWS) at $\mathrm{P}<5 \times 10^{-8}$. Eight of these loci were only significant for (one or more) individual items, and not for the sum score. Six loci reached GWS for the sum score but not in individual items. Inter-item genetic correlations ranged from 0.31 to 0.92 , suggesting substantial genetic heterogeneity. In addition, genetic correlations between neuroticism items and a number of external traits (e.g., BMI, IQ, bipolar disorder) showed variation in both strength and direction. Our findings suggest that symptom-specific analyses can increase the genetic signal and yield novel biological information. Moreover, the variants identified through the use of a composite score may not be representative of all (or even the majority) of the individual items.

\section{Characterising the transcriptome in autism spectrum disorder using RNAseq}

Emma Meaburn, Birkbeck College, University of London, United Kingdom; Ayden Saffari, Birkbeck College/London School of Hygiene and Tropical Medicine, United Kingdom; Frank Dudbridge, Leicester University, United Kingdom

Autism spectrum disorder (ASD) is a common developmental disorder typified by deficits in social communication and stereotyped behaviours. Despite significant progress in identifying genetic risk variants, there is currently no unified pathomechanistic explanation. Monozygotic (MZ) twins show incomplete concordance in autistic traits, which suggests that alternative risk pathways involving nonshared environmental (NSE) factors could also have an important role to play. In this study we performed RNA sequencing of whole blood and characterised genome-wide gene expression in a sample of 23 ASD MZ twin pairs that were concordant for ASD ( $\mathrm{N}=6$ pairs), discordant for ASD $(\mathrm{N}=6)$ and concordant unaffected controls $(\mathrm{N}=$ 11). We firstly compared ASD affected with unaffected, age-matched controls across the sample (between-group comparison) in order to identify genes and pathways commonly disrupted in ASD. Secondly, we performed within-group and within-pair analyses of the discordant twin pairs in order to identify genes and pathways disrupted by environmental factors.

As expected, fold changes were modest but we identified a number of genes that were differentially expressed $(\mathrm{FDR}<0.2)$ in both the between group analysis (DEPDC1B, IGHG4 and ZNF501) and within group analysis (IGHG4, EVI2A, SNORD15B, RGS18, LPAR6 and RPL9). Geneset testing using ROAST and ROMER methods found no evidence for enrichment of previously identified ASD risk genes, but identified a number of potentially relevant MSigDB pathways $(\mathrm{p}<.05)$, including those related to transcriptional control, immune system function and cellular signaling. While none of the genes identified in this study are have been previously established as ASD susceptibility genes, immune system disruption is one of the most consistent findings in transcriptome studies of ASD. Replicating the immune-related genes identified here for further study in larger discordant twin cohorts is a research priority, where we then might begin to investigate links to environmental exposures.

\section{The relationship between psychotic experiences and regular smoking during adolescence: a genetically sensitive design}

Wikus Barkhuizen, Birkbeck College, University of London, United Kingdom; Angelica Ronald, Birkbeck College, University of London, United Kingdom

Background: High rates of smoking among individuals with psychiatric disorders is well documented. Few studies have investigated an association between smoking tobacco and psychotic experiences (PEs) in non-clinical samples. Of those that have, none considered the full spectrum of PEs while controlling for cannabis use. This study aimed to investigate whether tobacco predicts specific PEs over and above cannabis use, and to estimate the genetic and environmental contributions to regular smoking in healthy adolescents.

Methods: The Specific Psychotic Experiences Questionnaires was used to assess 3,787 twin pairs from the Twins Early Development Study. Twins reported on substance use and PEs including paranoia, hallucinations, grandiosity, cognitive disorganisation and anhedonia. Parents reported on the twins' negative symptoms. Multiple linear regression models were conducted to control for cannabis use. Univariate liability threshold structural equation model-fitting was conducted on regular versus non-regular smokers to estimate the contributions of additive genetic (A), shared environmental (C) and unique environmental (E) influences.

Results: Tobacco and cannabis use both significantly predicted paranoia $(\beta=17.40, \mathrm{t}(3527)=4.13, \mathrm{p}<.001)$, hallucinations $(\beta=7.22, \mathrm{t}$ $(3531)=2.98, p=.003)$ and cognitive disorganisation $(\beta=4.40, t(3530)$ $=4.01, \mathrm{p}<.001)$. In contrast, only tobacco, and not cannabis, predicted negative symptoms $(\beta=5.78, \mathrm{t}(3507)=3.80, \mathrm{p}<.001)$ and anhedonia $(\beta=19.84, \mathrm{t}(3528)=6.49, \mathrm{p}<.001)$. Twin modelling indicated an ACE model provided the best fit $(\mathrm{BIC}=-36824.12$; AIC $=-8168.68)$, with estimates of $\mathrm{A}, \mathrm{C}$, and $\mathrm{E}$ of 37,54 and $9 \%$, respectively.

Conclusions: These findings cast further light on the relationship between tobacco and PEs, indicating that tobacco is associated with a range of non-clinical PEs. These results have implications for developmental theories on the role of substance use in early risk for 
psychiatric disorders. Further analyses should explore the degree to which phenotypic causality, and overlapping genetic and environmental influences, can explain the association between specific PEs and regular smoking.

\section{The more the merrier? Advantages of multivariate genetic association analyses}

Cesar Vroom, VU Medical Center, Netherlands; Christiaan De Leeuw, VU Amsterdam, Netherlands; Danielle Posthuma, VU Amsterdam, Netherlands; Conor Dolan, Vrije Universiteit Amsterdam, Netherlands; Sophie van der Sluis, VU, Netherlands

In Genome-Wide Association Studies (GWAS) researchers typically test the association between millions of Single Nucleotide Polymorphisms (SNPs) and a single trait. Data sets, however, often contain data on multiple phenotypes that may be genetically and phenotypically correlated. In addition, many phenotypes that are treated as a single trait in GWAS may actually be multivariate in nature (e.g., multiple, possibly genetically heterogeneous, symptoms underlie univariate case-control status variables). To accommodate multiple correlated phenotypes in GWAS analyses, various multivariate methods are available. The validity and power of some of these methods have been studied using simulation, but simulation scenarios are often limited (e.g., including only three homogeneously correlated phenotypes) and not comparable over studies. As a result, a thorough comparison of different methods, and a clear overview on which methods perform best under which circumstances, is as of yet not available. In the present study, we simulated data under 270 different realistic scenarios. The simulation scenarios consist of either 1 - or 2 -factor models, with 4,8 , or 16 observed variables. We varied both within and between factor correlations, the location of the genetic effect (i.e., either on 1, half, or all variables), the sign of the genetic effect (i.e., congruent or opposite across variables), and the effect sizes. We then compared the statistical power of three different classes of multivariate methods: reduction-based methods (e.g., factor analysis, PCA, CPC), regression-based methods (e.g., MANOVA, LME, GEE), and combination tests (e.g., TATES, adjusted Fischer Combination test, JAMP). For comparison with the traditional univariate approach we also included the regression on the sum of all variables (i.e., sum score), and the regression on a single affected observed variable. In addition, we conducted extensive simulations (20 scenarios, 1 million simulations per scenario) to investigate the false positive rate of all included methods for increasingly stringent alpha levels (0.05-0.00001). Our results show that the false positive rate of some methods is off for the lower alpha levels that characterize current GWAS. In addition, the power to detect genetic variants varies wildly across methods and over scenarios, which complicates a general prioritization of multivariate methods.

\section{The manifestation of genetic risk for psychiatric disorders in a general population sample of Swedish twin children}

Joanna Martin, Karolinska Institutet, Sweden; Mark Taylor, Karolinska Institutet, Sweden; Lu Yi, Karolinska Institutet, Sweden; Isabell Brikell, Karolinska Institutet, Sweden; Sebastian Lundström, University of Gothenburg, Sweden; Paul Lichtenstein, Karolinska Institutet, Sweden
Psychiatric disorders are complex phenotypes. Molecular genetic studies have implicated numerous common risk variants that are, to a large extent, shared across different disorders. Recent studies suggest that genetic risk variants for psychiatric disorders manifest as early childhood behavioural problems across a number of domains that are not always clearly associated with the psychiatric disorder (e.g. schizophrenia genetic risk is associated with social understanding difficulties and irritability at 7-9 years (Riglin et al. 2016)). We aimed to comprehensively test for association between genetic risk for 8 psychiatric phenotypes with a range of behavioural and psychiatric (e. g. neurodevelopmental and affective) phenotypes.

Genome-wide association study (GWAS) summary statistics for 8 psychiatric phenotypes were used to derive polygenic risk scores (PRS) in a Swedish population of twins ( $N=13,472$ individuals) with data available at ages 9, 12, 15 and 18 years (Child \& Adolescent Twin Study in Sweden). We tested for association between psychiatric disorder PRS with population traits (assessed using parent- and self-report questionnaires) and presence of psychiatric disorders (assessed through linkage with the Swedish National Patient Registry) in the sample. After accounting for multiple testing, the results showed numerous significant associations across phenotypes. Schizophrenia PRS were associated with traits of attention-deficit/ hyperactivity disorder (ADHD), autism, tics, obsessive-compulsive disorder (OCD), anxiety and depression. Bipolar disorder PRS were associated with autism traits. Depression PRS were associated with ADHD and OCD traits and diagnosed anxiety. OCD PRS were associated with anxiety traits and diagnosed autism and depression. ADHD PRS were associated with neurodevelopmental and affective (depressive and manic) problems. Autism PRS were associated with ADHD traits. Tic disorder and anxiety PRS showed no association with other phenotypes. This study provides further evidence for shared genetic risks across different clinical phenotypes.

\section{References}

Riglin, L. et al., 2016. Schizophrenia risk alleles and neurodevelopmental outcomes in childhood: a population-based cohort study. The Lancet Psychiatry.

\section{Assessing the potential shared genetic aetiology between body mass index (BMI) and sleep duration}

Victoria Garfield, University College London, United Kingdom; Ghazaleh Fatemifar, University College London, United Kingdom; Caroline Dale, University College London, United Kingdom; Yanchun Bao, University of Essex, United Kingdom; Melissa Smart, University of Essex, United Kingdom; Andrew Steptoe, University College London, United Kingdom; Clare Llewellyn, University College London, United Kingdom; Delilah Zabaneh, King's College, United Kingdom; Meena Kumari, University of Essex, United Kingdom

Observational studies find an association between body mass index (BMI) and self-reported sleep duration in adults such that a higher BMI is associated with shorter duration of sleep. However, the key question here is whether this relationship is causal, in either direction. Recently, findings from a Mendelian randomisation study in the UK Biobank suggest that a higher BMI does not in fact cause short sleep duration. Thus, it is likely that there are alternative biological pathways through which BMI and sleep duration are associated, but this has not been examined in depth, to date. To investigate this further we created several polygenic risk scores (PRSs) of BMI and examined their association with self-reported sleep duration in a combination of individual participant data (IPD) and summary-level data, with a total 
sample size of $\sim 140,000$. We find that, although a PRS of BMI is negatively associated with sleep duration, this explains a very small proportion of its variance. We will present detailed findings alongside a discussion of these, with reference to previous research and potential for future work.

\section{SES-of-origin and BMI in youth: comparing Germany and Minnesota - to be part of symposium 'gene $x$ environment interplay on indicators of social inequality'}

Wendy Johnson, University of Edinburgh, United Kingdom; Elisabeth Hahn, Saarland University, Germany; Juliana Gottschling, University of Saarland, Germany; Franziska Lenau, University of Saarland, Germany; Frank M. Spinath, Saarland University, Germany; Matt McGue, University of Minnesota, United States

BMI consistently shows considerable genetic but also shared environmental variance across the lifespan, the latter particularly at younger ages. Several studies to date have observed less total and genetically influenced variance with higher attained SES in adults, but we are unaware of analogous studies of SES-of-origin in youth samples. Moreover, there is no reason to expect the same proportions of genetic and environmental influences and moderating effects of SES in different countries with different health policies, lifestyles, and degrees of social inequality. We examined SES-of-origin moderation of BMI variance in the German TwinLife study's four birth cohorts, ranging in age from 5 to 25, and in the Minnesota Twin Family Study's two birth cohorts at ages 11,17 , and 24, comparing males and females separately. In general we observed much higher SES moderation of environmental than genetic influences, in contrast to studies of attained-SES moderation of BMI in adults. We discuss between-country and between-sex differences in our SES-of-origin observations, interpreting them in light of the inevitably-missing covariance between SES-of-origin and BMI in our models, mother-father and parent-offspring BMI correlations, and analogous attained-SES observations in adult samples.

\section{Equivalence of $\mathrm{LD}$-score regression and individual- level-data methods}

Ronald de Vlaming, VU, Netherlands; Magnus Johannesson, Stockholm School of Economics, Sweden; Patrik K. E. Magnusson, Karolinska Institutet, Sweden; M. Arfan Ikram, Erasmus Medical Center, Netherlands; Peter M. Visscher, University of Queensland, Australia

LD-score (LDSC) regression disentangles the contribution of polygenic signal, in terms of SNP-based heritability, and population stratification, in terms of a so-called intercept, to GWAS test statistics. Whereas LDSC regression uses summary statistics, methods such as HasemanElston (HE) regression and genomic-relatedness-matrix restricted maximum likelihood (GREML) estimation infer SNP-based heritability from individual-level data directly. Owing to the difference in data used by these two types of methods, they are typically considered to be different classes of methods. Nevertheless, recent work has already revealed that LDSC and HE regression yield approximately equivalent estimates of SNP-based heritability when confounding stratification is absent. We extend the equivalence; under the stratification assumed by LDSC regression, we show that the LDSC intercept can be estimated by performing a simple regression of the phenotype on the leading principal component from the genomic-relatedness matrix and transforming this estimate using the corresponding eigenvalue. Using simulated phenotypes, we find in case of two discrete populations that intercept estimates obtained from individual-level data are nearly equivalent to estimates from LDSC regression itself $\left(\mathrm{R}^{2}=99.9 \%\right.$ between estimates from different methods). Moreover, we show using three discrete populations that the intercept, as estimated by LDSC regression, can be retrieved nearly perfectly from a linear combination of squared coefficients of a regression of the phenotype on the leading two principal components, weighted by a function of the corresponding eigenvalues and sample size $\left(\mathrm{R}^{2}=99.1 \%\right.$ between estimates). An empirical application corroborates these findings. Hence, an equivalence principle holds even for complex forms of stratification. Consequently, methods such as LDSC regression are not profoundly different from methods using individual-level data; parameters that are identified within the LDSC framework can be identified equally well by methods using individual-level data.

\section{Do genetic factors explain the link between language and school readiness in toddlers?}

Kayla Finch, Boston University, United States; Megan Flom, Boston University, United States; Kimberly Saudino, Boston University, United States

The positive association between language skills and school readiness has been well established (Margo et al., 2011). Early vocabulary and later school readiness both show small, but significant, heritability with shared and nonshared environmental influences contributing the most to their variances (Foget-Dubois et al., 2009). Moreover, the covariance between vocabulary and school readiness are due only to environmental (shared and nonshared) influences. However, this research relied on parent-report to assess vocabulary, which may have inflated shared environmental influences due to rater biases. To date, no study has examined the early relation between language and school readiness assessed through behavioral assessments. We investigated the genetic and environmental contributions to individual differences in vocabulary and school readiness and their overlap in 3-year-old twins (123 MZ, 189 DZ). School readiness was measured using the Bracken School Readiness Assessment (BSRA; Bracken, 2000) and receptive vocabulary was measured using the NIH Toolbox Picture Vocabulary Test (PVT; Gershon et al., 2013). We found a moderate phenotypic correlation between the BSRA and PVT $(r=.38$, $\mathrm{p}<.05)$. Cholesky model fitting analyses showed genetic influences accounting for $29 \%$ (CI 9-51\%) of the variance for the BSRA and $31 \%$ (CI 6-55\%) for the PVT. Shared environment influences were significant for the BSRA (41\%; CI 20-57\%), but not for the PVT (14\%; CI 0-35\%). The remaining variance for BSRA and PVT was explained by nonshared environmental influences. Overlap between BSRA and PVT was explained entirely by genetic effects, and these genetic effects overlapped completely $\left(\mathrm{r}_{\mathrm{G}}=1.00\right.$; CI $\left.0.49-1.00\right)$. Unlike previous findings, we found that the relation between school readiness and language abilities was due to shared genetic influences and not environmental influences. This could be due to the different measures used in assessing language as our current study relied on behavioral assessments and not parent-report.

\section{Social and genetic factors influencing human fertility behavior}

Jornt Mandemakers, Utrecht University, Netherlands

I will present on the interplay between genetic and social factors in human reproductive behavior. First, I will introduce our recent 
findings on the role of genes in human reproductive behavior (GWAS). Second, I will illustrate how genetic designs can help to get a better understanding of the social determinants of reproductive behavior. See abstract below.

\section{Education and postponement of fertility}

A large body of literature shows a positive relationship between education and age at first birth. However, this relationship may in part be spurious due to family background factors that cannot be controlled for in most research designs. We investigate to what extent education is causally related to later age at first birth in a large sample of female twins from the UK $(\mathrm{N}=2,752)$ and a large sample of siblings in the Netherlands $(\mathrm{N}=32,000)$. For the $\mathrm{UK}$, we present novel estimates using within-identical twin and biometric models. Our findings show that one year of additional schooling is associated with about half a year later age at first birth in OLS models. This reduced to only 1.5 months for the within-identical twin model that controls for all shared family background factors (genetic and family environmental). Biometric analyses reveal that it is mainly influences of the family environment-not genetic factors-that cause spurious associations between education and age at first birth. Lastly, we demonstrate using data from the Office for National Statistics that only 1.9 months of the 2.4 years of fertility postponement for birth cohorts 1944-1969 could be attributed to educational expansion based on these estimates. We conclude that (the rise in) educational attainment alone cannot explain differences in fertility timing (between cohorts). The results for the Dutch sample using siblings yields similar results.

\section{The relationship between genes, cognitive and personality traits, and political participation}

Christopher Dawes, New York, United States; Sven Oskarsson, Uppsala University, Sweden; Ben Domingue, Stanford University, United States; Aysu Okbay, Free University Amsterdam, Netherlands

Several recent twin studies have demonstrated that political participation can be explained in part by genetic variation. Further, there is evidence suggesting that cognitive ability and personality traits mediate the relationship between genes and political participation. In this study, we construct polygenic scores for cognitive (educational attainment and verbal ability) and personality (extraversion and neuroticism) traits that are predictors of individual differences in political participation in order to test whether these traits are pathways through which genes may indirectly influence political participation. We conduct our analysis based on two US samples (Minnesota Twin and Family Study and the National Longitudinal Study of Adolescent Health) and one Swedish sample (Swedish Twin Registry). We find that each of the polygenic scores are significantly associated with political participation, however the highest relationship is between educational attainment and political participation. Interestingly, the associations we report are not entirely mediated by cognitive and personality traits.

\section{Links between callous-unemotional behaviors and prosociality in early childhood}

Megan Flom, Boston University, United States; Kimberly Saudino, Boston University, United States

In middle childhood through adolescence, callous-unemotional traits (CU) and prosociality are negatively correlated (e.g., Roose et al.,
2010). Despite the conceptualization of CU as a disruption of the moral behaviors necessary for prosocial development, many correlations tend to be moderate, highlighting that prosociality and $\mathrm{CU}$ are not simply opposites. Nothing is known, however, about how the two are related in early childhood, or the shared genetic and environmental etiology at any point in development. The present study explored the underlying genetic and environmental overlap between CU and prosociality in 309 twin pairs (123 MZ, 186 DZ) at ages 3 and 4 years, when these moral behaviors are emerging. CU was assessed using the Child Behavior Checklist 1.5-5 (Achenbach \& Rescorla, 2000), and prosociality using the Strengths and Difficulties Questionnaire (Goodman, 1997). CU ( $\mathrm{r}=.46)$ and prosociality $(\mathrm{r}=.53)$ were stable across age; and correlated within age (age 3: $r=-.26$; age $4: r=$ $-.35)$ and across age (CU3 $\rightarrow$ prosociality4: $\mathrm{r}=-.31$; prosociality3 $\rightarrow$ CU4: $r=-.16)$. A biometric cross-lagged model revealed a cross-lagged effect from CU to prosocial only. This demonstrates that whereas high $\mathrm{CU}$ at age 3 results in lower prosociality at age 4, low prosociality is not putting the child at risk for high CU 1 year later. At both ages there were significant genetic, shared, and nonshared environmental influences on $\mathrm{CU}$, and genetic and nonshared environmental influences on prosociality. At age 3, the phenotypic relation between $\mathrm{CU}$ and prosociality was explained by overlap in genetic effects $\left(r_{\mathrm{g} 3}=-.56\right)$. At age 4 , there were novel genetic and nonshared environmental effects on $\mathrm{CU}$ and prosociality. The covariance at age 4 was due to shared genetic effects from age 3 , and novel nonshared environmental influences at age $4\left(\mathrm{r}_{\mathrm{e} 4}=-.32\right)$. Although genetic factors contributed to stability and reciprocal effects, they also demonstrated developmental change in both phenotypes.

\section{Twin analysis of the association between flow proneness and work-related depressive symptoms and burnout}

Ana Butkovic, University of Zagreb, Croatia; Miriam Mosing, Karolinska Institutet, Sweden; Fredrik Ullen, Karolinska Institutet, Sweden

Most behaviour genetics research on depression and burnout has focused on adverse causal factors. However, various factors related to positive coping and well-being in daily life have been shown to be negatively associated with depression and burnout. In the present study, using a large, genetically informative sample of Swedish twins, we aim to understand the genetic aetiology underlying the relationship between flow proneness and work-related depressive symptoms and emotional exhaustion, as a measure of burnout. The full sample consisted of 10,120 twins with a score for at least one of the studied variables, including 2,337 full twin pairs (1,114 MZ and 1,223 DZ pairs) and 5,446 single twins without the co-twin participating. Their age ranged between 27 and 54 years $(M=40.7, S D=7.75)$. Participants filled in the Swedish Flow Proneness Questionnaire, a six item subscale of the Hopkins Symptom Checklist (SCL) depression scale, focusing on work-related symptoms, and the Emotional Exhaustion subscale of the Maslach Burnout Inventory-General Survey. Higher scores indicated more flow proneness, less depressive symptoms and less burnout. Phenotypic correlations were 0.62 between depressive symptoms and burnout, 0.44 between depressive symptoms and flow proneness, and 0.35 between burnout and flow proneness. Broadsense heritabilities (G) were 33\% for flow proneness and emotional exhaustion, and $33 \%$ for males and $35 \%$ for females for depressive symptoms. A trivariate GE Cholesky decomposition was fitted, demonstrating significant genetic, as well as non-shared environmental, components of the associations between the three variables. In summary, genetic liability has a substantial influence on associations between flow and emotional problems at work (depression, 
burnout). However, the presence of significant environmental correlations would be in line with a causal relationship between flow and work related depression and burnout, which in turn may suggest that interventions which increase flow could potentially reduce emotional problems at work.

\section{Genetic, prenatal, postnatal, and endocrine influences on adolescent smoking}

Kristine Marceau, Purdue University, United States; Leslie Brick, Brown University, United States; Valerie Knopik, Brown University, United States; Menno Reijneveld, University Medical Center, Groningen, Netherlands

Multiple bio-behavioral mechanisms of the development of adolescent smoking have been identified, including genetic risk, prenatal adversity, blunted cortisol reactivity, less warm/more hostile parenting, and behavior problems within the broader developmental context. Each developmental influence highlighted here does not occur in a vacuum, and what is needed are studies that examine these multiple influences together to identify the best predictors and developmental pathways to substance use. The genetic underpinnings of cortisol functioning are relatively well documented, but few studies have tested whether genetic or environmental mechanisms (endogenous or exogenous) underlie cortisol-smoking associations, despite evidence that some of the same genes are associated with cortisol and smoking (in different studies). We tested whether a polygenic score of cortisolrelated genes, prenatal adversity, and parenting at age 11 years predicted cortisol reactivity to a social stress challenge at age 11 years, trajectories of internalizing and externalizing problems from age 1116 , and smoking frequency at 16 years using Genome-wide association and phenotypic data from 2230 Dutch adolescents in the TRacking Adolescents' Individual Lives Survey. We used a twofold (discovery/test) set approach to (1) create polygenic scores related to cortisol function and then (2) test the main effects and full structural equation models including genetic, prenatal, parenting, and behavioral trajectory predictors of smoking. Polygenic scores were constructed from genes empirically linked to cortisol functioning and/ or listed in one of several biological pathways key to cortisol production: 217 genes were identified, 1071 (non-imputed) singlenucleotide polymorphisms (SNPs) on 1354 individuals were included after standard quality control and pruning for linkage disequilibrium. Zero-order correlations revealed that no SNPs were significantly related to cortisol after adjustment for multiple testing. Results generally suggested that cortisol, prenatal adversity, and colder parenting all predicted smoking frequency, but that the polygenic score was unrelated to (a) cortisol, (b) other predictors, and (c) smoking frequency. Findings suggest that cortisol relates to smoking via behavioral, and not via genetic mechanisms.

\section{Familial factors associated with early versus late onset depression}

Kristy Cuthbert, Boston University, United States; Mark Logue, Boston Univ., United States; Ruth Mckenzie, Boston Univ., United States; Rosemary Toomey, Boston Univ., United States; Matt Panizzon, University of California, San Diego, United States; Carol Franz, University of California, San Diego, United States; Ming Tsuang, Univ. of California, San Diego, United States; William Kremen, University of California, San Diego, United States; Michael Lyons, Boston University, United States
According to the National Institute of Aging, depressive symptoms in older adults peak around age 50 and then again after age 80 . Research suggests that early onset depression (EOD) differs from late onset depression (LOD) in associated personality characteristics, childhood events, family psychiatric history, and neuroimaging characteristics. However, it is unclear whether observed differences represent genetically different manifestations of depression or the influence of environmental factors. This study examined 288 male twins from the Vietnam Era Twin Study of Aging who reported any lifetime depression to examine familial and genetic contributions to EOD and LOD. LOD was defined as onset occurring at 40 years of age and older. GEE analyses indicated that a history of alcohol misuse in father $(\mathrm{OR}=1.89, \mathrm{p}=.016)$, cotwin $(\mathrm{OR}=2.68, \mathrm{p}<.001)$, and closest sibling $(\mathrm{OR}=2.00, \mathrm{p}=.036)$ increased the odds of developing EOD relative to LOD. A history of depression in mother $(\mathrm{OR}=2.35$, $\mathrm{p}<.001)$, cotwin $(\mathrm{OR}=2.13, \mathrm{p}=.002)$, and closest sibling $(\mathrm{OR}=1.91$, $\mathrm{p}=.019$ ) increased the odds of developing EOD relative to LOD. A history in any family member of drug misuse or trouble with the law did not increase the odds of developing either EOD or LOD. Neither comparisons of concordance rates by zygosity or analyses of polygenic risk scores for depression provided clear-cut evidence for genetic influences on EOD versus LOD. However, given the relatively small number of individuals with depression, these methods were underpowered. Our results indicate that there are significant familial influences on whether an individual experiences EOD versus LOD. However, we were unable to distinguish family environmental influences from genetic influences responsible for this effect. There are two plausible reasons for the pattern we observed. First-degree relatives may share a genetic risk for depression and genetic influences on depression may also impart a risk for alcohol abuse, particularly in males. It is also plausible that the family environment in which a parent or sibling has depression or alcohol abuse may be depressogenic. It is likely that our results reflect both genetic and environmental factors.

\section{Birth weight as an independent predictor of general and specific mental health problems in adulthood: a population-based, within-sibling pair analysis}

Erik Pettersson, Karolinska Institutet, Sweden; Henrik Larsson, Karolinska Institutet, Sweden; Brian D’Onofrio, Indiana University, United States; Paul Lichtenstein, Karolinska Institutet, Sweden

Background: Low birth weight is associated with mental health problems in child- and adulthood. However, it remains unclear if birth weight influences general versus specific mental health problems, and if such associations remain after controlling unmeasured confounds shared within sibling pairs.

Aims: (1) Examine whether birth weight predicts general versus specific mental health problems. (2) Examine whether these associations remain after controlling unmeasured confounds shared within sibling pairs.

Method: We identified the oldest two siblings (differing by five years or less) in each family in Sweden born between 1958 and 1999 (N= 1,460,699 sibling pairs). Birth weight was retrieved from the Medical Birth Register. Psychiatric diagnoses (depression, anxiety, PTSD, OCD, eating disorder, alcohol abuse, drug use, autism, ADHD, bipolar, schizophrenia, and schizoaffective disorder) were retrieved from the National Patient Register (assigned by the attending physician in accord with ICD-8, 9, or 10).

Results: A four-factor EFA fit the data well (RMSEA=0.002; $\mathrm{CFI}=$ 0.992), which we rotated toward one general and four specific factors (internalizing, externalizing, neurodevelopmental, and psychotic problems). We then regressed the EFA on birth weight using regular 
and fixed effects regression. Birth weight was significantly associated with general $(\beta=-0.02, \mathrm{p}<.001)$, externalizing $(\beta=-0.04, \mathrm{p}<.001)$, and neurodevelopmental problems $(\beta=-0.03, \mathrm{p}<.001)$. These associations remained significant after controlling unmeasured confounds shared within sibling pairs.

Discussion: In this population-based, within-sibling comparison study, low birth weight predicted general, externalizing, and neurodevelopmental problems even after controlling unmeasured confounds shared within sibling pairs. Restricted fetal growth indicates that insufficient energy supply for organ development contributes to a wide range of mental health problems in adulthood.

\section{Returns to birth weight in an egalitarian setting. Can parents mitigate negative effects of low birth weight?}

Antonie Knigge, Utrecht University, Netherlands; Jornt Mandemakers, Utrecht University, Netherlands

Low birth weight as a marker of the prenatal environment has been identified as a predictor of long term health and development. This finding exemplifies research showing the long arm of early disadvantage. However, the negative effect of low birth weight could in part be due to genetic and family environmental confounders. Relatively few studies use studies of twins and/or siblings to control for genetic and family confounders. Moreover, it is not clear how persistent the effects of early disadvantage are: they could be stable or even increasing over the life course (cumulative disadvantage), or early disadvantage could be mitigated over time. We argue that this may depend on parental behavior: parents may differentiate investments between siblings (twins) in order to reinforce or compensate initial differences. We hypothesize that higher SES parents are better able to reduce the negative effects of low birth weight than lower SES parents. The frequent occurrence of low birthweight among twins makes this an important area to investigate. Furthermore, differential treatment of twins has important bearings for twin research. We examine the effect of low birth weight on cognitive ability, height, and health in a new representative sample of twins and siblings from Germany (Twinlife). Currently we use the released first half of the first wave, which contains information on about 2,000 same-sex twin pairs. The first wave comprises 4 ages $(5,12,17,24)$. Preliminary results indicate that effects of birth weight remain even in within-MZ estimates. Future work will use the full sample (4,000 pairs) and also address the questions:

Are the effects of early disadvantage on children's development and health stable, reinforced, or mitigated over the life course?

How do parents influence whether early disadvantages are reinforced or mitigated?

Do parents invest differently in their children depending on differences in birth weight within twin pairs?

Are initial differences between twins/siblings more often compensated for by more resourceful parents?

\section{Stressful life events and personality disorders in etiology of alcohol use disorder: a twin study using a novel biometric version of the statistical mediation model}

Tom Rosenström, Norwegian Institute of Public Health, Norway; Fartein Ask Torvik, Norwegian Institute of Public Health, Norway; Steven Aggen, Virginia Commonwealth University, United States; Nikolai Olavi Czajkowski, Norwegian Institute of Public Health,
Norway; Eivind Ystrom, Norwegian Institute of Public Health, Norway; Espen Eilertsen, Norwegian Institute of Public Health, Norway; Robert F. Krueger, University of Minnesota, United States; Nathan Gillespie, Virginia Commonwealth University, United States; Ted Reichborn-Kjennerud, Norwegian Institute of Public Health, Norway

Alcohol use disorder (AUD) is a common psychiatric disorder that induces a heavy burden on public health. It represents a failure of acceptable social behavior and of maintaining moderation. DSM-IV Antisocial and Borderline Personality Disorders (PDs) and associated conduct problems and impulsive behaviors are known risk factors of AUD. Existing evidence indicates that childhood stressful life events (SLEs) play a role in the development of all these disorders. While it may seem likely that PD traits mediate at least some of the effects of childhood SLEs on adulthood AUD, demonstrating causality has turned out difficult because of possible genetic confounding. The statistical mediation model assumes lack of such confounding, and is frequently used to assess hypotheses regarding causal chains of events. Here we extend the model to a biometric version that can both test the assumed lack of confounding and estimate the mediation effects. The extended model is then applied to interview and survey data on PDs, AUD, and SLEs on 2801 Norwegian Twins drawn from the Norwegian Institute of Health Twin Panel data. Our findings indicated that Antisocial PD and its precursor, childhood conduct disorder, mediated the (primarily shared environmental) effects of childhood SLEs on AUD, with no indication of confounding. The mediation path via Borderline PD was confounded, whereas self-harming impulsivity (a component of Borderline PD) again mediated SLE effects on AUD. The total phenotypic effect of SLE count on AUD was $\sim 0.24$ standard deviations in AUD liability $(\mathrm{CI}=0.11-0.37)$, more than half of which was mediated by the PD traits. In conclusion, development of antisocial and impulsive traits may explain a large part of the AUD risk related to childhood adversity, whereas the role of borderline traits in this etiologic pathway remains unclear.

\section{Towards incorporating genetic risk scores into network models}

\section{Adela-Maria Isvoranu, University of Amsterdam, Netherlands}

In the past decade, network models in the fields of psychopathology and psychiatry have gained considerable attention and recognition. In such network models, mental disorders are no longer regarded as reflective latent variables, but are assumed to arise from interactions between symptoms and other psychological, biological, and sociological agents. Several studies have recently focused on disentangling relations between environmental risk factors and individual symptoms of specific mental disorders, but in spite of the clear potential clinical relevance, no studies to date investigated the impact of genes on symptom networks. This talk will therefore introduce a theoretical framework on how genetic risk scores could be incorporated into network models, followed by an empirical example of a symptom network of psychosis.

\section{Gene $\times$ SES interaction on child achievement and cognition in a U.S. sample}

Susan Gross, Case Western Reserve University, United States; Carol Gross, Case Western Reserve University, United States; Lee Thompson, Case Western Reserve University, United States; Stephen Petrill, The Ohio State University, United States 
The prediction that children raised in higher socioeconomic status homes will experience higher heritability of cognitive traits than children raised in lower socioeconomic status homes has been supported in samples collected in the United States (Tucker-Drob \& Bates, 2014). The present study seeks to explore the effects of the gene $\times$ SES interaction in a sample of twins collected from the Western Reserve Reading and Math Project. In total, 754 twins (MZ: $\mathrm{n}=294$; DZ: $\mathrm{n}=460$ ) living in the midwestern U.S. were assessed on SES at both the family and school level. Nine waves of data, including IQ, reading achievement, and math achievement at various time points, were collected via in-person testing, from ages 6 to 15 . The gene $\times$ SES interaction was explored using a set of models with main effects of SES in combination with moderation of A, C, and/or E (Purcell, 2002). Stability and change was also assessed across the school-years. Results from gene $\times$ SES modeling with SES characterized by both family and school level will be discussed.

\section{References}

Purcell S (2002) Variance components models for gene-environment interaction in twin analysis. Twin Research 5:554-571.

Tucker-Drob E. M., Bates T. C. (2016). Large cross-national differences in gene $\times$ socioeconomic status interaction on intelligence. Psychological Science, 27, 138-149. doi:10.1177/0956797615612727

\section{Genetic and environmental influences on adolescent addition problem strategies}

Carol Gross, Case Western Reserve University, United States; Susan Gross, Case Western Reserve University, United States; Lee Thompson, Case Western Reserve University, United States; Stephen Petrill, The Ohio State University, United States

Strategies used to solve addition problems without pencil and paper have been evaluated and connected to math achievement in multiple studies of children and adults. However, only a few studies have used adolescent samples, and addition strategies have not been evaluated in a behavior genetic framework. In this study we evaluated the addition strategies used by a group of adolescent twins ( $77 \mathrm{MZ}$ pairs and 136 DZ pairs) from the Western Reserve Reading and Math Project. Participants solved 20 addition problems (14 simple and 6 complex) and reported the strategies that they used to solve each problem. Strategies included retrieval, decomposition, and counting. The genetic and environmental influences on which strategies were used were calculated for simple, complex, and all addition problems. Strategy use measures for complex problems had a significant estimate of heritability. All measures of strategy use had significant nonshared environmental influences, and none of the measures had significant shared environmental influences.

\section{Mapping geographical variation in the genetic and environmental aetiology of autism spectrum disorder and attention deficit hyperactivity disorder in Sweden}

Zoe Reed, University of Bristol, United Kingdom; Henrik Larsson, Karolinska Institutet, Sweden; Paul Lichtenstein, Karolinska Institutet, Sweden; Oliver Davis, University of Bristol, United Kingdom
Observational studies suggest that the prevalence of autism spectrum disorder (ASD) risk varies geographically between urban and rural areas, with increased urbanicity associated with greater risk (1). In contrast, attention deficit hyperactivity disorder (ADHD) has shown less evidence of urban-rural prevalence differences (1). We applied our spACE spatial analysis approach (2) to explore whether the genetic and environmental aetiology of these disorders varies in a similar way, using data from 8,612 twin pairs from the Swedish Twin Registry (3). Information on ASD and ADHD was collected using the A-TAC questionnaire administered via telephone interview with parents when the twins were either 9 or 12 years old. Twin pairs were matched to Small Area Marketing Statistics (SAMS) locations by Statistics Sweden and we assigned the twin pairs to the centroids of these areas. By running a series of twin structural equation models in OpenMx while weighting the contribution of the twin pairs by the inverse of their distance from the point of estimation, we were able to produce maps of the variation in the genetic and environmental influences on ASD and ADHD. We will present these maps, and discuss their implications for our understanding of the aetiology of these disorders.

\section{References}

1. Vassos, E., Agerbo, E., Mors, O. \& Pedersen, C. B. Urban-rural differences in incidence rates of psychiatric disorders in Denmark. $\mathrm{Br}$ J Psychiatry 208, 435-440 (2016).

2. Davis, O. S. P., Haworth, C. M. A., Lewis, C. M. \& Plomin, R. Visual analysis of geocoded twin data puts nature and nurture on the map. Mol Psychiatr 17, 867-874 (2012).

3. Magnusson, P.K.E. et al. The Swedish Twin Registry: establishment of a biobank and other recent developments. Twin Res and Hum Genet, 16, 317-329 (2012).

\section{Can genetic variants underlying subjective well-being predict age-related health problems?}

Miriam Mosing, Karolinska Institutet, Sweden; Nancy L. Pedersen, Karolinska Institutet, Sweden; The Igems Consortium, Sweden

In a time where population aging is one of the most important demographic phenomena, the idea of predicting future health has received great attention with the hope of developing intervention strategies. Interestingly, simple measures of subjective well-being (SWB) based on a single item, are often superior to more objective clinical assessment for predicting an individuals' morbidity and mortality, suggesting that SWB is a powerful predictor of future health (Idler \& Benyamini, 1997). However, still little is known about the genetic architecture underlying this link between self-perceived measures of well-being and healthy aging. Recently, we identified genetic polymorphisms underlying SWB (rs3756290, rs2075677, and rs4958581) using genome-wide data of more than 298,000 individuals (Okbay et al., 2016). Here we derived polygenic risk-scores based on the genome-wide association results of SWB to predict age-related health problems as measured by the Cumulative Illness Rating Scale (CIRS). CIRS is a cumulative score based on medical problems in 13 different organ systems which reflects common problems of the elderly. One or more existing diseases per organ system will result in a score of one for that particular system, with a final score between zero (disease free) to thirteen. CIRS scores as well as genetic information were available for 8,000 individuals from the IGEMS consortium. The polygenic risk-score was regressed on the observed CIRS scores to assess the degree to which variability in CIRS is explained by variability in genes underlying SWB. Findings and implications will be discussed. 


\section{References}

Idler, E.L., Benyamini, Y. (1997). Self-rated health and mortality: a review of twenty-seven community studies. J Health Soc Behav, 38 (1), 21-37.

Okbay, A., Baselmans, B.M., De Neve, J.E., Turley, P., Nivard, M.G., Fontana, M.A., ... Cesarini, D. (2016). Genetic variants associated with subjective well-being, depressive symptoms, and neuroticism identified through genome-wide analyses. Nat Genet, 48 (6), 624-633.

\section{Triplets, birthweight and left-handedness}

Kauko Heikkilä, University of Helsinki, Finland; Toos Van Beijsterveldt, Vrije Universiteit Amsterdam, Netherlands; Jari Haukka, University of Helsinki, Finland; Matti Iivanainen, University of Helsinki, Finland; Aulikki Saari-kemppainen, Helsinki University Hospital, Finland; Dorret Boomsma, Vrije Universiteit Amsterdam, Netherlands; Yoshie Yokoyama, Osaka City University/Dept Community Hlth Nursing, Japan; Eero Vuoksimaa, University of Helsinki, Finland

Introduction: Handedness is reported to originate early in pregnancy, but mechanisms of development are not well known. Understanding associations of laterality could potentially help to understand brain development better. Earlier studies have indicated that very-lowbirthweight $(<1500 \mathrm{~g})$ or extremely-low-birthweight $(<1000 \mathrm{~g})$ singletons have higher prevalence of left-handedness than children of normal birthweight. Birthweight is naturally smaller in multiples with twins and triplets being about 1 and $1.5 \mathrm{~kg}$ smaller than singletons, respectively. Studies have indeed indicated higher prevalence of lefthandedness in multiples compared to singletons. However, it is not known if birthweight is related to handedness in triplets.

Methods: We studied birthweight and handedness in two large samples of triplets from Japan ( $\mathrm{N}=1305$ individuals) and Netherlands ( $\mathrm{N}$ $=892$ individuals). We used logistic regression to test if birthweight was related to handedness (left versus right excluding ambidextrous participants). Clustered data (triplets within families) was taken into account in all analyses. Motor development of the children was tested for associations with handedness and birthweight.

Results: Left-handers had (Japan $\mathrm{M}=1599 \mathrm{~g}$; The Netherlands $\mathrm{M}=$ $1794 \mathrm{~g}$ ) significantly ( $\mathrm{ps}<.01)$ smaller birth weight compared to right-handers (Japan M=1727 g; The Netherlands $M=1903 \mathrm{~g}$ ).

The association between birth weight and handedness remained significant in both data sets when birth order, sex and infertility treatment were in the same model. Among Japanese triplets the association also remained when adjusting by gestational age. In Dutch triplets both birthweight and gestational age had a significant association with handedness. However, when these two were simultaneously in, the two associations became nonsignificant. In accordance with earlier twin studies zygosity did not have an association with handedness. Left-handedness was related to delayed motor development but this association was not evident when adjusting for birth weight.

Conclusions: We showed that lower birthweight is associated with higher prevalence of left-handedness in triplets. Because of two datasets the result could be replicated. Left-handers had delayed motor development in comparison to right-handers, but the difference vanished when adjusted by birth weight. Our results stress the importance of multiples and birthweight in handedness studies.
Alzheimer's disease polygenic risk score identifies mild cognitive impairment in adults in their $50 \mathrm{~s}$

William Kremen, University of California, San Diego, United States; Mark Logue Boston Univ., United States; Matthew Panizzon, University of California, San Diego, United States; Jeremy Elman, University of California, San Diego, United States; Nathan Gillespie, Virginia Commonwealth University, United States; Sean Hatton, University of California, San Diego, United States; Daniel Gustavson, University of California, San Diego, United States; Ole Andreassen, University of Oslo/NORMENT, Norway; Anders Dale, University of California, San Diego, United States; Michael Lyons, Boston University, United States; Michael Neale, Virginia Commonwealth University, United States; Chandra Reynolds, University of California, Riverside, United States; Carol Franz, University of California, San Diego, United States

Because the pathological process in Alzheimer's disease (AD) begins decades before onset of dementia, early identification of risk is paramount. We asked whether an AD polygenic risk score (PRS) derived from IGAP data could identify mild cognitive impairment (MCI)-a transitional phase in the progression from normal cognition to AD-in adults who were only in their $50 \mathrm{~s}$. Participants were 1176 white, nonHispanic community-dwelling men of European ancestry (mean age $=$ 56; $89 \%<60)$ : $\mathrm{N}=1050$ cognitively normal $(\mathrm{CN}) ; 83$ amnestic MCI (aMCI); 43 non-amnestic MCI (naMCI). Diagnosis was based on the Jak-Bondi actuarial/neuropsychological approach. We controlled for non-independence of twins, the first 3 principal components from the SNP data, and factors affecting cognitive function: age; depression; hypertension; diabetes; head injury. We tested $6 \mathrm{P}$-value thresholds (0.05-0.50) for SNPs included in the PRS. After correction for multiple testing, higher PRSs were associated with significantly greater odds of being aMCI than $\mathrm{CN}$ (odds ratios [ORs] $=1.36-1.43$ for thresholds $\mathrm{P}<.20-0.50$ ). For the most significant threshold, the $\mathrm{OR}=3.22$ for the upper versus lower quartile of the PRS distribution. ORs remained significant after excluding APOE-related SNPs from the PRS. Diabetes was associated with significantly increased odds of having naMCI (ORs $=3.10-3.41$ for thresholds $\mathrm{P}<.05-0.50)$. With a realistic aMCI prevalence of $7 \%$, prediction accuracy for $\mathrm{CN}$ versus aMCI was high (AUCs $=0.981-0.995$ ). With cut-offs resulting in high negative and positive predictive values (NPV, PPV), specificity was high and sensitivity was moderate. This threshold may be useful for clinical trials, providing greater certainty that cases are truly cases by substantially reducing false positives. With cut-offs resulting in high sensitivity and specificity, NPV was high and PPV was moderate. This threshold may be useful for screening individuals to be referred for further evaluation because people classified as controls would be highly likely to be true controls, whereas aMCI would include increased false positives. Results are consistent with aMCI being more AD-related and naMCI having more vascular/inflammation components. The results support our neuropsychologically-defined basis for diagnosing MCI, and the use of PRSs in clinical trials aimed at early intervention. Such efforts are likely to be key factors for more effectively treating or slowing the progression of $\mathrm{AD}$.

\section{Risk preference shares the psychometric structure of major psychological traits}

Renato Frey, University of Basel, Switzerland; Andreas Pedroni, University of Zurich, Switzerland; Rui Mata, University of Basel, Switzerland; Jörg Rieskamp, University of Basel, Switzerland; Ralph Hertwig, Max Planck Institute for Human Development, Berlin, Germany 
To what extent is there a general factor of risk preference, $\mathrm{R}$-akin to $\mathrm{g}$, the general factor of intelligence-that may complement domainspecific dimensions? And can risk preference be regarded a stable psychological trait? These conceptual issues persist because few attempts have been made to integrate multiple risk-taking measures, in particular measures from different and largely unrelated measurement traditions (self-reported propensity measures assessing stated preferences; incentivized behavioral measures eliciting revealed preferences; frequency measures assessing concrete risky activities). We provide a substantive empirical foundation for addressing these issues with a comprehensive psychometric approach (1,507 healthy adults completing 39 risk-taking measures), and found that correlations between propensity and behavioral measures were weak. Yet, a general factor of risk preference, R, emerged from stated preferences, and generalized to frequency measures of concrete risky activities. Moreover, $\mathrm{R}$ proved highly stable across time, indicative of a psychological trait. Our findings offer a first step towards a general theory of risk preference and have implications for its assessment.

\section{Chewing habits and EGFR overexpression in oral squamous cell carcinoma patients of Pakistan}

Yumna Mirza, Aga Khan University Hospital, Pakistan; S. M. Adnan Ali, Aga Khan University Hospital, Pakistan; Muhammad Sohail, Aga Khan University Hospital, Pakistan

No abstract available

\section{Age and sex moderation of genetic and environmental influences on a harmonized measure of financial strain: IGEMS}

Deborah Finkel, Indiana University Southeast, United States; Chandra Reynolds, University of California, Riverside, United States; Catalina Zavala, University of Southern California, United States; Igems Consortium, University of Southern California, United States

One of the primary challenges facing groups that aim to combine data across existing twin studies is the harmonization of variables. Standard meta-analysis of effect sizes does not capture variability created by gene-environment interplay unless it is specifically modeled in the individual studies. To represent these individual differences, pooling data via harmonization is often required. Nine of the 15 twin studies of adult development and aging that are part of the IGEMS consortium included items assessing perceived financial strain, representing 10,756 individuals. Four different items assessed perceptions of financial strain: extent to which money covers needs (8 studies with 8769 participants), difficulty in paying monthly bills (5 studies with 5876 participants), economic situation compared to others (4 studies with 3398 participants), and whether there is money for extras (2 studies with 2986 participants). A factor model was used to create a harmonized measure of financial strain across studies and items: the best fitting model equated loadings across studies for similar items. The sample was 55\% women, included $3185 \mathrm{MZ}$ twins and $5228 \mathrm{DZ}$ twins, and age ranged from 24 to 98 . Twin analysis of genetic and environmental variance incorporating age as a continuous moderator and sex as a dichotomous moderator indicated both age and sex differences in the etiology of the harmonized financial strain score. As predicted by the diathesis-stress model, increased financial strain resulted in increased genetic variance in subjective health for women. In contrast, increased financial strain was associated with increased shared environmental variance for men, likely reflecting increasing salience of shared cultural conceptions of "good health".
Investigation of genetic factors underlying specific psychotic experience traits during adolescence and their relationship with psychiatric disorders

Angelica Ronald, Birkbeck College, University of London, United Kingdom; Oliver Pain, Birkbeck College/London School of Hygiene and Tropical Medicine, United Kingdom; Alastair Cardno, Leeds, United Kingdom; Daniel Freeman, University of Oxford, United Kingdom; Lu Yi, Karolinska Institutet, Sweden; Sebastian Lundström, University of Gothenburg, Sweden; Frank Dudbridge, Leicester University, United Kingdom; Paul Lichtenstein, Karolinska Institutet, Sweden

Psychotic experiences (PEs) are traits in the general population that at the extreme characterize the symptoms of disorders such as schizophrenia. It is common to have PEs during adolescence and they predict later psychiatric disorders (McGrath et al., 2016, Am J Psych). At the same time, the majority of PEs dissipate over time. Twin studies show that approximately $30-50 \%$ of the variance in PEs in mid-adolescence is explained by twin heritability (Zavos et al., 2014, JAMA Psychiatry). This study aimed to identify novel genetic variants associated with specific PE domains in adolescence, and to test for overlap in genetic influences between PEs as traits in adolescence and schizophrenia, bipolar disorder, and major depression in adults.

The full spectra of PEs, both in terms of severity and type (positive, cognitive, and negative), were assessed using self- and parentratings of quantitative traits in three European community samples aged 15-19 years (Final N including siblings=6297-10,098). A mega-genome-wide association study (mega-GWAS) was conducted on normalized scales with imputed data on a common reference panel across all samples. Several approaches (LD score regression, and polygenic risk score [PRS] analysis and AVENGME) were applied to estimate the degree of genetic covariance between PEs in adolescence and clinically recognized psychiatric conditions (schizophrenia, bipolar disorder, and major depression).

Genomic-relatedness-based restricted maximum likelihood returned SNP-heritability estimates of 3-9\%. In the largest GWAS on PEs to date, mega-analysis returned one genome-wide significant association with an imputed SNP. PRS analysis revealed that the Psychiatric Genomic Consortium 2 (PGC2) schizophrenia PRS significantly predicted all domains of adolescent PEs (Paranoia and Hallucinations only in non-zero scorers), explaining $0.1-0.2 \%$ of variance. The PGC2 major depression PRS significantly predicted Anhedonia and Parent-rated Negative Symptoms in adolescence, explaining $0.1-0.2 \%$ of variance. Other methods concurred in showing modest genetic covariance between specific PEs and psychiatric disorders.

Psychotic experiences in the community during adolescence show additive genetic effects and partly share genetic influences with psychiatric disorders, specifically schizophrenia and major depression.

\section{Genetic influences on parenting are partially explained by parental internalizing symptoms}

Amanda Broderick, The Pennsylvania State University, India; Amanda Griffin, The Pennsylvania State University, United States; Amanda Ramos, The Pennsylvania State University, United States; David Reiss, Yale University, United States; Jody Ganiban, The George Washington University, United States; Erica Spotts, National Institutes of Health, United States; Paul Lichtenstein, Karolinska Institutet, Sweden; Jenae Neiderhiser, The Pennsylvania State University, United States 
Parents who experience higher levels of depression and anxiety symptoms tend to engage in more negative parenting (high conflict and harshness). Both internalizing symptoms and parenting are genetically influenced, and prior studies have shown that parent characteristics such as anxious personality traits share genetic underpinnings with negative parenting. The present study uses a sample of twin parents of adolescents (Twin and Offspring Study in Sweden: TOSS) to assess the contributions of genetic, shared environmental, and non-shared environmental influences on the overlap between parental internalizing symptoms and parenting behavior. TOSS is comprised of 909 pairs of same-sex twin parents of an adolescent $(\mathrm{n}=386 \mathrm{MZ}$ pairs, $63 \%$ female, Mage $=44.89$ years, $\mathrm{SD}=$ 4.89). Adolescent cousin pairs were same-sex and within 4 years of age of one another (Mage $=15.75$ years, $\mathrm{SD}=2.4049 \%$ female). Twins reported on their own internalizing symptoms using the Center for Epidemiological Studies Depression scale and the psychic and somatic anxiety subscales of the Karolinska Scales of Personality. Twin parents also reported on their harsh or conflictual parenting using the Child Rearing Issues and Expressed Emotion measures. Composite scores were created for parent-rated internalizing and negative parenting and were significantly correlated $(r /=0.31$, $\mathrm{p}<.001$ ). A correlated bivariate factor model indicated that parental internalizing symptoms and negative parenting were explained by genetic and nonshared environmental influences $(\mathrm{A}=51 \% \mathrm{E}=49 \%$; $\mathrm{A}$ $=34 \% \mathrm{E}=64 \%$, respectively) with a significant genetic $\left(\mathrm{r}_{\mathrm{g}}=.44\right.$, $\mathrm{p}<.01)$ and nonshared environmental $\left(\mathrm{r}_{\mathrm{e}}=.24, \mathrm{p}<.01\right)$ correlation between the two constructs. Shared environmental influences did not contribute to either parental internalizing symptoms or to negative parenting, thus there was with no significant contribution of shared environmental influences to the covariance of the two. Additional analyses found no evidence of differences between mothers and fathers.

\section{The association between disruptive behaviors and self- harm/suicide attempt: an adolescent twin study}

Lauren O'Reilly, Psychological \& Brain Sciences, Indiana University, United States; Patrick Quinn, Psychological \& Brain Sciences, Indiana University, United States; Sebastian Lundström, University of Gothenburg, Sweden; Henrik Larsson, Karolinska Institutet, Sweden; Paul Lichtenstein, Karolinska Institutet, Sweden; Brian D'Onofrio, Indiana University, United States.

Disruptive behaviors (i.e., symptoms of conduct and oppositional defiant disorder) are a risk factor for suicidal behavior, although few behavior genetics studies have investigated this association. This study aimed to examine: (1) the genetic and environmental contributions to adolescent suicide attempt/self-harm (SA/SH) and (2) the association between childhood disruptive behaviors childhood and adolescent SA/SH. We analyzed data from the Child and Adolescent Twin Study of Sweden, a population-based study of twins (i.e., 29,000 twins at age 9 and 9,000 twins through age 18) born in Sweden from 1992 to 2007. When twins were 9, their parents completed the Autism-Tics, AD/HD and other Comorbidities Inventory, which indexed oppositional/conduct symptoms. When twins $w_{\mathrm{r}} \mathrm{e} 18$, they completed the Lifetime History of Aggression questionnaire and the Brief Obsessive-Compulsive Survey, which indexed self-harm and suicide attempts. We also included inpatient/outpatient suicide attempts via ICD-10 codes from the National Patient Register. We conducted a confirmatory factor analysis of four SA/SH items. All items loaded onto a single factor, which was moderately heritable (hsup $2 / \sup =0.68,95 \%$ CI [0.21-1.14]) with the remaining variance attributable to nonshared environmental factors. We also conducted population-wide and within-twin-pair logistic regression models to assess the association between disruptive behaviors and $\mathrm{SA} / \mathrm{SH}$. Every one increase in disruptive behaviors symptoms was associated with $\mathrm{SA} / \mathrm{SH}$ in the population $(\mathrm{OR}=1.06$ [1.03-1.09]). The magnitude of the association remained in within-twin-pair analyses $(\mathrm{OR}=1.08$ [0.99-1.19]), and there were no differences between identical and fraternal twins. We found comparable results using diagnostic cutoffs. This study demonstrated two major findings: (1) the self-harm and suicide attempt items loaded onto a single factor, which was moderately heritable, and (2) genetic and shared environmental factors did not appear to confound the association between disruptive behaviors and SA/SH. This is the first study to examine the etiology of adolescent suicidality using behavior genetics.

\section{MTAG: multi-trait analysis of GWAS implicates novel loci for depressive symptoms, neuroticism, and subjective well-being}

Raymond Walters, Broad, United States; Patrick Turley, Massachusetts General Hospital; Broad Institute of MIT and Harvard, United States; Omeed Maghzian, Harvard University, United States; Aysu Okbay, Vrije Universiteit Amsterdam, Netherlands; James Lee, University of Minnesota, Twin Cities, United States; Mark Fontana, University of Southern California, United States; Tuan Anh NguyenViet, University of Southern California, United States; Nicholas Furlotte, 23andMe, United States; 23andme Research Team, 23andMe, United States; Social Science Genetic Association Consortium, United States; Patrik K. E. Magnusson, Karolinska Institutet, Sweden; Sven Oskarsson, Uppsala University, Sweden; Magnus Johannesson, Stockholm School of Economics, Sweden; Peter Visscher, The University of Queensland, Australia; David Laibson, Harvard University, United States; David Cesarini, National Bureau of Economic Research; New York University; Institutet för Näringslivsforskning, United States; Benjamin Neale, Broad, United States; Daniel Benjamin, University of Southern California, United States

The standard approach in genome-wide association studies (GWAS) is to meta-analyze association statistics from cohort-level GWAS of a single trait. Such single-trait analyses do not exploit information that may be available from GWAS of other correlated traits. We introduce a method, Multi-Trait Analysis of GWAS (MTAG), which jointly analyzes GWAS results for several related traits, thereby boosting statistical power to detect genetic associations for each trait. ${ }^{1}$ MTAG is a generalization of standard, inverse-variance-weighted metaanalysis. The estimator takes summary statistics from at least two single-trait GWASs and, after jointly analyzing these, outputs traitspecific association statistics. The set of results for each trait represents an optimal combination of the information from the single-trait summary statistics, accounting for sample overlap and correlated genetic effects between the GWAS. The resulting $p$ values can be interpreted like GWAS p values and used in the usual ways, for example, to prioritize SNPs for subsequent analyses including biological annotation. We demonstrate MTAG using data on depressive symptoms (DEP; effective $\mathrm{N}=354,862$ ), neuroticism (NEUR; $\mathrm{N}=$ $168,105)$, and subjective well-being (SWB; $N=388,538$ ). Comparing single-trait GWAS to MTAG, the number of genome-wide significant loci increases from 32 to 74 for DEP, from 9 to 66 for NEUR, and from 13 to 60 for SWB. These gains in statistical power from MTAG analyses are equivalent to increasing the sample size of the original, single-trait GWASs by $37 \%$ (DEP), $96 \%$ (NEUR), and $85 \%$ (SWB). The association statistics from MTAG are replicated in a large, wellphenotyped cohort ( $\mathrm{N}=6,857-8,307$ for the three phenotypes) and improve prediction accuracy of polygenic risk scores by approximately $25 \%$, consistent with theoretical calculations. Moreover, the 
MTAG results yield more informative bioinformatics analyses, suggesting a role of glutamatergic neurotransmission in depression.

\section{References}

1. Turley, P. et al. MTAG: Multi-Trait Analysis of GWAS. bioRxiv (2017).

\section{Tobacco dependence transition speed \& the power of quasi-causality}

Spencer Huggett, University of Colorado Boulder, United States; Alexander Hatoum, University of Colorado Boulder, United States; Robin Corley, University of Colorado Boulder, United States; Michael Stallings, University of Colorado Boulder, United States

Tobacco use is responsible for millions of deaths worldwide and is a tremendous financial burden for society. Increased understanding of the temporal development of tobacco use may indicate a more precise time window for targeted intervention and prevention. Early tobacco initiation increases risk for the progression to tobacco dependence, but is associated with substantially slower transitions to dependence. Using a co-twin control (CTC) design and a sample of $\sim 2800$ twins we investigated the quasi-causal relationship between age of tobacco initiation and transition times to dependence. One limitation of the CTC design is limited power to detect potentially causal relationships and little is known regarding the most powerful technique to model these associations in twins. Therefore, we conducted a power analysis manipulating sample size and the level of quasi-causal effect on different types of CTC designs. Using the most powerful CTC method from our power analysis, we analyzed the quasi-causal effect of early initiation with slower transitions to dependence and investigated potential confounding of zygosity. Our results elucidate the relationship between early tobacco use and slower transitions to dependence and may have important implications for discordant twin research and substance use prevention programs.

\section{Genetic and environmental influences on associations among parenting and child negative emotionality}

Elizabeth Shewark, test, United States; Amanda Ramos, The Pennsylvania State University, United States; Jody Ganiban, The George Washington University, United States; Daniel Shaw, University of Pittsburgh, United States; David Reiss, Yale University, United States; Misaki Natsuaki, University of California, Riverside, United States; Leslie Leve, University of Oregon, United States; Jenae Neiderhiser, The Pennsylvania State University, United States

Parents' responses to their children influences children's emotion development, however few studies have examined how children may influence how their parents respond to them. Using the Early Growth and Development Study $(\mathrm{N}=561)$, we examined how birth mother (BM) personality factors (negative affect versus control, orienting sensitivity, and agreeableness) influenced child negative emotionality (Child Behavior Questionnaire) at 4.5 years, and the subsequent associations with hostile or warm (Iowa Family Interaction Rating Scales) reactions from their adoptive parents (adoptive mother (AM) and father (AF)) at child age 6 and on later child behavior problems at child age 7 (Child Behavior Checklist). Several key indirect associations are noteworthy. High levels of BM control $(\beta=-.20)$ and orienting sensitivity $(\beta=-.13)$ evoked less warmth from AM at child age 6 which in turn was associated with more child externalizing problems a year later $(\beta=-.17)$. Child sadness at 4.5 years evoked less warmth from AM at 6 years $(\beta=-.15)$, which was in turn associated with more child externalizing problems at 7 years $(\beta=-.17)$. For the hostility models, child anger at child age 4.5 years evoked more hostility from both adoptive parents at child age 6 (AM: $\beta=.15$; AF: $\beta=.28$ ), which was associated with more child externalizing (AM: $\beta$ $=.28$; AF: $\beta=.29$ ) and internalizing problems (AF: $\beta=.25$ ). Higher levels of $\mathrm{BM}$ negative affect evoked more $\mathrm{AF}$ hostility $(\beta=-.13)$, which was associated with more child internalizing $(\beta=.25)$ and externalizing problems $(\beta=.29)$ a year later. These findings support prior research demonstrating the evocative role of children in parentchild interactions, and provide evidence that a portion of this association is heritable. The results also show that different types of negative emotions in children elicit different responses from parents.

\section{Genome-wide profiling of DNA methylome and transcriptome in peripheral blood monocytes for major depression: a monozygotic discordant twin study}

Jinying Zhao, University of Florida, United States; Yun Zhu, University of Florida, United States; Eric Strachan, University of Washington, United States; Emily Fowler, University of Washington, United States; Tamara Bacus, University of Washington, United States; Peter Roy-Byrne, University of Washington, United States

Major depressive disorder (MDD) is one of the most prevalent worldwide psychiatric disorders. Epigenetic mechanisms are thought to be involved in MDD, but the specific genes or genomic regions associated with MDD remain largely unknown. Here we conducted an epigenome-wide association study (EWAS) using the Illumina HumanMethylation450 BeadChip in peripheral blood monocytes from 47 monozygotic (MZ) twin pairs discordant for MDD. We identified 406 probes that were differentially methylated between depressed twins and their non-depressed co-twins. Region-based analysis identified 16 differentially methylated regions (DMRs) significantly associated with MDD at the level of FDR-adjusted $\mathrm{P}<.05$. These putative DMRs harbor candidate genes previously reported to be associated with MDD, and also several new loci containing genes involved in synaptic transmission or neurological functions. To determine the functional importance of DNA methylation alteration in gene regulation, we also performed genome-wide transcriptome analysis in blood monocytes of the same twins. Integrated analysis revealed both negative and positive correlations between DNA methylation and gene expression. A systems biology approach that combines gene co-methylation network with functional enrichment analysis elucidated distinct modules enriched with genes involved in neurotransmission or brain functions. Together, our results confirmed the role of DNA methylation in depression. Moreover, our study demonstrated that a well-designed monozygotic discordant co-twin control design could aid the discovery of novel DNA methylation signals, even in a limited sample size.

\section{Alcohol outlet density and alcohol use and problems: evidence for gene-environment interaction?}

Wendy Slutske, University of Missouri, United States; Arielle Deutsch, Pennsylvania State University, United States

The most widely-discussed contributor to the "alcohol environment" is availability, and one of the key contributors to availability is the 
density of alcohol outlets in one's community. However, studies of alcohol involvement that have incorporated gene-environment interactions have tended to emphasize the social and demographic characteristics of individuals or families rather than characteristics of the community- or neighborhood-level alcohol environment. The goal of this study was to examine whether living in a community with more alcohol outlets would facilitate the expression of the genetic propensity to drink and to develop alcohol-related problems in a genetically-informed national survey of United States young adults. The participants were 1,751 18-26 year-old twin, full-, and halfsibling pairs from Wave III of the National Longitudinal Study of Adolescent to Adult Health. Participants completed in-home interviews in which alcohol use and problems were assessed. Alcohol outlet densities were extracted from state-level liquor license databases, aggregated at the census tract level, and divided by the total land area for each census tract to derive the density of outlets. We examined whether estimates of genetic and environmental influences on alcohol use and problems varied as a function of the density of alcohol outlets in the community. Because the distribution of outlet densities across the United States is extremely skewed and they may only exert an effect at high levels, we dichotomized outlet densities at above (mean of 29 outlets per square kilometer) and below (mean of 1.5 outlets) the top decile. There was evidence to suggest that alcohol outlet density moderated the genetic diathesis to develop alcoholrelated problems; the age- and sex-adjusted heritabilities were 67 and $16 \%$ in high- and low-outlet-density communities, respectively. The results for other alcohol outcomes and for different types of alcohol outlets will be presented and limitations of this study will be discussed.

\section{Overlap and distinction between hedonic and eudaimonic well-being: a genetically-informative approach to elucidate current discussions}

Meike Bartels, Vrije Universiteit Amsterdam, Netherlands; Margo Van De Weijer, Vrije Universiteit Amsterdam, Netherlands; Gonneke Willemsen, Vrije Universiteit Amsterdam, Netherlands; Bart Baselmans, Vrije Universiteit Amsterdam, Netherlands

What well-being consists of has long been a question of great interest in a wide range of fields, dating back to ancient philosophy. How we define well-being has a large influence on clinical applications as well as public policy and social norms. It is therefore important to know whether the complexity of the manifold concept well-being can be captured in one framework. While a variety of definitions of the term well-being have been suggested, we assess the validity of a single framework for well-being. Previous research has shown that affective and cognitive measures of well-being, such as subjective happiness and satisfaction with life, load on one common genetic factors (Bartels and Boomsma, 2009). Much less is known, however, about the overlap between these hedonic measures of well-being and eudaimonic measures, such as flourishing and meaning to life.

With a molecular genetic approach, we investigated the overlap and distinction between hedonic (subjective) and eudaimonic (psychological) well-being. Using the large database of the Netherlands Twin Register, we will estimate genetic correlations. Furthermore, polygenic scores for the Well-being spectrum (including subjective well-being, depressive symptoms, and neuroticism) will be calculated based on the recent multivariate GWAMA (Baselmans et al., Biorxiv). With these PGS we will predict flourishing, one of the typical measures of Eudaimonic well-being.

\section{A longitudinal behavior genetic analysis of inhibitory control and ADHD symptoms from toddlerhood through early adolescence}

Jeffrey Gagne, University of Texas at Arlington, United States; Carol Van Hulle, University of Wisconsin, Madison, United States; Kathryn Lemery-Chalfant, Arizona State University, United States; Hill Goldsmith, University of Wisconsin, Madison, United States

Inhibitory control (IC) is a dimension of temperament and an executive function involving the ability to appropriately regulate behavior. In middle childhood, IC is negatively related to non-clinical behavior problems and Attention Deficit Hyperactivity Disorder (ADHD). Multiple twin studies indicate that IC is genetically influenced, however findings depend somewhat on the age of the participants and the assessment methodology (Gagne \& Saudino, 2010; 2016; Gagne \& Goldsmith, 2011). Parent-ratings of IC show a much more stable and consistent etiology and developmental trajectory than do lab-based assessments. Researchers have also found genetic and environmental covariance between IC and externalizing behavior problems in toddlerhood (Gagne, Saudino \& Asherson, 2011) and school age (Lemery-Chalfant, Doelger, Goldsmith, 2008). We examined the development and etiology of IC and ADHD from a multi-method perspective longitudinally from early childhood to adolescence. Participants included 101-245 MZ and 150-424 DZ twin pairs from the Wisconsin Twin Project. Mothers rated IC in todderhood (TBAQ) and first grade (CBQ), and mother ratings of DISC-ADHD symptoms were collected in first grade and again in early adolescence. Phenotypic correlations between IC and ADHD ranged from -.20 to -.68 . MZ correlations exceeded DZ correlations, indicating genetic influences. Cross-twin, cross-trait correlations for $\mathrm{MZ}$ twins exceeded those for $\mathrm{DZ}$ twins suggesting genetic covariance between IC and ADHD. Initial bivariate Cholesky decomposition models of toddler IC and first grade ADHD, and first grade IC and early adolescent ADHD yielded genetic and nonshared environmental variances and covariances (genetic correlations between IC and ADHD ranged from -.19- -.24). Results show that toddler IC is phenotypically and etiologically associated with ADHD in first grade, as is first grade IC and early adolescent ADHD. Based on these findings, early IC can be considered a genetic risk factor for later ADHD symptoms. Future analyses will include laboratory-based behavioral assessments of IC in first grade.

\section{Genetic and neurobiological influences on internalizing characteristics in binge drinkers}

Megan Cooke, Virginia Commonwealth University, United States; James Bjork, Virginia Commonwealth University, United States; Kenneth Kendler, Virginia Commonwealth University, United States; Danielle Dick, Virginia Commonwealth University, United States

Previous research has hypothesized a developmental pathway to alcohol use disorder characterized by internalizing symptoms (Hussong et al., 2011; Psychol Addict Behav. 25, 390-404). This pathway has been predominantly researched and characterized in terms of selfreported phenotypic characteristics. However, less work has been done to determine whether individuals in this pathway are distinct in their underlying biological processes. The majority of gene finding studies treat alcohol misusers as a homogeneous group ignoring the possibility of potential genetic subtypes that correlate with the phenotypic subtypes described in the literature. To help fill this gap, the 
current study uses 3,079 regular binge drinkers from an ethnically diverse university-wide longitudinal study (Dick et al., 2014; Front Gen. 5:47) to explicitly examine internalizing characteristics in the context of heavy drinking. Due to the ethnic diversity of the sample, analyses were conducted separately by ancestry, and then meta-analyzed (Webb et al., 2017; Front Genet. 8:30). Genome-wide association analyses showed five genome-wide significant markers in the RPH3AL gene $\left(\mathrm{p}<5.0 \times 10^{-8} \mathrm{q}=0.08\right)$. An additional seven markers in this gene were marginally significant $\left(p=6.61 \times 10^{-8}\right.$ $\left.2.38 \times 10^{-7}, \mathrm{q}=0.08-0.14\right)$. These markers were neither genome-wide significant nor marginally significant in genome-wide association analyses of internalizing characteristics in the full sample which includes individuals with lighter drinking patterns. This suggests that this gene is uniquely associated with the combination of internalizing characteristics and heavy drinking reiterating the importance of incorporating phenotypic heterogeneity in genetic analyses. A portion of this sample (current $\mathrm{N}=36$ ) is undergoing a neuroimaging protocol. Forthcoming analyses will examine associations between RPH3AL and relevant neuroimaging outcomes as well as the potential mediating role of brain activation in the association between RPH3AL and internalizing binge drinking.

\section{Maternal antidepressant use during the first trimester of pregnancy and offspring neurodevelopmental problems}

Ayesha Sujan, Indiana University, United States; Martin Rickert, Indiana University, United States; A. Sara Oberg, Harvard T.H. Chan School of Public Health, United States; Patrick Quinn, Psychological \& Brain Sciences, Indiana University, United States; Sonia Hernández-Díaz, Indiana University, United States; Catarina Almqvist, Karolinska Institutet, Sweden; Paul Lichtenstein, Karolinska Institutet, Sweden; Henrik Larsson, Karolinska Institutet, Sweden; Brian D’Onofrio, Indiana University, United States

Previous studies have found associations between maternal antidepressant use during pregnancy and offspring neurodevelopmental problems, including autism spectrum disorder (ASD) and attentiondeficit/hyperactivity disorder (ADHD). However, these studies may not have adequately accounted for confounding factors, such as maternal depression. The present study of 1,580,629 Swedish offspring used two sources of information on maternal antidepressant use (self-reports and dispensation records) and four designs to account for confounding factors to assess associations between maternal firsttrimester antidepressant use and offspring ASD and ADHD. The designs were (1) statistical controls to adjust for measured pregnancy, maternal, and paternal characteristics; (2) sibling comparisons to account for unmeasured genetic and environmental factors that make siblings similar; (3) timing-of-exposure comparisons to account for factors related to maternal antidepressant treatment around the time of pregnancy; and (4) paternal comparisons as another approach to account for familial confounding. In the population, first-trimester antidepressant exposure based on maternal report was associated with ASD $(\mathrm{HR}=2.0,95 \% \mathrm{CI},[1.8-2.3])$ and $\mathrm{ADHD}(\mathrm{HR}=2.2,95 \% \mathrm{CI}$, [2.0-2.4]). However, in models that compared siblings while adjusting for pregnancy, maternal, and paternal traits, first-trimester exposure was not associated with ASD $(\mathrm{HR}=0.8,95 \%$ CI [0.6-1.1]) or ADHD (HR $=1.0,95 \%$ CI [0.8-1.3]). For both ASD and ADHD, the associations with maternal antidepressant dispensations before but not during or after pregnancy did not statistically differ significantly from the associations with first-trimester maternal dispensations. Paternal first-trimester dispensations were associated with ASD $(\mathrm{HR}=$ 1.3, 95\% CI [1.1-1.6]) and ADHD (HR=1.7, 95\% CI [1.4-2.2]). Results from the present study suggest that maternal first-trimester antidepressant use does not increase the risk of offspring ASD and ADHD. These findings can help pregnant women and their doctors more accurately weigh the risks and benefits of treating maternal depression early in pregnancy with antidepressants.

\section{The genetic and environmental associations between cannabis use and annual income}

Nathan Gillespie, Virginia Commonwealth University, United States; Steven Aggen, Virginia Commonwealth University, United States; Michael Neale, Virginia Commonwealth University, United States; GunPeggy Knudsen, Norwegian Institute of Public Health, Norway; Nikolai Czajkowski, University of Oslo, Norway; Ragnar Nesvag, Norwegian Institute of Public Health, Norway; Eivind Ystrom, Norwegian Institute of Public Health, Norway; Kenneth Kendler, Virginia Commonwealth University United States; Ted ReichbornKjennerud, Norwegian Institute of Public Health, Norway

Background: Cannabis use is linked to a number of maladaptive psychiatric and psychosocial outcomes including reduced income. Although familial aggregation in cannabis use and income can be explained by a combination of genetic and shared environmental factors, the biometrical genetic association between cannabis and income have not been explored.

Aim: What are genetic and environmental associations between cannabis use and annual income?

Methods: Subjects were 1,419 twins ( $\mu$ age $=28.2$ years) from the Norwegian Institute of Public Health Twin Panel with cannabis and income data. Measures of lifetime cannabis use were obtained in a face-to-face interview in 1999-2004 as part of a population-based study of mental health. This included assessments of lifetime cannabis use and cannabis use disorder based on DSM-IV abuse and dependence criteria, including withdrawal and craving. Annual income from 1993 to 2014 was obtained from The Income Registry through Statistics Norway. Cannabis and income data were merged based on unique national identification numbers granted to all Norwegians at birth.

Results: Multivariate analyses revealed significant genetic and environmental associations between cannabis use and annual income. Discussion: Results and implications to be discussed.

\section{The genetic and environmental influences on parenting across childhood and adolescence}

\section{Elizabeth Shewark, United States}

This symposium uses complementary methods across three papers to examine genetic and environmental influences on parenting. The first paper examines how genetic influences on children's negative emotionality may evoke warm and/or hostile parenting responses from mothers and fathers using a parent-offspring adoption design. The findings suggest that children play an important evocative role in parent-child interactions, and provide evidence that a portion of this association is likely heritable. The second paper examines the relatively understudied role of positive emotions in the parent-child dyad using a sample of toddler-aged twins. Results suggest an association between child positive emotion and maternal warmth and affection that is best explained by shared environmental influences. The third paper explores the relation between parent internalizing symptoms and negative parenting of their adolescent child using a sample of twin parents of an adolescent. Results indicate that the correlation between internalizing symptoms and parental negativity was 
explained by genetic and nonshared environmental influences, suggesting that, similar genetic influences contribute to both internalizing symptoms and negative parenting. The discussant, an expert in genetic influences on family processes, will discuss the importance of examining emotions and individual characteristics within the parentchild dyad, as well as the advantages of research that considers family relationships as interrelated subsystems that are influenced by genetic factors.

\section{An epigenomewide association study (EWAS) on alcohol consumption: a monozygotic twin study}

Yun Zhu, University of Florida, United States; Eric Strachan, University of Washington, United States; Emily Fowler, University of Washington, United States; Tamara Bacus, University of Washington, United States; Peter Roy-Byrne, University of Washington, United States; Jinying Zhao, University of Florida, United States

Alcohol consumption has been associated with many adverse health outcomes, but the mechanisms through which alcohol exerts its effect are not fully understood. Epigenetic mechanisms such as DNA methylation and resulting gene expression may underlie this linkage. Here we conducted genomewide DNA methylation (using Illumina HumanMethylation450 BeadChip) and gene expression (by Illumina HumanHT-12 v4 Expression BeadChip) analysis using peripheral blood monocytes isolated from 47 monozygotic (MZ) twin pairs. A total of $392 \mathrm{CpG}$ probes, clustered into 13 differentially methylated regions (DMRs), were found to be significantly associated with alcohol consumption. These regions included genes known to be related to alcohol drinking, e.g., GABBR1, GABARAP, GBX2, TDRD1, UNK, POLR3G and ZNF195. In addition, we identified 173 cis-and 288 trans-regulating genes associated with alcohol consumption. The identified alcohol-related DMR genes were enriched in GO terms such as alcohol oxidase activity, fatty alcohol metabolic process, and one-carbon metabolic process. Differential connectivity patterns in the co-methylation networks were also observed for genes involved in these three GO terms. Our results demonstrated that alcohol consumption alters DNA methylation and gene expression, independent of genetic and other potential confounding factors. These findings provide novel insight into biological mechanism involved in alcohol use, and may also unravel novel epigenetic pathways through which alcohol drinking contributes to alcohol-related diseases.

\section{Teaching motivation and altruism: a Japanse twin study}

Juko Ando, Keio University, Japan; Kai Hiraishi, Keio University, Japan

Teaching behavior is a kind of altruistic behavior to share knowledge with community members. Although genetic and environmental effects of altruism have been investigated (e.g. Rushton et al., 1986; Gregory et al., 2009), motivation to teach and its relation to altruism are not examined from a behavioral genetic perspective. In the current study, genetic and environmental structure of altruism and teaching motivation were investigated with 623 pairs of Japanese adult twins (132 male MZ, 327 female MZ, 31 male DZ,93 female DZ, 40 opposite sex DZ), using the Self-Report Altruism Scale Distinguished by the Recipient (SRAS-DR) (Oda et al., 2013) which distinguishes three aspects of altruism according to recipients (family members, friends or acquaintances, and strangers, corresponding to kinship, indirect reciprocity, and direct reciprocity respectively). Three aspects of altruism were moderately genetic with no shared environment. Teaching motivation consisted of two factors; supportive motivation and enlightenment motivation. Although enlightenment motivation showed genetic effect, supportive motivation showed substantial shared environmental effects. The results also indicate altruism and teaching motivation were mediated by genetic factors.

\section{Examining the shared genetic architecture of risk tolerance and related behaviors}

Edward Kong, Harvard University, United States; Adam Auton, 23andMe, United States; Pierre Fontanillas, 23andMe, United States; Sonia Jain, University of California, San Diego, United States; Chiayen Chen, Massachussetts General Hospital, United States; Murray Stein, University of California, San Diego, United States; Lars Bertram, University of Lübeck, Germany; Peter Eibich, University of Oxford, United Kingdom; Christina Lill, University of Lübeck, Germany; Gert Wagner, Max Planck Institute for Human Development, Germany; Anke Hammerschlaag, Vrije Universiteit Amsterdam, Netherlands; Danielle Posthuma, Vrije Universiteit Amsterdam, Netherlands; Erdogan Taskesen, Vrije Universiteit Amsterdam, Netherlands; Andrew Conlin, University of Oulu, Finland; Ville Karhunen, University of Oulu, Finland; Minna Männikkö, University of Oulu, Finland; Rauli Svento, University of Oulu, Finland; Michel Nivard, Vrije Universiteit Amsterdam, Netherlands; Dorret Boomsma, Vrije Universiteit Amsterdam, Netherlands; Abdel Abdellaoui, Vrije Universiteit Amsterdam, Netherlands; Conor Dolan, Vrije Universiteit Amsterdam, Netherlands; Harriet de Wit, University of Chicago, United States; Abraham Palmer, University of California, San Diego, United States; James Mackillop, McMaster University, Canada; Sandra SanchezRoige, University of California, San Diego, United States; Albert Hofman, Erasmus University, Rotterdam, Netherlands; M. Arfan Ikram, Erasmus Medical Center, Netherlands; Henning Tiemeier, Erasmus Medical Center, Netherlands; André Uitterlinden, Erasmus Medical Center, Netherlands; Frank Van Rooij, Erasmus University, Rotterdam, Netherlands; Patrick Groenen, Erasmus University, Rotterdam, Netherlands; Roy Thurik, Erasmus University, Rotterdam, Netherlands; Jonathan Beauchamp, University of Toronto, Canada; Daniel Benjamin, University of Southern California, United States; Pietro Biroli, University of Zurich, Switzerland; David Cesarini, New York University, United States; Ronald De Vlaming, Vrije Universiteit Amsterdam, Netherlands; Mark Fontana, University of Southern California, United States; Richard Karlsson, Linnér Vrije Universiteit Amsterdam, Netherlands; Philipp Koellinger, Vrije University, Netherlands; David Laibson, Harvard University, United States; Maël Lebreton, Universiteit van Amsterdam, Netherlands; James Lee, University of Minnesota, Twin Cities, United States; Fleur Meddens, Vrije Universiteit Amsterdam, Netherlands; Gerardus Meddens, Team Loyalty BV, Netherlands; Aysu Okbay, Vrije Universiteit Amsterdam, Netherlands; Niels Rietveld, Erasmus University, Rotterdam, Netherlands; Patrick Turley, Broad Institute of MIT and Harvard, United States; Robbee Wedow, University of Colorado Boulder, United States; Jake Gratten, The University of Queensland, Australia; Magnus Johannesson, Stockholm School of Economics, Sweden; Robert Karlsson, Karolinska Institutet, Sweden; Patrik K. E. Magnusson, Karolinska Institutet, Sweden; Yanchun Bao, University of Essex, United Kingdom; Meena Kumari, University of Essex, United Kingdom; Melissa Smart, University of Essex, United Kingdom; Jon White, University College London, United Kingdom; Peter Joshi, University of Edinburgh, United Kingdom; James Wilson, University of Edinburgh, United Kingdom; 
David Clark, University of Edinburgh, United Kingdom; Laura Buzdugan, University of Zurich, Switzerland; Ernst Fehr, University of Zurich, Switzerland; Urs Fischbacher, University of Konstanz, Germany; Gregor Hasler, University of Bern, Switzerland; Carlos Morcillo-Suarez, Universitat Pompeu Fabra, Spain; Gerard Muntané, Universitat Pompeu Fabra, Spain; Arcadi Navarro, Universitat Pompeu Fabra, Spain; Klaus Schmidt, University of Munich, Germany; Daniel Schunk, Johannes Gutenberg University, Germany; Matthias Sutter, University of Cologne, Germany

Risk tolerance is an important behavioral parameter in the social sciences. It underpins models of individual choice in many domains, from the propensity to engage in risky behaviors to labor supply and insurance decisions. To examine the shared genetic etiology of general and domain-specific risk phenotypes, we leverage results from several large GWAS of risk tolerance and risky behaviors. First, we measure the SNP heritability of these traits. Next, we examine the genetic correlation between these risk phenotypes and various other traits. We find significant genetic correlations with self-employment and several neuropsychiatric conditions. For traits that share a significant genetic background with risk tolerance, we conduct proxyphenotype lookup analyses and identify additional association loci. Lastly, we construct a polygenic score for general risk tolerance and demonstrate that it is predictive of personality traits and real world behaviors, such as openness and entrepreneurship.

\section{Co-twin control models: assessing bias from measured and unmeasured confounders}

Gretchen Saunders, University of Minnesota - Twin Cities, United States; Matt McGue, University of Minnesota, United States

Between-within models are increasingly used to add causal evidence to observational data. In particular, co-twin control models, a subset of between-within models, make use of discordant twin pairs to evaluate whether the relationship between an exposure and outcome is consistent with a causal interpretation. While the popularity of these models is increasing, they have not been fully explored methodologically. Prior research has shown that bias can be introduced into model estimates through unmeasured confounders that are not shared within a twin pairsup1/sup. The amount of bias is a function of the correlation in exposure and the correlation in the unmeasured confounder within twin pairs. The current study makes use of simulation and analytic proofs to explore whether this bias can be reduced by the inclusion of a measured confounder. It can be shown that both the crude and within-pair estimates in a model including a measured confounder do indeed reduce the bias introduced by a non-shared confounder within twin pairs. The reduction in bias is a function of the individual-level correlation between the unmeasured and measured confounder. Interpretation of the withinpair effect, as an estimate of the causal effect of exposure on outcome, in co-twin control models is discussed.

\section{References}

Frisell T, Öberg S, Kuja-Halkola R, \&Sjölander A. (2012). Sibling comparison designs: Bias from non-shared confounders and measurement error. Epidemiology, 23, 713-720.

\section{Initial phase of the GSCAN GWAS project}

Mengzhen Liu, University of Colorado - Boulder, United States; GSCAN, United States
Alcohol and tobacco use are complex behavioral phenotypes that show highly polygenic inheritance. Although genome-wide analyses have found a small number of common variants associated with these phenotypes, there likely remain many additional associated loci to be found, which will provide clues to biological mechanisms involved in addiction. GWAS \& Sequencing Consortium of Alcohol and Nicotine use (GSCAN) is an international consortium to conduct genetic association analyses across many studies and hundreds of thousands of individuals. Our current freeze of 29 studies have provided summary statistics from association studies with six smoking/alcohol related phenotypes: cigarettes per day $(\mathrm{N}=256,658)$, regular versus never smoker $(\mathrm{N}=991,257)$, smoking cessation $(\mathrm{N}=420,466)$, age of initiation of regular smoking $(\mathrm{N}=260,513)$, drinks per week $(\mathrm{N}=$ $632,043)$ and drinker versus non-drinker $(\mathrm{N}=688,077)$. Preliminary results indicate discovery of dozens of novel independent loci across the six traits, as would be expected for samples of this size. More refined and finalized results will be presented. We will also present secondary analyses using these results to generate polygenic risk scores and genetic correlations between the six addiction phenotypes.

\section{Social class and healthy aging: what we can learn from IGEMS}

Matt McGue, University of Minnesota, United States; Inge Petersen, University of Southern, Denmark, Denmark; Shandell Pahlen, University of California, Riverside, United States; IGEMS Consortium, University of Southern California, United States

Many important health outcomes are associated with social class. Yet how social class comes to be associated with health outcomes has been the subject of much debate and controversy within the field of social medicine. Social causation models account for the association by hypothesizing that social standing exerts a causal influence on health. Alternatively, social selection models posit that the association arises through reverse causation, that health influences social class achievement. Although twin and family studies cannot fully resolve social causation from social selection, these methods have been instrumental to testing some of the key tenets of the two models. Several features of IGEMS makes it very well suited to exploring mechanisms underlying the association of social class with late-life health. Specifically, IGEMS includes intergenerational social class data on large numbers of twins, which allows a differentiation between effects attributable to social class of origin from those attributable to achieved social class. Preliminary findings from IGEMS will be presented to illustrate the utility of behavioral genetic methods for understanding social class effects.

\section{Irving Isadore Gottesman: the Minnesota years}

\section{Matt McGue, University of Minnesota, United States}

You would not have known it in talking with him, especially if the conversation turned to sports team allegiances, but Irv Gottesman was not born in Minnesota. Yet when given the chance, Irv always chose to call Minnesota his home. And his life afforded three such opportunities to show his commitment to his adopted home: In 1956 when he chose a Ph.D. training program, in 1966 as a young Assistant Professor, and in 2001 when he formally 'retired' from academia. Over six decades, Irv had an extraordinary impact in shaping the Department of Psychology at the University of Minnesota. He started an interdisciplinary training program in behavioral genetics, fostered the genetics training and interests of a young Assistant Professor 
named Tom Bouchard, and mentored two generations of behavioral genetics pre-doctoral and post-doctoral researchers. But it would be misleading to characterize Irv's relationship with Minnesota as unidirectional. Minnesota had as much of an effect on Irv as he had on it. Minnesota helped Irv become the type of psychologist he was, a hardheaded empiricist who was deeply committed to investigating the origins of individual differences in behavior no matter where that inquiry would take him.

\section{A whole-brain atlas of genetic effects on psychiatric symptomology}

Alexander Hatoum, Institute for Behavioral Genetics, United States; Andrew Reineberg, University of Colorado Boulder, United States; Harry Smolker, University of Edinburgh, United States; John Hewitt, University of Colorado Boulder, United States; Naomi Friedman, University of Colorado Boulder, United States

Anatomical variation in the brain is a candidate endophenotype for psychiatric symptomology. Initial work has linked variation (e.g., cortical thickness, surface area) in anatomical regions of interest (ROIs), to most psychiatric syndromes, with Insula and dorsolateral prefrontal cortex often predictive of psychiatric outcomes. Although promising initial descriptions, this work does not take advantage of the high dimensionality of fMRI images, i.e. vertex-wise (smallest possible picture of brain area) estimates. Furthermore, genetic effects may vary within predefined ROIs, and relative differences in heritability between ROI's may be overestimated. In this study, we map the genetic effects on anatomical measures prediction of symptoms to create whole-brain atlases of genetic effects on psychiatric disorders. We use a subset of 270 twins from the Colorado Longitudinal Twin Study, with replication from a comparable twin sample from the Human Connectome Project. Initial results of genetic estimates across 148 ROI's show left hemisphere structures tend to be under larger genetic influence than right structures. As such, bivariate brain-behavior biometrical models show genes influencing left structures are more predictive of psychiatric variation. Broadly, psychiatric variation was associated with genes influencing larger somatosensory regions and smaller insula regions. The genetic influences on brain regions that most highly predict one psychiatric disorder also predicted other psychiatric disorders, suggesting a possible underlying anatomical genotype for psychiatric behaviors. Prior to presentation, we will map these predictions for multiple psychiatric symptoms at the vertex-wise level. Our results suggest that anatomical measures are a key endophenotype for the association between genes and disorders.

\section{The impact of variation in twin relatedness on estimates of genetic and environmental influences}

Chang Liu, Pennsylvania State University, United States; Peter Molenaar, The Pennsylvania State University, United States; Jenae Neiderhiser, The Pennsylvania State University, United States

By taking advantage of the natural variation in genetic relatedness among identical (monozygotic: MZ) and fraternal (dizygotic: DZ) twins, twin studies are able to estimate genetic and environmental contributions to complex human behaviors. Recently concerns have been raised about the accuracy of twin studies in light of findings of genetic and epigenetic changes in twins. One of the concerns raised is that MZ twins are not $100 \%$ genetically (and epigenetically) similar because they show variations in their genomes and epigenomes leading to inaccurate estimates of heritability. The current study presents findings from a simulation study that examined the degree of bias in estimates of heritability and environmentality when the genetic similarity of MZ twins differs from 1.00 and when the genetic similarity of DZ twins differs from .50. The findings suggest that in the standard biometric model when $\mathrm{MZ}$ or DZ twin similarity differs from 1.0 to .50, respectively, the variance that should be attributed to genetic influences is instead attributed to nonshared environmental influences, thus deflating the estimates of genetic influences and inflating the estimates of nonshared environmental influences. Although estimates of genetic and nonshared environmental influences from the standard biometric model were found to deviate from "true" values, the bias was usually smaller than 10 percentage points indicating that the interpretations of findings from previous twin studies are mostly correct. In contrast to the fixed genetic factor correlations in the standard biometric model, the alternative model sets the genetic factor correlations of MZ and DZ twins to be free parameters that need to be estimated. The findings suggest that in the alternative model, all the estimates of MZ and DZ twins' genetic factor correlations and genetic, shared environmental and nonshared environmental influences are within $95 \%$ confidence intervals about the "true" parameter values used in generating the data.

\section{Examining the effects of genetic, prenatal illicit drug exposure, and postnatal rearing environment on child school age outcomes}

Chang Liu, Pennsylvania State University, United States; Leslie Leve, University of Oregon, United States; Daniel Shaw, University of Pittsburgh, United States; Jody Ganiban, The George Washington University, United States; David Reiss, Yale University, United States; Misaki Natsuaki, University of California, Riverside, United States; Jenae Neiderhiser, The Pennsylvania State University, United States

Although many studies have explored the impact of prenatal illicit drug exposure (PDE) on child outcomes, non-PDE risks such as genetic influences and rearing environment that are correlated with PDE make it difficult to distinguish the effects of PDE from other risks on child outcomes. Identifying the longitudinal effects of PDE on child outcomes, while controlling for other risks, helps to clarify the mechanisms underlying PDE and child development. Using data from the Early Growth and Development Study (N=561) we examined effects of PDE on child negative reactivity at 18 months and later problem behaviors at 7 years after accounting for child sex, heritable risks, and negative parenting. Heritable risks for externalizing and internalizing problems were created using birth mothers' (BM) lifetime psychiatric diagnoses, current symptoms, age of onset of substance use initiation and first-degree parents' lifetime psychiatric diagnoses. BM reported on their own illicit drug use during pregnancy at 5 months postpartum using a life-history calendar method. A composite of child negative reactivity was created using adoptive parents' reports of child emotional reactivity (CBCL), anger, fear (TBAQ) and fussy/difficult temperament (ICQ) at 18 months. Child problem behaviors were assessed with adoptive parents' (AP) reports on the CBCL externalizing and internalizing problems at age 7. A composite of negative parenting was created using AP reports of inconsistent discipline, corporal punishment, and overall hostility at child age 4.5 years. Results indicated that PDE was negatively associated with child negative reactivity at 18 months $(B=-.50, p$ $=.05$ ). Furthermore, heritable risk for internalizing problems, PDE, and child negative reactivity were positively associated with later internalizing and externalizing problems, but only for boys $(B=-4.05$, $\mathrm{p}<.01$, and $B=-3.71, \mathrm{p}=.06$ ). For children with PDE, higher levels of negative parenting was associated with increases in children's externalizing problems at age $7(\beta=-49.86, p=.04)$. 
Reproductive success versus fitness: testing the genetic correlation between number of children and grandchildren in a range of preindustrial societies

Brendan Zietsch, University of Queensland, Australia; Abdel Abdellaoui, Vrije Universiteit Amsterdam, Netherlands; Drew Bailey, University of California, Irvine, United States; Robert Walker, University of Missouri, United States; Karin Verweij, Radboud University, Netherlands

Evolutionary fitness is usually measured by number of offspring, but this measure may not reflect the genetic contribution to subsequent generations (i.e. true fitness) if offspring vary in their own ability to survive and reproduce. Trade-offs between offspring quantity and quality are especially plausible in humans, given the intense and extended care children require to survive to reproductive age. Using number of children as a proxy for fitness may therefore yield incorrect inferences about selection pressures and projections of evolutionary change. A previous paper (Zietsch et al., 2014, PNAS) showed that the same genes influence both number of children and grandchildren in industrialised Sweden, indicating that number of children does adequately measure fitness in this sample. However, the conclusions from this study may not apply to traditional, natural-fertility societies with higher birth and mortality rates and often scarce or unreliable resources. To test this, we investigated the genetic variance of and covariance between the number of offspring and grandoffspring of individuals from 46 small-scale traditional societies with a total $\mathrm{N}>$ 45,000 . The findings, to be discussed, have important implications regarding our understanding of the process of evolution in humans.

\section{Can we validate twin studies? A census-based heritability estimate of educational achievement}

Stéphanie van den Berg, University of Twente, Netherlands; Inga Schwabe, Tilburg University, Netherlands; Luc Janss, Aarhus University, Denmark

For most phenotypes, the amount of variance explained by SNPs is generally lower than the amount of additive genetic variance estimated in twin studies. This is also true for educational achievement. Twin-based estimates may be biased because of self-selection and differences in cognitive ability between twins and the rest of the population. Here we compare twin registry based estimates with a census-based heritability estimate, sampling from the same birth cohort population and the same standardized measure for educational achievement. We analyzed 893,127 scores on a Dutch test administered at the end of primary school, from the years 2008-2014. For genetic inference, we used pedigree information to construct an additive genetic relationship matrix. We analyzed the data using a Bayesian linear mixed model, including important covariates. Corrected for these covariates, this resulted in an estimate of $83 \%$, which is even higher than based on twin studies using the same cohort and measure. We therefore conclude that the genetic variance not tagged by SNPs is not an artefact of the twin method itself.

\section{Investigating intergenerational associations: revisiting the children-of-twins design II}

Fruhling Rijsdijk, King's College London, MRC Social, Genetic and Developmental Psychiatry Centre, Institute of Psychiatry, Psychology and Neuroscience, London, UK, United Kingdom; Eivind
Ystrom, Norwegian Institute of Public Health, Norway; Line C. Gjerde, Norwegian Institute of Public Health, Norway; Espen Eilertsen, Norwegian Institute of Public Health, Norway; Tom McAdams, King's College London, United Kingdom

Datasets comprising twins and their children can be a useful tool for those interested in understanding the nature of intergenerational associations between parent and offspring phenotypes. For example, by using such data it is possible to ask whether parent-child associations can be explained via genetic transmission, and/or whether associations remain significant after accounting for genetic relatedness between parent and child. By applying structural equation models (SEMs) to children-of-twins (CoT) data, it is possible to quantify the relative importance of genetic versus non-genetic (social) pathways. In the past, extensions of this model were presented which include bidirectional effects between parent and child phenotypes (i.e. parent-to-child versus child-to-parent effects). This was accomplished by simultaneously modelling CoT data with children-as-twins-with parent data on the same phenotypes. This extended two-sample model (ECoT, Narusyte et al. 2008) is an adaptation of the bidirectional models first suggested by Heath et al. (1993), and explored more recently by Olivares et al. (2016). The purpose is to use cross-sectional twin/family data to ask questions about the direction of causation between two phenotypes. The ECoT model proved to have some shortcomings. In this talk I will explore the SEMs that have been used to analyse ECoT data, highlighting some of the limitations that these models have, and how they can, to a certain extent, be solved by using an extension including multiple offspring per parent in the CoT design. I will demonstrate the power and utility of these models using simulated data and real data from the Intergenerational Transmission of Risk Project (a subsample of the Norwegian Mother and Child Cohort Study).

\section{References}

Narusyte J., Neiderhiser J.M., D’Onofrio B.M., Reiss D., Spotts E. L., Ganiban J., \& Lichtenstein P. (2008). Testing different types of genotype-environment correlation. Developmental Psychology, 44, 1591-1603.

Heath A.C., Kessler R. C., Neale M. C., Hewitt J. K., Eaves L. J. \& Kendler K. S. (1993). Testing hypotheses about Direction of Causation. Behavior Genetics, 23(1), 29-50.

Olivares E.L., Kendler K.S., Neale M.C., Gillespie N.A. (2016). The genetic and environmental association between parental monitoring and risk of cannabis.. Twin Research and Human Genetics, 19 (4), 297-305.

Fatigue symptoms in relation to neuroticism, anxietydepression, and musculoskeletal pain. A longitudinal twin study

Olav Vassend, University of Oslo, Norway; Christopher Nielsen, Norwegian Institute of Public Health, Oslo; Department of Pain Management and Research, Oslo University Hospital, Norway; Espen Røysamb, University of Oslo Norway; Nikolai Czajkowski, University of Oslo, Norway

Background: Fatigue symptoms are associated with a variety of syndromes and diseases, but in many cases no specific underlying disease activity or psychiatric condition can be found. Both common fatigue conditions and the more serious chronic fatigue syndrome (CFS) have been shown to be linked to pain symptoms, particularly musculoskeletal (MS) pain, and to negative affect symptoms such as anxiety and depression. However, the nature of this relationship is 
poorly understood. In the present study the genetic and environmental association between anxiety-depression, MS pain and fatigue was examined, and the role of neuroticism as a shared risk factor that may possibly explain the co-occurrence between these phenotypes was investigated in a combined cross-sectional and longitudinal twin design.

Methods: The sample consisted of 746 monozygotic (MZ) and 770 dizygotic (DZ) twins in the age group of 50-65 (mean $=57.11$ years, $\mathrm{SD}=4.5$ ). Using Cholesky modeling, genetic and environmental influences on neuroticism, anxiety-depression, MS and fatigue symptoms, and the associations among these phenotypes, were determined.

Results: The best-fitting biometric models included additive genetic and individual-specific environmental effects. All four phenotypes were moderately to highly influenced by genetic factors [heritability $\left.\left(\mathrm{h}^{2}\right)=0.40-0.53\right]$. Furthermore, while there was a considerable overlap in genetic risk factors between the four phenotypes, a substantial proportion of the genetic risk shared between anxiety-depression and fatigue, and between MS pain and fatigue, was independent of neuroticism. The independent genetic influence of MS pain on fatigue was substantial, and the genetic correlation between the two phenotypes was particularly (i.e., 0.88).

Conclusion: Evidence for a common underlying susceptibility to report fatigue symptoms, genetically linked to neuroticism, anxietydepression, and MS pain, was found. Both unique and pleiotropic effects appear to be involved in the genetic architecture of the phenotypes.

\section{The etiological link between clinical psychiatric diagnoses and continuous variation in related traits: evidence from a twin study}

Mark Taylor, Karolinska Institutet, Sweden; Joanna Martin, Karolinska Institutet, Sweden; Sebastian Lundström, University of Gothenburg, Sweden; Paul Lichtenstein, Karolinska Institutet, Sweden

Psychiatric disorders are often argued to represent the extreme manifestation of continuous traits, which present to varying degrees of severity throughout the general population. As such, one hypothesis is that genetic risk factors for such disorders are also associated with traits characteristic of these disorders. In this study, we utilized a large twin study in which participants have been comprehensively screened for psychiatric disorders using continuous trait measures, and which has been linked with Swedish patient registries. Parents of approximately 14,000 twin pairs taking part in the Child and Adolescent Twin Study in Sweden (CATSS) completed scales assessing traits characteristic of autism spectrum disorders (ASD), attentiondeficit/hyperactivity disorder (ADHD), intellectual disability, anxiety disorders, depression, obsessive-compulsive disorder (OCD), and tic disorder when twins were aged 9 or 12. Information on clinical diagnoses of these seven conditions was extracted from the Swedish National Patient Register. We then applied an approach to twin analysis that involved jointly modeling liability to each dichotomous diagnosis and continuous variation in each trait, which estimated the genetic correlation between clinical diagnoses and continuous traits. Each dichotomous disorder and continuous scale was under significant genetic influence (disorder liability heritability $=36-94 \%$; trait heritability $=23-69 \%$ ). For six phenotypes, there was a significant genetic correlation between the dichotomous diagnosis and traits characteristic of the same disorder: genetic correlations $=.55$ (ADHD), .50 (intellectual disability), .40 (tic disorder), .38 (ASD), and .24 (anxiety disorders and depression). The genetic correlation between OCD and characteristic traits was weak and non-significant (.13).
Thus, our findings support the hypothesis that psychiatric disorders represent the extreme end of a continuous distribution of population traits.

\section{Genetic and environmental influences on workforce absence due to ill health: a life span study}

Karoline Seglem, Norwegian Institute of Public Health, Norway; Fartein Torvik, Norwegian Institute of Public Health, Norway; Line C. Gjerde, Norwegian Institute of Public Health, Norway; Espen Røysamb, University of Oslo, Norway; Kristian Tambs, Norwegian Institute of Public Health, Norway; Ragnhild Ørstavik, Norwegian Institute of Public Health, Norway

Objectives: It is a high political priority to maximize workforce participation in the working age population. Absence from the workforce due to physical and psychological ill health has huge societal and individual costs, economically and socially. The present study aims to gain more knowledge about the causes of individual variation, namely genetic and environmental influences, underlying medically certified workforce absence across the life span and whether there are differences between males and females in the type, magnitude and stability of these influences.

Methods: A population-based sample of 29257 twins born between 1926 and 1991 participating in the Norwegian Institute of Public Health Twin Panel was linked to registry data on medically certified workforce absence, including sickness absence, rehabilitation and disability, during the years 1992 to 2016 . Multivariate genetic modelling was performed on the number of absence days per life decade from participants' age 18 until 66.

Results: Preliminary findings indicate genetic stability and a tendency of increasing stability with age. Genetic stability between young adulthood (age 18-29) and old adulthood (age 60-66) was moderate. Heritability was moderate at all ages, but showed a decreasing tendency in males, whilst fluctuating in females. Environmental factors not shared with family members was the most influential factor across life and became increasingly stable with age, whereas shared environmental factors had none or negligible effects. Detailed analyses will be presented at the conference.

\section{Adverse family life events during pregnancy and ADHD symptoms in five year old offspring}

Mina Rydell, Karolinska Institutet, Sweden; Arvid Sjölander, Karolinska Institutet, Sweden; Eivind Ystrom, Norwegian Institute of Public Health, Norway; Henrik Larsson, Karolinska Institutet, Sweden

Adverse life events during pregnancy have been suggested to increase the risk of ADHD in the offspring, but the role of familiar confounding is unclear. The aim of this study was to clarify if prenatal exposure to adverse life events in the family is associated with ADHD symptoms in 5 year old children, and to assess if such an association remains after controlling for shared familial factors. The study is based on 34,751 children (including 6,427 siblings) whose mothers participated in the population-based Norwegian Mother and Child Cohort Study during pregnancy and at the child's age of five. During pregnancy, mothers reported whether they had experienced eight specific life events. ADHD symptoms were reported by parents at the child's age of five using the Conner's Parent Rating Scale-Revised: short form. Linear regression models were used to assess mean ADHD scores (range: 0-3) in the full cohort. To adjust for 
unmeasured familial confounding, fixed-effect models were used to compare mean ADHD scores among exposure-discordant siblings. Results showed that children exposed to adverse life events had higher ADHD scores at age five. The largest effect was observed for financial problems, and the weakest for having lost someone close (mean differences 0.10 [95\% CI 0.09-0.11] and 0.02 [95\% CI 0.010.04] respectively in adjusted models). Comparing exposure-discordant siblings resulted in attenuated estimates that were no longer statistically significant (e.g. mean difference for financial problems 0.03 [ $95 \% \mathrm{CI}-0.07$ to 0.02$]$ ). However, wide confidence intervals for specific life events hamper full interpretation of these results. These findings show that prenatal exposure to adverse life events in the family is associated with ADHD symptoms in 5 year old children, but most associations seem to be explained by familial confounding.

\section{Multivariate, multi-rater and multi-age GWAS of aggression and attention problems}

Michel Nivard, Vrije Universiteit Amsterdam, Netherlands; Hill Fung Ip, VU, Netherlands; EAGLE (Early Genetics And Lifecourse Epidemiology) Consortium, United Kingdom; ACTION, Aggression in Children: Unraveling gene-environment interplay to inform Treatment and InterventiON, Netherlands

Genetic effects contribute to variation in childhood aggression (AGG) and attention (ATT) problems. Analyses of repeated observations of AGG and ATT in childhood reveal substantial rank order stability (.41-.78), with a significant portion of this stability attributable to genetic factors (65\%) (Kan et al. JAACAP, 2013). Twin studies have shown that teacher self and parental ratings of AGG and ATT are genetically correlated, yet the rater specific variance also is heritable (Arsenault et al. J Child Psychol Psychiatry, 2003; Bartels et al. Behav Genet, 2003). GWA studies of AGG in children (Pappa et al. Neuropsychiatric Genetics, 2015; Middeldorp et al. JAACAP, 2016) have considered measures of AGG and ATT in univariate analyses, as have most other GWA meta-analyses projects (either in children or in adults). Twin studies have been at forefront of analyzing multivariate and longitudinal data, but GWA studies have only made use of these approaches in a limited way. The utilization of multiple measures on the same subject in GWA studies is non-trivial as it inflates type-1 error. To enable the multivariate genome wide study of the (developmental) genetic etiology of AGG and ATT, a multivariate metaregression model is presented. Our approach relies on univariate GWA of a longitudinal phenotype within a cohort, possibly assessed by multiple raters and the phenotypic correlations between traits within cohort (Nivard et al. Schizophr Bull, 2017). The multivariate aspect of the analysis is performed at the meta-analysis stage, omitting the need for multivariate modeling in individual cohorts. Our method enables the inclusion of dependent measures into a single analysis without inflating type-1 error. Our method further allows for estimation of age, rater or cohort specific effects for SNPs. We present a GWAS of AGG and ATT in repeatedly measured children at different ages (range 3-18) and by multiple raters (mother, father, teacher, self).

\section{Disentangling the effects of maternal depressive symptoms to child internalizing and externalizing problems: an extended children of twins study}

Line C. Gjerde, Norwegian Institute of Public Health, Norway; Espen Eilertsen, Norwegian Institute of Public Health, Norway; Tom
McAdams, King's College London, United Kingdom; Fruhling Rijsdijk, King's College London, MRC Social, Genetic and Developmental Psychiatry Centre, Institute of Psychiatry, Psychology and Neuroscience, London, UK, United Kingdom; Eivind Ystrom, Norwegian Institute of Public Health, Norway

Maternal depression has been found to be associated with internalizing and externalizing problems in preschool children. As both maternal depression and child internalizing and externalizing problems are influenced by genetic factors, it is likely that genes play a role in explaining intergenerational associations. Previous studies on parents and adolescent offspring typically find that genetic transmission is not important for explaining transmission of risk, indicating that mechanisms of causality may be operating. The extent to which associations between mothers and preschool children can be explained by genetic transmission has not yet been investigated in an extended children of twins study. The present study is based on data from a twin-family subsample of the Norwegian Mother and Child Cohort Study. Using structural equation models, we aim to investigate to what extent the effect of maternal depressive symptoms is transferred to the offspring through a genetic or direct environmental pathway. Maternal depressive symptoms and child internalizing and externalizing problems were measured when the children were 18, 36 and 60 months old. For optimal prevention of child mental health problems, it is important to understand whether the mechanisms of transmission are the same across time and for internalizing and externalizing problems. Preliminary results will be presented at the conference.

\section{Multi-rater and longitudinal GWAS of attention and aggression during development and the genetic correlations with adult psychiatric outcomes}

Hill Fung Ip, VU, Netherlands; Michel Nivard, Vrije Universiteit Amsterdam, Netherlands; ACTION Consortium Aggression in Children: Unraveling gene-environment interplay to inform Treatment and InterventiON, Netherlands; EAGLE Consortium, EArly Genetics and Lifecourse Epidemiology, Netherlands

We present the first results of a meta-analysis on childhood behavioral problems involving a collaboration between 17 international cohorts, form Europe (Finland, Germany, Netherlands, Spain, Sweden, UK), Australia, New-Zealand and the USA. The cohorts collaborate within the ACTION and EAGLE consortia, and are characterized by rich phenotyping in children, including repeated measures of aggressive behavior (AGG) and attention problems (ATT).

We analyzed longitudinal data of children and adolescents aged $3-$ 18 years, assessed by multiple raters (mother, father, teacher, and self) and multiple instruments. First, a series of univariate GWAS's was performed for every available combination of age and rater within each cohort, resulting in 3 to 36 analyses per cohort. Genotypes were imputed using the 1000 genomes Phase III reference set and mapped to the human reference genome GRCh37. Second, results were pooled into age by rater combinations (e.g. mother-rated aged 3-5, teacherrated $8-11$, etc.) that resulted in an excess of 10.000 observations and then meta-analyzed. We performed inverse variance weighted metaanalysis in $\mathrm{R}$ using custom code, correcting for the fact that repeatedly measured subjects were included in the analyses. We then used LD score regression to estimate the genetic correlation between the various rater by age combinations, across AGG and ATT. Furthermore, the genetic correlation between results of the meta-analyses and adult psychiatric outcome was estimated in order to determine the relative information found in ratings made by the various raters at different ages. Finally, we performed a meta-regression analysis 
across all GWAS's. In total, over 200 GWAS's were included in the meta-regression analysis, where the total number of observations equaled $\sim 526.000$ (the range of observations for individual age by rater by instrument GWAS was 309 to 10,812).

\section{Regulative, but not reactive, temperament traits and problem behavior are explained by the same genetic factors in Russian adolescents}

Georgy Vasin Psychological Institute of the Russian Academy of Education Russian Federation; Victoria Ismatullina Psychological Institute of the Russian Academy of Education Russian Federation; Marina Lobaskova Psychological Institute of the Russian Academy of Education Russian Federation; Sergey Malykh Psychological Institute of the Russian Academy of Education Russian Federation

Temperament traits influence how an individual reacts to everyday situations which over time forms their unique personality. The regulation of one's behavior is required to fit into societal norms and adapt to peers, which is especially important in adolescence. Contemporary research shows that environmental factors, such as harsh discipline and marital discord, can moderate the relationships between temperament traits and emotional and behavioral problems in children and adolescents (Richters, 2010, Sulik, 2013). It has also been shown that genetic factors have a moderate influence on both temperament (Malykh et al., 2005) and problem behavior (Klein R. \& Pine D., 2002). In our study we set out to disentangle the genetic and environmental factors that influence the relationships between temperament traits and emotional and behavioral problems. We based our study on the psychobiological approach to temperament developed by M. Rothbart is concerned with individual differences in both reactivity and self-regulation. Regulative processes are described through effortful control and its components: activation and inhibitory control. Reactive processes are broadly encompassed by negative emotionality and surgency. We used T. Achenbach's Youth Self Report (YSR) to assess emotional and behavioral problems and Rothbart's Adult Temperament Questionnaire (ATQ) to measure temperament traits. Our sample consisted of 372 twin pairs (164 MZ, 134 DZ, 74 opposite sex DZ). Participants were 14-19 years old, 54\% were female. Building on our previous study, we analyzed the relationships between all available ATQ and YSR scales in a standard bivariate twin study design. We found that the common variance between the effortful control scales and related problems (most notably delinquent and aggressive behavior as well as attention problems) was explained mostly by genetic factors. In contrast, the relationship between many reactive temperament traits and emotional and behavioral problems (for example, internalizing problems with shyness and fear) were explained mostly by shared environmental factors. These results indicate that even though both temperament traits and problem behavior can individually be explained by genetic factors, clearly those factors are only similar in a few cases. This study was supported by RFBR Grant No. 15-06-10724.

\section{Adolescent-limited cannabis use and developmental delay}

Maia Frieser, University of Colorado Boulder, United States; Robin Corley, University of Colorado Boulder, United States; John Hewitt, University of Colorado Boulder, United States; Christian Hopfer, University of Colorado Denver, United States; Tamara Wall, University of California, San Diego, United States; Chandra
Reynolds, University of California, Riverside, United States; Sally Wadsworth, University of Colorado Boulder, United States; Michael Stallings, University of Colorado Boulder, United States

Persistent heavy cannabis use in adolescence has been linked to a number of adverse outcomes, from deficits in neurocognitive functioning (Schweinsburg, Brown, \& Tapert, 2008) to poor educational performance (Lynskey \& Hall, 2000). These deficits have impacts that persist into later life, and have potentially serious negative consequences for adolescents who use cannabis regularly. These outcomes are also linked to delays in reaching adult developmental milestones (Brook, Zhang, Leukefeld, \& Brook, 2016). In the first stage of this study, we examined a group of 93 adolescent cannabis users who we will describe as "adolescent-limited". Adolescent-limited cannabis users are defined as heavy, daily cannabis users at maximum frequency of usage as teenagers, but by the age of 20 , their cannabis use has declined from daily use to sporadic use or total abstinence. We compare adolescent-limited cannabis users, persistent cannabis users (n over age $20=456$ ), and abstainers (n over age $20=$ 1,567). We examine potential delays in adult transitions, falling into primary domains of education, career, and family structure. The indicators of these domains include; highest level of education, number years of education, finishing high school, university or trade school, or post-undergraduate education, ever employed, job type, income, income source, fulltime employment, relationship status, relationship satisfaction, and number of children. These three groups of cannabis users will be compared to describe the potential consequences of using cannabis as an adolescent, even if usage ceases in adulthood. This sample is drawn from several longitudinal studies at the University of Colorado Boulder, through the Center for Antisocial Drug Dependence samples, and the Colorado Community and Longitudinal Twin Samples.

\section{References}

Brook, J. S., Zhang, C., Leukefeld, C. G., Brook, D. W. (2016). Marijuana Use from Adolescence to Adulthood: Developmental Trajectories and Their Outcomes. Social psychiatry and psychiatric epidemiology, 51(10), 1405-1415. doi:10.1007/s00127-016-1229-0.

Lynskey, M., \& Hall, W. (2000). The effects of adolescent cannabis use on educational attainment: a review. Addiction, 95(11), 1621-1630. doi:10.1046/j.1360-0443.2000.951116213.x.

Schweinsburg, A. D., Brown, S. A., Tapert, S. F. (2008). The Influence of Marijuana Use on Neurocognitive Functioning in Adolescents. Current drug abuse reviews, 1(1), 99-111. Retrieved from Ćhttp://www.ncbi.nlm.nih.gov.

\section{Early handwriting style has both genetic and environmental origins}

Zoe Reed, University of Bristol United Kingdom; Andrew Newell, University College London, United Kingdom; Bonamy Oliver, University of Sussex, United Kingdom; Lewis Griffin, University College London, United Kingdom; Oliver Davis, University of Bristol, United Kingdom

Handwriting is a ubiquitous physical trace of human behaviour. It is individual to a particular writer, even among twins (Srihari et al. 2008). We rely on the stability and distinctness of handwriting style to establish the validity of legal documents and to convict individuals of crimes. However, although we know much about the genetic and environmental origins of traits such as reading, language ability and spelling, we know little about the origins of writing, and nothing to date about the aetiology of handwriting style. Previous research has shown that teacher-rated writing ability has both genetic and 
environmental influences (Kovas et al., 2007), as do tests of writing ability such as creative sentence construction, writing fluency and handwriting speed (Olson et al., 2013). However, the complexity of handwriting style makes it difficult to study in a quantitative way. We used a novel machine learning approach (Newell \&amp; Griffin, 2014) to encode and compare handwriting samples from 4,349 sevenyear-old twins from the UK's Twins Early Development Study (TEDS). Structural equation modelling of the twin data showed statistically significant genetic $(8 \%)$, shared $(65 \%)$ and non-shared (26\%) environmental influence on handwriting style, with no apparent aetiological sex differences.

\section{References}

Srihari, S., Huang, C. \& Srinivasan, H., 2008. On the discriminability of the handwriting of twins. Journal of Forensic Sciences, 53 (2), pp. 430-446.

Kovas, Y., Haworth, C. M. A., Dale, P. S. \& Plomin, R., 2007. The genetic and environmental origins of learning abilities and disabilities in the early school years. Monogr Soc Res Child Dev 72(vii), pp. 1144.

Olson, R.K. et al., 2013. Genetic and environmental influences on writing and their relations to language and reading. Annals of dyslexia, 63(1), pp. 25-43.

Newell, A.J. \& Griffin, L.D., 2014. Writer identification using oriented Basic Image Features and the Delta encoding. Pattern Recognition, 47(6), pp. 2255-2265.

\section{Emotional overeating and depression in the Sri Lankan Twin Register}

Moritz Herle, University College London, United Kingdom; Carol Kan, King's College London, United Kingdom; Kaushalya Jayaweera, Institute for Research \& Development, Sri Lanka, Sri Lanka; Anushka Asikari, Institute for Research \& Development, Sri Lanka, Sri Lanka; Sisira Siribaddana, Rajarata University of Sri Lanka, Sri Lanka; Helena Zavos, King's College London, Institute of Psychiatry, Psychology \& Neuroscience, United Kingdom; Athula Sumathipala, Keele University, United Kingdom; Khalida Ismail, King's College London, United Kingdom; Matthew Hotopf, King's College London, United Kingdom; Janet Treasure, King's College London, United Kingdom; Fruhling Rijsdijk, King's College London, MRC Social, Genetic and Developmental Psychiatry Centre, Institute of Psychiatry, Psychology and Neuroscience, London, UK, United Kingdom

Associations between depression and obesity are often reported in epidemiological studies. Emotional overeating (EOE), the tendency to increase food consumption in response to sadness and stress, has been suggested as a link between depression and obesity. EOE therefore poses as a potential target for behavioural intervention in people with co-morbid depression and obesity. However, the aetiology of EOE is poorly understood, especially in the context of depression in populations with high levels of obesity. This study investigated the genetic and environmental contributions to depression and EOE in a SriLankan population, using genetic model-fitting analysis. 3561 twins and singletons in the Colombo Twin and Singleton Study-Phase 2 rated their EOE behaviour and depressive symptoms (Beck Depression Inventory). EOE and depression were significantly associated ( $\mathrm{r}$ $=.16$ Males, $\mathrm{r}=.17$ Females). A correlated factors twin model suggested distinct aetiology of EOE and depression between males and females. Heritability was higher for both EOE and depression in females (EOE h2 $=27 \%$, 95\% CI: $5-46 \%$; depression h2 $=26 \%, 95 \%$ CI: $2-48 \%$ ) than males (EOE h2 $=14 \%, 95 \%$ CI: $0-42 \%$; depression h2 $=8 \%, 95 \%$ CI: 2-8\%), while shared environmental factors were more important in males (EOE c $2=19 \%, 95 \%$ CI: $1-43 \%$; depression c2 $=20 \%, 95 \%$ CI: $4-36 \%$ ) than females (EOE c2 $=4 \%, 95 \%$ CI: $2-$ $4 \%$; depression $\mathrm{c} 2=14 \%, 95 \% \mathrm{CI}: 14-15 \%)$. Unique environmental factors explained the majority of variance in EOE and depression in males and females. This is the first twin study to examine EOE and depression in a non-Western population. Our results suggested sex differences in the aetiology of EOE and depression. Behavioural intervention for comorbid depression and obesity might be a useful avenue to explore in males. Our findings differ from results reported from Western population, highlighting the importance of cross-cultural comparisons in behavioural genetic research.

\section{Investigating intergenerational associations: revisiting the children-of-twins design I}

Tom McAdams, King's College London, United Kingdom; Eivind Ystrom, Norwegian Institute of Public Health, Norway; Line C. Gjerde, Norwegian Institute of Public Health, Norway; Espen Eilertsen, Norwegian Institute of Public Health, Norway; Fruhling Rijsdijk, King's College London, MRC Social, Genetic and Developmental Psychiatry Centre, Institute of Psychiatry, Psychology and Neuroscience, London, UK, United Kingdom

Datasets comprising twins and their children can be a useful tool for those interested in understanding the nature of intergenerational associations between parent and offspring phenotypes. For example, by using such data it is possible to ask whether parent-child associations can be explained via genetic transmission, and/or whether associations remain significant after accounting for genetic relatedness between parent and child. By applying structural equation models (SEMs) to children-of-twins (CoT) data, it is possible to quantify the relative importance of genetic versus non-genetic (social) pathways. In this talk I will review the SEMs that have been used to analyse CoT data to date. I will highlight some of the limitations that these models have, and discuss some limitations of the CoT datasets that these models have previously been applied to. For example, previous CoT models have had low power to parameterise the aetiology of the offspring phenotype, leading to questions about the models ability to identify the nature of intergenerational transmission. It is also not possible to detect offspring shared environmental effects using cousin data. I will present new variants of the CoT model, showing that including multiple offspring per parent solves many of the shortcomings of previous CoT models, dramatically increases power, and makes it possible to ask questions about the direction of effects between parent and child using a single CoT dataset (i.e. parent-to-child versus child-to-parent effects). I will demonstrate the power and utility of these models using simulated data and real data from the Intergenerational Transmission of Risk Project (a subsample of the Norwegian Mother and Child Cohort Study). I will finish by highlighting further planned model developments including multivariate and longitudinal CoT models.

\section{GWAS of educational attainment: phase 3 - main results}

Aysu Okbay, Vrije Universiteit Amsterdam, Netherlands; Robbee Wedow, University of Colorado Boulder, United States; Edward Kong, Harvard University, United States; Patrick Turley, Broad Institute of MIT and Harvard, United States; James Lee, University of Minnesota, Twin Cities, United States; Meghan Zacher, Harvard University, United States; Kevin Thom, New York University, United 
States; Anh Tuan Nguyen, Viet University of Southern California, United States; Omeed Maghzian, Harvard University, NBER, United States; Richard Karlsson Linnér, Vrije Universiteit Amsterdam, Netherlands; Matthew Robinson, The University of Queensland, Australia; Social Science Genetic Association Consortium, United States; Peter Visscher, The University of Queensland,Australia; Daniel Benjamin, University of Southern California,United States; David Cesarini, New York University, United States

Genetic factors are estimated to account for at least $20 \%$ of the variation across individuals for educational attainment (Rietveld et al., 2013). The results of the latest GWAS for educational attainment identified 74 genome-wide significant loci for educational attainment (Okbay et al., 2016). Here, in one of the largest GWAS to date, we increase our sample to nearly 750,000 individuals, and we identify over 600 genome-wide significant loci associated with the number of years of schooling completed. Note that at the time of writing, we will likely have updated our meta-analysis to include over $1,000,000$ individuals. In this presentation, I will present the details of this new meta-analysis, including analyses on the $\mathrm{X}$ chromosome. I will also present details of our quality control procedures, a thorough investigation of population stratification biases in our results, and novel approaches to identifying variants associated with theoretically related phenotypes like late onset Alzheimer's disease or cognitive function, using a new approach called Multi-Trait Analysis of GWAS (Turley et al., 2017). I will conclude by highlighting some basic findings from our polygenic prediction section and discussing the relevance of our findings for behavioral genetics and social sciences.

\section{Intergenerational transmission of adult to child ADHD in a population-based cohort study}

Eivind Ystrom, Norwegian Institute of Public Health, Norway; Espen Eilertsen, Norwegian Institute of Public Health, Norway; Fruhling Rijsdijk, King's College London, MRC Social, Genetic and Developmental Psychiatry Centre, Institute of Psychiatry, Psychology and Neuroscience, London, UK, United Kingdom; Line C. Gjerde, Norwegian Institute of Public Health, Norway; Tom McAdams, King's College London, United Kingdom

Attention deficit hyperactivity disorder (ADHD) is characterized by a persistent pattern of inattention and/or hyperactivity-impulsivity that interferes with functioning. Previous twin and family studies have shown moderate estimates of heritability for adult ADHD and high estimates of heritability of child ADHD. Across these studies, there has been little evidence of substantial effects of environmental factors shared by siblings. However, there is to date comparatively little knowledge on to what extent adult and child ADHD have common genetic risk factors. By using the Intergenerational Transmission of Risk (IToR) study, a twin-family subsample of the Norwegian Mother and Child Cohort study, we will estimate: (1) heritability of adult ADHD, (2) heritability of child ADHD, (3) genetic transmission of adult ADHD to child ADHD, (4) genetic factors specific to child ADHD, and (5) possible direct effects of parental ADHD to child ADHD. To estimate such parameters, we will apply an extended children of twin model on the IToR dataset comprising a large number of sibling/twin families. The results will inform on the biological underpinnings of the similar phenomena of adult and child ADHD. Our estimates of genetic innovation across age is of importance to molecular genetic studies where different age groups are often used in a single sample.

\section{Children's genetic risk for obesity evokes parental feeding behavior: evidence for gene-environment correlation}

Saskia Selzam, King's College London, United Kingdom; Tom McAdams, King's College London, United Kingdom; Robert Plomin, King's College London, Institute of Psychiatry, Psychology \& Neuroscience, United Kingdom; Clare Llewellyn, University College London, United Kingdom

Background: Parental feeding behaviors (restriction pressure) are widely assumed to have a causal influence on a child's weight; however, interest has grown in the idea that the child's weight may also 'elicit' feeding behaviors in the parents. Longitudinal data support this interpretation but cannot definitely identify causation. A novel approach is to examine associations between the child's genetic risk for obesity and parental feeding behaviors (gene-environment correlation).

Methods: BMI genome-wide polygenic scores (GPS) were created for 6710 unrelated children from the Twins Early Development Study using genome-wide association study summary statistics from an independent meta-analysis. Parental 'pressure' and 'restriction' were assessed using the Child Feeding Questionnaire when children were 10 years old. Child BMI standard deviation scores (BMI SDS) were calculated from parent-reported height and weight at 10 years. Correlations and polynomial contrasts tested associations between the GPS and parental feeding behaviors. In addition, we performed Genome-wide Complex Trait Analysis (GCTA) and twin analyses to calculate both narrow and broad-sense heritability estimates, as well as genetic correlations between BMI SDS and parental feeding styles. Results: As expected, the GPS was correlated with BMI SDS $(r=.192$, $\left.\mathrm{p}=1.96 \times 10^{34}\right)$. Consistent with the gene-environment correlation hypothesis, child BMI GPS was significantly positively associated with parental 'restriction' $\left(\mathrm{r}=.076, \mathrm{p}=6.52 \times 10^{7}\right)$ and negatively associated with 'pressure' $\left(r=-0.079, p=2.80 \times 10^{7}\right)$. Effects were linear across the GPS distribution. Our results remained unchanged after controlling for parental SES and parental weight. SNP-heritability estimates for parental feeding behaviors were negligible and non-significant, whereas twin-heritability estimates were moderate for parental 'pressure' and 'restriction'. Genetic correlations based on the twin model were moderate and negative between BMI SDS and 'pressure' and moderate and positive between BMI SDS and parental 'restriction'.

Conclusions: Results suggest an evocative gene-environment correlation, whereby heritable characteristics in the child 'elicit' parental behavior. These findings suggest that part of the observed association between children's BMI and parental feeding style is due to parents responding to genetically determined characteristics of their child.

\section{Phenotype harmonization: challenges and achievements within a large international consortium}

Gitta Lubke, University of Notre Dame, United States; Justin Luningham, University of Notre Dame, United States; Dorret Boomsma, Vrije Universiteit Amsterdam, Netherlands

Large consortia collaborating on gene-finding studies are faced with the reality that cohorts may differ with respect to questionnaires that were collected, raters, and age of the subjects at which the data were obtained. Given the need to optimize total sample size and power, a 
practical strategy is to use whatever phenotype data are available and proceed with GWASs with or without corrections for differences in data characteristics. The increase in power due to larger sample size can be counterbalanced however by a loss induced by phenotypic heterogeneity. Psychometric modeling approaches aimed at phenotype harmonization can alleviate to some extent this loss of power, but require item level questionnaire data from all partners. Missing data patterns due to lack of overlap in phenotype items across partners are a major problem in phenotype harmonization approaches. In the ACTION consortium, partners agreed to upload item level phenotype data in a pre-structured format in $\mathrm{R}$ such that item level data can be easily combined across partners. Using the same format permits not only an overview of the available data but also easy selection of items, raters, age groups, etc., for any type of analysis with simple R scripts. Importantly, one of the ACTION partners (NTR) has collected a phenotype reference panel consisting of all aggression-related items collected by ACTION partners. Current simulations tailored to the data available in ACTION show that utilizing such a reference set during harmonization increases power in subsequent GWASs. Collecting phenotypic reference panel data in consortia therefore seems to be rather desirable from the perspective of a cost/benefit trade-off.

\section{GWAS of educational attainment, phase 3: prediction}

Robbee Wedow, University of Colorado Boulder, United States; Aysu Okbay, Vrije Universiteit Amsterdam, Netherlands; Edward Kong, Harvard University, United States; Patrick Turley, Massachusetts General Hospital; Broad Institute of MIT and Harvard, United States; James Lee, University of Minnesota, Twin Cities, United States; Meghan Zacher, Harvard University, United States; Kevin Thom, New York University, United States; Anh Tuan Nguyen, Viet University of Southern California, United States; Omeed Maghzian Harvard University, United States; Richard Karlsson Linnér, Vrije Universiteit Amsterdam, Netherlands; Matthew Robinson, The University of Queensland, Australia; Social Science Genetic Association Consortium, United States; Peter Visscher, The University of Queensland, Australia; David Cesarini, New York University, United States; Daniel Benjamin, University of Southern California, United States

Genetic factors are estimated to account for at least $20 \%$ of the variation across individuals for educational attainment (Rietveld et al., 2013). The results of the latest GWAS for educational attainment identified 74 genome-wide significant loci for educational attainment (Okbay et al., 2016). Here, in one of the largest GWAS to date, we increase our sample to nearly 750,000 individuals, and we identify over 600 genome-wide significant loci associated with the number of years of schooling completed. Note that at the time of writing, we will likely have updated our meta-analysis to include over $1,000,000$ individuals. In this presentation, I will highlight our polygenic prediction results. We predict using 2 samples, the Health and Retirement Study (HRS) and The National Longitudinal Study of Adolescent to Adult Health (Add Health). Overall, we find that our polygenic scores explain around $10 \%$ of the variation in educational attainment, making these scores some of the most powerful genetic predictors created to date. I will also focus on the prediction of other related phenotypes, such as high school and college completion, verbal cognition, GPA, etc., and I will contrast the predictive power of our scores at different $\mathrm{p}$-value thresholds and at different sample sizes of our meta-analysis. Finally, I will present a novel analysis demonstrating how the predictive power of our polygenic score fluctuates as a result of changes in the heritability and genetic correlation of educational attainment across the cohorts included in our study.

\section{References}

Okbay, A., Beauchamp, J. P., Fontana, M. A., Lee, J. J., Pers, T. H., Rietveld, C. A., ... Benjamin, D. J. (2016). Genome-wide association study identifies 74 loci associated with educational attainment. Nature, 533, 539-542. doi:10.1038/nature.

Rietveld, C. A., Medland, S. E., Derringer, J., Yang, J., Esko, T., Martin, N. W., ... Koellinger, P. D. (2013). GWAS of 126,559 individuals identifies genetic variants associated with educational attainment. Science, 340(6139), 1467-1471. doi:10.1126/scienc.

\section{Large scale genome wide association analyses of food intake uncover associations with health and behavior}

Fleur Meddens, Vrije Universiteit, Amsterdam, Complex Trait Genetics, Center for Neuroscience and Cognitive Research, Netherlands; Ronald de Vlaming, VU, Netherlands; Niels Rietveld, Erasmus University, Rotterdam, Netherlands; James Lee, University of Minnesota, Twin Cities United States; Daniel Benjamin, University of Southern California, United States; Carson Chow, NIH/NIDDK, United States; Philipp Koellinger, Vrije University, Netherlands

The molecular genetics of food intake knows two substantial challenges. First, only two genetic loci have been discovered in the published GWAS literature. Second, there exist no reliable measurement methods of habitual food intake in the first place: systematic underreporting of total food intake is the norm, and especially problematic because it is associated with body mass index-potentially giving rise to spurious associations with obesity and food intake.

We propose a new method for processing self-report food intake data that largely eliminates the association with systematic misreporting bias of total food intake. Our measure of food intake is determined by relative intakes of protein, carbohydrate, fat, and sugar intake, which we subject to genome wide association analyses. The current preliminary sample size is $\mathrm{N} \approx 90,000$, which is projected to more than double in size in the near future. Here, we present our preliminary findings. These findings give rise to new insights into the biological basis of food preferences, and quasi-causal associations between food intake and health outcomes.

\section{Genetic and environmental risk factors for DSM-5 section II personality disorders and section III pathological personality traits}

Ted Reichborn-Kjennerud, Norwegian Institute of Public Health, Norway; Robert Krueger, University of Minnesota, United States; Eivind Ystrom, Norwegian Institute of Public Health, Norway; Fartein Ask Torvik, Norwegian Institute of Public Health, Norway; Tom Rosenström, Norwegian Institute of Public Health, Norway; Steven Aggen, Virginia Commonwealth University, United States; Susan South, Purdue University, United States; Michael Neale, Virginia Commonwealth University, United States; GunPeggy Knudsen, Norwegian Institute of Public Health, Norway; Kenneth Kendler, Virginia Commonwealth University, United States; Nikolai Czajkowski, University of Oslo, Norway

Background: The classification of personality disorders (PDs) in DSM-5 Section II includes 10 categorical PDs. The Alternative Model (Section III) includes criteria for dimensional measures of pathological personality traits organized into five domains. The degree to which genetic and environmental influences on the two conceptualizations are shared is not known. 
Methods: We used data from a large longitudinal population-based study of young adult Norwegian twins. DSM-IV PDs were assessed using structured interviews. The five domains of the DSM-5 Alternative Model plus a domain explicitly targeting compulsivity were measured by a short self-report inventory. Schizotypal, Paranoid, Antisocial, Borderline, Avoidant and Obsessive-compulsive PDs were assessed at the same time as the pathological personality traits and 10 years previously. Schizoid, Histrionic, Narcissistic, and Dependent PDs were only assessed at the first interview. Biometric models were used to estimate overlap in genetic and environmental risk factors.

Results Genetic correlations between the individual domains and PDs ranged from +0.21 to +0.91 . When measured concurrently, there was $100 \%$ overlap between genetic risk factors for the pathological trait domains in aggregate and for Paranoid, Schizotypal, Antisocial, Borderline and Avoidant PDs respectively.

Conclusion: The pathological personality trait domains of the Alternative Model for classification of PDs in DSM-5 section III, appears to tap, at an aggregate level, the same genetic risk factors as the DSM5 section II classification for most of the PDs.

\section{Childhood social class as a moderator of late-life cognitive abilities}

Malin Ericsson, Karolinski Institute, Sweden; Ralf Kuja-Halkola, Karolinska Institutet, Sweden; Catalina Zavala, University of Southern California, United States; Chandra Reynolds, University of California, Riverside, United States; Nancy L. Pedersen, Karolinska Institutet, Sweden

Introduction: Genetic influences on cognitive ability vary across socioeconomic groups, where the heritability of cognitive ability is attenuated in poor childhood environments compared to more advantaged environments where the genetic influences seems to have a greater influence [1]. The objective of this study was to investigate this moderation by socioeconomic environment on cognitive ability persist into late life. We investigated if increase in genetic variance with increasing social class is maintained in advancing age, as the distance from childhood and education increases.

Methods: We used data from the Swedish Adoption/Twin Study of Aging (SATSA), consisting of 859 individuals (Twins Reared Apart= 425 (200 pairs) and Twins Reared Together $=434$ (220 pairs)) aged 50-96, with cognitive data from up to 9 in-person testing occasions spanning over 28 years. We utilized data from all time points using the intercept at the centering age 65 . Cognitive ability was measured via a principal component score that covered four domains: verbal, fluid, memory and perceptual speed abilities. A general ability score was derived through principal component analysis. Childhood social class (parental occupation) and education were self-reported. Models with childhood social class or education moderating A, C, and E for cognitive ability were applied to both the total sample and separately to the TRT.

Results: The results from the moderator models for both childhood social class and own education showed similar patterns. $\mathrm{C}$ increased with higher socioeconomic measures at the same time as a relative decrease in A for general ability, verbal and spatial. Moderation by childhood social class was statistically significant only for verbal abilities and by education for general ability and verbal abilities. Moderation by childhood social class differs between childhood and late-life cognitive abilities. We will be replicating these findings on other samples within IGEMS.

\section{References}

1. Turkheimer, E., et al. Socioeconomic status modifies heritability of IQ in young children. Psychol Sci, 2003. 14(6): p. 623-8.

\section{Preferring domination or egalitarianism: genetic and environmental structure of social dominance orientation}

Thomas H. Kleppestø, University of Oslo, Norway; Nikolai Czajkowski, University of Oslo, Norway; Olav Vassend, University of Oslo, Norway; Espen Røysamb, University of Oslo, Norway; Nikolai Haahjem Eftedal, University of Oslo, Norway; Jonas R. Kunst, University of Oslo, Norway; Lotte Thomsen, University of Oslo, Norway

Purpose: Social Dominance Orientation (SDO) is a measure that describes individual differences in the preference for some groups to dominate over others. SDO is a robust predictor of sociopolitical attitudes and behaviors, including racism and extreme intergroup violence. Initially, SDO was considered a unitary construct, but more recently it has been argued that the construct consists of two subcomponents: Dominance (SDO-D) and Egalitarianism (SDO-E). Dominance is the desire for some groups to be actively oppressed (e. g. old-fashioned racism), while SDO-E is a preference for inequality without any support for overt oppression of groups. This paper seeks to understand the genetic and environmental structure of Dominance and Egalitarianism.

Methods: Our sample consists of male monozygotic $(\mathrm{N}=239)$, female monozygotic $(\mathrm{N}=304)$, and male dizygotic $(\mathrm{N}=324)$ and female dizygotic $(\mathrm{N}=412)$ twins. We measured SDO with a Norwegian translation of SDO-6. Model parameters were estimated with full information maximum likelihood using the R package OpenMx.

Results: An AE model without sex limitation was found to have overall best fit compared to seven other models, as evaluated by lowest AIC (3518.6). The estimated heritabilities for Dominance and Egalitarianism were found to be 0.36 and 0.24 respectively, while the genetic correlation between the SDO subdomains was estimated to 0.5 (CI: 0.294-0.686). Conversely, the unique environmental correlation was found to be 0.38 (CI: $0.297-0.456$ ).

Conclusion: SDO-Dominance and SDO-Egalitarianism modestly overlap at the underlying genetic and environmental architecture. Our results suggest no evidence for shared environmental effects, nor any quantitative sex differences.

\section{Social stress, epigenetic changes and pain}

Mina Eriksen, The National Institute of Occupational Health, Norway; Daniel Pitz Jacobsen, The National Institute of Occupational Health, Norway; Johannes Gjerstad, The National Institute of Occupational Health, Norway

Aims: Bullying is a prevalent issue in society, with adverse effects ranging from psychological symptoms to somatic ailments like chronic pain. The purpose of this study was to generate new knowledge about the underlying mechanisms behind this association. Using an animal model, we investigated the changes in microRNA expression in plasma, in the pituitary gland and the adrenal gland following social stress. 
Methods: A resident-intruder paradigm where male Sprague Dawley rats (intruders) were exposed to male Long Evans rats (resident) one hour daily for a week, was used. Bodyweight was measured and blood samples were collected throughout the experiment. Changes in plasma microRNA expression was determined by qPCR.

Results: Rats exposed to social stress showed reduced weight gain compared to controls. Preliminary results suggested that social stress increased the plasma expression of miR-146a-5p, miR-30c-5p and miR-223-3p.

Conclusions: The data showed that social stress gives reduced weight gain and increase changes in the expression of several circulating microRNAs. How this affects the development of persistent pain remains to be investigated.

\section{Using a three-item questionnaire to determine zygosity in Chinese young twins: a validation study}

Mo Zheng, The University of Hong Kong; Connie Suk-Han Ho, The University of Hong Kong, Hong Kong; Catherine Mcbride, Chinese University of Hong Kong, Hong Kong; Bonnie W. Y. Chow, City University of Hong Kong, Hong Kong; Simpson W. L. Wong, Education University of Hong Kong, Hong Kong; Richard K. W. Choy, Chinese University of Hong Kong, Hong Kong; Mary M. Waye, Chinese University of Hong Kong, Hong Kong; Richard Olson, University of Colorado Boulder, United States

Using questionnaire method to determine zygosity is common in twin research due to its low cost and time saving advantage. A recent studysup1/sup by the authors show that a simple 3-item parent questionnaire can achieve accuracy of over $90 \%$ in zygosity classification in a Chinese school-aged twin sample. The three questions asked (1) parental perception of their twins' zgyosity, (2) twins' resemblance and (3) whether twins were confused by strangers. The current study attempted to replicate the effectiveness of this 3-item questionnaire in an independent validation sample. Three methods were employed to evaluate parental report accuracy, namely summed score, logistic regression, and decision tree. All three methods yielded similarly high classification accuracy in both the original sample and the validation sample. Furthermore, the current study explored the possibility to improve the questionnaire by adding additional physical characteristics items, such as twins' similarity in hair texture. How parental responses were affected by the advice of medical professionals and information about the placenta was also discussed.

\section{References}

1. Ho, C. S. H., Zheng, M., Chow, B. W. Y., Wong, S. W.Lim, C. K., \& Waye, M. M. (2017). Adequacy of using a three-item questionnaire to determine zygosity in Chinese young twins. Behavior Genetics, 47(2), 244-254.

\section{A twin study of normative personality and DSM-IV personality disorder criterion counts; evidence for separate genetic influences}

Nikolai Czajkowski, University of Oslo, Norway; Steven Aggen, Virginia Commonwealth University, United States; Robert Krueger, University of Minnesota, United States; Kenneth Kendler, Virginia Commonwealth University, United States; Michael Neale, Virginia Commonwealth University, United States; GunPeggy Knudsen, Norwegian Institute of Public Health, Norway; Nathan Gillespie, Virginia Commonwealth University, United States; Espen Røysamb,
University of Oslo, Norway; Kristian Tambs, Norwegian Institute of Public Health, Norway; Ted Reichborn-Kjennerud, Norwegian Institute of Public Health, Norway

Background: Normative personality and DSM-IV personality disorders (PD) have both been found to be heritable, but the extent to which they share genetic influences is largely unexplored. Methods: 2,282 Norwegian twins were assessed for DSM-IV criteria for personality disorders using structured interviews. Ten years later years the twins were re-interviewed, and normal personality was assessed using the Big Five Inventory. The amount of genetic variance that DSM-IV PD criterion counts had in common with the domains of the Big 5 was estimated by means of multivariate Cholesky twin decompositions.

Results: While the heritability of the number endorsed criteria for the personality disorders ranged from 0.2 to 0.41 , the proportion of genetic variance that was unique to each PD ranged from $19 \%$ for Avoidant to $79 \%$ for Schizotypal (median of 58\%). Conversely, the proportion of environmental variance unique to the personality disorder criteria ranged from $79 \%$ to 99 (median 97\%).

Conclusion: A considerable proportion of the genetic variance and virtually all environmental variance is unique to the personality disorders, and not shared with the five broad domains of normal personality.

\section{The etiology of stability and change in social withdrawal through childhood: a genetically informative study}

Geneviève Morneau-Vaillancourt, Université Laval, Canada; Alexandra Matte-Landry, Université Laval, Canada; Ginette Dionne, Université Laval, Canada; Mara Brendgen, Université du Québec à Montréal, Canada; Frank Vitaro, Université de Montréal, Canada; Richard Ernest Temblay, Université de Montréal, Canada; Michel Boivin, Université Laval, Canada

Background: Social withdrawal in childhood has been associated with a host of socio-emotional difficulties later on (Boivin, Petitclerc, Feng, \& Barker, 2010; Rubin \& Coplan, 2004). Yet, significant gaps remain in our knowledge of its development, notably the etiology of individual differences in social withdrawal in childhood. The current study investigates the genetic and environmental etiology of stability and change in social withdrawal within a longitudinal perspective.

Methods: A number of 798 twins were assessed by their teacher for social withdrawal at 5 time-points from kindergarten to grade 6 . We tested a linear latent growth curve model to evaluate intra-individual stability and change in social withdrawal from kindergarten to grade 6 . We assessed the contribution of genetic, shared and non-shared environmental factors to the initial level and to the rate of change in social withdrawal with a biometric latent growth curve model.

Results: No significant intra-individual change was observed from kindergarten through grade 6 , meaning that overall, levels of social withdrawal where stable through time. Inter-individual differences were found for the intercept factor, meaning that there was significant variation in social withdrawal scores at kindergarten. These differences were explained by genetic and non-shared environmental factors. Finally, results show that non-shared environmental factors explain most of individual differences in social withdrawal at every time point. A significant proportion of the time-specific variance was also explained by genetic factors.

Conclusions: The stability in the average trajectory of social withdrawal through childhood could be explained by the lack of change in this phenotype in childhood, but it could also be a result of averaging different trajectory directions (e.g., increasing or decreasing slopes). 
Further research could shed light on the genetic and environmental mechanisms or interactions potentially underlying the etiology of social withdrawal.

\section{References}

Boivin, M., Petitclerc, A., Feng, B. \& Barker, E. D. (2010). The developmental trajectories of peer victimization in middle to late childhood and the changing nature of their behavioral correlates. Merrill-Palmer Quarterly, 56, 231-260. doi:10.1353/mpq.0.0050.

Rubin, K. \&; Coplan, R. (2004). Paying attention to and not neglecting social withdrawal and social isolation. Merrill-Palmer Quarterly, 50, 506-534. doi:10.1353/mpq.2004.0036.

\section{Disentangling genetic transmission from the environmental effect of maternal depression during pregnancy on offspring ADHD}

Espen Moen Eilertsen Norwegian, Institute of Public Health, Norway; Line C. Gjerde, Norwegian Institute of Public Health, Norway; Fruhling Rijsdijk, King's College London, MRC Social, Genetic and Developmental Psychiatry Centre, Institute of Psychiatry, Psychology and Neuroscience, London, UK, United Kingdom; Tom McAdams, King's College London, United Kingdom; Eivind Ystrom, Norwegian Institute of Public Health, Norway

Maternal depression during pregnancy has been associated with ADHD in the offspring. This association may represent a causal effect, where inutero exposure has detrimental consequences for offspring development. However, because the association in general has not been studied with sufficient control for extraneous variables, causal inferences are not justified. Because mothers and their offspring are genetically related, and typically share many aspects of the environment, both pleiotropy and environmental variables not accounted for could be completely, or partially, responsible for the observed association.

The aim of the current study is to expand current knowledge by disentangling genetic transmission from the environmental effect of maternal depression during pregnancy on offspring ADHD. We approach the problem using and extended children-of-twins design, utilizing differential genetic relatedness among sibling mothers and their offspring in order to separate these alternative models for mother-offspring resemblance. Specifically, we will use structural equation models that allows us to quantify and test, genetic and environmental pathways from mother to offspring.

The study is part of the Intergenerational Transmission of Risk Project, using data from The Norwegian Mother and Child Cohort Study, a large prospective population-based study of parents and offspring in Norway. Results will be presented at the Conference.

\section{The promise of pooled twin studies for exploring genetic and epigenetic interplay: IGEMS}

Nancy L. Pedersen, Karolinska Institutet, Sweden; IGEMS Consortium, University of Southern California, United States

The Interplay of Genes and Environment across Multiple Studies (IGEMS) consortium of 15 existing longitudinal twin and family studies was initiated with a central focus on determining gene-environment interplay of social context and mid- and late-life functioning. This symposium presents a variety of analyses that demonstrate the promise of pooled twin studies for exploring genetic and epigenetic interplay. We provide examples of how behavioral genetic methods can be used for understanding social class effects, age and sex moderation of GE on a harmonized measure of financial strain and childhood social class as a moderator of late-life cognitive abilities. We also demonstrate how PRS scores for well-being are predict health problems, and evaluate longitudinal variance components across 10 years for the epigenetic clock and its constituent CpGs.

Supported by Grant No. R01 AG037985.

\section{Genome-wide association study of executive functions and their genetic relationship with IQ, academic achievement and psychopathology}

Georgina Donati, Birkbeck, United Kingdom; Iroise Dumontheil, Birkbeck College, University of London, United Kingdom; Emma Meaburn, Birkbeck College, University of London, United Kingdom

Impaired executive function (EF) is associated with increased risk of psychopathology and lower academic achievement. However, relatively little is known about the genetic architecture of EF traits, and the pathways linking genes, cognitive processes and later academic and mental health outcomes. We previously characterized three latent cognitive variables in an adolescent sample; two EF traits (working memory and inhibitory control) and processing speed. We found that each trait explained differing unique variance in maths, English and science as well as DSM V assessments, suggesting that in order to understand specificity in academic and psychological outcomes, an understanding of individual differences at a neurocognitive level is necessary. The goal of the present study was to add to current understanding of the genetics of EF in adolescence and their genetic relationship to psychological and academic outcomes.

We performed univariate genome-wide association studies (GWAS) for working memory, inhibition and processing speed $(\mathrm{N}=$ 4,817) with adolescents from the Avon Longitudinal Study for Parents and Children. Linkage disequilibrium (LD) score regression was used to estimate the heritability of each trait as well as genetic correlations between our traits and measures of attainment, IQ and psychopathology.

A moderate SNP-based heritability estimate was found for WM (0.25) with a smaller estimate for processing speed and IC. Uniformly high (0.66-1.00) genetic correlations were observed for WM and intelligence and educational traits. Moderate to high correlations were found with Anorexia, Depressive Symptoms and ADHD.

The picture was more varied for processing speed, as with IC, possibly due to the large standard errors for their heritability estimates. Univariate GWA analyses failed to detect any genome-wide significant associations. However, 50 lead single nucleotide polymorphisms (SNPs) reached suggestive significance, 15 of which are located in genes previously implicated in GWA studies of education, cognition or psychopathology. MAGMA gene analysis showed there to be a higher representation of genes expressed in the brain for WM but not the other traits. The results suggest that WM is highly polygenic trait with links to risk for specific psychopathologies. However it was not clear that studying EF traits is more powerful that studying the end phenotypes themselves.

\section{The genetics of well-being}

Ragnhild Bang Nes, Norwegian Institute of Public Health, Norway; Meike Bartels, Vrije Universiteit Amsterdam, Netherlands; Bart Baselmans, Vrije Universiteit Amsterdam, Netherlands; Espen Røysamb, University of Oslo, Norway; Nikolai Czajkowski, University of Oslo, Norway 
Research into well-being and well-functioning has gained increasing interest across various disciplines in recent years. Within geneticallyinformative research, wellbeing is now studied with increasingly advanced multivariate and longitudinal designs as well as molecular genetic methods. This symposium features five different authors and presents the most recent findings from genetically-informative studies of well-being. The first presentation gives an overview of the main findings and explores the genetic and environmental commonality and specificity underpinning different components of well-being and illbeing. The second paper presents a genetically-informative approach, including molecular genetics, to elucidate one of the main discussions in the field of positive psychology, namely overlap and distinction between hedonic and eudaimonic well-being. The third presentation features two multivariate genome-wide meta-analyses (multivariate GWAMA's) of life satisfaction, positive affect, neuroticism, and depressive symptoms. The fourth paper presents findings from a longitudinal twin study exploring phenotypic and genotypic associations between anxiety, depression and life satisfaction after midlife. The last presents a discordant MZ twin analysis on associations between a number of somatic diseases and overall satisfaction with life.

\section{Longitudinal associations between anxiety and depressive symptoms during childhood: a genetically informed cross-lagged study}

Laurence Tanguay-Garneau, Université Laval, Canada; Michel Boivin, Université Laval, Canada; Bei Feng,Université Laval Canada; Alexandra Matte-landry, Université Laval, Canada; Mara Brendgen, Université du Québec à Montréal, Canada; Frank Vitaro, Université de Montréal, Canada; Ginette Dionne, Université Laval, Canada

Background: Anxiety symptoms (AS) and depressive symptoms (DS) are associated during childhood, which could be accounted for by dynamic temporal interactions between the two sets of symptoms, as well as by shared etiological features (Garber \& Weersing, 2010). The present study aimed to document the longitudinal contributions between AS and DS by taking into account etiological factors in a normative sample of twins over the course of elementary school.

Methods: 1112 twins were assessed by their teacher for AS and DS at 5 time-points from kindergarten to grade 6 . A cross-lagged structural model was estimated to examine the cross-sectional associations as well as cross-lagged contributions between AS and DS. The etiology of the associations between AS and DS within and across ages was examined by fitting cross-lagged twin models (Burt, McGue, Krueger, \& Iacono, 2005).

Results: Most of the variance in AS (90.4-96.6\%) and DS (87.2$94.5 \%)$ was time-specific and more genetic in nature for DS $\left(\mathrm{a}^{2}=\right.$ $\left.33 \% / 33.2-50.5 \% / 52.5 \% ; \mathrm{e}^{2}=44 \% / 44.6-57 \% / 56.2 \%\right)$ than AS $\left(\mathrm{a}^{2}=\right.$ $\left.21.7-35.5 \% / 35.9 \% ; \mathrm{e}^{2}=68.7-70.2 \% / 70.9 \%\right)$. Previous DS (cross-age) best accounted for subsequent DS than cross-lag or previous common effects through both genetic and unique environmental influences, whereas AS from Kindergarten to Grade 3 was best accounted for by cross-lags from previous DS than cross-age or previous common effects through both common genetic and unique environment influences. Thereafter, previous AS contributed more to subsequent AS than cross-lag or previous common effects mainly through genetic influences.

Conclusions: DS in the early school years appear central to the development of both AS and DS through cross-lag and cross-age genetic and unique environmental influences.

\section{References}

Burt, S. A., McGue, M., Krueger, R. F., \& Iacono, W. G. (2005). How are parent-child conflict and childhood externalizing symptoms related over time? Results from a genetically informative cross-lagged study. Dev Psychopathol, 17(1), 145-165. doi:Ć10.1017/ S095457940505008X.

Garber, J., \& Weersing, V. R. (2010). Comorbidity of Anxiety and Depression in Youth: Implications for Treatment and Prevention. Clinical Psychology: Science and Practice, 17(4), 293-306. doi: Ć10.1111/j.1468-2850.2010.01221.x.

\section{Heritability of non-medical use of prescription drugs among a sample of undergraduate students}

Cassie Overstreet, Virginia Commonwealth University, United States; Mackenzie Lind, Virginia Commonwealth University, United States; Elizabeth Do, Virginia Commonwealth University, United States; Kenneth Kendler, Virginia Commonwealth University, United States; Danielle Dick, Virginia Commonwealth University, United States; Ananda Amstadter, Virginia Commonwealth University, United States

Nonmedical use of prescription drugs (NMUPD) has been associated with a number of negative psychological and physical outcomes (e.g., depression, anxiety [Cai et al., 2010], ER visits [Warner et al., 2009], and overdose deaths, [Becker et al., 2008]). Given its increasing prevalence and associated consequences, NMUPD is a growing public health concern and significant economic burden (Birnbaum et al., 2011; Oderda et al., 2015). Despite the high prevalence, the environmental and genetic contributions to NMUPD remain understudied. Heritability estimates from studies of families and twins suggest moderate heritability; yet, NMUPD is often clustered with other forms of illicit drug use (e.g., heroin) in many analyses. Further, there are no estimates of NMUPD heritability from molecular data. Geneidentification efforts are in their nascent stage. Thus, the present study aimed to utilize molecular genetic methods to understand genetic contributions to NMUPD in a large sample of undergraduate students $(\mathrm{N}=7,603$, Mage $=18.53, \mathrm{SD}=.65 ; 61.1 \%$ female $)$. Lifetime NMUPD, reported by $15.6 \%$ of participants, was used as a binary variable in the present analyses and sex was included as a covariate. Preliminary results demonstrated an aggregate SNP heritability estimate of $4.7 \%$ $(\mathrm{SE}=.13)$ among participants of European ancestry $(n=2,941)$; however, the estimate did not significantly differ from zero. Genome-wide Complex Trait Analyses (GCTA) in the second largest sub-population, those of African ancestry, did not yield evidence of SNP-based heritability. GWAS are in progress to identify specific genetic variants associated with NMUPD and will also be presented. The present findings should be considered in light of the small sample size. Continued examination of the genetic architecture of NMUPD is important given the potential empirical (gene-findings efforts) and clinical (tailored prevention/intervention programs) implications.

\section{Anxiety, depression and life satisfaction after midlife. A twin study across 20 years}

Espen Røysamb, University of Oslo, Norway; Ragnhild Bang Nes, Norwegian Institute of Public Health, Norway; Nikolai Czajkowski, University of Oslo, Norway; Olav Vassend, University of Oslo, Norway 
Background: Life satisfaction is negatively associated with mental health problems such as anxiety and depression. There is limited knowledge about long term relations, and about the nature of the associations. The aim of this study was to (a) examine to what extent mental health problems around midlife predict level of life satisfaction 20 years later, (b) what role the personality trait of neuroticism plays in associations, and (c) how environmental and genetic factors contribute to relationships.

Methods: The Norwegian Twin Registry provided data for the study $(\mathrm{n}=1987)$. Twins born 1945-1960 participated in surveys around age 40 (time1) and age 60 (time2). Measures include subscales of the Symptom Check List (i.e. anxiety, depression) and Neuroticism from the Eysenck Personality Questionnaire, at time 1, and Satisfaction With Life Scale (SWLS) at time 2. Data were analyzed with multiple regression and biometric twin modelling including multivariate Cholesky models.

Results: Mean score on the SWLS at time 2 was 5.4 (s.d.=1.2; range 1-7), indicating a relatively high level of life satisfaction. Depression, but not anxiety, at time 1 predicted life satisfaction scores 20 years later $(\beta=-0.35)$, and the effect was partly, but not fully, accounted for by neuroticism. Biometric modelling showed that genetic factors played a major role in the associations between depression, neuroticism and later life satisfaction. However, most of the variance in life satisfaction was unrelated to previous symptoms and neuroticism, and was environmental rather than genetic in origin.

\section{Longitudinal variance components of the Horvath clock and its contributing CpGs}

Juulia Jylhävä, Karolinska Institute, Sweden; Chandra Reynolds, University of California, Riverside, United States; Jacob Hjelmborg, University of Southern, Denmark, Denmark; Elizabeth Munoz, University of California, Riverside, United States; Qihua Tan, University of Southern, Denmark, Denmark; Lene Christiansen, University of Southern, Denmark, Denmark; Sara Hägg, Karolinska Institutet, Sweden; Nancy L. Pedersen, Karolinska Institutet, Sweden

A measure of DNA methylation age, the Horvath's epigenetic clock, has recently emerged as a promising marker to predict mortality. However, the contribution of genetic and environmental factors to the epigenetic clock at different old ages is incompletely understood. Therefore, we estimated the magnitude of genetic and environmental influences to the epigenetic clock in 126 (52 MZs, 74 DZs) Swedish and Danish twin pairs at two time points: at mean age of 69 and 79 years. Using the Cholesky decomposition, we also estimated the magnitude of the sources for shared variation between the two ages. The DNA methylation data were assessed in whole blood using the Illumina $450 \mathrm{k}$ BeadChip and the epigenetic clock estimates were assessed using the Horvath algorithm. The analyses were adjusted for age, sex, and cell type heterogeneity. Intraclass correlations for MZ and DZs were 0.259 and 0.125 at 69 years and 0.282 and 0.020 at 79 years. The cross-time correlations were 0.341 and 0.294 for MZs and DZs. An AE model best explained the variation at both ages $(\mathrm{E}=$ $76.6 \%, \mathrm{~A}=23.4 \%$ at 69 years; $\mathrm{E}=78.1 \%, \mathrm{~A}=21.9 \%$ at 79 years). The cross-time covariation was primarily due to $\mathrm{A}$ than $\mathrm{E}(\mathrm{A}=69.7 \%, \mathrm{E}=$ $30.3 \%$ ). Individual $\mathrm{CpG}$ sites contributing to the Horvath's clock were also evaluated via bivariate Cholesky models within samples using M-values. Among the Swedish samples 53 pairs were selected for comparative analysis with the 43 pairs among the Danish samples. The average broad heritability across 317 sites in the Swedish samples was 19 and $12 \%$ at waves 1 and 2, respectively while for the Danish samples across 353 sites it was 24 and 19\%, respectively. Significant cross-wave associations were observed in $78 \%$ of the Danish but $29 \%$ of the $\mathrm{CpG}$ sites in the Swedish samples. Our results demonstrate that most of the variation in the epigenetic clock in old age is due to environmental factors and to a lesser extent due to genetics. However, the genetic sources of variation are largely the same across the old ages whereas those for the environmental ones seem to differ more.

\section{Genetic Instrumental Variable (GIV) regression: explaining socioeconomic and health outcomes in non- experimental data}

Casper Burik, Vrije Universiteit Amsterdam, Netherlands; Philipp Koellinger, Vrije University, Netherlands; Thomas DiPrete, Columbia University, United States

Estimating causal effects with non-experimental data is of central importance across multiple fields of scientific inquiry. Here, we propose genetic instrumental variables (GIV) regression for this purpose. GIV regression utilizes large-scale genome-wide association study (GWAS) results that now allow constructing predictive polygenic scores (PGS) for many human traits. Our approach is based on the idea that adding the true PGS for the outcome to a regression model would effectively eliminate bias arising from a genetic correlation between the outcome and an exposure of interest. Without the true PGS in the structural model, using genes as instrumental variables (IVs) as proposed in Mendelian Randomization (MR) is problematic due to pleiotropic effects of genes that invalidate IV regression. However, PGS capture only a small fraction of the heritability of most traits, partly because GWAS are estimated in finite sample sizes that yield noisy estimates of the effects of each genetic marker. We argue and empirically demonstrate that it is possible to correct attenuation bias by splitting the GWAS sample to obtain several PGS (i.e. multiple indicators) in the prediction sample that can be used as instruments for each other in IV regression. Then we extend the approach to the problem of estimating causal effects and gene-environment interactions with non-experimental data. Our approach produces reasonable estimates of the chip heritability of educational attainment (EA) and, unlike the results using MR, GIVbased estimates find that the positive correlation between body height and EA is primarily due to genetic effects.

\section{Associations between parent and child anxiety are bidirectional: evidence from mothers and fathers}

Yasmin Ahmadzadeh, King's College London, Institute of Psychiatry, Psychology \& Neuroscience, United Kingdom; Thalia Eley, King's College London, United Kingdom; Jenae Neiderhiser, The Pennsylvania State University, United States; Leslie Leve, University of Oregon, United States; Daniel Shaw, University of Pittsburgh, United States; David Reiss, Yale University, United States; Misaki Natsuaki, University of California, Riverside, United States; Tom McAdams, King's College London, United Kingdom

Anxiety symptoms in parents are associated with anxiety symptoms in offspring. However, little is known about the mechanisms that mediate this intergenerational transmission. Here we conducted the first genetically sensitive study to examine bidirectional effects between parent and child anxiety symptoms during middle childhood, using data from Cohort I of the Early Growth and Development Study $(n=346$ adoptive families). Child anxiety symptoms were measured by adoptive parent report on the Child Behavior Checklist and the Eley Anxiety Measure at ages 6-8 years. Adoptive mother and father anxiety symptoms were measured at corresponding time points using 
the State-Trait Anxiety Inventory. Birth mother's anxiety symptoms were included as a proxy for inherited risk, measured at child ages 5, 18 months and 4 years using the Beck Anxiety Inventory. Structural equation models were fitted to the data, with adoptive mothers and fathers assessed separately. Higher anxious symptoms in children at age seven predicted higher anxious symptoms in adoptive mothers at age eight. No child-to-father effects were observed. In contrast, higher anxious symptoms in adoptive fathers at age six predicted higher anxious symptoms in children at subsequent time points, while no mother-to-child effects were observed. The composite measure of birth mother anxiety was not associated with child anxiety, suggesting that the genetic factors associated with anxiety in adult parents are not involved in anxiety in young child offspring. The pattern of results was consistent for both measures of child anxiety. Overall, these results emphasise the importance of taking a family-wide perspective when researching the intergenerational transmission of anxiety. Additional analyses will now be conducted to examine the role of adoptive mothers and fathers within the same structural equation model, alongside a broader measure of birth parent internalising-to further examine the role of inherited risk within this sample.

\section{Genome-wide examination of common and rare variant associations with alcohol response in the UCSF family study}

Joseph Deak, University of Missouri, United States; Ian Gizer, University of Missouri, United States; Jacqueline Otto, University of Missouri, United States; Christopher Bizon, University of North Carolina, Chapel Hill, United States; Kirk Wilhelmsen, University of North Carolina, Chapel Hill, United States

Heritability estimates suggest that approximately $50 \%$ of the variance related to alcohol use disorder (AUD) can be attributed to genetic influences. Nonetheless, molecular genetic efforts to identify individual variants that contribute to AUD have generated mixed findings, partially attributable to AUD phenotypic heterogeneity. Specifically, if differences in symptom presentation or comorbid conditions among individuals result from separable risk mechanisms, including such phenotypic heterogeneity will reduce power to detect genetic influences underlying these distinct mechanisms. One approach for addressing this heterogeneity is to study an endophenotype focusing on a specific facet of AUD (e.g., level of response [LR] to alcohol). LR is the extent to which a specific dosage of alcohol produces the responses associated with alcohol consumption. Notably, previous molecular genetic studies of LR using the Self-Rating of the Effects of Alcohol (SRE) questionnaire have reported associations with multiple variants and genes of interest, but due to small sample sizes have yet to be replicated. The current study is an initial effort to examine the effects of genome-wide common and rare variation in relation to LR to alcohol by utilizing low-coverage whole genome sequence data in a subset of the UCSF Family Study $(\mathrm{N}=1,188)$ with both genotype and SRE data for their first five drinking episodes. A GWAS of common variation (MAF $>0.05$ ) was carried out using the software package EMMAX. Gene-based tests of rare variation $(\mathrm{MAF}<0.02)$ restricted to coding regions were conducted utilizing SKAT-O. The GWAS resulted in no variants reaching genome-wide significance $\left(\mathrm{p}=5.0 \times 10^{-8}\right)$. The top association was in an intergenic region of chromosome 3 p26.1 (rs112429089, p=5.69 $\times 10^{-8}$ ). Individual variants in multiple genes previously implicated with alcohol outcomes were nominally associated with SRE scores (e.g., TMEM132D, $\mathrm{p}=5.00 \times 10^{-6}$; CHRM2, $\mathrm{p}=2.08 \times 10^{-5}$ ). For the genebased analyses of rare variation, the top signal was an association with the SETX gene $\left(\mathrm{p}=2.08 \times 10^{-7}\right)$, with additional genes of interest being suggestively associated (e.g., ADCY4, $p=1.42 \times 10^{-4}$; BDNF, $p$ $=2.26 \times 10^{-4}$ ). These findings suggest that similar to AUD, molecular genetic studies of alcohol response will require larger samples to identify specific risk-conferring variants, as well as alternative modeling techniques for investigating rare variation occurring at lower population frequencies.

\section{The relationship between genes, impulsivity, and voter turnout}

\section{Christopher Dawes, New York, United States}

Recent research has shown that impulsivity is negatively related to political participation. Since the costs of engaging in politics are paid before the policy benefits are realized, impulsive individuals are theorized to be less likely to participate. In this study, we test whether genes associated with impulsivity predict voter turnout. To do so, we construct a polygenic score for impulsivity based on a genome-wide association study of the delay discount task. Further, we test whether impulsivity mediates any significant association between the polygenic score and voter turnout.

We conduct our analysis based on two US samples (Minnesota Twin and Family Study and the National Longitudinal Study of Adolescent Health). We find that the polygenic scores for impulsivity is significantly associated with some measures of voter turnout but not others. However, the highest relationship we observe is between the score and whether individuals register to vote in the first place. This finding is consistent with the fact that registering to vote tends to take more planning than voting.

\section{Gene $\times$ environment interplay on political participation: the effect of information demands}

\section{Christopher Dawes, New York, United States}

Empirically, one of the predictors of voter turnout is educational attainment. Education is theorized to influence voter turnout in part because it enables citizens to more easily overcome information demands associated with voting. The genetic propensity to acquire education, captured using a polygenic score, has also been shown to be ly related to voter turnout. In this study, we test whether the exogenous electoral environment, which dictates the information demands citizens face, moderates the relationship between the polygenic score for educational attainment and voter turnout. Every 10 years, the electoral district boundaries are redrawn in the United States in order to account for shifts in population. Previous research has found that this redistricting process tends to reduce electoral participation because it raises informational costs associated with voting for many citizens. This is because they are forced to choose among politicians they are unfamiliar with (rather than their familiar incumbent). We analyze the redistricting process in Minnesota following the 2010 census where the electoral boundaries were redrawn by a judicial panel. In our sample, $30 \%$ of registered voters had to choose between two non-incumbents running for the Minnesota House of Representatives in the 2012 election. We find a significant interaction between the polygenic score and the electoral environment; those at the upper end of the polygenic score were significantly more likely to vote when choosing among unfamiliar candidates than those at the lower end of the score. 


\section{Data integration for improved psychometric phenotypes in gene association tests}

Justin Luningham, University of Notre Dame, United States; Dan Mcartor, University of Notre Dame, United States; Meike Bartels, Vrije Universiteit Amsterdam, Netherlands; Dorret Boomsma, Vrije Universiteit Amsterdam, Netherlands; Gitta Lubke, University of Notre Dame, United States

Tests of genetic associations with psychological traits require substantial power due to the expected small effect sizes. Two proposed avenues for gaining power in genetic tests are increasing sample sizes and improving phenotypic measures (Robinson, Wray, Visscher, 2014). Common practice is to meta-analyse the results of gene-finding studies carried out across multiple cohorts through genetic consortia projects, thereby increasing sample-size. However, systematic differences in measurement and phenotype definition across cohorts may sacrifice the quality of the phenotypic measurement and reduce the power gain resulting from combining cohorts. We propose integrative data analysis (IDA) via psychometric measurement models for simultaneously increasing sample size and improving phenotype resolution. IDA for psychological phenotypes involves fitting a measurement model to pooled item-level data from all studies (Curran \& Hussong, 2009). We present a bi-factor model that isolates a common phenotype score among the full item set while separating out cohort-specific measurement differences. We investigate the utility of IDA for tests of genetic effects in pooled studies with different phenotype item content, reflecting the common situation of different questionnaires across consortia partners. A simulation study under a series of underlying phenotype measurement conditions demonstrates that the bi-factor model for IDA can increase power as much as $11 \%$, compared to meta-analysis using sum scores of truncated item subsets in each cohort. Power gains are greater when measurement differences exist across partners, as these differences are captured in the IDA model but not in the formation of sum scores. The benefits of IDA are maximized with the collection of a very small de novo sample with complete data, which we call a phenotypic reference panel. Implications for GWAS meta-analyses are discussed.

\section{Is the common variant rs10830963 in the melatonin receptor $1 \mathrm{~B}$ gene influencing the associations between depressive symptoms and glycemic traits?}

Kadri Haljas, University of Helsinki, Finland; Jari Lahti, University of Helsinki, Finland; Tiinamaija Tuomi, Finnish Institute for Molecular Medicine, University of Helsinki; Helsinki University Hospital; Folkhälsan Research Center, Finland; Bo Isomaa, Folkhälsan Research Center; Department of Social Services and Health Care, Jakobstad, Finland, Finland; Johan Eriksson, Folkhälsan Research Center; National Institute for Health and Welfare; Department of General Practice and Primary Health Care, University of Helsinki and Helsinki University Hospital Helsinki, Finland; Leif Groop, Diabetes and Endocrinology, Department of Clinical Sciences, Lund University Diabetes Centre, Malmö; Finnish Institute for Molecular Medicine, University of Helsinki, Finland; Katri Räikkönen, University of Helsinki, Finland

Background: Previous studies have identified a bidirectional association between depression and type 2 diabetes (T2D). It has been suggested that these disorders may share common underlying biological determinants. Because the neurohormone melatonin, which plays a key role in circadian regulation, has been implicated in both depression and T2D, we examined if a common variant rs 10830963 in the Melatonin Receptor 1B (MTNR1B) gene influenced the association between depressive symptoms and glycemic traits.

Methods: The Prevalence, Prediction and Prevention of Diabetes (PPP-Botnia) is a population-based study of 5,208 participants aged 18 to 78 years residing in Western Finland; 5,177 of whom underwent an oral glucose tolerance test (OGTT). Of them, 5,040 answered a questionnaire (Mental Health Inventory on depressive symptoms), and 4,905 participants were genotyped for the rs10830963. The Helsinki Birth Cohort Study (HBCS) with 1,576 individuals was used for replication. HBCS had identical phenotypes and participants were genotyped for the rs10830963.

Results: In the discovery cohort depressive symptoms were associated with fasting insulin and $30 \mathrm{~min}$ glucose during the OGTT, Homeostasis-Model-Assessment-Insulin-Resistance, Insulin-Sensitivity-Index and Disposition-Index in a genotype-dependent manner in insulin-resistant individuals. The same associations remained significant in insulin-resistant individuals who got tested during the dark season. In the replication cohort depressive symptoms were not associated with glycemic traits in a genotype-dependent manner in insulin-resistant individuals. However, depressive symptoms were associated with Insulin-Sensitivity-Index and AUC and $120 \mathrm{~min}$ insulin in a genotype-dependent manner in insulin-resistant individuals who got tested during the dark season.

Conclusions: Our findings support the view that the melatonergic system contributes to the known comorbidity between depression and T2D.

\section{GWAS of educational attainment, phase 3: biological findings}

James Lee, University of Minnesota, Twin Cities, United States; Aysu Okbay, Free University, Amsterdam, Netherlands; Robbee Wedow, University of Colorado Boulder, United States; Edward Kong, Harvard University, United States; Patrick Turley, Broad Institute of MIT and Harvard, United States; Meghan Zacher, Harvard University, United States; Kevin Thom, New York University, United States; Anh Tuan Nguyen Viet, University of Southern California, United States; Omeed Maghzian, Harvard University, NBER, United States; Richard Karlsson Linnér, Vrije Universiteit Amsterdam, Netherlands; Matthew Robinson, The University of Queensland, Australia; Social Science Genetic Association Consortium, United States; Peter Visscher, The University of Queensland, Australia; Daniel Benjamin, University of Southern California, United States; David Cesarini, New York University, United States

Genetic factors are estimated to account for at least $20 \%$ of the variation across individuals for educational attainment (Rietveld et al., 2013). The results of the latest GWAS for educational attainment identified 74 genome-wide significant loci for educational attainment (Okbay et al., 2016). Here, in one of the largest GWAS to date, we increase our sample to nearly 750,000 individuals, and we identify over 600 genome-wide significant loci associated with the number of years of schooling completed. Note that at the time of presentation, we will likely have updated our meta-analysis to include over 1,000,000 individuals. In this presentation, I will focus on the biological implications of the GWAS results. At the time of writing, 1,656 genes are significantly prioritized, a more than 10 -fold increase since our previous report (Okbay et al., 2016). The newly significant results reinforce the biological theme of prenatal brain development and also bring to the foreground new themes that shed light on the biological underpinnings of cognitive performance and other traits affecting educational attainment. 
An investigation of the genetic and environmental risk factors for tobacco use behaviors in young adulthood

Elizabeth Do, Virginia Commonwealth University, United States; Roseann Peterson, Virginia Institute for Psychiatric and Behavioral Genetics at Virginia Commonwealth University, United States; Bradley Webb, Virginia Institute for Psychiatric and Behavioral Genetics, United States; Danielle Dick, Virginia Commonwealth University, United States; Kenneth Kendler, Virginia Commonwealth University, United States; Hermine Maes, Virginia Commonwealth University, United States

Tobacco use remains the leading preventable cause of morbidity and mortality in both the United States and worldwide. Twin and family studies have demonstrated that both genetic and environmental factors are important contributors to tobacco use; yet, few studies have examined the transition from adolescent to young adulthood-the time when many individuals are experimenting with and developing patterns of tobacco use. Thus, to address this issue, the present study investigates the role of genes and one aspect of the environment- the parental environment within a large sample of undergraduate students attending a diverse, urban university in the Mid-Atlantic region in the United States. Preliminary results demonstrate that aggregate SNP heritability significantly accounts for the variance in ever tobacco use, age of initiation, and regular use within those of European ancestry using genome-wide complex trait analyses. While conducting genome wide association studies, few significant SNPs were found to be associated with current use and cigarettes per day. Polygenic risk scores derived from top SNPs from the Tobacco and Genetics Consortium data for ever versus never regular smoker were also found to be predictive of tobacco use behaviors. Where polygenic risk scores were predictive, the main and interaction effects of the polygenic risk score and measures of parental involvement were estimated. Continued examination of the genetic and environmental architecture is necessary for the impacts it may have on public health efforts among this understudied population.

\section{DNA methylation in Alzheimer's disease associated genes}

Ida Karlsson, Karolinska Institutet, Sweden; Alexander Ploner, Karolinska Institute, Sweden; Yunzhang Wang, Karolinska Institute, Sweden; Nancy L. Pedersen, Karolinska Institutet, Sweden; Sara Hägg, Karolinska Institutet, Sweden

A previous study identified five of the genes associated with Alzheimer's disease (AD) as differentially methylated in post-mortem brain samples from AD patients compared to controls (1). We sought to investigate whether the same associations could be found in leukocytes collected pre-mortem in dementia discordant twin pairs.

We measured DNA methylation levels in leukocytes from 427 twins using the Illumina $450 \mathrm{~K}$ array, and selected $\mathrm{CpG}$ sites within SORL1 ( $n=51)$, ABCA7 $(n=215)$, HLA-DRB5 $(n=14)$, SLC24A4 ( $=53)$, and BIN1 $(n=68)$. Forty twin pairs discordant for dementia and 33 twin pairs discordant for AD were identified. Conditional logistic regression was used to compare differences in methylation levels at each $\mathrm{CpG}$ site between discordant pairs. In accordance with the previous study, the resulting p-values for individual CpGs in each locus were combined into a test statistic for the gene-wide significance using the Fisher product method. The statistical significance was assessed by random permutations, and hence a p-value for each gene was obtained.

We found a significant difference in methylation between dementia discordant pairs in SORL1 (sortilin related receptor 1, $\mathrm{p}$
$=.01$ ), and between $\mathrm{AD}$ discordant pairs in BIN1 (bridging integrator $1, \mathrm{p}=.04)$. There was no difference in methylation levels in ABCA7, HLA-DRB5, or SLC24A4 between the discordant twins. In conclusion, SORL1 and BIN1 are not only differentially methylated in cortical cells from AD patients compared to controls, but also in leukocytes collected pre-mortem from dementia discordant twin pairs. The importance of allelic variation as well as methylation variation, both in the CNS and the periphery, in dementia suggests that SORL1 and BIN1 are important in the disease process.

\section{References}

1. Yu L, Chibnik LB, Srivastava GP, et al. Association of Brain DNA Methylation in SORL1, ABCA7, HLA-DRB5, SLC24A4, and BIN1 With Pathological Diagnosis of Alzheimer Disease. JAMA neurology 2014.

\section{Multivariate analyses and genomic imaging of the well- being spectrum}

Bart Baselmans, Vrije Universiteit Amsterdam, Netherlands; Rick Jansen, VU University Medical Centre, Netherlands; Hill Fung Ip, VU, Netherlands; Jenny van Dongen, VU, Amsterdam, Netherlands; Yanchun Bao, University of Essex, United Kingdom; Melissa Smart, University of Essex, United Kingdom; Meena Kumari, University of Essex, United Kingdom; Abdel Abdellaoui, Vrije Universiteit Amsterdam, Netherlands; Margot van de Weijer, Vrije Universiteit Amsterdam, Netherlands; Gonneke Willemsen, Vrije Universiteit Amsterdam, Netherlands; Jouke Hottenga, Vrije Universiteit Amsterdam, Netherlands; Eco De Geus, Vrije Universiteit Amsterdam, Netherlands; Dorret Boomsma, Vrije Universiteit Amsterdam, Netherlands; Michel Nivard, Vrije Universiteit Amsterdam, Netherlands; Meike Bartels, Vrije Universiteit Amsterdam, Netherlands

Several phenotypes related to well-being (e.g., life satisfaction, positive affect, neuroticism, and depressive symptoms), are genetically highly correlated $\left(\left|\mathrm{r}_{\mathrm{g}}\right|>\right.$.75) suggesting one underlying genetic liability between these traits. We performed two multivariate genomewide meta-analyses (multivariate GWAMA's) of these four phenotypes to increase the power to identify associated genetic variants. The dependence between effect sizes induced by sample overlap, was estimated from GWAS summary statistics obtained from univariate GWAMA using LD score regression. Both multivariate GWAMAs aggregated the effect of a single SNP across multiple traits based on prior knowledge of the genetic correlation between these traits. In a similar fashion, we proceeded to aggregate the effect across multiple SNPs based on prior knowledge that these SNPs collectively influence the expression level of a gene transcript or the methylation level at a $\mathrm{CpG}$ site. Biological annotation revealed significant enrichment of SNP effects in regions of the genome characterized by active histone mark in several cortical and subcortical brain tissues. In order to accurately pinpoint brain regions in which genes relevant for wellbeing are actively expressed we computed differential brain expression. To interpret the results we mapped the results to a coordinate system common to multiple other neuroimaging techniques.

\section{Components and sources of well-being and ill-being: a twin study}

Ragnhild Bang Nes, Norwegian Institute of Public Health, Norway; Nikolai Czajkowski, University of Oslo, Norway; Espen Røysamb, 
University of Oslo Norway; Olav Vassend, University of Oslo, Norway

This paper gives a brief introduction to genetically-informative studies of well-being and presents findings from a multivariate twin study. Here we investigate the extent to which the underlying structure of key components of well-being (e.g., life satisfaction, optimism, self-efficacy, positive affect) and ill-being (i.e., anxiety and depression) is multidimensional, and whether well-being and ill-being are influenced by distinct genetic and environmental factors. Additionally, more specific life circumstances and events associated with environmental sources of well-being and ill-being, are examined. The analyses are based on data from altogether 1987 Norwegian male and female twins, born between 1945 and 1960, and surveyed in 2016. Measures include the Satisfaction With Life Scale, the Depression and Anxiety subscales of the Symptom Check List, the Life Orientation Test, the Generalized Self-Efficacy Scale, the Relationship Satisfaction Scale, and the Differential Emotion Scale, along with a measure of meaning in life. The data are analyzed using biometric twin modelling and the results and their implications will be discussed.

\section{The rank-order stability of cognitive performance across time in a longitudinal twin design}

Shandell Pahlen, University of California, Riverside, United States; Matt McGue, University of Minnesota, United States; Chandra Reynolds, University of California, Riverside, United States

The non-shared environmental contributions to cognitive stability in later life was investigated in two twin longitudinal samples (SATSA and LSADT) representing two different countries (Denmark and Sweden). The contributing twin samples were taken from the Interplay of Genes and Environment across Multiple Studies (IGEMS) consortium. A general cognitive factor score was created within in each sample and two individual cognitive test performances (Digits Span and Symbol Digit) were examined between twin pairs across the samples and waves. The initial intake average differed by about 10 years between the samples: SATSA (64) and LSADT (75). Twins' cognitive performances were compared to their co-twin to determine twin order for each wave. The twin ranking on each test was then used to find the percentage of consistency for the higher scoring twin for each wave with intake being the baseline. The dizygotic twins had higher percentages of twin order consistency across the two samples for all the tests examined, but only modestly higher for Digit Span and Digit Symbol. Additionally, the younger sample (SATSA) had higher consistency on twin order for the general cognitive score than the older twin sample (LSADT). A multivariate Cholesky was used to assess the non-shared environmental contributions and correlations for each sample. The declining non-shared correlations pattern for general cognitive ability paralleled between samples across waves, but the magnitude was different, with SATSA reporting correlations and moderate for LSADT. Similar to general cognitive ability, the non-shared environmental correlation pattern across waves for Digit Span agreed between samples. The pattern and strength for the nonshared environmental correlations were stable across waves $(\sim 0.3)$. Unlike general cognitive ability and Digit Span, the Symbol Digit non-shared environmental correlation pattern were divergent after the first wave assessed and the pattern for SATSA and LSADT continued to mirror each other across waves. The observed differential patterns across samples and cognitive tests need further investigation to elucidate the meaning of these findings on the etiology of cognitive stability in later life.

\section{APOE genotype and plasticity in cognitive training}

Anne Cecilie Sjøli Bråthen, University of Oslo, Norway, Norway; Ann-Marie Glas $\varnothing$ De Lange, University of Oslo, Norway; Beate Duus Wetteland, University of Oslo, Norway; Anders Martin Fjell, University of Oslo, Norway; Kristine Beate Walhovd, University of Oslo, Norway

Rationale: The major known genetic risk factor for sporadic Alzheimer's disease (AD) is the $\varepsilon 4$ variant of the apolipoprotein E (APOE) allele. APOE has been suggested to affect plasticity. The literature is scarce, but evidence from clinical populations suggest that $\mathcal{E} 4$-carriers may show less cognitive plasticity in response to intervention. As episodic memory decline is a hallmark of $\mathrm{AD}$, it is of interest to study whether APOE allelic variation impacts memory plasticity in a controlled experimental intervention.

Methods and analyses: In a study of effects of strategic episodic memory training in healthy adults in their 20 and $70 \mathrm{~s}$, we investigated the effects of the naturally occurring variation in APOE allelic variants on training gains (absence versus presence of $\varepsilon 4$-allele; $\mathrm{N}=$ 132,47 young and 85 older, of whom 23.4 and $23.5 \%$ E4-carrieres, respectively; $2.3 \%$ of the total sample had two $\varepsilon 4$ alleles). Memory gains were calculated through change in serial recall of word lists consisting of 100 words.

Results: Participants showed significant increase in memory after training compared to passive and active control conditions. On average, E4-carriers and non-carriers recalled 14.1 (SD 7.6) and 13.1 (SD 6.6) words pre-memory training, and 25.7 (SD 12.7) and 26.5 (SD 13.6) post-memory training. There were effects of intervention (pre, post) and age group, and an interaction of intervention and age group, with greater plasticity in younger $(\mathrm{p}<.001)$, but no effect or trend towards effect of APOE $(\mathrm{F}=.067, \mathrm{p}=.796)$, nor any interactions of $\mathrm{APOE}^{*}$ intervention or $\mathrm{APOE} *$ intervention*age group.

Conclusions: The present study indicated no significant effects of absence versus presence of an APOE E4-allele on memory plasticity in healthy adults. There were few with two APOE $\varepsilon 4$ alleles, effects of which should be subject to future investigations. Power analysis suggested that a sample of $>1600$ individuals would be required to detect the presently observed small difference in plasticity. Training gains were ample in both groups. Hence, the present results indicate that healthy carriers of an APOE $\varepsilon 4$ allele benefit from memory training in a largely comparable manner to non-carriers.

\section{Effects of somatic and mental illness on subjective wellbeing. A discordant twin analysis}

Nikolai Czajkowski, University of Oslo, Norway; Ragnhild Bang Nes, Norwegian Institute of Public Health, Norway; Olav Vassend, University of Oslo, Norway; Espen Røysamb, University of Oslo, Norway

Background: It is widely believed that illness, both somatic and mental, cause a transient and possibly lasting reduction in subjective wellbeing (SWB). Similarly, some studies indicate that becoming inflicted with a chronic disease will lead to a sustained reduction in SWB. However, in arguing for a causal impact of illness on SWB, few studies have investigated to what extent confounding factors influencing both selfreported illness and SWB may account for the observed association. Methods: Lifetime experience of illness was reported in a sample of 903 monozygotic (MZ) and 1084 dizygotic (DZ) twins in a cohort born 1945-1960. In all 41 illnesses were reported, spanning several classes, among them respiratory, circulatory, musculoskeletal, and mental. Linear mixed models predicting subjective wellbeing in pairs discordant on illness were fitted using the lmer package in $\mathrm{R}$. 
Results: Disregarding twin pairing, 38 (93\%) of the disorders were negatively associated with SWB, and this was significant at a 0.05 level for $27(66 \%)$ of the illnesses. Preliminary discordant MZ analyses revealed that while a number of associations were attenuated, and a significant negative association remained for a subset of the illnesses.

Conclusion: Discordant MZ analyses support the causal negative effect of illness on SWB for a subset of the disorders assessed. The strength of these associations, as well as common characteristics of illnesses with a causal and negative impact on SWB will be investigated and reported.

\section{A sibling comparison study of maternal smoking during pregnancy and child cognitive functioning}

Lauren Micalizzi, Brown University, United States; Kristine Marceau, Purdue University, United States; Leslie Brick, Brown University, United States; Rohan Palmer, Emory University, United States; Alexandre Todorov, Washington University, School of Medicine, United States; Andrew C. Heath, Washington University, School of Medicine, United States; Allison Evans, Brown University, United States; Valerie Knopik, Brown University, United States

Introduction: The current study examines if the links between maternal smoking during pregnancy (SDP) and child cognitive functioning withstand rigorous control for familial confounds.

Methods: 173 families were recruited based on birth record report in which mothers changed smoking behavior between two pregnancies. Assessments occurred when children were 7-16 years old. Mothers reported on their SDP (Todd et al., 2003). Parent and child inhibitory control (IC), set-shifting, verbal fluency (Delis et al., 2001) and auditory memory (AM; Wechsler, 1997; 2003) were assessed.

Statistical Approach: The sibling comparison approach involved fitting a series of hierarchical linear models. The standard models compared children whose mothers smoked (or smoked more) during pregnancy to those whose mothers did not smoke (or smoked less) for each outcome. The sibling comparison models assessed within- and between-family associations between SDP and each construct, allowing for a direct test of SDP effects.

Results: SDP was not associated with set-shifting or verbal fluency. Higher SDP severity was associated with worse IC and AM, suggesting a main effect of SDP. The AM-SDP association was robust to control for familial confounds, but the IC-SDP association was not. In the sibling comparison models, the within-family associations (child-specific SDP) were significant, suggesting potentially causal effects of SDP on IC and AM. The effect of SDP on AM, but not IC, was robust to control for genetic and environmental confounds. Approximately $75 \%$ of the variance in IC and AM was attributable to within-family differences. Thus, variability was primarily a function of differences between siblings rather than across families.

Discussion: These findings suggest that: (1) proximal environments may serve as effective targets of interventions seeking to improve children's cognitive functioning in families with nicotine dependent mothers; and (2) underscore the need for genetically-informed studies of the effects of SDP on child outcomes.

\section{The significant effects of ovarian hormones on genetic risk for binge eating during puberty in girls}

Kelly Klump, Michigan State University, United States; S. Alexandra Burt, Michigan State University, United States; Cheryl Sisk,

Michigan State University, United States
Puberty is a critical period for the genetic expression of binge eating in girls (Klump et al., in press, Ann Rev Clin Psych). Previous twin studies show dramatic increases in genetic influences on binge eating from pre-puberty $(\sim 0 \%)$ to mid-puberty and beyond $(\sim 50 \%)$ (Klump et al., in press, Int J Eat Disorders). However, little is known about the factors that drive these changes in genetic effects. Previous pilot studies in twins (Klump et al., 2010, Psych Med:40(10), 17451753) have suggested that the pubertal activation of ovarian hormones may contribute to increases in genetic influences, possibly via hormonally induced changes in gene expression. Nonetheless, large-scale twin studies confirming hormone effects on genetic risk are lacking, particularly those examining both estrogen and progesterone in developmentally diverse samples of girls. Thus, the purpose of the present study was to examine the effects of estrogen and progesterone on genetic influences for binge eating in a large sample of same-sex female twins. Participants included 1,000 female twins (ages 815 years) from the Michigan State University Twin Registry. Binge eating was assessed with the Minnesota Eating Behaviors Survey (von Ranson et al., 2005, Eat Behav:6(4), 373-392), while pubertal development was assessed with the Pubertal Development Scale (Petersen et al., 1988, J Youth Adol:17(2), 117-133). Afternoon saliva samples were assayed for estradiol and progesterone levels using enzyme immunoassays. Twin moderation models showed significant and substantial differences in genetic influences on binge eating by estradiol and progesterone levels, even after controlling for the effects of age, body mass index, and onset of menses. Overall, findings suggest that the activation of ovarian hormones may drive changes in genetic effects on binge eating during puberty. Additional studies are needed to identify mechanisms of effects, including potential hormone regulation of gene expression in key neural systems.

\section{Education, reproductive success and longevity: a Mendelian randomization study}

Rebecca Lawn, University of Bristol, United Kingdom; Abigail Fraser, University of Bristol, United Kingdom; Ian Penton-Voak, University of Bristol, United Kingdom; Marcus Munafo, University of Bristol, United Kingdom

It has been suggested that educational attainment is under negative selection. Recent genetic findings make it possible to directly test natural selection by analysing whether genetic variants robustly associated with various traits are also associated with reproductive success. We therefore investigated whether individuals with greater genetically predicted educational attainment had fewer offspring. Such a relationship is compatible with a slow life history strategy where less effort is directed towards reproduction in conjunction with increased longevity. We performed Mendelian Randomization analyses in UK Biobank to assess causal associations between educational attainment and both number of offspring $(\mathrm{N}=113,518)$ and longevity $(\mathrm{N}=44,089)$, using single nucleotide polymorphisms associated with years of education $\left(\mathrm{p}<5 \times 10^{-8}\right)$. As predicted, educational attainment was negatively associated with number of offspring (mean difference: $-0.09,95 \%$ CI: $-0.12,-0.06$ per SD increase in education score) and positively associated with longevity (mean difference: $0.07,95 \% \mathrm{CI}$ : 0.04, 0.10 per SD increase in education score). Results were similar when males and females were analysed separately. Our results suggest educational attainment is under negative selection, as individuals with greater genetically predicted educational attainment have fewer offspring. 
Are genetic and environmental influences on objective and subjective health in late middle age moderated by socioeconomic status?

Carol Franz, University of California, San Diego, United States; Asad Beck, San Diego State University, United States; Michael Lyons, Boston University, United States; Matthew Panizzon, University of California, San Diego, United States; William Kremen, University of California, San Diego, United States; IGEMS Consortium, University of Southern California, United States

Despite decades of research on the relationship between socioeconomic status (SES) and health, key questions remain unresolved. First, studies seldom examine mechanisms underlying the SES-health gradient. Second, operationalization of SES continues to be problematic. Measures of SES (i.e., education, occupation, income, and their composites) tend to be used and interpreted interchangeably in twin studies. We analyzed the extent to which childhood and adult SES moderate genetic and environmental influences on health in an attempt to better understand mechanisms by which inequality contributes to health. We also evaluated the effects of different SES measures on health outcomes. Method. Participants were $\sim 1000$ monozygotic and dizygotic male twins ages 51-60 ("age 56") and 5667 ("age 62") from the Vietnam Era Twin Study of Aging (VETSA). Objective health $(\mathrm{OH})$ was a count of 15 major chronic health conditions elicited by the question "have you ever been told by a physician that you had any of the following conditions or illnesses." Subjective health $(\mathrm{SH})$ was assessed with the question: "In general, how would you rate your health?" (excellent to poor) from the SF36v1. OH and SH were correlated 0.30. Measures of SES included own, spouse, maternal and paternal occupation and education, own income, and the Hollingshead-Redlich social status index (HR-Index) which combines occupation with education across spouses using a weighted formula. Separate childhood and adult HR-Indices were created. Results. Both $\mathrm{OH}$ and $\mathrm{SH}$ were significantly heritable and consistent over time ( $\mathrm{h} 2=0.37 ; 0.31 \mathrm{OH}_{56}, \mathrm{OH}_{62}$ respectively; h2-= $0.33,0.30 ; \mathrm{SH}_{56}, \mathrm{SH}_{62}$ respectively). Remaining variance was primarily explained by unique environmental influences with very small effects for common environment. There was evidence for significant new genetic and environmental influences specific to $\mathrm{OH}_{62}$ and $\mathrm{SH}_{62}$. Moderator effects varied according to SES measure and outcome. For instance, the current-but not maximum - adult HR-Index moderated means and variance for $\mathrm{SH}_{62}$. Unique environmental variance for $\mathrm{SH}_{62}$ was significantly higher for lower SES men. We discuss the impact of different SES measures, reasons for the differential effects of SES on genetic and environmental influences on health, and we consider the challenge of such characteristics as age, gender, cohort, culture and time.

\section{The relationship between avoidant personality disorder, social anxiety disorder and normative personality traits: a longitudinal twin study}

Audun Welander-Vatn, Norwegian Institute of Public Health, Norway; Fartein Ask Torvik, Norwegian Institute of Public Health, Norway; Nikolai Czajkowski, University of Oslo, Norway; Kenneth Kendler, Virginia Commonwealth University, United States; Ted Reichborn-kjennerud, Norwegian Institute of Public Health, Norway; GunPeggy Knudsen, Norwegian Institute of Public Health, Norway; Eivind Ystrom, Norwegian Institute of Public Health, Norway

Avoidant personality disorder (AvPD) and social anxiety disorder (SAD) share risk factors to a substantial degree, and both are characterized by the experience of anxiety in social situations. We investigated whether these disorders are differentially related to normative personality traits. We also examined the underlying genetic and environmental influences on these associations to identify disorder-specific effects. AvPD and SAD were assessed in a populationbased sample of 1,761 female twins at baseline, and 1,471 of these 10 years later. At the second wave of data collection we also measured the Big Five personality traits. Associations between AvPD, SAD and personality traits were investigated with multivariate biometric analyses. On the phenotypic level, AvPD was associated with lower levels of extraversion, openness to experience, and agreeableness than SAD. Genetic risk factors for AvPD, compared to SAD, had a weaker positive correlation with genetic factors underlying neuroticism, and a er negative correlation with genetic factors underlying extraversion and openness to experience. The genetic liability to AvPD could be fully accounted for by genetic factors influencing SAD and normative personality. AvPD and SAD also differed on the environmental correlations, with a higher positive correlation with neuroticism and higher negative correlations with extraversion and openness to experience identified for AvPD. Environmental factors underlying SAD and personality traits fully explained environmental risk for AvPD. AvPD and SAD are differentially related to several personality traits at the phenotypic, genetic, and environmental level, and SAD and personality traits may together account for the genetic and environmental liability to AvPD.

\section{Subjective well-being revisited: what's in a genetic correlation?}

Jennifer Harris, The Norwegian Institute of Public Health, Norway; Julia Kutschke, Medical Department, Vestfold Hospital Trust, Norway

SWB is a multidimensional construct conceptually rooted in two broad domains encompassing hedonic and eudaimonic well-being. Behavioral genetic studies provide good evidence for a common genetic factor underlying SWB and its component traits1, 2. Functional genomic studies suggest differential transcriptional profiles between some components of SWB, with healthier patterns of gene expression associated with eudaimoniasup3/sup. However, these findings have been met with criticism, partly because the high correlation between hedonic and eudaimonic well-being casts improbability on the prospect of distinct gene regulatory profiles 4 . To gain further insight into this we employ multivariate modeling to explore the genetic covariance structure across three dimensions of SWB. The sample is based on 5442 Norwegian twins, aged 40-80, who participated in a study on Social Factors and Health. Self-reports of well-being were assessed using the short form of the Mental Health Continuum (MHC-SF). The three sub-scales of the MHC-SF cover hedonic and eudaimonic dimensions and include psychological, social, and emotional well-being. A common pathway model best described the covariance structure between the sub-scales, and revealed sex differences in the variance composition with $\mathrm{C}$ effects for females only. Phenotypic correlations between the subscales ranged from 0.58 to 0.70 . Although genetic correlations were also sizeable (0.63-0.72), there are significant amounts of trait-specific genetic effects and the correlational relationships between the subscales are, largely, explained by environmental factors. The picture emerging of the underlying genetic effects across these three components of SWB indicates only partial overlap in the sets of genes affecting trait variation and does not preclude the possibility of differential transcriptional profiles. 
References

1. Bartels M, Boomsma DI. Born to be happy? The etiology of subjective well-being. Behav Genet 2009;39(6):605-15.

2. Keyes CL, Myers JM, Kendler KS. The structure of the genetic and environmental influences on mental well-being. Public Health. 2010;100(12):2379-84.

3. Fredrickson BL, Grewen KM, Algoe SB, Firestine AM, Arevalo JM, Ma J, Cole SW. Psychological well-being and the human conserved transcriptional response to adversity. PLoS One. 2015;10(3): e0121839.

4. Coyne JC. Highly correlated hedonic and eudaimonic wellbeing thwart genomic analysis. Proc Natl Acad Sci US A. 2013;110 (45):E4183.

\section{Using gene set enrichment analysis to create polygenic risk scores for childhood externalizing behavior}

Kit Elam, Arizona State University, United States; Charles Beekman, Arizona State University, United States; Thomas Dishion, Arizona State University, United States; Daniel Shaw, University of Pittsburgh, United States; Melvin Wilson, University of Virginia, United States; Kathryn Lemery-Chalfant, Arizona State University, United States

GWAS-based polygenic risk scores (PRS) for childhood externalizing behavior have predicted aggression, impulsiveness, and conscientiousness (Salvatore et al., 2015). Selection of SNPs for PRS is typically statistically-driven, irrelevant of biological mechanisms. We used gene set enrichment analyses (GSEA) to inform selection of SNPs from a discovery GWAS to create PRS from biologically-related genes, and tested associations with childhood externalizing behavior. Results from a Meta-GWAS of childhood externalizing behavior (Pappa et al., 2016) were mapped to gene sets containing 10-200 genes using iGSEA4GWASv2 (Zhang et al., 2015). Using SNPs at gene-set significance levels of FDR $\mathrm{p}<.01, \mathrm{p}<.05, \mathrm{p}<.10$, and $\mathrm{p}<.20$ (136-252 SNPs), PRS were created. PRS were also created for SNPs from the original GWAS at the same significance levels (1570-29,433 SNPs). Participants were from a longitudinal randomized prevention trial of high-risk children. Children in the current subsample were $2-5$ years old $(n=391), 48 \%$ female and 55\% Caucasian, 29\% African American, 13\% Hispanic, 3\% Other Parents reported on the externalizing subscale of Achenbach's CBCL in each year. A latent measure of aggression was created across ages 2-5 (RMSEA=.04, CFI=.99). Analyses controlled for child age, ethnicity, gender, and intervention condition. PRS created from the original GWAS were individually tested at each significance level and none were associated with childhood externalizing behavior $\left(\mathrm{p}>.16, \mathrm{R}^{2}\right.$ $=.001-.009$ ). GSEA-scores at $\mathrm{p}<.05, \mathrm{p}<.10$, and $\mathrm{p}<.20$ significance levels were significantly associated with childhood externalizing behavior ( $\beta=.11^{*}, \beta=.13^{*}, \beta=.12^{*}$, respectively), each explaining $1-$ $2 \%$ of variation in behavior. Additional analyses will be presented on prediction of trajectories of externalizing behavior. Although these preliminary results require replication, using GSEA-informed polygenic risk explained more variation in child externalizing behavior than statistically-driven polygenic risk. GSEA is a promising method for advancing understanding of genetic association with quantitative traits.

\section{Early family adversity predicts peer outcomes in middle childhood: a genetically-informed twin study}

Gianna Rea-Sandin, Arizona State University, United States; Sierra Clifford, Arizona State University, United States; Leah D. Doane,
Arizona State University, United States; Candace R. Lewis, Arizona State University, United States; Kathryn Lemery-Chalfant, Arizona State University, United States

Children acquire new social skills and validate their own self-concept within peer relationships (Boivin, Vitaro, \& Poulin, 2005). Although few genetically informative studies have examined peer relationships, we hypothesize both genes and the shared environment contribute to peer outcomes. Furthermore, early adversity is known to influence later behavior, therefore we hypothesize that early family stress during toddlerhood would increase victimization and decrease peer acceptance and prosocial behaviors in middle childhood. The sample included 194 twin pairs from the Arizona Twin Project (LemeryChalfant et al., 2013). Early Adversity is the first principal component of Parenting Daily Hassles, low perceived MOS social support, punitive punishment (Parental Responses to Child Misbehavior), home chaos (Confusion, Hubbub, and Order Scale), CES-D maternal depression, and low maternal emotional availability. Early adversity was assessed when the twins were 30-months and parents reported on Health and Behavior Questionnaire peer relationships when the twins were 8 years old. Mixed model regression analyses indicated that early adversity predicted more peer victimization, $\mathrm{t}(93.64)=4.045$, $\mathrm{p}<.001$, less peer acceptance, $\mathrm{t}(94.275)=-3.154, \mathrm{p}=.002$, and fewer prosocial behaviors, $\mathrm{t}(99.211)=-3.797, \mathrm{p}<.001$. The CE model fit best for victimization (shared $68 \%$ and nonshared $32 \%$ environment), and peer acceptance (51\% shared, and $49 \%$ nonshared environment). In contrast, prosocial behaviors had a large genetic component (49\%), as well as shared environment (38\%). Peers play an important role in shaping child development, especially because children in most cultures spend the majority of their day around age mates at school. Our results highlight the importance of early childhood environments and elucidate the contributions of the shared and nonshared environment to peer relationships in middle childhood. Future analyses include testing whether early adversity moderates ACE influences on peer outcomes. R01HD079520 R01HD086085.

\section{Genetic and environmental influences on cortisol response to trier social stress test}

Chelsea Sawyers, Virginia Commonwealth University, United States; Christina Sheerin, Virginia Commonwealth University, United States; Meridith Eastman, Virginia Commonwealth University, United States; Jason Burchett, Virginia Commonwealth University, United States; Ananda Amstadter, Virginia Commonwealth University, United States; Roxann Roberson-Nay, Virginia Commonwealth University, United States

Background: Cortisol is a hormone released by the hypothalamicpituitary-adrenal axis (HPA axis) and is involved in sustained response to stressful stimuli as well as maintaining several other body processes. Salivary cortisol is implicated in physiological reactions to psychosocial stress and is an indicator of HPA dysregulation. In this study, we sought to estimate genetic and environmental influences on salivary cortisol response to a psychosocial stressor.

Methods: Participants include 112 monozygotic and 158 dizygotic Caucasian adolescent and young adult twin pairs (Mean age $=16.8$, $\mathrm{SD}=1.3 ; 55 \%$ female) who participated in a Trier Social Stress Test, a well validated cortisol provocation task. Salivary cortisol samples were collected at four time points after completion of the task: immediately after concluding the task, and every 15 min following. Area under the curve (AUC) was calculated for each twin; this approach is based on repeated salivary cortisol measurements to generate a response profile that captures the overall cortisol response and rate of change over time. A univariate biometrical model was 
used to estimate additive genetic, common (shared) environment, and unique (non-shared) environmental contributions to cortisol response to psychosocial stress.

Results: MZ correlations for salivary cortisol were significant, $\mathrm{r}=.49$, and DZ twins were also significantly correlated at $r=.17$. AUC cortisol response was moderately heritable $(\mathrm{h} 2=0.47)$ with remaining variance accounted for by unique, non-shared environment. No significant influence of the shared environment was observed.

Conclusions: Cortisol response to a psychosocial stressor was moderately heritable. This study is the first to examine the heritability of AUC salivary cortisol response to a psychosocial stressor in an adolescent and young adult sample. Results suggest moderate genetic influences in adolescents and young adults. Future studies should seek to determine the stability of heritability as a function of aging and exposure to environmental stressors.

\section{Seven years of spit: an update on the spit for science project}

Danielle Dick, Virginia Commonwealth University, United States

Seven years ago, we launched a university-wide research study aimed at understanding genetic and environmental influences on substance use and mental health outcomes among college students. With nearly $70 \%$ participation rates, and almost 10,000 students enrolled, we have created a registry of emerging adults followed longitudinally across their college years and beyond. There are now more than 60 faculty from 18 different departments across the campus working with the data, and spin-off projects on selected subjects that range from imaging studies to prevention trials. This talk will provide an update on the project and new findings coming out of it, and discuss the translational partnerships that are building bridges from basic science to prevention/intervention.

\section{Genes, IQ and social status}

Gregory Carey, University of Colorado Boulder, United States

Herrnstein's (1971) syllogism concludes that "social standing ... will be based to some extent on inherited differences [in mental abilities]." Later, Herrnstin and Murray (1994, p. 25) wrote that "intelligence now pulls the train [of social class]." Here, using a phenotypic causality model on data from the National Longitudinal Study of Youth, I demonstrate that the relationship between genes, IQ and social status resembles a "relay race model" in which successive variables pass the baton to other causal variables. Specifically, the effect of IQ on social status is mediated by scholastic achievement and scholastic achievement's effect is mediated by attained education. Hence, social stratification depends as much on residual genetic effects on school grades and attained education as it does on IQ.

\section{Alcohol dependence moderates the genetic influences underlying neuroticism}

Ian Gizer, University of Missouri, United States; Jacqueline Otto, University of Missouri, United States; Christopher Bizon, University of North Carolina, Chapel Hill, United States; Kirk Wilhelmsen, University of North Carolina, Chapel Hill, United States

Aspects of personality have been widely discussed as putative endophenotypes for psychiatric disorders, including alcohol and other substance use disorders. A recent meta-analysis provided an average heritability estimate of 0.40 across personality traits, and multivariate twin studies have suggested that traits, such as neuroticism, can explain a portion of the shared etiology between many psychiatric disorders, supporting their use as endophenotypes. Nonetheless, there is also evidence suggesting that heavy alcohol consumption and other substance use leads to substantial changes in personality, which could reduce the utility of personality traits as endophenotypes. To evaluate this possible limitation, the present study used GWAS summary data from the Genetics of Personality Consortium analysis of neuroticism to create polygenic risk scores (PRSs) for this trait in an independent sample and evaluate whether the ability of the PRS to predict neuroticism was moderated by the presence of DSM-IV Alcohol Dependence. The independent sample consisted of 590 participants from the UCSF Family Alcoholism Study with available genotype and phenotype data. Genotype data were obtained from low-coverage whole genome sequencing. Alcohol dependence was assessed using the Semi-Structured Interview for the Genetics of Alcoholism (SSAGA), and neuroticism was assessed using the Big Five Inventory. Data-analysis was conducted using a linear mixed model approach with ancestry estimates for the four major continental populations included as covariates to account for population and family structure. Across a range of significance thresholds used to determine variant inclusion $(\mathrm{p}<.50-<0.01)$, the neuroticism PRS significantly predicted neuroticism in the UCSF sample. The presence of alcohol dependence was also positively related to neuroticism (pvalues $=0.03-5 e-08$ ). Notably, these main effects were qualified by a significant interaction such that the relation between the neuroticism PRS and neuroticism scores was reduced among UCSF participants diagnosed with Alcohol Dependence relative to those who were not ( $p$-values $=0.02-0.001)$. These results suggest that chronic, heavy alcohol consumption may act as an environmental exposure that increases levels of neuroticism and potentially reduces the genetic contributions to this personality trait. If confirmed, this result suggests a potential limitation to the use of personality traits as endophenotypes for psychiatric disorders.

\section{Gene $\times$ environment interplay on indicators of social inequality}

Elisabeth Hahn, Saarland University, Germany

One of the most challenging tasks in social research is the identification of determinants of individual success and failure over the life course. Research from the past 50 years has come to the conclusion that individual life trajectories arise out of a complex interplay of genes and environments. Disentangling this gene $x$ environment interplay with respect to indicators of social inequality is a central goal of psychological and sociological research. This symposium brings together recent findings on the development of different indicators of social inequalities across adolescence and adulthood within and between societies to gain a deeper understanding of how environmental factors are intertwined with genetics shaping individual life courses. First, Juliana Gottschling will present a study on the heritability of cognitive abilities using a nuclear-twin family design to assess the presence of assortative mating and cultural transmission in a representative sample of twin families from Germany. Further, given that the Scarr-Rowe hypothesis of Gene $\times$ Socioeconomic Status (SES) interaction is a core hypothesis in the nature-nurture debate, Eric Turkheimer will present and discuss recent findings on Gene $\times$ SES interactions for cognitive abilities using different methodological approaches. Third, analyses on the development of self-esteem as a key construct for a variety of positive life outcomes will be outlined by Rainer Riemann by focusing on the interplay of 
social and genetic influences on self-esteem using a moderated extended twin family design. Wendy Johnson will present a study on gene $\times$ SES interaction effects for body mass index across adolescence and adulthood comparing Germany and the US. Elisabeth Hahn will present a study on the role of cultural conditions in Germany and the US for the relative importance of genetic influences on alcohol misuse across adolescence and early adulthood. Finally, Chris Dawes will show results of a moderation model on the relationship between the polygenic score for educational attainment and voter turnout by exogenous electoral environment.

\section{Genetically informed investigation of the association between childhood adverse experiences and adult psychopathology}

Patrizia Pezzoli, Abo Akademi University, Finland; Jan Antfolk Abo, Akademi University, Finland; Alexander Hatoum, Institute for Behavioral Genetics, United States; Pekka Santtila, Abo Akademi University, Finland

The abundant literature on the developmental consequences of adverse childhood experiences, particularly on psychopathology, remains inconclusive. Here, we test the relationship between the latent dimensions accounting for the comorbidity across childhood adversities, as well as across psychopathological symptoms. Previous findings support a bifactor latent structure of psychopathology in our sample, comprising internalizing, externalizing and body-related factors, in addition to a general "P" dimension. To understand if adverse childhood experiences may determine a general liability to psychopathology, the existence of a latent dimension of childhood distress should also be addressed from a phenotypic and etiological standpoint. We used measures of psychopathology and retrospective accounts of experienced parenting style, childhood abuse and neglect in a sample of 13,024 Finnish twins and their siblings (8415 women and 4609 men, age range 18-49). Factor analyses suggested the presence of four latent sources of co-variation between childhood adversities: emotional neglect, physical abuse, belittling and insecurity. A biometric model indicated small genetic contribution, moderate shared and moderate-to-high unique environmental influences on these factors. Employing a common pathway model, we plan to test whether the "childhood distress" and the "P" dimensions mediate the genetic and environmental influences on their first-order factors. With direction of causation modeling, we will examine the strength, nature and direction of the association between these dimensions.

\section{Relations among parental self-efficacy, emotional availability, and children's negative emotionality: a genetically-informed, longitudinal twin study}

Veronica Oro, Arizona State University, United States; Leah Doane, Arizona State University, United States; Kathryn Lemery-Chalfant, Arizona State University, United States

\section{R01HD079520.}

\section{R01HD086085.}

Evidence demonstrates associations between parental self-efficacy and child negative emotionality (Coleman \& Karraker, 2000), though research is needed to elucidate mechanisms. Parental emotional availability is a viable candidate as a mediator. It is a relational construct which captures parenting, like sensitivity, while also considering the contribution of the child (Biringen et al., 2000). We hypothesized that the association between self-efficacy and children's negative emotionality would be mediated by emotional availability. Additionally, we hypothesized that emotional availability would demonstrate shared environmental influence and emotionality would demonstrate both genetic and shared environmental influences.br.

The sample included 222 twin pairs from the Arizona Twin Project (Lemery-Chalfant et al., 2013). Parent reports of self-efficacy (Parental Cognitions Toward the Infant Scale), emotional availability (Emotional Availability Scales) and negative emotionality (Child Behavior Questionnaire) were acquired at 12-, 30-months, and 5-years.

A multilevel model accounting for twin dependence was tested to determine whether emotional availability at 30 months mediated the relation between self-efficacy at 12 months and negative emotionality at 5 years. Results indicated that self-efficacy was negatively related to negative emotionality $(\beta=-.31(.10), \mathrm{p}=.002)$ and positively related to emotional availability $(\beta=.29(.04), \mathrm{p}<.001)$. Emotional availability, in turn, was negatively related to negative emotionality ( $\beta=-1.09$ (.28), $p<.001)$. Accounting for this relation rendered the association between self-efficacy and negative emotionality nonsignificant $(\beta=-.25(.19), p=.180)$, indicating significant mediation. Estimates of the genetic, shared-, and nonshared-environmental influence for emotional availability were $.07, .88$, and .05 , and for negative emotionality were $.52, .31$, and .16 , respectively.

This keener understanding of the relations among parental selfefficacy, emotional availability, and children's negative emotionality and the genetic and environmental influences which underlie them stands to elucidate contributing factors to the parenting children receive and the outcomes they demonstrate.

\section{DUF1220 copy number and the evolutionary expansion of primate cerebral cortex}

Markus Sneve, University of Oslo, Norway; Anders Martin Fjell, University of Oslo, Norway; Kristine Beate, Walhovd University of Oslo, Norway

When compared to brains of other primates, the most striking feature of the human brain is its exceptional size. This size difference is mainly due to a massively expanded cerebral cortex and, thus, a higher number of brain neurons. The growth of the primate cortex has however not been uniform, with a set of key regions in lateral temporal, parietal and prefrontal association cortex showing disproportionally high expansion. Extreme gene duplication has been suggested as a possible evolutionary mechanism underlying the rapid cortical expansion seen in the primate lineage. The NBPF gene family has received particular interest as it contains sequences encoding DUF1220, a protein domain (of unknown function) showing more human lineage specific copy number increases than any other protein encoding sequence in the human genome. DUF1220 copy number has been linked to differences in brain size and neuron number across primates. Furthermore, variations in DUF1220 copy number across humans has been used to predict head circumference, and associations with micro/macrocephaly have been reported. Taking advantage of gene expression maps made available in the Allen Human Brain Atlas (AHBA), we tested whether variations in NBPF expression (i.e., DUF1220 dosage) across the cortex was associated with the evolutionary expansion of a given cortical region. Interestingly, in the AHBA sample of 6 adult brains we found low levels of NBPF expression in evolutionary high-expanding parts of cortex and high expression in low-expanding parts. This negative correlation was replicated in another gene, TBC1D3, which shares similar humanspecific copy-number increases as NBPF. Investigating an atlas of the developing human brain (BrainSpan), however, we found high levels 
of NBPF/TBC1D3 expression globally across the brain during the gestation period and early childhood, followed by reduced expression levels in late childhood and adolescence. The results suggest that DUF1220/TBC1D3 dosage may play a role in proliferation during human neurogenesis, but contribute to growth suppression in adulthood.

\section{Sex differences in cognitive restraint and body mass index: the COTASS-2 study}

Carol Kan, King's College London, United Kingdom; Moritz Herle, University College London, United Kingdom; Kaushalya Jayaweera, Institute for Research \& Development Sri Lanka, Sri Lanka; Anushka Asikari, Institute for Research \& Development Sri Lanka, Sri Lanka; Sisira Siribaddana Rajarata, University of Sri Lanka, Sri Lanka; Helena Zavos, King's College London, Institute of Psychiatry, Psychology \& Neuroscience, United Kingdom; Athula Sumathipala, Keele University, United Kingdom; Khalida Ismail, King's College London, United Kingdom; Matthew Hotopf, King's College London, United Kingdom; Janet Treasure, King's College London, United Kingdom; Fruhling Rijsdijk, King's College London, MRC Social, Genetic and Developmental Psychiatry Centre, Institute of Psychiatry, Psychology and Neuroscience, London, UK, United Kingdom

Background: Cognitive dietary restraint occurs when an individual monitors and limits their dietary intake to achieve/maintain a certain body weight. Studies have suggested a genetic component to cognitive restraint and BMI. In addition, sex differences in their aetiologies has been reported in Western populations but not in non-Western populations. We therefore aim to systematically examine this question in a Sri-Lankan population, using genetic model-fitting analysis.

Method: 3561 individuals were recruited to participate in the Colombo Twin and Singleton Study-Phase 2 (842 monozygotic, 578 dizygotic twin pairs and 2,019 singletons), measuring cognitive restraint and BMI. Structural equation modelling was conducted to estimate the genetic and environmental contribution.

Results: The phenotypic correlations between cognitive restraint and BMI were significant in both males $(0.48,95 \%$ confidence interval: $0.41-0.54)$ and females $(0.47,95 \%$ CI: $0.41-0.52)$. Genetic factors play a more significant role in the aetiology of both cognitive restraint and BMI in females (cognitive restraint $45 \%$, 95\% CI: 16-60\%; BMI: $77 \%, 95 \%$ CI: $53-81 \%$ ) than males (cognitive restraint: $24 \%, 95 \%$ CI: 2-51\%; BMI: 48\%, 95\% CI: $23-77 \%$ ).

Conclusions: Our results replicate previous findings of a genetic aetiology in cognitive restraint and BMI, with greater genetic effect being observed in females. Our study illustrates the importance of considering the influence of sex in studies of eating behaviours and BMI. Further research is needed to clarify whether genetic factors influence cognitive restraint and BMI directly or through inherited mediators, such as impulsivity.

\section{Genetic and environmental contributions to video- referenced ratings of reciprocal social behavior in toddler twins}

Julia D. Grant, Washington University School of Medicine, United States; Natasha Marrus, Washington University School of Medicine, United States; Anne L. Glowinski, Washington University School of Medicine, United States; Arpana Agrawal, Washington University in St. Louis, United States; Brooke Harris-Olenak, Palo Alto Veterans Affairs Health Care System, United States; Jon Randolph Haber, Palo
Alto Veterans Affairs Health Care System, United States; Andrew C. Heath, Washington University School of Medicine, United States; John N. Constantino, Washington University School of Medicine, United States

Reciprocal social behavior (RSB; the capacity to engage in social behaviors with others) is a primary deficit among individuals with autism spectrum disorders (ASDs). The video-referenced Rating of Reciprocal Social Behavior (vrRSB) was designed to quantify variability in RSB among toddlers at an age prior to most ASD diagnoses by having caregivers watch a 3-minute video of a typically-developing 19-month-old interacting with an adult and then rate their own child's RSB in comparison to the videotaped scoring anchor. Exploratory factor analysis (EFA) of the 13-item vrRSB with a community-based singleton sample $(n=1563, M=19.3$ months at caregiver rating) yielded a two-factor solution with boys and girls combined (preliminary analyses indicated comparable factors across sex). Factor 1 was interpreted as "affective engagement" (Eigenvalue $=5.73$ ) and Factor 2 as "functional communication skills" (Eigenvalue $=1.24$ ), with an inter-factor correlation of 0.52 . We applied this factor structure to confirmatory factor analysis (CFA) with a generalpopulation toddler twin sample (both members of $\mathrm{MZF}=63, \mathrm{MZM}=$ $63, \mathrm{DZF}=51, \mathrm{DZM}=50$, and $\mathrm{DZO}=90$ pairs; 2 individuals without cotwin rating; $M=18.5$ months at caregiver rating). We extracted individual factor-scores from the CFA, and examined genetic and environmental contributions to variance in and covariance between these latent constructs. Bivariate Cholesky analyses indicated significant $\mathrm{A}$ on both factors for girls $(\mathrm{a} 2=0.45-0.51)$ and boys $(\mathrm{a} 2=$ $0.81-0.88$ ), but significant $\mathrm{C}$ only for girls (c2=0.35-0.37). For girls, covariation between factor scores was attributable to $\mathrm{A}, \mathrm{C}$, and individual-specific factors $\left(E ; r_{A}=0.81, r_{C}=1.00, r_{E}=0.52\right)$. For boys, covariation was attributable to both $\mathrm{A}$ and $\mathrm{E}\left(\mathrm{r}_{\mathrm{A}}=0.89, \mathrm{r}_{\mathrm{E}}=\right.$ 0.73 ). Given that the vrRSB can capture heritable variability in RSB among toddlers at an early stage of verbal development, the vrRSB could provide an important tool for the assessment of response to treatment in ASD-focused early-interventions.

\section{Polygenic risk scores for alcohol dependence predict subjective response to alcohol in a community sample of regular drinkers}

Jacqueline Otto, University of Missouri, United States; Ian Gizer, University of Missouri, United States; Joseph Deak, University of Missouri, United States; Bruce Bartholow, University of Missouri, United States; Xianyong Yin, University of North Carolina, Chapel Hill, United States; Kirk Wilhelmsen, University of North Carolina, Chapel Hill, United States

Alcohol dependence (AD) is a moderately heritable trait with a highly polygenic genetic architecture. Modeling the cumulative effects of nominally associated variants using polygenic risk scores (PRSs) has been shown to increase the proportion of variance accounted for in substance use phenotypes, but has been largely restricted to the prediction of diagnostic status or quantitative consumption measures. As such, studies of alcohol use and dependence have not focused on intermediate phenotypes underlying risk for these traits, such as a person's subjective response to alcohol. Therefore, the current study sought to evaluate the ability of a PRS for AD to explain variation in measures of alcohol sensitivity. Summary statistics from a large-scale genome-wide meta-analysis of $\mathrm{AD}$ diagnosis were used to compute PRSs across eight significance thresholds. These scores were used to predict measures of subjective response to alcohol in a communitybased sample of regular drinkers $(n=426)$. Phenotypes included retrospective measures of subjective response to alcohol, and self-reported 
subjective intoxication and alcohol-related stimulation and sedation following administration of a moderate dose of alcohol during an alcohol challenge session. PRSs for AD were associated $(\mathrm{p}<.05)$ with variation in alcohol sensitivity during the alcohol challenge, including higher levels of alcohol-related stimulation and subjective intoxication across ascending and descending limbs of the blood alcohol concentration (BAC) curve, and lower levels of alcohol-related sedation on the descending limb of the blood alcohol concentration curve. This pattern of effects was consistent with the significant associations of the PRSs with retrospective measures of subjective response to the stimulating and sedating effects of alcohol. These results provide preliminary support that cumulative measures of genetic risk for alcohol dependence may be related to measures of subjective response to alcohol, such as a dampened response to sedating effects and increased sensitivity to the simulating effects of alcohol.

\section{Reconstructing personality from an etiologic perspective}

Jaime Derringer, University of Illinois at Urbana-Champaign, United States

Psychometric evaluation of outcomes, even in genetically informed samples, typically relies on patterns of overall phenotypic correlations, based on the assumption (whether implicit or explicit) that the etiologic structure will recapitulate the phenotypic structure. However, quantitative genetic models of continuous psychological constructs overwhelmingly support a generalist genes model (Kovas \& Plomin, 2006, Trends Cogn Sci; Kendler et al., 2011, Am J Psychiatry), where genetic factors account for covariance among phenotypes, and environmental factors account for specificity. Nonhuman animal models of personality suggest broad targets of biological influence, which may map more closely to the two-factor model of personality (e.g. approach versus avoidance) compared to the five-factor phenotypic model most commonly used in human research. This study developed personality phenotypes on the basis of genetic and environmental overlap, rather than the other way around. Item-level genetic and environmental correlation matrices were constructed using GCTA (Lee et al., 2012, Bioinformatics), which were then factor analyzed to independently identify the structure of heritable and non-heritable influences. The resulting genetic and environmental factor models suggest alternative conceptualizations of personality constructs may be useful in different research contexts.

\section{Genetic overlap between executive functions and body mass index in childhood}

Alexis Frazier-Wood, Baylor College of Medicine, United States; Uku Vainik, McGill University, Canada; Daniel Briley, University of Illinois at Urbana-Champaign, United States; Laura Engelhardt, University of Texas at Austin, United States; Andrew Grotzinger, University of Texas at Austin, United States; Jessica Church, University of Texas, United States; Paige Harden, University of Texas at Austin, United States; Elliot Tucker-Drob, University of Texas, United States

Executive functions (EFs) are cognitive processes foundational to complex reasoning and goal-directed behavior. EFs share genetic variance between themselves and with general cognitive ability (IQ), and predict academic achievement. Obesity is also inversely associated with academic achievement and IQ, highlighting its importance to cognitive development. Recently, an inverse relation has emerged between body mass index (BMI) and EFs; however the underlying mechanisms are not well understood. The aim of the current study was to decompose the inverse EF-BMI association into genetic and environmental influences, and to gauge the specificity of any EF-BMI association in relation to general cognitive abilities and academic achievement. Twins ages 7-15-year-old participating in the Texas Twin Project $(\mathrm{N}=896)$ completed a neuropsychological test battery measuring core EFs (inhibition, working memory, switching and updating), academic achievement (in reading and mathematics), and general cognitive abilities (IQ, crystalized intelligence, fluid intelligence and processing speed), and had their heights and weights measured. Associations were run between BMI and cognitive variables, specifying EFs as individual domains and as a higher-order common factor thought to represent the capacity to control and regulate attention selectively. BMI was inversely associated with nearly all cognitive measures (FDR-corrected P-values $[\mathrm{Qs}] /<.05$ ). However after controlling for age, sex, and race/ethnicity, only updating $(\mathrm{r}=$ -.321; $\mathrm{Q}=.04)$ and the latent $\mathrm{EF}$ factor $(\mathrm{r}=-.125 ; \mathrm{P}=.01 \mathrm{Q}=.04)$ remained significant. The inverse association between BMI and the latent EF factor was due to a significant genetic correlation accounting for $80 \%$ of the phenotypic association $\left(\mathrm{r}_{\mathrm{G}}=-.15 ; \mathrm{P}<.001\right)$. These findings confirm that the ability to self-regulate attention is reduced as BMI increases, and reveal this is primarily attributable to shared genetic influences between EF and BMI. This adds to a growing body of research suggesting there are a set of genetic variants important to both physical health and cognitive functioning.

\section{Polygenic and pleiotropic effects on clinical heterogeneity in major depression}

\section{Tim Bigdeli, SUNY Downstate, United States}

Srdjan Djurovic Oslo Norway Currently, a diagnosis of MD requires that at least five of nine DSM accessory symptoms be present, although patients vary with respect to the particular combination of symptoms endorsed. These nine criteria do not appear to reflect a single underlying genetic factor; instead, concordance among 7,500 adult twin pairs was best explained by three factors representing psychomotor/cognitive, mood, and neurovegetative dimensions of MD (Kendler et al. 2013). In particular, neurovegetative and reversed neurovegetative symptoms-reflecting depression-related changes in weight, appetite, and sleep-seem to implicate energy balance, and despite numerous associations between MD and relevant, comorbid medical conditions (e.g. obesity), there has been limited research on shared liability that addresses heterogeneity in a genetically informed framework. Using detailed clinical information and molecular genetic data from the CONVERGE study of MD in Han Chinese women, along with publicly available genome-wide summary statistics, we consider the evidence in support of widespread pleiotropy between MD and a range of anthropometric traits and metabolic outcomes, and compare the polygenic profiles of MD cases reporting contrasting neurovegetative and reversed neurovegetative symptoms. Subsequent genome-wide association studies (GWAS) employ a 'case-only' approach to identify associations between single nucleotide polymorphisms (SNPs) and symptom-based outcomes (e.g. weight gain versus weight loss). In collaboration with independent large-scale European studies, we replicate and perform trans-ancestry fine-mapping of detected loci. Finally, we discuss implications of convergent pleiotropy and comorbidity between psychiatric and metabolic outcomes for genetic studies of multifactorial traits.

The heritability for severe mental disorders is estimated to 60 $80 \%$ and they are now regarded as complex genetic disorders, which are associated with the effects of multiple genes (i.e. 'polygenic') in combination with environmental factors. This makes it difficult to 
determine an individual's risk, and to develop new treatments based on disease mechanisms. Genome-wide association studies (GWAS) have identified many trait-associated single nucleotide polymorphisms (SNPs). In psychiatric disorders only a small fraction of genetic variance has been identified despite recent large GWAS. This 'missing heritability' has been attributed to lack of proper statistical methods for analysis of the 'polygenic architecture'. A large proportion of the 'missing heritability' is available within GWAS data when associations of SNPs are examined in aggregate. This implies the existence of numerous small genetic ('polygenic') effects that cannot be detected with traditional statistical methods. Thus, there is a need for innovative statistical approaches to enhance discovery of common variants. We have recently developed 'enrichment tools', a statistical framework for analyzing GWAS data building on Bayesian methods. Using summary statistics derived from SNP associations of large GWAS, functional genic elements showed differential contribution to phenotypic variance, with some categories (e.g. regulatory elements and exons) showing enrichment for phenotypic association. Leveraging pleiotropy, i.e. genetic associations between two phenotypes from independent GWAS, the power for detecting small genetic effects is substantially increased, and novel susceptibility loci can be discovered. Applying these enrichment factors together in a new covariate modulated mixture model we identified several novel gene loci for schizophrenia. Of great interest is that many of the genes associated with the discovered loci are involved in neuronal excitability, including neurotransmitter systems (e.g., glutamatergic, dopaminergic, serotonergic, GABA-ergic, cholinergic) and ion channels $\left(\mathrm{Na}^{+}, \mathrm{K}^{+}, \mathrm{Ca}^{2+}\right.$, and $\left.\mathrm{Cl}^{-}\right)$. Thus novel Bayesian tools can increase power for uncovering more of the missing heritability (polygenic architecture) of psychiatric disorders, can increase discovery of gene loci that are more likely to replicate, and can improve risk prediction in new samples. Current work is focusing on improving these method and

\section{Overall abstract}

Ole A. Andreassen, University of Oslo/NORMENT, Norway; Srdjan Djurovic, Oslo, Norway; Ole A. Andreassen, University of Oslo/ NORMENT, Norway

Despite the fact that most psychiatric disorders are highly heritable (estimated to 60-80\%), only a small fraction of genetic variance has been identified despite recent large GWAS. This 'missing heritability' has been attributed to lack of proper statistical methods for analysis of the 'polygenic architecture'. Thus, there is a need for innovative statistical approaches to enhance discovery of common variants. We have recently developed 'enrichment tools', a statistical framework for analyzing GWAS data building on Bayesian methods. Also, by using leveraging pleiotropy, i.e. genetic associations between two phenotypes from independent GWAS, the power for detecting small genetic effects is substantially increased, and novel susceptibility loci can be discovered. Current work is focusing on improving these methods.

\section{Bivariate Gaussian mixture model for GWAS summary statistics}

Oleksandr Frei, University of Oslo/NORMENT, Norway

Bivariate Gaussian Mixture Model (GMM) is a novel statistical technique that jointly models GWAS summary statistics from two phenotypes as a mixture of causal, pleiotropic and null components. The model can be used as an enrichment test to detect presence of shared genetic architecture between the phenotypes, as well a tool to discover specific loci that simultaneously affect both phenotypes. The model estimates true proportion of causal SNPs after controlling for LD structure, provides per-SNP posterior probability of being causal together with corresponding local false discovery rate, and estimates posterior effect sizes. In this presentation we will present basic concepts behind Bivariate GMM, apply in to Schizophrenia, Bipolar Disorder and other phenotypes, and discuss future applications.

\section{A polygenic approach to evolution in human psychiatric $\&$ cognitive phenotypes}

Saurabh Srinivasan, University of Oslo, Norway; Francesco Bettella, University of Oslo, Norway, Norway

Complex human phenotypes are mainly polygenic in nature and are not likely to undergo selection in the form of major selective sweeps, but rather in subtle shifts in allele frequencies. These shifts are often hard to detect using traditional statistical methods. Our recently developed false discovery rate (FDR) framework exploits ancillary information to improve the power of genome-wide association studies (GWAS). This method can assess enrichment of polygenic effects on various phenotypes in specific genic annotation categories. We applied this method to study the effect of evolutionary metrics using data from GWASs of psychiatric and cognitive phenotypes. We used different evolutionary proxies representing various time periods of human evolution. The Neanderthal selective sweep score, which measures positive selection in humans after the human-Neanderthal split some 50,000 years ago, was used to test the hypothesis that the schizophrenia is related to the development of the complex human brain and concurrently, language skills and creative reasoning. We show that regions that underwent positive selection in humans after divergence from Neanderthals are more likely to be associated with schizophrenia. We investigated traces of schizophrenia association in less recently evolved regions like the human accelerated regions (HAR), segmental duplication regions (SD), and ohnologous regions (Ohno). These represent time periods before 200,000 years ago when hominid and chimpanzee branches split (HAR), 35-40 million years ago during the great ape-hominid split (SD), and the period of separation of vertebrate and invertebrate lineages 500 million years ago (Ohno). We could not detect significant enrichment for any of these evolutionary markers. We investigated GWAS of education attainment, college completion, and cognitive function, after the Neanderthal split. Our results indicate that SNPs in the swept regions are more likely to be associated with education attainment, college completion, and to some extent to general cognitive function. These findings help us understand the evolutionary history of mental and cognitive phenotypes. As humans evolved, their ability to think in the abstract, communicate with one another and organize in complex rule-based societies improved, but such development probably exposed them to mental pathologies like schizophrenia.

\section{Item response theory for precision medicine}

Wesley K. Thompson, Institute for Biological Psychiatry, Mental Health Services, Copenhagen, Denmark

Modern medical bioinformatics encompasses a vast number of possible markers potentially useful for diagnosis. These markers may include structured clinical interviews, self-report questionnaires, inflammatory markers, multi-modal brain imaging (both structural and functional), and whole-genome genotyping. The number of 
possible individual inputs is thus in the hundreds or many thousands, and the factorial combination of such markers is even more vast. Moreover, some markers are easy and cheap to collect, whereas others are time consuming and/or expensive. In many applications there is little guidance for clinicians on what information they should collect, when they should collect it, whether or not the added expense and effort is worth the extra information, and how to integrate all of these sources of information to provide a diagnosis, recommendation for treatment, or a prediction of outcome (prognostic judgment). We propose to address this situation by developing a theoretically sound algorithm that is robust to measurement differences, provides accurate predictions, and is intuitive to implement for clinician practitioners. Specifically, we will adapt methods of modern test theory (item response theory) to biomedical settings. The main idea is that we can consider different classes of markers as "testlets" to determine an underlying latent state (e.g., diagnostic status, responsiveness to a given treatment). As an extension to existing psychometric theory, we explicitly model dependency structure of the markers after conditioning on latent states (i.e., when local independence does not hold). We implement this method in the computation of polygenic risk scores (PRS) for schizophrenia, considering each locus to be a test item, calibrated using summary statistics from the Psychiatric Genetics Consortium 2014 meta-analysis.

\section{Leveraging characteristics of common genetic variants to improve power of gene discovery in genome-wide association study of neuroticism}

\section{Yunpeng Wang, University of Oslo, Norway}

Neuroticism reflects emotional instability, and is related to various mental and physical health issues. However, the majority of genetic variants associated with neuroticism remains unclear. Inconsistent genetic variants identified by different genome-wide association studies (GWAS) may be attributable to low statistical power. We proposed a novel framework to improve the power for gene discovery by incorporating single nucleotide polymorphism (SNP) characteristics and combining two relevant tools, relative enrichment score (RES) by Wang et al. and conditional false discovery rate (FDR) by Andreassen et al. Here, conditional FDR for each SNP was estimated given its RES based on linkage disequilibrium (LD)-weighted genic annotation scores, total LD scores, and heterozygosity. A known significant locus in chromosome $8 \mathrm{p}$ was excluded before estimating FDR due to long-range LD structure. Only one significant LD-independent SNP was detected by analyses of unconditional FDR and traditional GWAS in the discovery sample $(\mathrm{N}=59,225)$, and notably four additional SNPs by conditional FDR. Three of the five SNPs, all identified by conditional FDR, were replicated in an independent sample $(\mathrm{N}=170,911)$. These three SNPs are located in intronic regions of CADM2/, LINGO2 and EP300 which have been reported to be associated with autism, Parkinson's disease, and schizophrenia, respectively. Our approach using a combination of RES and conditional FDR can improve power of traditional GWAS for gene discovery, which provides a useful framework for the analysis of GWAS summary statistics by utilizing SNP characteristics, and helps to elucidate the links between neuroticism and complex diseases from a genetic perspective. 